\title{
A Three-Dimensional Turbine Engine Analysis Compressor Code (TEACC) for Steady-State Inlet Distortion
}

\author{
by \\ Alan A. Hale \\ Dissertation submitted to the Faculty of the \\ Virginia Polytechnic Institute and State University \\ in partial fulfillment of the requirements for the degree of \\ DOCTOR OF PHILOSOPHY \\ in \\ Mechanical Engineering
}

APPROVED:

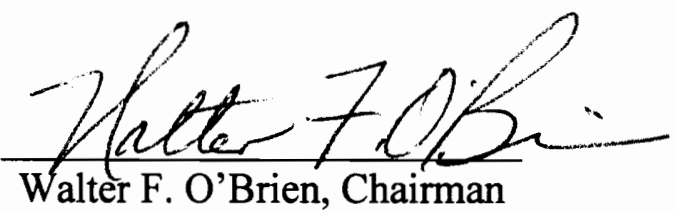

$\frac{\text { Jol Mow }}{\text { John Moore }}$
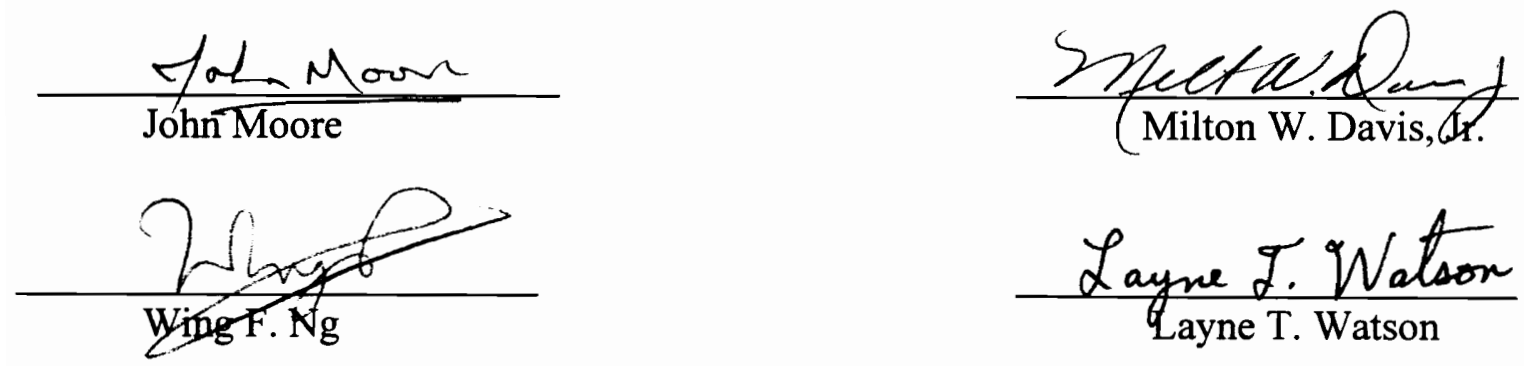

December 1996

Blacksburg, Virginia

Key words: Compressor, Three-Dimensional, Simulation, Distortion 


$$
\begin{array}{ll} 
& \text { LD } \\
C .2 & 5655 \\
& \text { V856 } \\
& 1996 \\
& \\
& 1354 \\
& c .2
\end{array}
$$




\title{
A Three-Dimensional Turbine Engine Analysis Compressor Code (TEACC) for Steady-State Inlet Distortion
}

\author{
by \\ Alan A. Hale \\ Committee Chairman: Walter F. O’Brien, \\ Department of Mechanical Engineering
}

\begin{abstract}
Modern high-performance military aircraft are subjected to rapid flight maneuvers which place great operational demands on their compression system by producing highly distorted flow to the compressor. Inlet distortion generally reduces the engine compressor stability margin and may induce compressor surge at high rotational speeds, or rotating stall at lower rotational speeds. Therefore, a computational fluid dynamics (CFD) based compressor simulation would be very useful in the design, test, and analysis process since it gives additional information with inexpensive modifications.

A new CFD simulation called the Turbine Engine Analysis Compressor Code (TEACC) was designed to meet these requirements. This code solves the compressible 3D Euler equations modified to include turbomachinery source terms which simulates the effect of the compressor blades. The source terms are calculated for each blade row by the application of a streamline curvature code. A methodology was developed for calculating turbomachinery source terms and distributing them axially, radially, and circumferentially while maintaining a sensitivity to strong inlet distortion.
\end{abstract}


TEACC was compared with experimental data from NASA Rotor 1B, a transonic rotor. Experimental data from Rotor 1B were available for comparison with TEACC results for a clean inlet and for an inlet distortion produced by a 90-degree, one-perrevolution screen. TEACC results compared very well with experimental data with a clean inlet. Comparison with experimental data with inlet distortion demonstrated TEACC's ability to characterize the compressor overall, and to accurately predict the magnitude and shape of exit total temperature and exit total pressure in the distorted region. TEACC calculated the overall character of exit total pressure and exit total temperature in the nondistorted region, identifying the location of the largest value just after the inlet distortion and the decrease in exit total values through the nondistorted region in the direction of rotation. 


\section{Dedication}

This dissertation is dedicated first to God, through Jesus Christ, who said, "Everything is possible for him who believes" (Mark 9:23) and second, to my wife, Julie Scheiman Hale, and children, Ian Bruce Hale, Vanessa Crystal Hale, and Joseph Bryan Hale. I am especially appreciative of Julie's dedicated support, which was freely given through years of sacrifice. Julie's well-spring of encouragement helped shape the dream and give additional meaning to the adventure. A special appreciation is extended to Julie, Ian, Vanessa, and Joseph for their ready acceptance to leave Tennessee and live in Virginia for one year while I completed the course work. This dissertation would not have been possible without their love. 


\section{Acknowledgments}

I am appreciative to Dr. Milt W. Davis who, in many ways, has helped shape my technical career by giving me a practical foundation to the many years of formal school training. His leadership and technical advice have ably guided me through the fundamentals of dynamic turbomachinery simulations and into multidimensional inlet distortion simulations. His belief in my abilities and willingness to support me have always been a great source of encouragement.

A special thanks goes to Mr. Karl Kneile, who helped wrestle the ARO-1 code and its many modifications into a usable duct flow simulation which was used in the initial shaping of the TEACC methodology. I appreciate Mr. Kneile's willingness to share his extensive computer expertise and numerical insight freely. But far beyond all this, I appreciate the valuable friendship that has extended through the years.

Appreciation is extended to Dr. Walter O'Brien, my major professor, who has provided technical guidance and encouragement. Dr. O'Brien has been an excellent sounding board for keeping this work on schedule.

Many people in the CFD team and modeling and simulation team have contributed through the years. A special thanks goes to Dr. Kyle Cooper and Dr. Greg Powers for their dedicated effort to develop NPARC to be extremely flexible and robust, and for their technical advise. A thanks goes to Dr. Doug Garrard and Mr. Stephen Savelle for their encouragement and support. A special appreciation is extended to Mrs. 
Jacqueline Chalk, who best understood the challenges of completing this work since she was completing a Masters degree at the same time.

I would like to acknowledge the United States Air Force and Sverdrup Technology, Inc. for granting me a leave of absence for one year so I could complete my residency requirements and course work at Virginia Polytechnic Institute. I also appreciate the opportunity to take classes and complete my research during work time.

I am eternally grateful to my parents for their diligent training to me in right behavior. Home is where I first tasted the discipline that made it possible for this work to be attempted and completed. Their unwavering support through the years has served as a beacon of encouragement to me that will always be appreciated.

I thank the body of Christ at Dayspring Christian Fellowship and Good News Christian Fellowship who prayed for me and gave me support all along the way.

A special thanks goes to Mr. Gary Owens, who has missed many rock climbing and caving trips due to my absence, and who has patiently waited for me to finish. 


\section{Table of Contents}

$\underline{\text { Section }}$

$\underline{\text { Page }}$

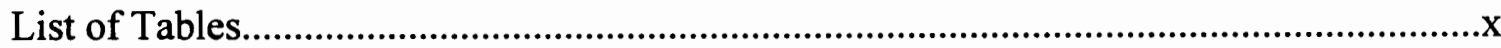

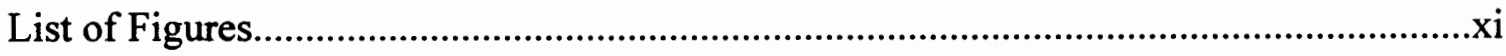

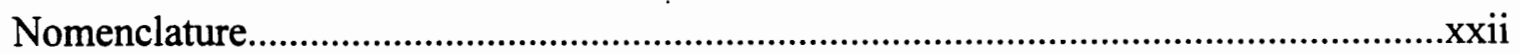

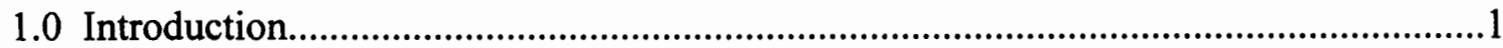

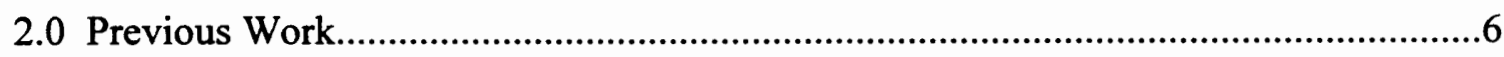

2.1 Parallel Compressor Simulation.................................................................

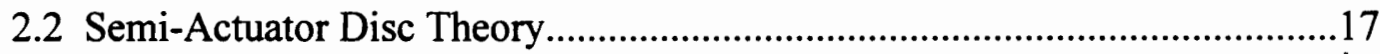

2.3 Expected Benefit of a New 3D Distortion Simulation.....................................21

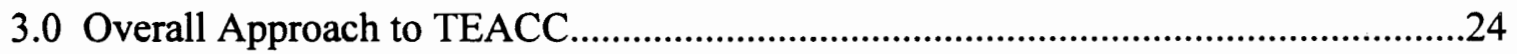

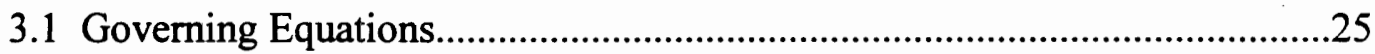

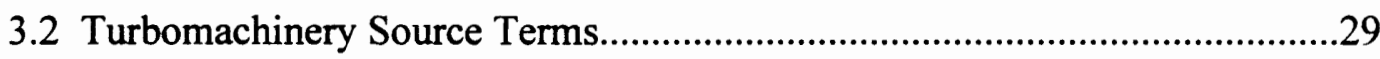

3.3 Grid Structure Tailored for Turbomachinery ..................................................39

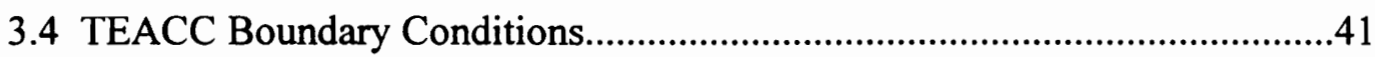

3.5 Initializing the TEACC Flow Field with SLCC............................................

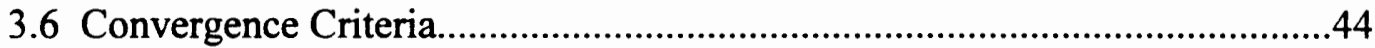

3.7 TEACC Solution Procedure 
4.0 Methodology to Implement Source Terms into TEACC..............................................48

4.1 Radial Interpolation of Turbomachinery Source Terms.................................48

4.2 Circumferential Distribution of Turbomachinery Source terms.....................49

4.3 Axial Distribution of Turbomachinery Source Terms.....................................50

4.4 Source Modifications Necessary for Stability ..............................................53

4.5 Mathematical Representation of Source Term Implementation.......................56

5.0 Experimental Data for Comparison with TEACC.....................................................58

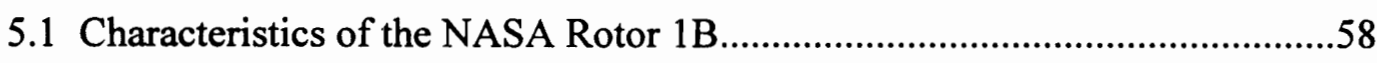

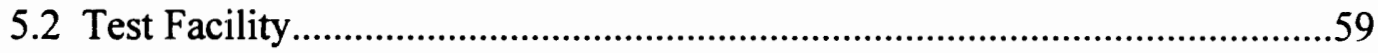

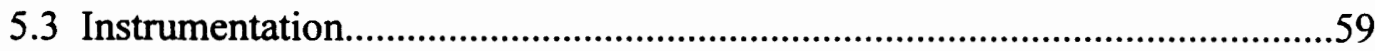

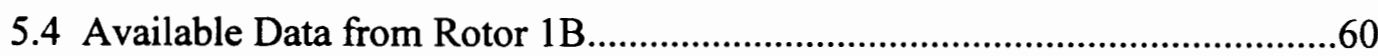

6.0 Consistency Check for Clean Inlet TEACC with Experimental Data.........................63

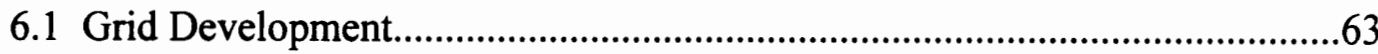

6.2 Clean Inlet Comparison of TEACC with Experimental Data..........................66

7.0 Distorted Inlet Comparison of TEACC with Experimental Data.................................72

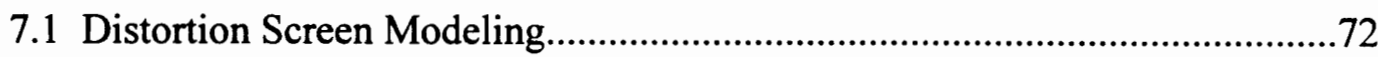

7.2 Overall Rotor 1B Performance Comparison...................................................75

7.3 Radial Comparison of Compressor with Experimental Data...........................77 


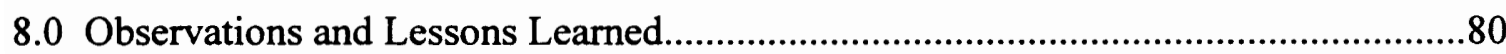

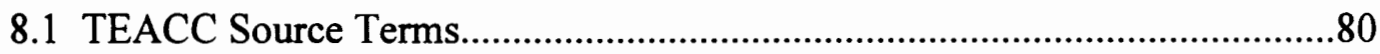

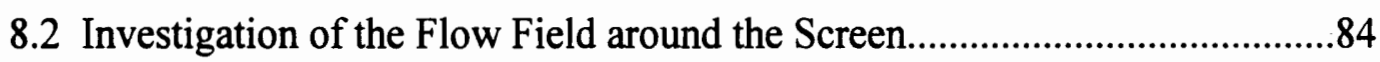

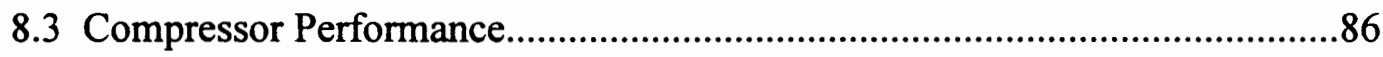

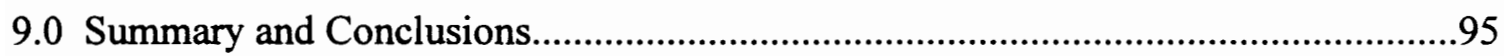

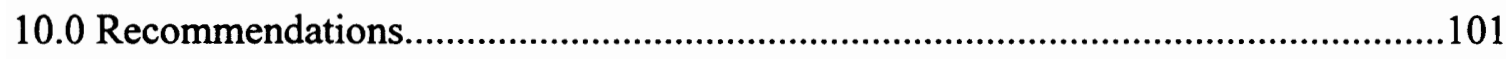

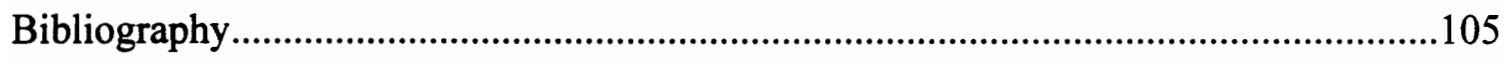

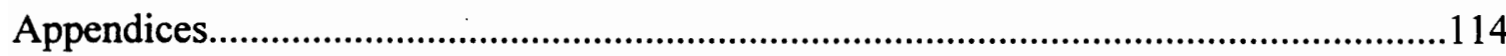

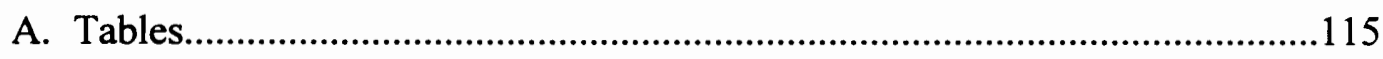

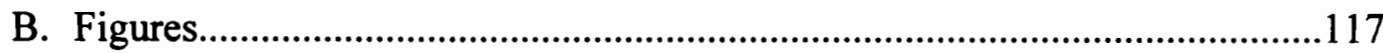

C. Review of Streamline Curvature Code Development..................................182

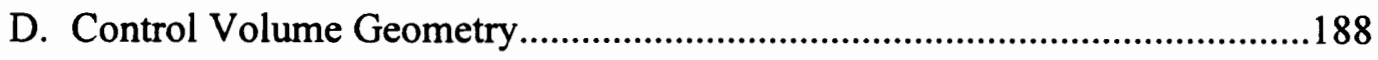

E. Calibration of the SLCC for Rotor 1B.........................................................196

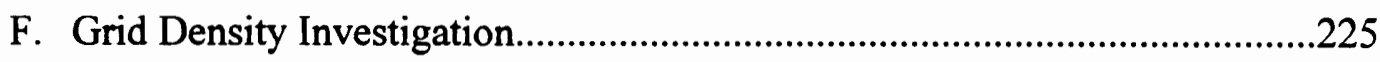

Vita 


\section{List of Tables}

Table

Page

Table 4.1: Variable relaxation factor used to relax the source term on each axial station through the bladed region by selecting an initial $\phi$ between 1.0 and $0.5 \ldots . . .115$

Table 6.1: Validation of clean inlet TEACC with experimental data .115

Table 7.1: Percent loss in total pressure across screen relative to screen inlet. .116

Table E.1: Calibrated streamline curvature code (SLCC) compared with experimental data.

Table F.1: Grid refinement investigation at $100 \%$ corrected speed near design throttle line.

Table F.2: Circumferential area relationship between cylindrical area and Cartesian area 


\section{List of Figures}

Figure

Figure 1.1: Inlet distortion defined at the aerodynamic interface plane (AIP) 118

Figure 1.2: Development of total pressure inlet distortion produced at three different flight attitudes.

Figure 1.3: Effect of 180-degree total pressure inlet distortion produced for a multi-stage high pressure compressor

Figure 1.4: Inlet distortion can lead to compressor instabilities (surge and rotating stall)

Figure 1.5: Engine screen developed to duplicate the most severe distortion patterns at the AIP

Figure 2.1: Classical parallel compressor theory with a description of how the two circumferential segments respond to total pressure inlet distortion.

Figure 2.2: Delayed lift response for rotors with circumferential inlet distortion (Korn, 1974).

Figure 2.3: Schematic of volumes for circumferential-flow redistribution model (Steenken, 1983)

Figure 2.4: Inlet flow distortion simulation results (Steenken, 1983)

Figure 2.5: Segment and particle circumferential displacement through compressor (Mazzawy, 1977).

Figure 2.6: Modified multiple-segment model for single-stage fan (Mazzawy, 1977).

Figure 2.7: Total pressure, static pressure, and axial velocity distributions at a far upstream station and at the compressor inlet (Longley, 1992).

Figure 2.8: Parallel diffuser model for compressor-component coupling effects; a) parallel diffusers, b) non-uniform flow in a parallel diffuser (Longley, 1992). 
Figure 2.9: Effect of downstream components on compressor performance with inlet distortion (Longley, 1992).

Figure 2.10: Effect of downstream components on static pressure distortion at exit of three-stage compressor (Longley, 1992).

Figure 2.11: Model-computed influence of circumferential pressure distortion on stability, XC-1 compressor (Kimzey, 1977).

Figure 2.12: Comparison of circumferential distortion model computations with experimental results (Kimzey, 1977)

Figure 2.13: Computed radial distortion influences on stability (Kimzey, 1977).

Figure 2.14: Comparison of radial distortion model computations with experimental results (Kimzey, 1977).

Figure 2.15: Illustration of the circumferential crossflow model with the orifice flow analogy (Shahrokhi, 1995).

Figure 2.16: Compressor performance prediction for one/revolution circumferential distortion screen at $85 \%$ corrected rotor speed (Shahrokhi, 1995).

Figure 2.17: Compressor performance prediction for tip-radial distortion screen at $85 \%$ corrected rotor speed (Shahrokhi, 1995).

Figure 2.18: First of a sequence of velocity vectors on top of static pressure contours. Simulated fan upstream of splitter. Core exit boundary ramped from Mach $=0.3362$ to $\mathrm{Mach}=020.2$ (Lindau, 1995)

Figure 2.19: Inner region of a multiblock grid for the Energy Efficient Engine simulation. For clarity, every other point is plotted. The entire computational grid contains 90,128 cells in 19 blocks (Stewart, 1995).....131

Figure 2.20: Energy Efficient Engine absolute frame Mach contours (in tenths) at the cruise design point, free-stream Mach number $=0.8,10.7 \mathrm{~km}$ (Stewart, 1995).

Figure 2.21: Modeling of the blade rows in the physical plane (Billet, 1988). 132 
Figure 2.22: Inlet total pressure distortion (Billet, 1988)............................................132

Figure 2.23: Azimuthal flow angle distribution. (a) In front of the rotor.

(b) Downstream of the rotor (Billet, 1988).

Figure 2.24: The Integrated Flowfield Computational Model (IFCM) for a general compression system model (Tan, 1985)

Figure 2.25: Axial velocity at IGV inlet: flow coefficient $=0.5$ (Tan, to be published).

Figure 2.26: Static pressure at IGV inlet: flow coefficient $=0.5$ (Tan, to be published).

Figure 3.1: Overall TEACC methodology

Figure 3.2: Original SLCC with overall geometry and far-field boundary conditions..135

Figure 3.3: Modifications to SLCC for calculating turbomachinery source terms........135

Figure 3.4: Methodology for calculating turbomachinery source terms. 136

Figure 3.5: Cylindrical grid used by TEACC for turbomachinery with axial grid packed through the blades and equally spaced circumferential segments.

Figure 3.6: Inlet boundary condition. 138

Figure 3.7: Exit boundary condition 138

Figure 3.8: Wall boundary condition 138

Figure 3.9: Rotationally periodic (wrap-around) boundary condition 139

Figure 3.10: Rotationally cyclic boundary condition 139

Figure 3.11: TEACC solution procedure flow chart 140 
Figure 4.1: Radial distribution of turbomachinery source terms defined at the centers of the SLCC control volumes are interpolated to the centers of TEACC's fixed-grid control volumes

Figure 4.2: Circumferential distribution of turbomachinery source terms based upon applying the SLCC separately for each circumferential segment

Figure 4.3: Axial distribution of turbomachinery source terms.

Figure 5.1: A schematic of the Rotor 1B test rig showing the reference plane locations where experimental data were taken

Figure 5.2: 90-degree, one-per-revolution distortion screen

Figure 6.1: Axial-radial view of the three-dimensional base grid modeling Rotor 1B and the test facility, with grid nodes $(69 \times 13 \times 26)$.

Figure 6.2: Radial-circumferential view of the three-dimensional base grid modeling Rotor 1B and the test facility, with grid nodes (69 $\times 13 \times 26)$ 146

Figure 6.3: Clean inlet, overall verification of TEACC with Rotor 1B 147

Figure 6.4: Clean inlet, overall total pressure ratio compared with experimental data.148

Figure 6.5: Clean inlet, overall efficiency compared with experimental data. 149

Figure 6.6: Clean inlet, exit total pressure on the $100 \%$ corrected speed line near the design throttle line.

Figure 6.7: Clean inlet, total pressure ratio on the $100 \%$ corrected speed line near the design throttle line.

Figure 6.8: Clean inlet, exit Mach number on the $100 \%$ corrected speed line near the design throttle line.

Figure 6.9: Clean inlet, exit static pressure on the $100 \%$ corrected speed line near the design throttle line.

Figure 6.10: Clean inlet, exit total temperature on the $100 \%$ corrected speed line near the design throttle line. 
Figure 6.11: Clean inlet, total temperature ratio on the $100 \%$ corrected speed line near the design throttle line

Figure 6.12: Clean inlet, exit static temperature on the $100 \%$ corrected speed line near the design throttle line.

Figure 6.13: Clean inlet, exit static density on the $100 \%$ corrected speed line near the design throttle line.

Figure 6.14: Clean inlet, exit absolute velocity on the $100 \%$ corrected speed line near the design throttle line.

Figure 6.15: Clean inlet, exit circumferential velocity on the $100 \%$ corrected speed line near the design throttle line...

Figure 7.1: Distortion screen modeling necessary to simulate the loss in total pressure across the screen; Pinker (1967)

Figure 7.2: Inlet total pressure distortion produced by the screen simulation at $100 \%$ corrected speed.

Figure 7.3: Distorted inlet, overall performance map with total pressure ratio.

Figure 7.4: Distorted inlet, overall performance map with efficiency. .158

Figure 7.5: Distorted inlet, exit total pressure on $100 \%$ corrected speed. .159

Figure 7.6: Distorted inlet, exit total temperature on $100 \%$ corrected speed. .160

Figure 8.1: Clean inlet, axial force per volume on the $100 \%$ corrected speed near the design throttle line.

Figure 8.2: Clean inlet, circumferential force per volume on the $100 \%$ corrected speed near the design throttle line.

Figure 8.3: Clean inlet, radial force per volume on the $100 \%$ corrected speed line near the design throttle line.

Figure 8.4: Clean inlet, rate of shaft work per volume on the $100 \%$ corrected speed line near the design throttle line. 
Figure 8.5: Clean inlet, radial distribution of axial force on a 15-degree circumferential segment on the $100 \%$ corrected-speed line.

Figure 8.6: Clean inlet, radial distribution of circumferential force on a 15-degree circumferential segment on the $100 \%$ corrected speed line.

Figure 8.7: Clean inlet, radial distribution of radial force on a 15-degree circumferential segment on the $100 \%$ corrected speed line.

Figure 8.8: Clean inlet, rate of shaft work on a 15-degree circumferential segment on the $100 \%$ corrected speed line.

Figure 8.9: Clean inlet, inlet and exit axial velocity on the $100 \%$ corrected speed line near the throttle line.

Figure 8.10: Clean inlet, inlet and exit radial velocity on the $100 \%$ corrected speed line near the throttle line.

Figure 8.11: Distorted inlet, axial force per volume on the $100 \%$ corrected speed line

Figure 8.12: Distorted inlet, radial force per volume on the $100 \%$ corrected speed line.

Figure 8.13: Distorted inlet, circumferential force per volume on the $100 \%$ corrected speed line.

Figure 8.14: Distorted inlet, rate of shaft work per volume on the $100 \%$ corrected speed line

Figure 8.15: Distorted inlet, screen exit total pressure on the $100 \%$ corrected speed line

Figure 8.16: Distorted inlet, screen inlet static pressure on the $100 \%$ corrected speed line

Figure 8.17: Distorted inlet, screen inlet absolute velocity on the $100 \%$ corrected speed line 
Figure 8.18: Distorted inlet, screen exit static pressure on the $100 \%$ corrected speed line.

Figure 8.19: Distorted inlet, screen inlet Mach number on the $100 \%$ corrected speed line.

Figure 8.20: Distorted inlet, screen exit Mach number on the $100 \%$ corrected speed line.

Figure 8.21: Distorted inlet, screen exit absolute circumferential velocity on the $100 \%$ corrected speed line.

Figure 8.22: Schematic of circumferential flow about the plane of symmetry bisecting the inlet distortion.

Figure 8.23: Loss in total pressure produced by the 90-degree, one-per-revolution screen on the $100 \%$ corrected speed line.

Figure 8.24: Exit total pressure distribution produced by 90 -degree, one-per-revolution screen on the $100 \%$ corrected speed line.

Figure 8.25: Exit total temperature distribution produced by 90 -degree, one-perrevolution screen on the $100 \%$ corrected speed line.

Figure 8.26: Exit static pressure distribution produced by 90 -degree, one-per-revolution screen on the $100 \%$ corrected speed line.

Figure 8.27: Exit absolute circumferential velocity distribution produced by 90 -degree, one-per-revolution screen on the $100 \%$ corrected speed line.

Figure 8.28: Streamline showing total pressure convected with the flow on the $100 \%$ corrected speed line with inlet distortion.

Figure 8.29: Circumferential transport of total temperature and total pressure through the compressor on the $100 \%$ corrected speed line with inlet distortion .175

Figure 8.30: Distorted inlet, exit total temperature on the $100 \%$ corrected speed line. 
Figure 8.31: Distorted inlet, total temperature ratio on the $100 \%$ corrected speed line.

Figure 8.32: Distorted inlet, exit total pressure on the $100 \%$ corrected speed line 177

Figure 8.33: Distorted inlet, total pressure ratio on the $100 \%$ corrected speed line .177

Figure 8.34: Distorted inlet, inlet circumferential velocity on the $100 \%$ corrected speed line

Figure 8.35: Distorted inlet, exit circumferential velocity on the $100 \%$ corrected speed line

Figure 8.36: Distorted inlet, inlet velocity on the $100 \%$ corrected speed line 179

Figure 8.37: Distorted inlet, exit velocity on the $100 \%$ corrected speed line

Figure 8.38: Distorted inlet, exit Mach number on the 100\% corrected speed line 180

Figure 8.39: Distorted inlet, inlet static pressure on the $100 \%$ corrected speed line......180

Figure 8.40: Distorted inlet, exit static pressure on the $100 \%$ corrected speed line. .181

Figure 8.41: Distorted inlet, exit static temperature on the $100 \%$ corrected speed line.

Figure D.1: Cartesian control volumes defined by Rotor 1B's geometry (circumferential segment, blade, and casing) and solution (streamlines).

Figure D.2: Three planar views of the overall Cartesian control volume. 194

Figure D.3: Technique to calculate area and volume.

Figure E.1: Overall grid with 21 axial stations for calibrating Rotor 1B with the SLCC

Figure E.2: Loss and exit relative blade angle at 10-percent radial immersion from the outer casing tip. 
Figure E.3: Loss and exit relative blade angle at 30-percent radial immersion from the outer casing tip.

Figure E.4: Loss and exit relative blade angle at 50-percent radial immersion from the outer casing tip.

Figure E.5: Loss and exit relative blade angle at 70-percent radial immersion from the outer casing tip.

Figure E.6: Loss and exit relative blade angle at 90-percent radial immersion from the outer casing tip

Figure E.7: Blockage at 10-percent radial immersion from the outer casing tip.......213

Figure E.8: Blockage at 30-percent radial immersion from the outer casing tip.......213

Figure E.9: Blockage at 50-percent radial immersion from the outer casing tip.......214

Figure E.10: Blockage at 70-percent radial immersion from the outer casing tip.......214

Figure E.11: Blockage at 90-percent radial immersion from the outer casing tip.......215

Figure E.12: Overall comparison of the SLCC calibration with experimental data in total pressure ratio and efficiency for $50,70,90$, and $100 \%$ speed.

Figure E.13: Calibration, total pressure ratio on the $100 \%$ corrected speed line near the design throttle line

Figure E.14: Calibration, total temperature ratio on the $100 \%$ corrected speed line near the design throttle line

Figure E.15: Calibration, exit total pressure on the $100 \%$ corrected speed line near the design throttle line.

Figure E.16: Calibration, exit total temperature on the $100 \%$ corrected speed line near the design throttle line.

Figure E.17: Calibration, exit static pressure ratio on the $100 \%$ corrected speed line near the design throttle line 
Figure E.18: Calibration, exit static temperature ratio on the $100 \%$ corrected speed line near the design throttle line

Figure E.19: Calibration, exit velocity on the $100 \%$ corrected speed line near the design throttle line.

Figure E.20: Calibration, exit Mach number on the $100 \%$ corrected speed line near the design throttle line

Figure E.21: Calibration, exit circumferential velocity on the $100 \%$ corrected speed line near the design throttle line.

Figure E.22: Calibration, exit static density on the $100 \%$ corrected speed line near the design throttle line.

Figure E.23: Calibration, inlet relative velocity on the $100 \%$ corrected speed line near the design throttle line.

Figure E.24: Calibration, exit relative velocity on the $100 \%$ corrected speed line near the design throttle line

Figure E.25: Calibration, inlet relative Mach number on the $100 \%$ corrected speed line near the design throttle line.

Figure E.26: Calibration, exit relative Mach number on the $100 \%$ corrected speed line near the design throttle line.

Figure E.27: Calibration, inlet relative circumferential velocity on the $100 \%$ corrected speed line near the design throttle line.

Figure E.28: Calibration, exit relative circumferential velocity on the $100 \%$ corrected speed line near the design throttle line.

Figure F.1: Axial-radial view of grid doubled in the axial direction from the base grid with grid nodes $(131 \times 13 \times 26)$.

Figure F.2: Axial-radial view of the grid doubled in the radial direction from the base grid with nodes $(69 \times 24 \times 26)$ 
Figure F.3: Radial-circumferential view of the three-dimensional base grid $(69 \times 13 \times 26)$

Figure F.4: Radial-circumferential view of the grid doubled in the radial direction from the base grid with nodes $(69 \times 24 \times 26)$.

Figure F.5: Radial-circumferential view of the grid doubled in the circumferential direction from the base grid with nodes $(69 \times 13 \times 50)$.

Figure F.6: Area correction for converting to Cartesian control volumes.

Figure F.7: Percent difference of total temperature ratio with experimental data for the base grid and the grid doubled in the axial, radial, and circumferential direction

Figure F.8: Percent difference of total pressure ratio with experimental data for the base grid and the grid doubled in the axial, radial, and circumferential direction

Figure F.9: Percent difference of exit absolute velocity with experimental data for the base grid and the grid doubled in the axial, radial, and circumferential direction.

Figure F.10: Percent difference of exit circumferential velocity with experimental data for the base grid and the grid doubled in the axial, radial, and circumferential direction

Figure F.11: Percent difference of exit static pressure with experimental data for the base grid and the grid doubled in the axial, radial, and circumferential direction. .228

Figure F.12: Percent difference of exit static temperature with experimental data for the base grid and the grid doubled in the axial, radial, and circumferential direction. .228 


\section{Nomenclature}

\begin{tabular}{ll} 
Symbol & Definition \\
a & Local speed of sound \\
A & Area \\
A & Component of area \\
c & Constant pressure specific heat \\
d & Distribution function \\
e & Internal energy and kinetic energy per mass \\
E, F, G & Flux Vectors \\
F & Force \\
F & Component of force \\
J & Jacobian \\
k & Curvature \\
m & Rate of mass flow \\
n & Number of nodes \\
N & Rotor speed \\
P & Pressure \\
Q & Conservation Variables \\
r & Radial direction \\
\hline
\end{tabular}


$\ddot{\mathrm{r}}$

$\mathrm{R}$

Residual Explicit part (RHS) of the discretized conservation equations

S

S

Sì

$\mathrm{t}$

$\mathrm{T}$

$\mathrm{u}$

$\mathrm{v}$

V

V

$\forall$

w

$x, y, z$

$\alpha$

$\gamma$

$\delta$

$\omega$

Translational acceleration

Ideal gas constant

Source vector

Component of the source vector

Rate of shaft work

Time

Temperature

Velocity in the $\mathrm{x}$-direction

Velocity in the $y$-direction

Velocity

Component of velocity

Volume

Velocity in the z-direction

Cartesian right-handed coordinate directions

Absolute swirl angle

Ratio of specific heats

Ratio of local volume to cumulative volume through the blade row

Weighting function 

$\underline{\text { Symbol }}$
$\underline{\text { Definition }}$
$\rho$
Density
$\tau$
Nondimensional time
$\theta \quad$ Circumferential direction
$\xi, \eta, \zeta \quad$ Directions in generalized curvilinear coordinates

\section{Subscripts}

b

B Bottom face of control volume

c

cir

E

g

$\mathrm{h}$

i

I

$\mathrm{J}, \mathrm{K}, \mathrm{L} \quad$ Integer coordinates of physical grid nodes

q

ref

$\mathrm{R}$

segment

$\mathrm{t}$

Bleed

Corrected to standard day conditions

Cylindrical cross section

Exit face of control volume

Counting index

Inlet face to control volume

Standard day reference condition

Right face of control volume

Circumferential segment

Total
Index identifying each radial control volume in the bladed region

Index identifying mass bleeds, forces or rate of shaft work

Index identifying the residual vector at each node in the flow field 


$\begin{array}{ll}\text { Symbol } & \text { Definition } \\ \text { tri } & \text { Triangular cross section } \\ \mathrm{T} & \text { Top face of control volume } \\ \mathrm{w} & \text { Circumferential direction } \\ \mathrm{x}, \mathrm{y}, \mathrm{z} & \text { Directions in Cartesian coordinates }\end{array}$

\section{$\underline{\text { Superscripts }}$}

$\begin{array}{ll}\text { P } & \text { Previous value in time } \\ \text { Relax } & \text { Nontransformed conservation variables } \\ \text { SLCC } & \text { Base variable adjusted by relaxation factor } \\ \end{array}$

Nomenclature for SLCC in Appendix C

Nomenclature for control volume geometry in Appendix D 


\subsection{Introduction}

Modern high-performance military aircraft are frequently subjected to rapid flight maneuvers which place great operational demands on their components. One component that is particularly sensitive to the fluid dynamic transients produced by these demands is the air-breathing gas turbine engine. Although the engine is typically designed to operate in a stable manner during all aspects of flight and produce adequate thrust with low vibration and minimum fuel consumption, high angles of attack can result in highly distorted flow fields entering the engine that generally reduce the engine compressor stability margin.

Inlet distortion is usually quantified by measuring profiles of total pressure and total temperature at the aerodynamic interface plane (AIP) in front of the compressor, as shown in Figure 1.1. To emphasize how inlet distortion varies with flight, a series of pressure distortion patterns obtained in a McDonnell-Dougless F-15 inlet tested at Arnold Engineering Development Center (AEDC) and operated at transonic conditions are presented in Figure 1.2 (Beale,1993). For a straight and level flight condition (Figure 1.2a) the inlet produces a radial inlet distribution pattern with a maximum 10-percent loss in total pressure. With an angle of attack (ALPHA) of 30-degrees (Figure 1.2b), the distortion pattern at the AIP becomes complex with a 10-percent maximum loss in total pressure. Yet another complex distortion pattern is generated by altering the flight conditions with an angle of sideslip (BETA) of 10-degrees (Figure 1.2c). These 
changing, complex inlet distortion patterns suggest the need to understand their effects on compressor performance to avoid operability problems.

Since total pressure distortion is the primary driver of the engine to its stability limit, independent of other causes, we should develop further understanding of its origin. A fixed-geometry inlet is designed with trade-offs; consequently, some engine/inlet compatibility problems are to be expected at off-design conditions. However, these trade-offs generate inlet-distorted flow which is intensified by rapid aircraft maneuvers. Although variable inlet geometry can improve engine/inlet compatibility at off-design conditions, thereby reducing the inlet distortion, it carries a severe weight penalty with it. Many current military aircraft are designed with sharp inlet edges to minimize shock losses under supersonic flow, but at low speeds the flow tends to separate at the lip especially during violent flight maneuvers. Separated inlet flow can be unsteady and produce highly distorted flow at the compressor face. In addition, supersonic inlets introduce a variety of oblique shock waves designed to efficiently reduce the Mach number in the diffuser. These shock waves interact with the unsteady boundary layer and the unsteady ambient atmosphere to form unsteady shocks which deliver inlet total pressure distortion to the engine face. Even when unsteady effects are mild, off-design flight can cause lags in the control system. Therefore, engine/inlet incompatibility arises as a result of some variables being off-schedule during the transient, leading to stall margin reduction (Bouillet, 1974). 
Total pressure inlet distortion lowers compressor stall margin because it increases the angle-of-attack on the compressor blades. This flow phenomenon is illustrated in Figure 1.3 (Davis, 1991). The clean inlet stall line is presented for reference and a new stability limit stall line is produced by inlet distortion. Only a 2-percent loss in stall margin is observed at the low corrected speed, but the high corrected speed produces a stall margin loss of 10-percent. High distortion levels may cause the engine to surge (Figure 1.4a) at high rotational speeds or slip into rotating stall (Figure 1.4b) at lower rotational speeds. The effects of inlet distortion on a given compressor must be understood to ensure that adequate stability margin is maintained during all aspects of an aircraft's flight.

Greitzer and Strand (1978) demonstrated that distortion imposed on a circumferentially swirling flow has a three-dimensional (3D) nature. This was demonstrated experimentally by Huard (1978), who placed a classical variable-porosity distortion screen in front of an inlet guide vane (IGV). The IGV was located inside a constant radius, cylindrical annulus channel. Without inlet distortion imposed on the flow, the inlet guide vanes produced uniform wrapping streamlines with no noticeable 3D effects. With the inlet distortion imposed upstream of the inlet guide vane, however, strong 3D effects were observed. In the high-pressure region of the distorted flow, the streamlines were deflected radially to the outer casing. In the low-pressure region of the distorted flow, the streamlines were displaced radially toward the inner casing. 
Because three-dimensional effects are fundamental to the development of both inlets and compressors, design or analysis engineers must understand the details of the flow field to determine the effects of inlet total pressure distortion on the compressor. Although distortion effects can be quantified in a ground test facility, the inlet and engine are currently tested separately because the aircraft fuselage, inlet, and engine are typically too large to fit in a wind tunnel. Thus, a forebody simulator is used in conjunction with the inlet to characterize its flow field. The forebody simulator produces a flow field at the inlet reference plane (IRP) similar to the flow field produced by the aircraft (Figure 1.5). The flow is usually measured at the AIP by a 40 -probe pressure rake that not only captures the spatial distortion but its unsteady nature as well. Based upon the SAE (AIR1419,1983 ) which states that an unsteady distortion pattern can be characterized by snapshots of the pattern at a discrete time, time-variant distortion is reduced to a set of steady-state patterns. Screens are constructed to capture the most severe dynamic patterns produced by the inlet and then placed in front of the engine, to measure the loss of stall margin produced by the steady-state inlet distortion (AIR-1419, 1983). However, instrumenting a compressor and performing the necessary number of tests to adequately understand the flow field produced and the resulting operational characteristics of the compressor is extremely expensive. A computational fluid dynamics (CFD) simulation would be very useful in the design and analysis phase, since it gives additional information with inexpensive modifications. A validated CFD simulation would also be useful for tailoring distortion testing to fewer and more precise tests. Such a simulation 
would allow the extension of hardware boundaries to perform "what if" studies, or it could be combined with structural simulations to evaluate the effect of inlet distortion on the structural integrity of the engine or adjacent structures. 


\subsection{Previous Work}

Researchers have been developing computer simulations to understand the complicated flow environment of the compressor for many years. Many of these simulations were developed for analyzing specific characteristics of turbomachinery, such as reduction of the stability margin due to inlet distortion, complicated three-dimensional secondary flows due to rotor end wall effects, and unsteady wakes produced behind a rotating blade row. This review focuses on inlet distortion simulations and some axialradial simulations that could be extended in the circumferential direction to investigate inlet distortion.

The research community is also investigating three-dimensional blade passage simulations. The mixing plane and the average-passage model development are oriented toward steady-state results (Adamczyk, 1984), while the time-resolved unsteady simulations are being driven toward a completely unsteady result (Denton, 1992; Dawes, 1985, 1992; Ni, 1989; Giles, 1988; Rao, 1990; Rai, 1987; Jorgenson, 1989). Although the modeling techniques developed in these simulations are very. useful, they typically require too much computational time and computer resources for a direct application to inlet distortion.

The review of previous work is separated into the following two categories of research simulations: (1) parallel compressor and (2) semi-actuator disc. The simulations presented in each category have been restricted to $2 \mathrm{D}$ simulations which significantly contributed to blade modeling techniques or to $3 \mathrm{D}$ simulations which are 
actually used to analyze the effect of inlet distortion. Parallel compressor models (category 1) are limited by their inability to conservatively pass information between adjacent segments. Semi-actuator disc models (category 2) represent the most significant contributions to the foundation of this dissertation, with $2 \mathrm{D}$ blade modeling techniques and 3D inlet distortion simulations.

\subsection{Parallel Compressor Simulation}

In classical parallel compressor theory (Pearson, 1963), the compressor is separated into circumferential segments extending axially through the compressor (Figure 2.1a). Each segment acts in parallel with all other segments, exiting to a common boundary condition. The compressor is modeled separately in each segment with the same one-dimensional (1D), steady-state characteristics. Since a steady-state or transient inlet distortion can be established at the inlet, each circumferential segment will operate independently at different locations on the same characteristic. For example, a 180degree distortion is imposed at the inlet of a parallel compressor simulation in Figure 2.1b. The segment with the clean inlet (higher inlet total pressure) behaves as though the entire compressor operated on the compressor map free from inlet distortion. The low inlet total pressure segment behaves as though the entire compressor operates on the same characteristic at a reduced corrected mass flow for the larger total pressure ratio. A mean operating point is defined as the mass-weighted average of the total pressure ratio associated with each segment. System instability is assumed to occur when either of the segments becomes unstable, and instability is defined when the low-velocity segment 
reaches the uniform flow (undistorted) stall point. A significant weakness of this simulation is the absence of mass and momentum transfer between circumferential segments. It is generally known that the classical parallel compressor simulation is too conservative at predicting the stability limit (Korn, 1974; Steenken, 1983; Mazzawy, 1977; Kimzey, 1977; Shahrokhi, 1995).

\subsubsection{Modifications to Parallel Compressor Simulations}

Many modifications have been incorporated to improve the accuracy of the classical parallel compressor theory (PCT). These compressor simulation modifications were usually represented by some form of inherently one-dimensional steady-state characteristic, and even though mass transfer in some cases was accounted for, momentum transfer in the circumferential and radial direction was ignored. These models, however, have provided good understanding of distortion and the prediction of stability loss margin. Seven such efforts are described herein.

(1) Korn (1974) modified the parallel compressor simulation by incorporating a dynamic transition time for a segment to change from unstalled to stalled when the engine neared the stability limit, arguing that the change to a dynamic event is not instantaneous. His analysis was based upon a single rotor with a 180-degree total pressure inlet distortion imposed between the 90- to 270-degree circumferential extent. Ideally, the blades passing through the sudden discontinuity in total pressure would produce the sudden discontinuous increase in incidence as shown in Figure 2.2a. However, the incidence in the distorted region was observed experimentally to be greater 
than the steady-state stalling incidence (SS Stall) without causing the compressor to stall. The rotor incidence was found to vary in the vicinity of stall with a frequency related to the distortion extent and rotational speed. Korn determined that because of the lift response delay, the rotor operated stably with an incidence greater than the stalling incidence provided the change in incidence did not persist for long. The parallel compressor simulation was modified by extending the dynamic characteristics into the stalled region, as shown in Figure 2.2b. The parallel compressor theory (PCT) produced a very conservative stall margin, but Korn's modified parallel compressor theory (MPCT) accurately estimated the stability limit for a single rotor. This same degree of success was not realized in multi-blade row machines.

(2) Steenken (1983) modified the parallel compressor theory by developing models for the transfer of mass, momentum, and energy before and after the compressor. He observed classical parallel compressor theory (CPCT) predictions for the loss of surge pressure ratio to be most conservative at estimating the stability limit for the low corrected speed characteristics which are much flatter than the higher corrected speed characteristics. Steenken argued that the loss in stall margin could not be attributed to the redistribution of flow within modern axial-flow compressors because the gap between rotors and stators was too small. Rather, the underprediction of stall margin must be associated with the redistribution of the flow field upstream and downstream of the compressor. Therefore, he developed a 2D axial-circumferential duct flow simulation 
and employed it to model the blade-free volumes on both sides of the compressor with the compressor modeled by classical parallel compressor theory.

Steenken applied this model using the circumferential-redistribution parallelcompressor on a complete fan component test rig (Figure 2.3). The annulus was divided into six equal sectors, with three sectors located within the 180-degree low-total-pressure square-wave distortion, and the remaining three sectors located in the high-total-pressure region. The performance of the compressor for estimating the stability limit is presented in Figure 2.4 with an "open triangle." An improvement over classical parallel compressor theory was obtained for two reasons: (1) a more realistic inlet flow field was obtained, particularly in terms of compressor entrance incidence, and (2) the parallel compressor exited to a nonuniform back boundary condition which is more realistic.

(3) Mazzawy $(1976,1977)$ extended the classical parallel compressor theory by including multiple circumferential segments. He modeled the performance of each undistorted blade row separately with steady-state characteristics, as in classical parallel compressor theory. However, for the distorted region he developed additional characteristics to account for unsteady effects and distortion-induced inlet flow redistribution. He observed that the uniform exit static pressure boundary condition produced poor answers when it exited to a diffuser or another compressor in a multispool configuration. This led him to develop an iterative procedure to calculate a circumferential distribution of exit static pressure which satisfied the specification of total mass flow. The nonuniform distribution of exit static pressure required the 
development of a procedure for tracking the circumferential displacement of each segment of fluid through the compressor, as shown in Figure 2.5. The approach was based upon the argument that the flow through the compressor is seldom axial. Mazzawy modeled the flow by circumferentially shifting the segments to account for the flow angularity and the additional displacement associated with the rotor rotation (circumferential particle swirl). Mazzawy further improved the prediction of the classical parallel compressor stability limit by including dynamic effects. He observed from experimental data that the steady-state stability limit could be exceeded until the static pressure ratio-flow characteristic peaked and became positively sloped. The new stability limit was easily incorporated into the modified parallel compressor simulation since each circumferential segment already had a potentially different static pressure flow characteristic.

Mazzawy also recognized the difficulty of obtaining individual blade row characteristics and simplified his simulation by using overall compressor performance characteristics. Approximations were incorporated using the overall characteristics to retain the effects of unsteady flow, circumferential particle swirl, and upstream flow redistribution. These modifications were applied to a high-speed (tip speed $=1,600$ $\mathrm{ft} / \mathrm{sec}$ ) single-stage fan, which was subjected to a 120-degree inlet total pressure distortion. The amplitude of the distortion for the test case was greater than 20-percent of the mean inlet total pressure. The results of the model with individual blade row characteristics and the overall compressor performance characteristics are presented with 
experimental data in Figure 2.6. A comparison of results reveals an improvement in the blade row performance model relative to the overall compressor performance model. However, the results are restricted to the axial-circumferential averaged domain, and no information is revealed about the radial distribution of the effects of inlet distortion.

(4) Longley and Greitzer (1992) investigated the basic ideas for more appropiately modeling the nonuniform flow field in the blade-free region on either side of the compressor. They investigated the nonuniform flow field upstream of the compressor by modeling the compressor with classical parallel compressor theory and a uniform exit static pressure (equivalent to exiting to a uniform duct). A 180-degree total pressure inlet distortion was imposed far upstream (far-field) of the compressor in Figure 2.7a. They argued that total pressure is a convected quantity upstream of the compressor since viscous effects can often be ignored in light of the overall distortion velocity distribution. This means the imposed total pressure inlet distribution does not change from the farfield to the entrance of the compressor. At the far-field where the inlet distortion was imposed, the static pressure was uniform, but at the compressor inlet a nonuniform static pressure (Figure 2.7b) was produced by the compressor because it exits to a uniform static pressure. Therefore, the upstream static pressure distribution produced by the compressor operating with inlet distortion will produce a flow redistribution in front of the compressor. The flow redistribution is shown in Figure $2.7 \mathrm{c}$ where the axial velocity has attenuated from the far-field to the inlet of the compressor face due to the change in uniform static pressure from the far-field to the inlet of the compressor. 
(5) Longley and Greitzer (1992) also explored the effect of adding the passive components, diffuser and nozzle, downstream of the compressor to emphasize the potential effect of downstream components. The investigation was conducted at low speed using a three-stage compressor with a 180-degree inlet total pressure distortion. First connected a diffuser (short enough to be modeled as a parallel diffuser) downstream of the compressor which was modeled by parallel compressor theory as shown in Figure 2.8. Total pressure inlet distortion was imposed upstream of the compressor, establishing a high and low total pressure stream leaving the compressor and entering the diffuser. Since both streams exit the diffuser into uniform static pressure, the stream with the low total pressure (low velocity) entering the diffuser will have the highest static pressure. The converse was true when the diffuser was replaced with a nozzle at the exit of the compressor where the low total pressure stream had the lower static pressure at the compressor exit. A comparison was made with compressor performance for the inclusion of a diffuser, constant area duct, and nozzle in Figures 2.9 and 2.10 when each was subject to the same inlet total pressure distortion. The researchers found that a compressor with a diffuser attached was most likely to have stability problems, but a compressor with a nozzle attached was the more stable. Their results revealed a nonuniform exit static pressure to be in phase with the total pressure inlet distortion at the nozzle, out of phase for the diffuser, and nearly zero for the constant area duct. This work stressed the importance of proper modeling of the downstream component of the compressor. 
(6) Kimzey (1977) sought to improve the classical parallel compressor model by deriving from first principles a general set of time-dependent, three-dimensional relationships to model flow in the compressor. These relationships were applied to a three-dimensional grid structure tailored to model turbomachinery with the compressor broken up axially, radially, and circumferentially. However, due to the lack of radial and circumferential characteristics to model turbomachinery the radial and circumferential momentum equations were eliminated and replaced with empirical relationships. The axial momentum equation was retained with stage-by-stage compressor characteristics used to model turbomachinery. Classical parallel compressor theory was further refined by the inclusion of radial and circumferential mass redistribution and a radial work redistribution from empirical relations. In addition, a first-order lagging function was incorporated to improve the model's ability to predict stall margin.

Kimzey applied the simulation to the multiple blade row compressor, XC-1. Pure circumferential and pure radial total pressure inlet distortion comparisons each were made with experimental data. Since specific experimental data were not available from the $\mathrm{XC}-1$ to make these comparisons, other compressor test data were used to check the model results for proper trend and order of magnitude. Figure 2.11 shows the effect of increasing the severity of a 60-degree arc of circumferential total pressure distortion. Distortion pattern magnitudes of $5,10,15$, and $20 \%$ were computed. Increasing loss of stability limit pressure ratio and airflow was observed. As shown in Figure 2.12, the model results were compared to a range of experimental data from other compressors 
giving reasonable results. The effects of tip radial distortion (low pressure in the tip region) are illustrated in Figure 2.13 where the $\mathrm{XC}-1$ compressor was loaded to the stability limit. Pattern severities of 5, 10, and $20 \%$ were considered. Figure 2.14 shows comparisons of the XC-1 model results with experimental data from other tests.

(7) Shahrokhi (1995) duplicated Kimzey's modeling approach into a current-day compressor model, DYNTECC (Hale, 1992), and compared his results with a different compressor. Shahrokhi observed that circumferential inlet total pressure distortion increased mass flow in the direction of rotor rotation. Static pressure differences were generated between circumferential segments which could drive the flow between the rotor and stator. This circumferential mass flow was modeled by algebraic expressions based upon classic orifice flow (Figure 2.15). Shahrokhi modeled the mass flow in the radial, or crossflow, direction based upon the principle of a variation in static pressure driving flow in the radial direction. This led to an algebraic relationship based upon the steadystate incompressible radial momentum equation. He further included an algebraic relation based upon scale factors to account for the nonuniform radial distribution of blade loading. An improvement over classical parallel compressor theory was obtained with circumferential inlet distortion; however, radial improvement was difficult to quantify since multiple empirical scale factors were arbitrarily selected.

Shahrokhi compared his simulation to data from a two-stage, low-aspect-ratio fan for both pure circumferential and pure radial total pressure inlet distortion. To model the one-per-revolution inlet distortion, the parallel compressor simulation was divided into 
eight circumferential segments. His results for pure circumferential inlet distortion are compared with experimental data and classical parallel compressor theory in Figure 2.16 at 85-percent corrected speed. A significant improvement was achieved over classical parallel compressor theory. The stability limit was predicted within $2.4 \%$ in compressor airflow and $1.5 \%$ in overall compressor pressure ratio for the 85 -percent full speedline. The second stage was determined to be the critical stalling stage, since it was the first stage to reach its stability limit. Shahrokhi also made a comparison with experimental data for a pure radial pattern on the 85-percent corrected speedline. The tip-radial distortion produced a low-pressure region at the blade tip region of the compressor inlet, and a relatively clean inlet flow condition was maintained at the hub region of the compressor inlet. The results of his investigation are presented in Figure 2.17, where the predicted compressor performance was within 1.42-percent for corrected airflow and 0.61-percent for overall compressor pressure ratio, as compared to experimental data. This simulation required radial crossflow coefficients to be wildly adjusted to calibrate the model to experimental data.

Parallel compressor theory has been valuable for investigating overall fundamental compressor operability and performance issues. The effects of complicated physical three-dimensional phenomena were innovatively modeled by the above investigators in order to improve this theory. Nowhere was this better seen than those efforts which improved the compressor model by allowing crossflow between segments in order to estimate the flow field on either side of the compressor more accurately. The 
next category of simulations removes some of the modeling restrictions incorporated in parallel compressor theory by using first principles to pass information multidimensionally in the radial direction.

\subsection{Semi-Actuator Disc Theory}

Semi-actuator disc theory provides greater accuracy in compressor performance and stability loss margin, compared to parallel compressor theory, since it incorporates coupled mass and momentum transfer between segments. A compressor can be simulated with semi-actuator disc theory by replacing the blade geometry with an externally imposed discontinuity in flow properties over the axial spacing of the bladed region. These discontinuities also bring about a discontinuity in one or more of the conservative properties within the flow field. The advantage of semi-actuator disc theory is that the flow-field geometry often can be greatly simplified, thus minimizing expensive computational resources.

\subsubsection{Two-Dimensional Compressor Simulations}

Lindau (1995) developed an axial-radial 2D dynamic compressor simulation. The primary focus of his research was to model the splitter plane of a turbofan engine, splitting the fan flow to the high-pressure compressor and the bypass duct and examining the flow in this area during compressor surge (Figure 2.18). Lindau used a flux difference splitting algorithm to solve the discretized Euler equations which were modified to include turbomachinery source terms within the blade rows. The source terms were calculated from empirical information available from 1D compressor maps 
(Davis, 1986). A radial distribution of 1D maps can be supplied by the user for each of the radial control volumes within the bladed region to model secondary flows; however, these radial distributions are usually not available. Lindau developed an algebraic radial distribution of source terms based upon blade passage flows found in the open literature. The forces and shaft work done on the fluid by the compressor would be unchanged for a slug of fluid, but the sources were assumed to be greater at the middle of the blade passage than at the hub or tip. Lindau made no comparisons with experimental data to validate this model.

Stewart (1995) developed a two-dimensional simulation including the internal and external flow path to simulate engine performance. He developed an axisymmetric engine model capable of modeling compressible rotational flow; this allows entropy and vorticity production using the Euler equations with turbomachinery source terms. A torque equation was included for each shaft to maintain proper rotor dynamics during transient simulations. A streamline curvature procedure was used to calculate the blade source terms, and combustors, mixers, and bleeds were simulated based on available data. The axisymmetric equations were discretized along with the turbomachinery source terms and advanced to steady state using a four-stage Runge-Kutta algorithm with artificial first- or third-order dissipation applied for stability.

Stewart evaluated his results on the Energy Efficient Engine (EEE) designed and tested by General Electric in the early 1980's. The grid, with 90,128 grid cells describing the inner region is presented in Figure 2.19. The flight condition was the engine's cruise 
design point, an inlet Mach number of 0.8. Mach number contours from Stewart's results are presented in Figure 2.20 for EEE's cruise design point. A cycle deck tailored to model EEE was used to evaluate the simulation's performance. The comparison of Stewart's simulation with the cycle deck agreed within 2-percent on mass flow, total pressure, and total temperature. The two simulations agreed on engine net thrust to within 3-percent. The largest discrepancies occurred within the compressor and the turbine simulation, where total pressure differences were as large as 20-percent.

\subsubsection{Three-Dimensional Distortion Simulations}

Billet, et al. (1984, 1985, 1988; Bry, 1985; Joubert, 1990) developed a threedimensional compressible, time dependent, and non-uniform axial compressor simulation to investigate steady distorted flow within an annular channel (Figure 2.21). The Euler equations written in the cylindrical coordinate reference frame relative to the channel were transformed into a parallelepiped calculation domain by a linear transformation to obtain a uniform grid. All loss generation was restricted to the bladed regions. The nonbladed regions were modeled as inviscid duct control volumes. This allowed the Euler equations to be solved over the blade-free domain by a time-splitting finite-difference method while the bladed region was solved using a semi-actuator disc for both rotating and fixed blade rows.

In Billet's model, resolution of the 3D viscous flow field through the blade was too computationally expensive, so the bladed regions were modeled with a coarse axial grid (only on the blade row inlet and exit). The Navier-Stokes equations were integrated 
over a streamtube passing through a single blade passage bounded by adjacent blades in the azimuth direction, inlet and exit of the blade passage, and top and bottom by stream surfaces in the radial direction. Many flow field details were eliminated by using a coarse grid, thus only the general character of the flow field was modeled. The model required empirical information specifying total pressure-loss coefficient and the exit angle in a relative reference frame.

Billet's computed results were compared to experimental data from a test stand of a low-pressure, single-stage, axial compressor with a constant-section annular duct. The simulation's grid used a total of 78,000 grid points with 78 in the axial direction, 91 in the circumferential direction, and 11 in the radial direction. Total pressure inlet distortion was imposed one rotor diameter upstream of the compressor. The complex inlet distortion that was imposed is presented in Figure 2.22. The average inlet distortion was approximately half of the inlet dynamic pressure. One result, presented in Figure 2.23, compares the inlet and exit azimuthal flow angle plotted versus circumferential extent. The inlet azimuthal flow angle underpredicts that of the experimental data because the computational mass flow was underpredicted. There is a shift of the exit azimuthal flow angle in the circumferential direction, even though the overall absolute magnitude was captured. There were no comparisons made with a high-speed, high-pressure compression system.

Tan (1985) developed an inlet distortion simulation by modeling the flow field adjacent to and including the compressor with the time-dependent, incompressible Euler 
equations modified by the inclusion of body forces for low-speed compressors (Figure 2.24). Each blade row was modeled separately by blade forces, allowing the modeling of blade gaps if desired. The blade forces were calculated by assuming the flow field to be locally axisymmetric at each circumferential position and remaining consistent with the 3D flow field before and after the blade. These blade body forces were updated regularly so the solution of the Euler equations could maintain time accuracy. Tan argued that secondary flow, which is highly three-dimensional manifests itself in the form of twisted stream surfaces that can be accounted for by correlations. These correlations can be calculated based on classical secondary flow theory (Hawthorne, 1955; Squire, 1951; Smith, 1953) and superimposed on the calculations for the body forces.

Tan simulated the MIT three-stage compressor with inlet guide vanes (IGV) and imposed a square wave of a 20-degree total pressure inlet distortion far upstream of the compressor. Measurements were taken of the axial velocity distribution and static pressure distribution at the inlet plane of the IGV. These measurements of axial velocity and static pressure are presented in Figures 2.25 and 2.26. The overall character was captured by the simulation with a shift in the circumferential direction for axial velocity and a too severe drop in static pressure as compared to experimental data. No comparisons were made with a high-pressure compression system.

\subsection{Expected Benefit of a New. 3D Distortion Simulation}

It is clear from this brief review of previous work that the state-of-the-art turbomachinery simulation techniques developed thus far exhibit limitations that must be 
overcome in order to accurately model modern compressors, in particular to investigate the effects of inlet distortion. The parallel compressor theory simulations are unable to account for the detailed radial and circumferential passage of information which is required throughout the compressor simulation. Although two-dimensional models have been used effectively for developing and demonstrating techniques for modeling turbomachinery source terms, they are inadequate for analyzing the $3 \mathrm{D}$ nature of inlet distortion. The 3D simulations developed are restricted to low-speed machines (incompressible) or to inappropriate radial redistribution of conservation quantities.

Therefore, a CFD simulation designed to overcome these limitations has been developed and is called the Turbine Engine Analysis Compressor Code (TEACC). This code solves the compressible 3D Euler equations over a finite-volume grid domain through each blade row. The Euler equations are modified to include turbomachinery source terms which represent the effect of the blades. The turbomachinery source terms are bleed, blade forces in the three Cartesian directions, and shaft work. The source terms are calculated for each circumferential grid section of each blade row by the application of a streamline curvature code. A methodology was developed for distributing the turbomachinery source terms axially, radially, and circumferentially through the bladed region.

A brief outline of the following chapters is given to emphasize where TEACC is fully constructed and compared with experimental data for inlet distortion. Chapters 3 and 4 present the development of the overall approach for TEACC, define the 
turbomachinery source terms calculated from the SLCC, and establish the methodology for implementing the source terms into TEACC to produce a 3D turbomachinery analysis tool. The test vehicle and experimental data available for comparison with TEACC is presented in Chapter 5. Chapter 6 is a confirmation that TEACC was constructed properly by comparing clean inlet TEACC results with experimental data. The new contribution of TEACC as an inlet distortion analysis tool is demonstrated in Chapter 7, where comparisons of TEACC results are made with experimental data. The relative meaning of the source terms, the flow field around the distortion screen, and the effect of swirl on the compressor operation in the presence of total pressure inlet distortion are investigated in Chapter 8. Chapters 9 and 10 summarize the major contributions of TEACC and make recommendations to enhance TEACC's capabilities. 


\subsection{Overall Approach to TEACC}

The overall TEACC development methodology is presented graphically in Figure 3.1. A general-purpose three-dimensional flow simulation computer code (NPARC) was modified to accept turbomachinery source terms by semi-actuator disc theory. These turbomachinery source terms are calculated using a streamline curvature code (SLCC). TEACC is constructed by combining NPARC with the SLCC to produce a turbomachinery simulation with the capability of analyzing inlet distortion. The inputs to TEACC are the grid defining the flow path through the bladed region, boundary conditions, initial conditions, and turbomachinery source terms describing the effect of rotating blades. The output from TEACC is a flow field defined on each grid point which can be appropriately averaged to give radial and overall blade row-by-blade row performance of the compressor.

This chapter introduces the NPARC (Cooper, 1989) and SLCC (Hearsey, 1970) codes and shows how they have been modified and combined to produce TEACC. Redevelopment of the NPARC and SLCC algorithms are not included in this dissertation since they have been adequately developed in the references, but their basic solution procedures are described so modifications can be readily understood. TEACC uses the 3D Euler equations with turbomachinery source terms. A technique to calculate these turbomachinery source terms is presented along with the necessary modifications to the SLCC code. In addition, grid construction, boundary conditions, initial conditions, convergence criteria, and solution procedures are presented. 
TEACC was developed by modifying NPARC to include source terms. NPARC was developed, in part, at the Arnold Engineering Development Center (AEDC) as a general duct flow solver for use within the Engine Test Facility (ETF). This tool has proven capable of analyzing internal propulsion flow fields and test facilities such as those produced for the design of a thrust-reversing engine exhaust collector (Jones, 1994) and the design of a free-jet test for inlet icing studies (Bartlett, 1993). NPARC is capable of solving the full Navier-Stokes set of equations; however, the turbomachinery modeling for TEACC eliminates the viscous terms, resulting in an Euler solver.

\subsection{Governing Equations}

The governing equations for TEACC can be developed by applying the conservation of mass, momentum, and energy. Upstream and downstream of the compressor the viscous forces are negligible on the overall velocity distribution allowing these regions to be accurately modeled with the Euler equations (Longley, 1992). The viscous effects and flow turning associated with the bladed region are simulated with the Euler equation and turbomachinery source terms. The equations of fluid motion with turbomachinery source terms can be transformed from Cartesian coordinates to generalized curvilinear coordinates, non-dimensionalized, and presented in the following flux vector form:

$$
\frac{\partial \overline{\mathbf{Q}}}{\partial \tau}+\frac{\partial \overline{\boldsymbol{E}}}{\partial \xi}+\frac{\partial \overline{\boldsymbol{F}}}{\partial \eta}+\frac{\partial \overline{\mathbf{G}}}{\partial \zeta}=\overline{\mathbf{S}}
$$


where conservative variables are

$$
\overline{\mathbf{Q}}=\frac{1}{\mathrm{~J}} \mathbf{Q},
$$

the flux vectors are

$$
\begin{aligned}
& \bar{E}=\frac{1}{\mathrm{~J}}\left(\xi_{\mathrm{x}} \boldsymbol{E}+\xi_{\mathrm{y}} \boldsymbol{F}+\xi_{\mathrm{z}} \boldsymbol{G}\right), \\
& \overline{\boldsymbol{F}}=\frac{1}{\mathrm{~J}}\left(\eta_{\mathrm{x}} \boldsymbol{E}+\eta_{\mathrm{y}} \boldsymbol{F}+\eta_{\mathrm{z}} \boldsymbol{G}\right), \\
& \overline{\boldsymbol{G}}=\frac{1}{\mathrm{~J}}\left(\zeta_{\mathrm{x}} \boldsymbol{E}+\zeta_{\mathrm{y}} \boldsymbol{F}+\zeta_{\mathrm{z}} \boldsymbol{G}\right),
\end{aligned}
$$

the source terms are

$$
\overline{\mathbf{S}}=\frac{1}{\mathrm{~J}} \mathbf{S},
$$

and where

$$
\mathbf{Q}=\left[\begin{array}{c}
\rho \\
\rho \mathrm{u} \\
\rho \mathrm{v} \\
\rho \mathrm{w} \\
\rho \mathrm{pe}
\end{array}\right], \boldsymbol{E}=\left[\begin{array}{c}
\rho \mathrm{u} \\
\rho \mathrm{u}^{2}+\mathrm{P} \\
\rho u v \\
\rho \mathrm{uw} \\
(\rho \mathrm{e}+\mathrm{P})_{\mathrm{u}}
\end{array}\right], \boldsymbol{F}=\left[\begin{array}{c}
\rho \mathrm{v} \\
\rho v \mathrm{u} \\
\rho \mathrm{v}^{2}+\mathrm{P} \\
\rho v w \\
(\rho \mathrm{e}+\mathrm{P})_{\mathrm{v}}
\end{array}\right], \boldsymbol{G}=\left[\begin{array}{c}
\rho \mathrm{w} \\
\rho w u \\
\rho w v \\
\rho w^{2}+\mathrm{P} \\
(\rho \mathrm{e}+\mathrm{P})_{\mathrm{w}}
\end{array}\right], \mathbf{S}=\left[\begin{array}{c}
\mathrm{S}_{\mathrm{m}} \\
\mathrm{S}_{\mathrm{Fx}} \\
\mathrm{S}_{\mathrm{Fy}} \\
\mathrm{S}_{\mathrm{Fz}} \\
\mathrm{S}_{\mathrm{sw}}
\end{array}\right]
$$

The source terms are: (1) bleed per volume, (2) forces in the $\mathrm{x}, \mathrm{y}$, and $\mathrm{z}$ direction per volume, and (3) rate of shaft work per volume. The source terms are on a per unit volume basis because they are divided by the geometric Jacobian, $\mathrm{J}$, in the solution procedure, where 
$\mathrm{J}=\left[\begin{array}{lll}\frac{\partial \mathrm{x}}{\partial \xi} & \frac{\partial \mathrm{x}}{\partial \eta} & \frac{\partial \mathrm{x}}{\partial \zeta} \\ \frac{\partial \mathrm{y}}{\partial \xi} & \frac{\partial \mathrm{y}}{\partial \eta} & \frac{\partial \mathrm{y}}{\partial \zeta} \\ \frac{\partial \mathrm{z}}{\partial \xi} & \frac{\partial \mathrm{z}}{\partial \eta} & \frac{\partial \mathrm{z}}{\partial \zeta}\end{array}\right]$

These volumetric source terms are non-dimensionalized, in order to be consistent with the Euler equations, and presented as:

rate of mass bleed,

$S_{\dot{m}}=\frac{\left(\frac{\dot{m}}{\forall}\right)}{\rho_{\text {ref }} a_{\text {ref }}}$,

$\mathrm{x}$-force, $\quad \mathrm{y}$-force, $\quad \mathrm{z}$-force,

$S_{\mathrm{Fx}}=\frac{\left(\frac{F_{\mathrm{x}}}{\forall}\right)}{\rho_{\text {ref }} \mathrm{a}_{\text {ref }}^{2}}, \quad S_{\mathrm{Fy}}=\frac{\left(\frac{F_{\mathrm{y}}}{\forall}\right)}{\rho_{\text {ref }} \mathrm{a}_{\text {ref }}^{2}}, \quad S_{\mathrm{Fz}}=\frac{\left(\frac{F_{z}}{\forall}\right)}{\rho_{\text {ref }} \mathrm{a}_{\text {ref }}^{2}}$,

and rate of shaft work,

$S_{s w}=\frac{\left(\frac{\dot{s w}}{\forall}\right)}{\rho_{\text {ref }} a_{\text {ref }}^{3}}$. 
The reference quantities are based upon air $\left(R_{\text {air }}=1715.87 \frac{\mathrm{ft}-\mathrm{lb}}{\mathrm{slug} R}\right)$ at standard sea level conditions $\left(\mathrm{P}_{\mathrm{ref}}=14.696 * 144.0 \frac{\mathrm{lb}}{\mathrm{ft}^{2}}\right.$ and $\left.\mathrm{T}_{\mathrm{ref}}=518.67 \mathrm{R}\right)$ where

$$
\begin{aligned}
& \rho_{\text {ref }}=\frac{P_{\text {ref }}}{R_{\text {air }} T_{\text {ref }}}, \\
& a_{\text {ref }}=\sqrt{\gamma \mathrm{R}_{\text {air }} T_{\text {ref }}} .
\end{aligned}
$$

For closure, the ideal gas equation of state with a calorically perfect gas assumption follows in non-dimensional form:

$$
\begin{aligned}
& \mathrm{P}=\frac{\rho \mathrm{T}}{\gamma}, \\
& \mathrm{e}=\frac{\mathrm{T}}{\gamma}(\gamma-1)+\rho\left(\mathrm{u}^{2}+\mathrm{v}^{2}+\mathrm{w}^{2}\right) .
\end{aligned}
$$

The above equations of state allow non-dimensional pressure and temperature to be written in terms of conservation variables,

$$
\begin{aligned}
& \mathrm{P}=(\gamma-1) p\left(e-\frac{1}{2}\left(\mathrm{u}^{2}+\mathrm{v}^{2}+\mathrm{w}^{2}\right)\right), \\
& \mathrm{T}=\gamma(\gamma-1)\left(e-\frac{1}{2}\left(\mathrm{u}^{2}+\mathrm{v}^{2}+\mathrm{w}^{2}\right)\right) .
\end{aligned}
$$

When it is assumed that the turbomachinery source terms are supplied by an external mechanism, the equations presented above can be solved together to resolve the flow field on a fixed grid. 


\subsection{Turbomachinery Source Terms}

The turbomachinery source terms were calculated by modifying a streamline curvature code (SLCC) developed by Hearsey (1970). The SLCC technique has been well documented in the references; therefore, only a brief overview will be provided. The modification of the SLCC necessary for TEACC development will be presented. The modified SLCC will produce streamlines through the bladed region which will contribute to the development of control volumes. A control volume analysis will then be presented through the bladed region for the calculation of turbomachinery source terms.

\subsubsection{SLCC Technique}

The SLCC is based on a radial redistribution of blade force and shaft work producing an "axisymmetric flow with swirl" in the form of streamlines. Necessary inputs include overall geometry, blade geometry, and loss and deviation correlations. These correlations are obtained from experimental data. A typical grid, illustrated in Figure 3.2, extends far upstream and far downstream of the compressor. Axial stations are chosen to include the inlet and exit of the blades and other convenient stations to allow proper resolution of the flow field. Boundary conditions are required around the domain, with the outer casing and inner center body treated as streamlines. The boundary conditions are inlet total pressure, inlet total temperature, and inlet swirl angle with curvature equal to zero specified at both the inlet and exit. A particular operating point is established by specifying the rotor speed and overall mass flow. 
The radial momentum equation was developed from the Euler equations by assuming that entropy and enthalpy do not change along streamlines except as prescribed across the blades by the correlations. The conservation equations are simplified by replacing the axial momentum and the energy equation with these two assumptions. The remaining two equations, continuity and radial momentum, are solved simultaneously at each axial station. Two excellent references for the derivation of the streamline curvature method can be found in Hearsey (1970) and Novack (1967). A brief overview of the governing equations and assumptions is presented in Appendix C.

\subsubsection{Modifications of the SLCC Technique}

For the TEACC simulation to be highly responsive and capable of modeling transients, the SLCC must be restricted to a small axial region on either side of the blades. Since the SLCC is a subsonic flow solver, it requites a full set of boundary conditions around its domain. The SLCC boundary conditions of inlet and exit curvature, overall mass flow, swirl angle, total temperature, and total pressure are calculated from the TEACC flow field. Figure 3.3 is included to show the truncated grid in which the

SLCC operates. Although the boundary conditions are of the same type in this new mode of operation as they were before the grid was truncated to the blades, they are now more complicated since they are no longer uniform but a function of the time-dependent TEACC flow field around the blades.

The modifications made to the SLCC methodology are:

- Inlet and exit curvature 
- Overall mass flow and swirl angle

- Inlet total temperature and inlet total pressure

\subsubsection{Inlet and Exit Curvature}

At a given time, the 3D NPARC integrator provides a transient solution of the dependent variables which completely defines a spatial velocity field. Streamlines could be developed using the $3 \mathrm{D}$ velocity components. However, the SLCC requires curvature of the streamlines in only an axial-radial plane. Therefore, the curvature calculations provided by the $3 \mathrm{D}$ integrator are calculated from a circumferential projection onto a circumferential slice (axial-radial plane). This is effectively the same as calculating streamline curvature from only the axial and radial velocity components of the $3 \mathrm{D}$ transient velocity flow field. The streamline can be represented as a time-varying vector. Using the axial and radial plane as the projected plane, the in-plane projection can be written as

$\mathbf{r}=\left(\mathrm{x}, \sqrt{\mathrm{y}^{2}+\mathrm{z}^{2}}, 0\right)$

The in-plane curvature, $\mathrm{k}$, can be calculated from

$k^{2}=\frac{|\dot{r} \times \ddot{r}|^{2}}{\left(|\dot{r}|^{2}\right)^{3}}$

where the in-plane velocity vector is

$$
\dot{\mathrm{r}}=\frac{\mathrm{dr}}{\mathrm{dt}}=\left(\mathrm{u}, \sqrt{\mathrm{v}^{2}+\mathrm{w}^{2}}, 0\right)
$$


and the in-plane acceleration vector is

$$
\ddot{\mathbf{r}}=\frac{d \dot{\mathbf{r}}}{d t}=\left(\frac{d u}{d t}, \frac{d v}{d t}, 0\right)=\left(u \frac{d u}{d x}+v \frac{d u}{d y}, u \frac{d v}{d x}+v \frac{d v}{d y}, 0\right) .
$$

Curvature is a function of the local velocity and acceleration; therefore, it is very sensitive to the local numerical fluctuations. A strong discontinuity in sources to represent turbomachinery may cause the local flow field on either side of the blade row to experience numerical wiggles. Since curvature is a function of velocity gradients its calculation may be influenced by local velocity wiggles. The effects of the numerical oscillations in the velocity field were eliminated by using a least-squares approximation for the velocity field at the point where curvature calculations are made. This approximation is quadratic in both the axial and radial direction, thus bi-quadratic. Thirty-six adjacent grid points are used to determine the least-squares approximation. The decision to use 36 points and a nine-parameter bi-quadratic was a compromise between the desirable effect of smoothing out the numerical error and the undesirable effect of introducing a non-local bias.

\subsubsection{Overall Mass Flow and Swirl Angle}

Since TEACC uses the SLCC at each circumferential and radial control volume, individual mass flows were calculated and summed together to get the total mass flow within a segment. The local mass flow within a radial control volume, $\mathrm{g}$, is

$$
\dot{\mathrm{m}}_{\mathrm{g}}=(\rho \mathrm{V} \cdot \mathbf{A})=(\rho \mathrm{u}) \mathrm{A}_{\mathrm{x}, \mathrm{I}}+(\rho \mathrm{v}) \mathrm{A}_{\mathrm{y}, \mathrm{I}}+(\rho \mathrm{w}) \mathrm{A}_{z, \mathrm{I}}
$$


The mass flow within a circumferential segment is obtained by summing the local radial mass flows within a segment by

$$
\dot{\mathrm{m}}_{\text {Segment }}=\sum_{\mathrm{g}=1, \mathrm{~K}} \dot{\mathrm{m}}_{\mathrm{g}}
$$

where $\mathrm{k}$ is the number of control volumes within a segment. The overall compressor mass flow was obtained by summing the total mass flow within each circumferential segment for the total number of segments.

A radial distribution of swirl angle is calculated at the inlet of each control volume on a circumferential segment. Swirl angle is calculated at the inlet face of each radial control volume, $\mathrm{g}$, and defined as the arc-tangent of tangential velocity divided by axial velocity, which is

$$
\alpha_{g}=\tan ^{-1} \frac{\left(V_{\theta}\right)}{(\mathrm{u})}\left(\frac{180}{\pi}\right)
$$

where the units are converted from radians to degrees and

$$
\left(\mathrm{V}_{\theta}\right)=-(\mathrm{v}) \sin \theta+(\mathrm{w}) \cos \theta
$$

\subsubsection{Inlet Total Temperature and Inlet Total Pressure}

The total temperature and total pressure are calculated by using the conservation variables, ideal gas relations, and the compressible form of the stagnation definitions. At the point where the totals are desired, the total temperature is

$$
T_{t}=\left\{T+\frac{1}{2}(\gamma-1)\left(u^{2}+v^{2}+w^{2}\right)\right\} T_{\text {ref }}
$$


and the total pressure is

$$
P_{t}=P\left(\frac{T_{t}}{T}\right)^{\frac{\gamma}{\gamma-1}}\left(\gamma P_{\text {ref }}\right)
$$

With the boundary conditions for the SLCC calculated from NPARC, an axisymmetric solution can be obtained at each time step.

\subsubsection{Technique to Calculate Turbomachinery Source Terms}

The source term calculations are performed after the SLCC converges to a steadystate solution through the bladed region. The technique for calculating turbomachinery source terms uses control volumes within the bladed region and applies steady-state conservation laws across each volume. Since the conservation of angular momentum is maintained in the axisymmetric solution of the SLCC, a radial distribution of circumferential velocity vectors is produced. This can be referred to as an "axisymmetric flow with swirl" solution. Control volumes are constructed through the bladed region with steady-state velocities and pressures known on all surfaces from streamline calculations. The forces in the axial, radial, and circumferential directions on these control volumes and the rate of shaft work can then be obtained.

\subsubsection{Equations Used for Sources}

From an Eulerian perspective, a control volume with finite area and volume has been constructed from streamlines with steady-state fluid passing through it. Cartesian control volumes are constructed over a circumferential segment of the bladed region using streamlines and overall blade geometry. Details of the control volume 
construction, along with surface area and volume calculations, are detailed in Appendix D. The conservation equations used to calculate turbomachinery source terms over fixed control volumes are:

mass bleed,

$$
\dot{\mathrm{m}}_{\mathrm{b}}=\sum\left(\dot{\mathrm{m}}_{\mathrm{b}}\right)_{\mathrm{h}}=\frac{\partial}{\partial \mathrm{t}} \int_{\mathrm{cv}} \rho \mathrm{d} \forall+\int_{\mathrm{CS}} \rho \mathbf{V} \bullet \mathrm{d} \mathbf{A}
$$

forces,

$$
\mathbf{F}=\sum(\mathbf{F})_{\mathrm{h}}=\frac{\mathrm{d}(\mathrm{m} \mathbf{V}))}{\mathrm{dt}}=\frac{\partial}{\partial \mathrm{t}} \int_{\mathrm{cV}} \rho \mathbf{V} \mathrm{d} \forall+\int_{\mathrm{cS}} \mathbf{V}_{\rho} \mathbf{V} \bullet \mathrm{d} \mathbf{A}
$$

and rate of shaft work,

$$
S \dot{W}=\sum(\operatorname{SW})_{h}=\frac{\partial}{\partial t} \int_{C V} \rho \operatorname{ed} \forall+\int_{C S}\left(\frac{P}{\rho}+e\right) \rho V \bullet d A
$$

It is important to interpret the control volume analysis results properly. If viewed from the continuity perspective, there are no bleeds currently modeled in the compressor simulation, but if bleeds were needed, all of them would be collected together with no detailed knowledge of each individual bleed. Likewise, we may view the sum of forces in the momentum equations as a collection of many forces without knowing the details of each separately. Specifically, only inertial forces and pressure area forces (Figure 3.4) have been modeled on the RHS of Equation 3.29, allowing the sum of the forces to include viscous effects, boundary effects, shock effects, circumferential effects, and a resultant force to hold the blade in steady state. This gives us a summation of all the effects but no knowledge of individual external forces. If we wanted to isolate a force 
such as circumferential effects, this effect would be modeled on the RHS of Equation 3.29 , and the sum of the forces would be adjusted by that amount. Finally, we will focus on the sum of the energy passing through the control volume. An overarching assumption is that there is no heat transfer in the compressor simulation, and the work has been broken into shaft work and flow work. The flow work has already been included on the RHS of Equation 3.30, meaning that the sum of the energy is the sum of the rate of shaft work. Three forms of energy are already accounted for in the control volume analysis: internal energy, kinetic energy, and flow work. The summation of the work collects everything into one term. Thus, the sums of the bleeds, forces, and rate of shaft work are the turbomachinery source terms used by the TEACC simulation for compressor modeling, and a further breakdown of the composition of the source terms is unnecessary. This observation is grounded in the fact that the Euler equations account for the convection and the pressure area terms. The source terms need to account for everything else in order to model the turbomachinery.

\subsubsection{Source Term Assumptions and Calculation Procedures}

This section will delineate the source term assumptions and expound on the previously outlined control volume analysis to calculate the external forces in the axial, radial, and circumferential directions, and the rate of shaft work. Since the streamlines pass through the center of the interior control volumes, axial, radial, and circumferential velocity, pressure, and density are known and assumed to be uniform across the primarily axial (inlet and exit) faces (Figure 3.4). For the control volume faces pointing primarily 
in the y-direction (top and bottom), pressure is calculated by the algebraic sum of the four pressures on the inlet and exit axial faces of the adjacent control volumes. For control volume faces pointing primarily in the z-direction (left and right), pressure is calculated as an average of the pressure on the axial faces associated with the streamline moving through the center of the control volume.

An assumption that the top and bottom surfaces of each control volume is a streamsurface is incorporated to simplify the analysis. Mass does not cross a streamsurface; neither does momentum or energy. Therefore, there is no need to calculate an average velocity operating on the top or bottom surface of the control volume. Because of symmetry, it is clear that momentum circumferentally entering the left face leaves the right face, producing no net momentum contribution or need to calculate an average velocity on these two faces.

One additional quantity, the local mass flow passing through the control volumes, must be calculated before the control volume analysis can be completed. Since mass does not cross a streamsurface, the total mass flow passing through the inlet and the exit of each SLCC control volume is equal, as given by the steady-state continuity equation. Due purely to convenience, as to when variables are stored, the mass flow was calculated on the exit faces, not the expected inlet, of the control volumes. The exit Cartesian area components and exit velocity vector components are combined with density as follows:

$$
\dot{\mathrm{m}}=\rho \mathbf{V} \cdot \mathbf{A}=\left(\mathrm{A}_{\mathrm{x}, \mathrm{E}} \mathrm{V}_{\mathrm{x}, \mathrm{E}}+\mathrm{A}_{y, \mathrm{E}} \mathrm{V}_{\mathrm{y}, \mathrm{E}}\right) \frac{\mathrm{P}_{\mathrm{E}}}{\mathrm{T}_{\mathrm{E}} R}
$$


where density is calculated from ideal gas assumptions.

The turbomachinery source terms can now be calculated using the above assumptions. As stated above, because of the streamsurface assumption and symmetry, only the inlet and exit faces contribute to the surface integrals for inertial forces. However, there is a potential pressure force contribution on each face for the calculation of forces. The sum of the axial forces is:

$$
F_{x}^{S L C C}=P_{E} A_{x, E}-P_{1} A_{x, I}-P_{B} A_{x, B}+P_{T} A_{x, T}+\dot{m}\left(V_{x, E}-V_{x, I}\right)
$$

The inlet, exit, top, and bottom surfaces each contribute a pressure area force in the axial direction, but the circumferential faces, due to their construction, do not contribute to an axial component of force. The sum of the forces in the y-direction is:

$$
F_{y}^{S L C C}=-P_{1} A_{y, I}+P_{E} A_{y, E}-P_{B} A_{y, B}+P_{T} A_{y, T}+2 * P_{R} A_{y, R}+\dot{m}\left(V_{y, E}-V_{y, I}\right)
$$

The inlet, exit, top, and bottom give an expected force contribution due to pressure and area. However, this is a Cartesian analysis over a very narrow segment, not a cylindrical analysis, and the circumferential faces of the grid give a pressure-area contribution to the sum of the forces in the y-direction. The sum of the forces in the $z$ direction is:

$$
\mathrm{F}_{\mathrm{z}}^{\mathrm{SLCC}}=\dot{\mathrm{m}}\left(\mathrm{V}_{\mathrm{z}, \mathrm{E}}-\mathrm{V}_{\mathrm{z}, \mathrm{l}}\right)
$$

Neither the inlet and exit faces nor the top and bottom streamsurfaces have a component of area in the z-direction. This means that the forces in the z-direction effectively receive only a component from the momentum calculations. Since the 
axisymmetric solution associated with the streamlines also delivers swirl, the velocity components in the z-direction provide the mechanism to calculate forces in this direction. The sum of the rate of work is:

$$
\dot{\mathrm{SW}}^{\mathrm{SLCC}}=\dot{\mathrm{m}} \mathrm{c}_{\mathrm{p}}\left(\mathrm{T}_{t, \mathrm{E}}-\mathrm{T}_{\mathrm{t}, \mathrm{I}}\right)
$$

Due to symmetry, there is no contribution of work on the left and right faces. No contribution is given for the top and bottom surfaces since no mass flow crosses these faces. The only contribution is from the inlet and exit faces.

Now that the turbomachinery source terms are calculated, they are divided by local volume to put them in the proper TEACC format. This approach is also very useful because the volumetric extent over which the sources were obtained has been removed. This means the volumetric sources can be easily scaled to TEACC with any circumferential extent (number of degrees between segments) by multiplying by the larger local volume. The source terms are then non-dimensionalized consistent with the Euler equations.

\subsection{Grid Structure Tailored for Turbomachinery}

To solve the governing conservation equations with a numerical solution technique, a structured grid with physical coordinates $(\mathrm{X}, \mathrm{Y}, \mathrm{Z})$ is necessary. The Cartesian grid is mapped by a coordinate transformation to a generalized curvilinear coordinate system represented by indices $(\mathrm{J}, \mathrm{K}, \mathrm{L})$ where each integer combination represents a physical grid node (intersection of grid lines). Both the physical coordinate system and the generalized curvilinear coordinate system are right-handed, rendering all 
volume calculations positive. TEACC uses this fixed grid to resolve the conservation equations on the nodes.

A cylindrical (O-type) grid is constructed through the internal flow path for the simulation of turbomachinery as presented in Figure 3.5a. In keeping with the above general guidelines, each physical grid point is specified by a right-handed Cartesian coordinate. The grid is constructed along the physical interior boundaries of the turbomachinery inner (center-body) and outer casing. Restrictions are placed on some of the grid lines, as can be seen in the axial-radial view of the grid in Figure 3.5b. Grid lines are restricted to map the leading and trailing edges of the bladed region. Also, the grid is packed through the bladed region to minimize numerical losses with strong gradients. The grid is not packed near the inner and outer casing since viscous effects on the walls are ignored; however, the grid should be located close enough to the walls to resolve the boundary conditions accurately without restricting the overall CFL stability criterion. The grid is wrapped in the circumferential direction and overlapped by exactly one circumferential segment to facilitate the wrap-around boundary condition explained in Section 3.4. Each circumferential segment is equally spaced and defined in the radialcircumferential view of the grid in Figure 3.5c.

The circumferential grid construction can be greatly simplified with clean inlet operation because the three-dimensional flow field is axisymmetric. Currently, TEACC does not have an "axisymmetric with swirl" capability, but this can be simulated with a minimum of five circumferential segments spanning only part of the circumferential 
direction. This mode is particularly valuable for clean inlet investigations because the solution is "axisymmetric flow with swirl" and the grid can be significantly refined in the circumferential direction without increasing the computational resource requirements per time step. Grid density and refinement as it relates to the solution will be developed in Chapter 6.

\subsection{TEACC Boundary Conditions}

The time-dependent Euler equations with source terms define a hyperbolic system of equations requiring a full set of boundary conditions specified at all boundaries for subsonic flow. Since many types of boundary conditions have been developed and made available with NPARC (Cooper, 1989), no new boundary condition routines were required. Each of the boundary conditions used is outlined below, while the details are presented in the NPARC documentation. All the boundary conditions used with NPARC are explicit.

The inflow boundary condition is based on reference plane characteristics, and the total pressure and total temperature at the inlet must be specified. Inlet flow directions are assumed to be normal to the boundary (Figure 3.6). The grid was constructed so that the inlet plane was perpendicular to the centerline; therefore, the inlet flow direction was specified to be parallel to the centerline. A final relationship needed to specify the inlet boundary condition completely comes from the compatibility equation constructed along the (u-a) characteristic which passes information from the interior algorithm to the inlet boundary condition. The compatibility equation, along with the definition for total 
temperature and total pressure is solved iteratively for static pressure, static temperature, and velocity. The primitive variables $\rho, \mathrm{u}, \mathrm{v}, \mathrm{w}$, and $\mathrm{p}$ are used to calculate the conservative variables.

The exit boundary condition does not make direct use of reference plane characteristics. A flow field with a strong swirl from rotating turbomachinery will have a strong exit static pressure profile. This is dealt with by specifying the static pressure at a single point on the exit. The shape of the static pressure profile one axial station upstream is imposed on the exit, which is scaled by the specified static pressure (Figure 3.7). Therefore, the exit static pressure shape is imposed effectively by the conservation equations. This is a particularly good approach when the last few axial stations maintain uniform diameter. The remaining primitive variables are obtained from a zeroth-order extrapolation and combined with static pressure to calculate the conservative variables.

The wall boundary conditions are assumed to be slip wall; the normal velocity components are set equal to zero at the solid walls (Figure 3.8). A zeroth-order extrapolation is used to calculate the remaining primitive variables from the adjacent interior nodes. Although no viscous effects are included, a grid structure should have a set of grid points close to the walls to resolve the wall boundary conditions accurately.

There are two types of boundary conditions used in the circumferential direction; they are: (1) rotationally periodic (wrap-around) and (2) rotationally cyclic. The rotationally periodic boundary condition is used when the grid is constructed over the full annulus. An O-type grid was used to facilitate the use of this boundary condition 
where the circumferential seam of the grid was overlapped by one circumferential segment (Figure 3.9); thus, the five conservation variables represented below by $\varphi$ at either end of the seam were defined by:

node 1

$\varphi_{1}=\varphi_{\mathrm{L}-1}$ $\underline{\text { node } \mathrm{L}}$

$$
\varphi_{\mathrm{L}}=\varphi_{2}
$$

The rotationally cyclic boundary condition is used with a clean inlet where the solution is expected to be "axisymmetric with swirl." An O-type grid is still used, but the grid spans only part of the circumferential direction. In Figure 3.10 a grid with four circumferential segments is constructed to illustrate this boundary condition. The conservative variables at node 2 are mapped to node $L$, and the conservative variables at node ( $\mathrm{L}-1)$ are mapped to node 1 by the following procedure:

$\underline{\text { node } 1}$

$\underline{\text { node L }}$

$\rho_{1}=\rho_{L-1}$

$$
\rho_{L}=\rho_{2}
$$

$\rho u_{1}=\rho u_{L-1}$

$\rho \mathrm{u}_{\mathrm{L}}=\rho \mathrm{u}_{2}$

$e_{1}=e_{L-1}$

$\mathrm{e}_{\mathrm{L}}=\mathrm{e}_{2}$

$\rho \mathrm{v}_{1}=\rho \mathrm{v}_{\mathrm{L}-1} \cos \theta-\rho \mathrm{w}_{\mathrm{L}-1} \sin \theta$

$\rho v_{L}=\rho v_{2} \cos \theta-\rho w_{2} \sin \theta$

$\rho \mathrm{w}_{1}=\rho \mathrm{v}_{\mathrm{L}-1} \sin \theta+\rho \mathrm{w}_{\mathrm{L}-1} \cos \theta$

$\rho \mathrm{w}_{\mathrm{L}}=\rho \mathrm{v}_{2} \sin \theta+\rho \mathrm{w}_{2} \cos \theta$

\subsection{Initializing the TEACC Flow Field with SLCC}

Because TEACC has been formulated with a time-dependent solution technique, the flow field must be initialized with a set of initial conditions. A point on the compressor map was chosen so that all calculations remain physical. The SLCC is used 
for this initialization of the flow field by operating on a grid which extends to the boundaries of the 3D fixed grid used by NPARC. The inlet and exit are located far upstream and far downstream of the compressor where curvature specified as zero is a good boundary condition. The inlet swirl velocity is specified to be zero, and the inlet total pressure and inlet total temperature are specified to be constant at 14.697 psia and 518.67 R, respectively. Finally, the overall mass flow and the percent of design corrected speed must also be specified. The SLCC, along with these specified boundary conditions, provides a solution of streamlines which can be transformed into the conservation variables needed by NPARC. These conservation variables are then interpolated from the SLCC streamline grid to the NPARC fixed grid. The flow field is assumed to start with a clean inlet so the solution is distributed to each circumferential segment of the flow field.

\subsection{Convergence Criteria}

TEACC as well as NPARC uses the time-stepping method to achieve steady-state. There are two criteria used to verify TEACC's convergence to steady-state. First, the L2 norm of the residual is dropped to machine zero or at least four orders of magnitude. The L2 norm of the residual vector is:

$\|$ Residual $\|_{2}=\sqrt{\left(\sum_{q=1, n} \text { Residual }\left.\right|_{q} ^{2}\right)} ; \mathrm{n}=$ number of nodes, 
where the components of the residual vector at each node are the residuals of each conservation equation. A residual is defined as the explicit portion of a discretized conservation equation which is driven to zero at steady-state.

The second criterion to verify TEACC's convergence to steady-state was to monitor key variables of interest and determine that they no longer vary as TEACC continues to iterate. The key variables used for turbomachinery convergence were the integrated mass flow rates of segments bisecting the four quadrants around the grid circumference.

It was observed, in general, that dropping the L2 norm of the residual by four orders of magnitude was very close to machine zero and usually brought the flow field to steady-state. However, near the stalled region of the turbomachinery simulation, TEACC dropped the L2 norm of the residuals by four orders of magnitude but behaved as in a slow transient. Hence, iterations continued until both convergence criteria were reached.

\subsection{TEACC Solution Procedure}

Both TEACC and NPARC solve the governing equations with the Beam and Warming approximate factorization algorithm (Beam, 1976) to obtain a flow-field solution. This solution procedure incorporated by TEACC to solve the time-dependent Euler equations is widely used in the engineering community. As the grid is refined the approximate factorization algorithm approximates a solution to the Euler equations (Beam, 1976). A discretized solution to the Euler equations is then compared to experimental data to determine the accuracy of the TEACC simulation. 
The above equations and metrics (Equations 3.1-3.17) associated with curvilinear coordinates are consistently discretized with central differences to produce an implicit algorithm. The governing equations were cast in strong conservation form to keep global conservation, even in the presence of strong shocks. The technique of Pulliam and Steger (1980) to implement the Beam and Warming implicit algorithm was incorporated to factor the coefficient matrices, requiring a penta-diagonal solver. Since this is a finite difference algorithm, artificial dissipation is required for numerical stability. NPARC uses the Jameson-style (1981) improved shock capturing artificial dissipationThe secondorder dissipation was set to zero and the forth-order dissipation was set to 0.60 for all turbomachinery simulations. A flow chart outlining TEACC's solution procedure is presented in Figure 3.11.

- The development of TEACC as a turbomachinery simulation requires the inclusion of proper mathematics to simulate turbomachinery physics and a solution procedure which converges to the true mathematical solution. The turbomachinery is calculated by a SLCC and included in the simulation by source terms. The detailed mathematical issues associated with residual convergence, the discrete problem solution convergence, and convergence to the true mathematical solution are discussed in the derivation of the Beam and Warming solution technique derivation. TEACC's accuracy to the true converged solution is further enhanced by comparison to experimental data

The inclusion of semi-actuator disc source terms is new to NPARC and has been included to model the effects of turbomachinery. The source terms were imposed 
explicitly in NPARC on the right-hand side (RHS) of the discretized governing equations. To include the source terms implicitly would have required the calculation of a source Jacobian. The development of the source Jacobian would have required five solutions from the SLCC instead of the current single solution to develop turbomachinery source terms. The SLCC is already computationally time-consuming, and the potential benefit gained by making the source terms implicit was judged not to be cost effective in this initial turbomachinery investigation. Inclusion of the source Jacobian on the LHS may affect the transient behavior of the solution, but the steady-state solution will remain unaffected. The other potential benefit of including the source Jacobian on the LHS would be to improve stability. However, with the implicit Euler equations and the explicit source terms, TEACC maintained CFL's in the 5 to 10 range for all turbomachinery applications and gave robust solutions with comparable convergence rates to that of inviscid NPARC duct flow solutions on the same grids, the source terms were left explicit. 


\subsection{Methodology to Implement Source Terms into TEACC}

With the TEACC-supplied boundary conditions, the SLCC produces an axisymmetric solution through the bladed region in the form of streamsurfaces which can be constructed into control volumes to calculate turbomachinery source terms. This section's focus is to show how the SLCC sources are implemented into TEACC. The technique for distributing the sources radially and circumferentially using the SLCC will be described. The sources will, through the use of a weighting function, be distributed axially through the grid-packed bladed region. Although this distribution of sources generally provided stable operation, choked flow calculations were unstable and required source modifications. Among the several source modification techniques investigated, one proved to be stable and robust. Finally, the techniques for distributing and modifying the turbomachinery source terms were algebraically combined for implementation into TEACC.

\subsection{Radial Interpolation of Turbomachinery Source Terms}

Source terms are calculated by the SLCC, using a grid that changes as flow conditions change due to the recalculation of streamlines. To implement these source terms into TEACC, a radial interpolation technique was derived. A radial distribution of sources was constructed by selecting the radius at the center of each SLCC source control volume (Figure 4.1a). This radius was nondimensionalized by the radial extent of the inner and outer casing in the axial center of the blade, and a radial distribution of nondimensional turbomachinery sources was constructed as shown in Figure $4.1 \mathrm{~b}$. An 
Akima spline (Akima, 1970) was used to interpolate these source terms to the TEACC grid along a single circumferential segment of the bladed region. A radial distribution of TEACC control volumes was defined through the bladed region using the existing grid structure as shown in Figure 4.1c. Each radial control volume is defined by the axial extent of the bladed region and the boundaries of the circumferential extent. The radius associated with the center of each of these radial control volumes defined a radial distribution of TEACC volumes through the bladed region of a circumferential segment. The radius was non-dimensionalized by the radial extent of the inner and outer casing in the middle of the bladed region. A radial distribution of TEACC source terms was acquired by interpolating the fixed TEACC volume centers onto the function of sources developed earlier from the SLCC as shown in Figure 4.1d.

\subsection{Circumferential Distribution of Turbomachinery Source Terms}

A technique which is sensitive to the local flow field in the circumferential direction must also be implemented to obtain a circumferential distribution of turbomachinery source terms. This is especially true for the modeling of circumferential distortion where the inlet total pressure may vary greatly in the circumferential direction. For a distorted inlet upstream of the compressor, a strong circumferential inlet distortion was imposed on the system, as in Figure 4.2a. This distortion affected the adjacent flow field, and Figure 4.2b shows that this circumferential distortion, ignoring viscous effects, persisted up to the compressor inlet (Longley,1992). The compressor's performance was greatly affected by this distortion, causing a very nonuniform circumferential flow field. 
The modeling technique for acquiring a circumferential distribution of turbomachinery source terms applied the SLCC separately at each circumferential segment, as shown in Figure 4.2c. The SLCC is very sensitive to the changing demands of the flow field because it acquires its boundary conditions immediately upstream and downstream of each circumferential segment. Therefore, the SLCC interpolates a new radial distribution of turbomachinery source terms sensitive to the changing flow field for each circumferential segment, as shown in Figure 4.2d. With a more complex inlet distortion pattern, the number of circumferential segments would be increased to maintain the high fidelity of the TEACC simulation. Together, this section and the previous section present how the SLCC is used to radially and circumferentially distribute the sources to the TEACC simulation. The following section shows how the sources are distributed axially through the packed bladed region.

\subsection{Axial Distribution of Turbomachinery Source Terms}

TEACC packs the grid through the bladed region to reduce the numerical error from strong flow gradients. In general the axial work and force distribution is unknown through the bladed region; therefore, a great deal of freedom exists in how the sources can be distributed through the bladed region. The sources are distributed conservatively through the bladed region by requiring that the sum of the sources distributed through the NPARC grid equal the sum of the sources developed in the SLCC. A simple technique to investigate the axial distribution of sources was incorporated by using a distribution function which could take on a variety of linear shapes (Figure 4.3a). The distribution 
function ranged over the axial nodes of the bladed region. Its shape was specified by integer magnitudes varying from zero to eight, with zero providing no sources to a node and eight providing full sources to a node. To facilitate the construction of the distribution function, a percentage of dwell could be specified in the middle of the blade row, allowing the distribution to ramp toward the middle of the blade with a plateau of uniform sources in the interior. The distribution functions were designed to be able to slowly bring on the impact of the strong sources to shift the emphasis toward the center of the blade. This approach avoids the sudden impact of strong sources which could produce unnecessary oscillations, causing loss of accuracy and robustness in the turbomachinery simulation.

The weighting function through the bladed region was constructed only from local volumes through the bladed region and the distribution function. Because the volume varies for each axial slice through the bladed region, a radial distribution of weighting functions was produced. The weighting function can be calculated from

$$
\omega_{\mathrm{JK}}=\delta_{\mathrm{K}} \mathrm{d}_{\mathrm{J}}
$$

where $d_{J}$ is the axial distribution function and the radial distribution, $\delta_{K}$, is defined by

$$
\delta_{K}=\frac{\forall_{K}}{\left(\sum_{i=1, J} d_{i} \forall_{i}\right)_{K}}
$$

where $\forall_{K}$ is the sum of the local axial volumes at a given axial slice and $\forall_{i}$ is a local volume in an axial slice. 
The flexibility in the distribution function was initially included as a fine-tuning control to optimize the solution for convergence. It was expected that the distribution function, which sloped up to the middle with a plateau spanning the middle part of the blade, would give the best results since the transition from no sources to strong sources would take place slowly. This was not the case. A variety of distribution shapes incorporating this plateau theory were evaluated. It was found that the overall solution did not change noticeably for a distribution function that allowed convergence. Therefore, the choice of appropriate distribution function was based upon stability and robustness. The distribution functions which ramped continuously across the blade, reaching a maximum value at the leading or trailing edge of the blade, failed to give converged solutions. The distribution functions which ramped toward the center with or without a dwell in the middle produced converged solutions, but were restricted in robustness. Robustness was measured by how much the exit static pressure could be moved to calculate a new steady-state point and remain stable. A uniform distribution function was found to be the most robust (Figure 4.3b). Two factors were observed to give the best results: (1) keep the relative change in sources through the blade at a minimum, and (2) keep the maximum value in the bladed region also at a minimum. This means that robustness increases by increasing the number of axial control volumes within the bladed region; however, this must be balanced by memory constraints and minimization of high aspect-ratio control volumes. 


\subsection{Source Modifications Necessary for Stability}

The TEACC turbomachinery simulation with a clean inlet was observed to remain stable at low corrected speeds $(50 \%$ and $70 \%)$, but was unstable in the choked regions (mass flow restricted at the minimum area where Mach number $=1.0$ ) of the high corrected speed ( $90 \%$ and $100 \%$ ) simulations. Several techniques were tried to regain stability in the region of clean inlet operation. Some of the techniques used to improve TEACC's stability are presented below.

The first attempt to regain stability was to arbitrarily widen the corrected mass flow range in the choked region to lessen the rate of change of loss and exit relative blade angle (defined in Appendix C) with mass flow. This approach seemed appropriate, based upon 1D experience where steep characteristics had caused stability problems. Unfortunately, the loss and exit relative blade angle characteristics could not be widened enough to affect the solution without losing all physical significance. This approach was abandoned.

Another approach to regain stability was based upon a relaxation factor. A fixed relaxation factor was placed on the inlet total mass flow supplied to the SLCC as a boundary condition from the TEACC simulation at each time step. The form of the relaxation technique along with the range of usable values is given by:

$$
\dot{\mathrm{m}}^{\text {Affer }}=\dot{\mathrm{m}}^{\mathrm{p}}+\phi\left(\dot{\mathrm{m}}^{\text {Before }}-\dot{\mathrm{m}}^{\mathrm{p}}\right) ; \quad .001 \leq \phi \leq .01,
$$


where $\dot{\mathbf{m}}^{\mathbf{P}}$ is the rate of mass flow at the previous time step and $\phi$ is the relaxation factor. This brought stability over a wider range of corrected mass flow. The TEACC simulation gave the correct answer when the solution converged, but its stability was fragile. This expanded the range into choked flow, but convergence rates were low compared to the regions where relaxation factors were not needed. A natural follow-on was to put a relaxation factor on an exit quantity for the SLCC instead of overall mass flow.

A fixed relaxation factor of the same form as presented earlier was placed on the sources interpolated from the SLCC as follows:

$$
\mathrm{S}^{\text {After }}=\mathrm{S}^{\mathrm{p}}+\phi\left(\mathrm{S}^{\text {Before }}-\mathrm{S}^{\mathrm{p}}\right) ; \quad .001 \leq \phi \leq .01,
$$

where $\mathbf{S}^{\mathbf{P}}$ is each of the turbomachinery source terms at the previous time step and $\phi$ is the relaxation factor. This relaxation factor was applied before the sources were distributed axially through the packed grid. For solutions where convergence was obtained, the TEACC simulation consistently converged to the same reasonable solutions. This technique greatly increased the range of stability and brought convergence rates comparable to those observed in the non-choked regions. It gave the best simulation to this point, but it was limited in the area of robustness, which was measured by determining how far the exit backpressure could be moved without the solution becoming unstable.

In keeping with the improvement found from using the fixed relaxation factor on the sources, a variable relaxation factor was applied. This meant a different relaxation factor was applied axially to the sources in the packed grid of the bladed region. This 
technique delivered the correct solution, established robustness which allowed the exit static pressure to be moved for operation at adjacent flow points, and provided the best convergence rates for any of the techniques investigated.

The variable relaxation factor investigated was a geometric progression which changed the relaxation factor through the bladed region, as shown in Table 4.1. In general, no relaxation factors were needed in regions of the flow field away from the compressor choke point with a clean inlet. In the choked region, an initial $\phi=0.7$ gave the best results. With a strong initial relaxation factor $(\phi<0.5)$ the solution required a longer time period to satisfy both parts of the convergence criteria (L2 norm drops four orders of magnitude and local variables do not vary with time) to reach steady-state. The back of the bladed region had such a strong relaxation factor that the sources continued to change slowly, placing the simulation in a very slow transient. In this situation, convergence to a steady-state solution was established when the overall mass flow within each segment of the simulation no longer varied in the seventh significant figure.

The relaxation factor was required for inlet distortion simulation. Usually $\phi=0.7$ was sufficient to maintain robustness and good convergence. When calculating distortion, TEACC is forced to function in the two most unstable places simultaneously. The clean inlet part of the compressor is operating in the choked or near-choked part of the compressor while the distorted part of the compressor is operating near the stalled part of the compressor. However, with the variable relaxation factor, the TEACC simulation remained stable and produced converged solutions with clean and distorted inlets. 


\subsection{Mathematical Representation of Source Term Implementation}

Many pieces of the simulation were developed earlier in this chapter. This section implements the information developed in the current chapter into a single technique to calculate properly distributed turbomachinery sources within the bladed region. The calculations described above are presented in algebraic form to emphasize their meaning and to show how some of the source terms are treated differently. The source terms for a given bladed TEACC control volume are:

$$
\begin{aligned}
& F_{x, \text { JKL }}=\omega_{J K}\left(\left[\frac{F_{x}}{\forall}\right]^{\text {SLCC }}\right)_{\mathrm{KL}}^{\text {Relax }} \forall_{J K L} \\
& F_{y, J K L}=\omega_{J K}\left[\left(\left[\frac{F_{y}}{\forall}\right]^{\text {SLCC }}\right)_{\mathrm{KL}}^{\text {Relax }} \cos \theta_{L}-\left(\left[\frac{F_{z}}{\forall}\right]^{\text {SLCC }}\right)_{K L}^{\text {Relax }} \sin \theta_{L}\right] \forall_{J K L} \\
& F_{z, \text { JKL }}=\omega_{\text {JK }}\left[\left(\left[\frac{F_{y}}{\forall}\right]^{\text {SLCC }}\right)_{\mathrm{KL}}^{\text {Relax }} \sin \theta_{\mathrm{L}}+\left(\left[\frac{F_{z}}{\forall}\right]^{\text {SLCC }}\right)_{\mathrm{KL}}^{\text {Relax }} \cos \theta_{\mathrm{L}}\right] \forall_{\mathrm{JKL}} \\
& \operatorname{SW}_{\mathrm{JKL}}=\omega_{\mathrm{JK}}\left(\left[\frac{\mathrm{s} \dot{W}}{\forall}\right]^{\mathrm{SLCC}}\right)_{\mathrm{KL}}^{\mathrm{Relax}} \forall_{\mathrm{JKL}}
\end{aligned}
$$

where,

(1) sources were distributed radially, based upon the radial distribution of the sources calculated in the SLCC, 
(2) a transformation (trigonometric relations) was performed on the circumferential forces per volume calculated from the SLCC to distribute them to the appropriate circumferential segment in Cartesian coordinates,

(3) a variable relaxation factor (superscript Relax) was applied to the interpolated sources,

(4) a weighting function, $\omega_{\mathrm{JK}}$, was based upon a specified distribution function and the local volume distribution through the axial extent of the bladed region, and

(5) volume, $\forall_{\mathrm{JKL}}$, was calculated from the local NPARC grid structure. 


\subsection{Experimental Data for Comparison with TEACC}

Rotor 1B (Seyler and Gestolow, 1967) was chosen as the TEACC simulation vehicle because it offered simplicity in the number of stages ( 1 blade row), but represented a compression system with a thorough analysis of clean and distorted inlet. Rotor 1B is a high- performance rotor similar to those found in modern high-speed aircraft. Information conveyed about Rotor 1B in this chapter is presented to enhance the compressor modeling technique, and to familiarize the reader with the experimental data used for comparison with TEACC.

\subsection{Characteristics of the NASA Rotor 1B}

NASA Rotor 1B (Seyler and Gestolow, 1967) was designed by NASA in 1967 as a transonic single compressor blade row without an inlet or exit guide vane to alter the flow field. Rotor 1B was designed to advance the state of the art in compressor theory by increasing blade speed and stage loading while, at the same time, maintaining a high efficiency with a large practical stall margin. During this time, a workable titanium alloy was developed which made it possible for the 36.5-in.-diameter rotor to produce a rotor tip speed of 1,400 feet per second. The rotor was further designed with a multiple circular-arc blade shape which was applied over the top $40 \%$ of the blade while a double circular-arc construction was employed for the bottom $60 \%$ of the blade. With a hub-totip ratio at the rotor inlet of 0.5 , the blade sections were long enough to require a midspan damper to maintain structural integrity during operation. Rotor 1B consisted of 44 blades, producing a moderate solidity of 1.3 at the rotor tip. Rotor 1B produced a design 
corrected mass flow of $215.49 \mathrm{lb} / \mathrm{sec}$, a total pressure ratio of 1.6, and had an efficiency of 0.858 on the 100 -percent corrected speed line.

\subsection{Test Facility}

Rotor 1B was tested at General Electric's Compressor Test Facility at Lynn, Massachusetts (Figure 5.1). The test rotor drew air from the atmosphere through two banks of filters. The first filter bank was designed to remove 22-percent of the particles larger than three to five microns. The second filter bank was designed to remove 90-95 percent of the remaining particles down to the same size. A coarse wire foreign object damage (FOD) screen was located just forward of the bellmouth, and a flow straightener followed the bellmouth. An area constriction of 2.24 occurs between the flow straightener and the inlet face of Rotor 1B. Downstream of Rotor 1B and associated with the test cell were exit guide vanes (EGV) designed to remove the swirl from the test facility. The exit air was split into two passages, one for coarse venturi flow metering and the other for fine venturi flow metering. Power to drive the test rotor was provided by a high-pressure non-condensing steam turbine rated at 15,000 horsepower.

\subsection{Instrumentation}

Inlet total temperature was measured by 24 thermocouples distributed on the inlet screen. Inlet total pressure was measured by six pitot-static rakes, each with 7 elements (placed radially at centers of equal annulus area) located just downstream of the flow straightener. Traverse probes were used to measure blade element data at the rotor exit reference station (plane 1.51 in Figure 5.1). The probe was immersed to the five standard 
radial positions corresponding to 10-, 30-, 50-, 70-, and 90-percent of the annulus height at plane 1.51. Immersions at the inlet to the rotor reference plane (plane 0.95 in Figure 5.1) upstream of the rotor were established to correspond to the radial locations of design streamlines. These design streamlines passed through the previously specified immersions at the rotor exit reference plane 1.51. Static pressure taps were amply distributed on the casing and hub throughout the flow path. At a measuring traverse plane the static pressure taps were distributed circumferentially, but away from the traverse the static pressure taps were located at one circumferential location. Pressure and temperature probes were sufficiently insensitive to small pitch and yaw angles.

The distortion screen was located at plane 0.1 in Figure 5.1. The distortion screen covered a circumferential 90-degree segment of the annulus with a 0.016 inch wire diameter on a 20 mesh (Figure 5.2). The distortion screen was supported by a coarse screen with wires of 0.092 inch diameter, spaced 0.75 inches apart. This screen was designed to deliver a 90-degree one-per-revolution circumferential total pressure inlet distortion of 15-percent from the clean inlet at 100-percent corrected speed. Total pressure was measured at the inlet of Rotor $1 \mathrm{~B}$ with four distortion rakes placed at plane 0.86 in Figure 5.1. Blade element data was not taken during the inlet total pressure distortion test.

\subsection{Available Data from Rotor 1B}

Clean inlet data were obtained at 50-, 70-, 90-, and 100-percent corrected speeds. Blade element data were obtained by adjusting the data taken from the traverse probes, 
located upstream and downstream of Rotor 1B, (reference location plane 0.95 and plane 1.51, respectively, in Figure 5.1) to obtain conditions at the leading and trailing edge of the blade. The adjustment technique used measured total pressure, total temperature, static pressure, and the assumption that the shape of each meridional streamtube between a reference location and the blade edge remained fixed for all conditions. Of particular importance is the radial distribution of loss and exit relative blade angle this technique provided, which is necessary for constructing and calibrating the SLCC for TEACC source terms. Overall total pressure ratio and efficiency were provided at 50-, 70-, 90-, and 100-percent corrected speeds.

Distorted inlet data are known only at 50-, 70-, and 100-percent corrected speeds for a 90-degree one-per-revolution screen. Overall performance (total pressure and efficiency) is given at the three corrected speeds. However, radial and circumferential distributions of rotor performance are available only at a single point on the 100-percent corrected speed line. Rotor performance (total pressure and total temperature) was also recorded as a function of circumferential extent at the 100 -percent corrected speed point.

All experimental data used for comparisons with the model was obtained from Seyler and Gostelow, 1967. The uncertainty of this data was not discussed in the reference. The methods of obtaining the pressure and temperature measurements, however, are identified as pressure probes and thermocouples, respectively. Although it is impossible to definitively conclude the uncertainty of the data from this information, it is expected the uncertainty should be approximately the same as for other reported 
pressure and temperature measurements from similar instruments. A review of reference Thompson and Abernethy (1973) indicates that the uncertainty in the pressure measurements can range from \pm 0.0175 to \pm 0.5 psia, and the uncertainty in the temperature measurements can range from $\pm(0.38 \%$ reading $+2 \mathrm{R})$ to $\pm(0.75 \%$ reading $+4 \mathrm{R})$. 


\subsection{Consistency Check for Clean Inlet TEACC with Experimental Data}

The purpose of this chapter is to compare TEACC with experimental data for a clean inlet. As discussed in Chapter 5, the vehicle for comparison is NASA Rotor 1B with a three-dimensional grid of $(69 \times 13 \times 26)$. TEACC was tailored to simulate Rotor 1B by calibrating the streamline curvature code (SLCC) used to calculate turbomachinery source terms for Rotor 1B. This calibration was done by specifying a radial distribution of loss and exit relative flow angle from experimental data as a function of corrected mass flow and by specifying a radial distribution of blockage as a function of corrected mass flow as outlined in Appendix E. First, a three-dimensional grid is constructed and refined for grid convergence. Second, TEACC is compared with experimental data for a clean inlet.

\subsection{Grid Development}

TEACC requires a fixed three-dimensional grid on which to resolve the conservation equations (Equations 3.1, Section 3.1). The source terms were developed with a particular grid construction that modeled the bladed region of the compressor. After basic grid constraints were met, it became imperative to further refine the grid so that the flow solution was within a specified tolerance (grid convergence). Grid convergence was obtained by developing an initial three-dimensional grid sophisticated enough to model the flow field, and then modifying the grid separately in each of the three primary indices until the mass weighted total pressure ratio and the mass weighted 
efficiency varied within 2-percent difference with experimental data. Comparisons were made with the experimental results as well as the coarser grid solution to verify convergence in the right direction. Other criteria, which will be discussed, contributed to the decision on the final grid configuration. The grid selected from this investigation became the grid used for all clean and distorted flow-field analysis.

Grid construction in general could not be done just once for all applications. Therefore, the overarching grid construction constraint was for the grid to represent the physical boundaries of the machine being modeled (Rotor 1B). The outer and inner boundaries of the grid were flow-field boundaries defined by the NASA test facility. The upstream and downstream extent of the grid was defined by the given test geometry in Figure 5.1. An axial-radial view of the three-dimensional grid modeling Rotor $1 \mathrm{~B}$ and the test facility is given in Figure 6.1 with $(69 \times 13 \times 26)$ grid points in the axial, radial, and circumferential directions, respectively. This grid will be known as the base grid to which all grid refinements will be compared. A center-body consistent with the test cell was defined along the inner flow boundary for the entire extent of the grid.

Other constraints were related to the blade shape where the inlet and exit of the blade was defined as slanting lines in Figure 6.1. Most data for Rotor 1B were taken at the reference locations upstream and downstream of the rotor. An important restriction was that the computational domain had to be vertical at these reference locations for direct comparison with experimental data. Later, the selected grid was used for inlet distortion analysis. Therefore, the location of the screen simulation was accounted for 
with each grid. The grid was constructed with vertical grid lines in the vicinity of the screen to model the experimental distortion screen properly. Grid lines were smoothly packed through the bladed region where the flow was known to have strong gradients to reduce numerical losses. A radial-circumferential view of this base grid is presented in Figure 6.2 where a cylindrical right-handed coordinate system has been used with uniform circumferential segments. An integer number of grid segments has been constructed to conveniently model a 90-degree, one-per-revolution inlet distortion. Each of the four quadrants was designed to have an odd number of radial grid points so the flow field could easily be resolved in the center of each 90-degree quadrant. The grid in the circumferential direction was overlapped exactly by one segment to impose the circumferential periodic boundary condition. Each segment was therefore 15-degrees in circumferential extent with a total of 24 segments ( 6 segments in each quadrant) within a circle.

A grid density investigation was conducted by doubling the grid separately in each index and the results are presented in Appendix F. This study revealed that the radial distribution of the base flow field was within 2-percent difference of that produced by the increased grid density. However, the difference from experimental data in overall total pressure ratio and efficiency was roughly halved (from -1.06 percent to -0.41 percent difference in total pressure ratio and from -2.88 percent to -1.47 percent difference in total temperature ratio) by doubling the grid in the circumferential direction. This improvement was found to be directly related to halving the error in cross-sectional area 
and mass flow by doubling the circumferential grid. This means the Cartesian representation of a cylindrical cross section doubled in accuracy with the denser grid in the circumferential direction. The base grid was selected for all subsequent calculations since the improvements offered by the grid refinements were not significant enough to give up the faster wall clock time to discrete solution.

\subsection{Clean Inlet Comparison of TEACC with Experimental Data}

The available experimental data provided the opportunity to compare many overall flow-field quantities, as well as radial quantities. As part of the comparison of TEACC with experimental data, three variables, total pressure, static pressure, and absolute velocity vectors were investigated throughout the flow field. This investigation established that the overall compressor simulation behaved properly based upon expected compressor operation. TEACC results were then compared with experimental data for overall performance in terms of total pressure ratio and efficiency for four corrected speeds. A typical point on the 100-percent corrected speed line was investigated by TEACC and compared with experimental data.

\subsubsection{Overall Verification of TEACC (Clean Inlet)}

TEACC was operationally verified using Rotor $1 \mathrm{~B}$ at 100 -percent corrected speed near the design throttle condition as shown in Figure 6.3. During clean inlet operation, each circumferential segment operates with the same axisymmetric solution, allowing the presentation of total pressure, static pressure, and absolute velocity vectors to be conveniently compared on the same overall compressor geometry. A wire frame of Rotor 
1B's overall geometry is presented as a reference point for proper orientation of the presented variables, and to allow an immediate comparison of different variables at the same time. Total pressure is unchanged until the flow reaches the blade row. Internal to the blade, total pressure increases strongly, as expected. It is clear that total pressure is not uniform behind the blade, and this strong total pressure profile (increasing from hub to tip) remains throughout the exit of the simulation. Static pressure increases strongly through the bladed region, leaving a very nonuniform radial distribution in static pressure behind the blade. Such behavior is expected since, from the radial momentum equation, it can be observed that a change in circumferential velocity will cause a change in static pressure. Because of this radial variation in static pressure, an exit boundary condition that will properly support the strong and changing radial profile in static pressure is required, as was outlined in Chapter 3.

There are no stators at the exit of the rotor to turn the flow back toward the centerline; therefore, strong swirl is produced by the rotor and continues out the exit of the simulation. The swirl out the back caused no additional modeling constraints. In fact, since the swirl is relatively uniform at the exit, there are no strong circumferential gradients to cause undue numerical losses. Again, it is instructive to notice that the blades do not cause the flow field to swirl the flow upstream of the blades. This will become more important later in the discussion of flow field accuracy behind the distortion screen and in front of the blades. An overview of the rotor performance 
presented in the forgoing discussion demonstrates proper compressor behavior and encouraged pursuit of a more detailed investigation of TEACC's capabilities.

\subsubsection{Overall Performance Map (Clean Inlet)}

Calculated overall total pressure performance of Rotor 1B with a clean inlet is presented in Figure 6.4 and compared with experimental data. Four corrected speeds (50-, 70-, 90-, and 100-percent) were available for comparison, with symbols presented for the experimental data and lines for the TEACC solution. In an overall sense, there is good comparison between TEACC and the data. The maximum percent difference in total pressure ratio is less than one percent difference for each characteristic.

TEACC's overall efficiency calculations for Rotor 1B are compared with experimental data in Figure 6.5. The overall shape and character compares well with the experimental data. Each efficiency curve has a peak value which TEACC fails to reach, causing a maximum percent difference (for all speeds) between TEACC and experimental data of -2.9 percent occurring at the 50-percent corrected speed line.

\subsubsection{Radial Comparison of TEACC with Data (Clean Inlet)}

Since experimental data were available at the two reference locations before and after the blade as defined in Figure 5.1, TEACC results were compared to experimental data at these same two reference locations. The technique for running TEACC to a particular steady-state point was to vary the exit static pressure specified at the center of the flow field until the correct mass flow was reached. 
The variables presented in this section are compared to experimental data with the maximum percent difference of each variable presented in Table 6.1. A radial comparison was made with exit total pressure and total pressure ratio as presented in Figures 6.6 and 6.7 for the 100-percent speed point near the design throttle line. The overall shape is quite complicated for exit total pressure, ranging from about 22.5 psia at the hub to nearly $26.0 \mathrm{psia}$ at the tip, with inlet total pressure approximately uniform at 14.696 psia. Hub calculations shifted down from experimental data by -1.34 percent. The tip and overall shape are in good agreement with the experimental data. The total pressure ratio is compared with data in Figure 6.7 where a wide radial range in total pressure ratio can be seen. There is good agreement (1.0-percent difference) between TEACC results and experimental data, even though the compressor was not producing a uniform exit flow field. An investigation of exit static pressure and exit Mach number was necessary to determine the cause of the strong distribution in total pressure ratio. As shown in Figures 6.8 and 6.9, the exit Mach number and the exit static pressure are in good agreement with the experimental data, and the exit Mach number is fairly uniform, but the exit static pressure varied strongly, from 17 psia at the hub to around 20 psia at the tip. The largest difference for both exit Mach number and exit static pressure with experimental data is at the tip where Mach number is underpredicted by 2.7-percent and static pressure is overpredicted by 1.9-percent.

A radial comparison with experimental data can also be constructed for the exit total temperature (Figure 6.10) and total temperature ratio (Figure 6.11) for the 
100-percent speed point. Exit total temperature compares well (within 1.0-percent) with the data, with the greatest variation taking place near the tip. A similar variation at the tip appears in the total temperature ratio since the inlet total temperature is nearly constant at 518.67 R. Because the tip has the greatest total temperature ratio, it is clear that radial distribution of compressor work production is not uniform. The total temperature ratio has its largest deviation with experimental data of 1.1-percent near the tip (Figure 6.11).

Static temperature, which tracks the profile of the total temperature, is displayed in Figure 6.12. The agreement with data is good, with the only discrepancy occurring near the tip for a 1.9-percent difference. Static density is shown in Figure 6.13 as the only remaining equation-of-state variable to be investigated. The overall shape of the radial density profile is good, with the maximum variation from experimental data of 1.3-percent near the tip. The calculated experimental data density displayed a sharp turndown near the tip. The TEACC result also indicates a turndown near the tip, but not as sharp as the experimental data. This kind of discrepancy is expected since TEACC does not include casing hub and tip viscous effects.

Exit absolute velocity is compared to the calculated experimental data in Figure 6.14. For most of the radial extent, TEACC underpredicts the absolute exit velocity with the greatest deviation at the tip of -3.1 percent. A similar shape can be observed between exit absolute velocity and exit absolute Mach number (Figure 6.8). The exit circumferential velocity is presented in Figure 6.15. The middle of the flow field was predicted quite well with TEACC. However, the hub and the tip deviate from the 
calculated experimental data with the greatest exit circumferential velocity deviation of 7.7 percent at the hub. TEACC's ability to appropriately simulate turbomachinery performance for a clean inlet has been demonstrated by the good agreement with experimental data. 


\subsection{Distorted Inlet Comparison of TEACC with Experimental Data}

As stated in the introduction, total pressure distortion develops for a variety of reasons. The inlet testing community has developed sophisticated procedures for constructing screens which are placed in front of engines to represent the effects of a distorted inflow. Rotor 1B was tested with an inlet screen to quantify the effects of inlet flow distortion on its performance. Because the distortion data provided with Rotor 1B was based on a 90-degree, one-per-revolution screen, a simulation of the screen were used in the TEACC simulation. For this study, TEACC was compared at three different corrected speed lines for a distorted inlet. A single distortion point on the 100-percent speed line was investigated in great detail since radial and circumferential data were available. Overall performance of the distorted compressor is compared to experimental data, as well as radial and circumferential distributions.

\subsection{Distortion Screen Modeling}

To model the distortion screen, a special porous wall boundary condition (Pinker, 1967) was provided with NPARC. The boundary condition is semi-empirical in that experimental data had been taken across a variety of screens to establish a loss in total pressure as a function of Mach number, porosity, and Reynolds number (Figure 7.1a). The value of total pressure at the screen exit was calculated from the continuity, energy equations (total enthalpy equal to a constant), and the previously presented empirical pressure loss coefficient. The NPARC simulation is very flexible because a boundary 
condition can be imposed over any segment of the grid in or on the boundaries of the flow field. This feature makes it particularly convenient to impose the screen in the flow field corresponding to the physical location of the Rotor $1 \mathrm{~B}$ distortion screen investigation.

\subsubsection{Distortion Screen Simulation Overall Characteristics}

A single screen with a 90-degree, one-per-revolution pattern was designed to give a classical circumferential inlet total pressure inlet distortion to Rotor 1B. Porosity is defined as one minus blockage, and blockage is defined as the ratio of blocked area over total cross-sectional area of the annulus where the screen is located. Figure $7.1 \mathrm{~b}$ shows a schematic of the screen which was constructed from 0.016 inch diameter bars spaced 0.050 inches apart on a coarse structural mesh employing 0.092 inch diameter bars spaced 0.75 inches apart. This translates into a blockage of 0.5376 for the fine mesh and a blockage of 0.17554 for the coarse mesh. The porosity corresponding to these blockages was imposed as part of the semi-empirical boundary condition to model inlet distortion. At the 100-percent speed, the experimental data showed that the screen produced a total pressure loss of 15-percent from the clean inlet which was duplicated for the TEACC simulation.

The screen was expected to produce a reduction in the loss of total pressure ratio as the compressor speed was reduced. There was no experimental data to confirm the numerical magnitude of the loss in total pressure at other than the design speed, but the results obtained from the screen simulation appear to be reasonable. The screen's effects 
at three different compressor speeds are displayed in Table 7.1. The porosity calculated for the coarse mesh was imposed in the simulation, but the true porosity of the overlapping fine and coarse grids could not be obtained analytically. Therefore, a calibration of porosity was employed to give the known 15-percent loss in total pressure ratio at 100 -percent speed, which translated to a porosity of 0.51 . These porosity settings were maintained for all other distortion simulations. The coarse screen, designed to hold the fine screen structurally in place, contributed to a sizable 3-percent loss in total pressure ratio at the 100-percent speed, and falls to less than 1-percent at the 50-percent speed.

Investigations were conducted through the middle of the four circumferential quadrants located at the inlet and exit reference locations defined earlier in Figure 5.1. The screen was located in quadrant A (Figure 7.1b). The compressor rotates clockwise, causing air to swirl in the direction of increasing quadrant letter (A, B, C, and D).

\subsubsection{Distortion Screen Simulation Radial Characteristics}

Illustrated in Figure 7.2 are the radial characteristics of the distortion screen simulation and comparison to experimental results. The two larger graphs in Figure 7.2 each have four curves plotting the ratio of total pressure behind the screen divided by the average inlet total pressure versus radius for each circumferential quadrant. The experimental data are on the left and the TEACC simulation results are on the right. The character of the flow field behind the screen has certainly been captured, with Quadrant A (90-degree distortion) clearly apart from the other three quadrants. The individual 
pressure drops for Quadrants B-D calculated by the screen simulator are clustered together and represent the mean of the data in these quadrants. The four graphs at the bottom of Figure 7.2 show a detailed comparison of the screen simulator to experimental data for each of the four circumferential quadrants. Quadrants B-D have their maximum percent difference in pressure ratio around 4-percent, but Quadrant A has a maximum percent difference around 6-percent.

\subsection{Overall Rotor 1B Performance Comparison}

An overall total pressure ratio performance map of Rotor 1B is presented in Figure 7.3 for three corrected speeds of 50-, 70-, and 100-percent. Since there were no experimental data available for comparison at the 90 -percent speed, no simulation results are presented for this speed. The clean speed lines are presented to give a proper orientation of the distorted data with stall depicted for each speed line at its lowest corrected mass flow. A simple stalling criterion was imposed on the TEACC simulation. When any one of the circumferential segments acquired a corrected mass flow which exceeded stall for the clean inlet, the compressor was considered stalled. Likewise, the TEACC simulation was halted when any one of the circumferential segments exceeded the clean inlet data on the choked end of the speed lines. These two restrictions define the distortion calculation limits presented for each speed in Figure 7.3.

For the two low-speed cases, the clean and distorted data lay close together. The extent in corrected mass flow is about the same for the simulation and the experimental distortion data. An enlarged view of the map with distortion at the lower speeds is 
displayed at the bottom of Figure 7.3, showing that the simulation does a better job of calculating performance with distortion near the middle of the extent than it does when part of the compressor is operating very near either the stalled or the choked end of the compressor characteristic. The maximum percent difference between TEACC results and experimental data at the lower speeds was approximately 2-percent.

The results for 100-percent speed are more interesting because the experimental data presented a region where the compressor intermittently stalled or remained stable with the imposed inlet distortion screen. The TEACC simulation missed the corrected mass flow by a maximum of 3-percent. It is interesting that the TEACC simulation maps out a range which includes approximately the middle of the intermittent stall region.

Overall efficiency is compared to experimental data in Figure 7.4. In all cases TEACC overpredicts the efficiency compared to the experimental data. The three graphs at the bottom of Figure 7.4 (one for each corrected speed) give detailed comparisons of TEACC results with data. The maximum percent difference between TEACC results and the experimental data is located near the stall or choked portion of each corrected speed. The maximum percent differences between TEACC results and data are 6.9-, 4.5-, and 5.9-percent difference for 50-, 70-, and 100-percent speeds respectively.

There are two reasons for the TEACC simulation to miss the experimental data, assuming the data are correct. Since the screen failed to match the experimental data by a maximum of 5.74-percent in the distorted region, it is reasonable to attribute part of the discrepancy between TEACC and the experimental data to the inaccuracy of the screen 
simulation. However, the primary reason for TEACC results to deviate from the experimental data can be associated with correlations that are radially inflexible to the changing flow field. As stated in the beginning of this chapter and outlined in Appendix E, the SLCC is calibrated to a clean inlet radial distribution of loss and exit relative flow angle as a function of corrected mass flow. As the flow field changes due to inlet distortion, the radial distribution of loss and exit relative flow angle would be expected to vary with the local flow. A set of loss and exit relative flow angle correlations sensitive to the local flow field (especially inlet and exit velocity profiles) would automatically adjust the loss and exit relative flow angle. From the introduction (Chapter 1.0), Greitzer and Strand (1978) demonstrated that total pressure inlet distortion is threedimensional; therefore, the radial distribution in loss and exit relative flow angle in the presence of inlet distortion would be expected to be different from that produced by a clean inlet at the same mass flow. The current TEACC simulation provides the lower boundary of accuracy to what would be expected from TEACC with a proper set of correlations.

\subsection{Radial Comparison of Compressor with Experimental Data}

This investigation now turns to an examination of TEACC results behind the compressor at the second reference plane. In Figure 7.5, exit total pressure (defined by the ratio of exit total pressure over the average screen inlet total pressure) versus compressor radius is plotted with experimental data on the left and TEACC results on the right. This pressure ratio is characterized by a division between Quadrant A and the 
other three quadrants. TEACC does a good job of matching the character of the exit total pressure in Quadrant A with experimental data. The TEACC results agree with the experimental data in the general character of the other three quadrants and identifies Quadrant B as containing the largest exit total pressure. The experimental data also confirms the TEACC results which show exit total pressure decreasing steadily from Quadrant B to Quadrant D. Although the shape of the other three quadrants is moving in the correct direction, the extent and scatter with pressure ratio is missing. Shown at the bottom of Figure 7.5 is a detailed comparison of each circumferential quadrant of TEACC with experimental data. The TEACC results in Quadrant A compare well to the experimental data, except for the hub point, which results in a maximum percent difference in pressure ratio equal to 6.15-percent. TEACC results compared to experimental data are too low for Quadrant B (6.82-percent) and too high for Quadrants C (5.86-percent) and D (8.64-percent). The highest maximum percent difference is within 8.7-percent in quadrant $\mathrm{D}$.

The final comparison with experimental data is also behind the compressor (same reference location as exit total pressure). In this comparison of TEACC results with experimental data, Figure 7.6 is arranged like the previous figure (Figure 7.5). The ordinate for each graph is the ratio of total temperature at the compressor exit divided by the average total temperature at the screen inlet. Quadrant A is again characterized as having a greater slope than the other three quadrants, and TEACC identifies the overall flow character well. TEACC correctly shows, in the nondistorted region, that the exit 
total temperature was the highest in Quadrant B with the other quadrants decreasing in value from Quadrant B-D. TEACC's calculations for the total temperature at the compressor exit have a greater spread in Quadrants B-D than the exit total pressure (Figure 7.5). Comparisons of the individual circumferential graphs at the bottom of Figure 7.6 show that the hub of Quadrant $\mathrm{A}$ is not as steep a slope as the experimental data, but the tip matches the data very well. Quadrants C and D match the data well within 1.81-percent and 2.19-percent, respectively, and even Quadrant B matches the data well, except at a single tip point. The largest percent difference between TEACC results and experimental data for exit total temperature is 6.60-percent. 


\subsection{Observations and Lessons Learned}

During the comparison of TEACC with experimental data, insight was obtained into the physical phenomena surrounding compressors and the characterization of distortion flow fields. The areas which will be discussed are: the influence of source terms on the flow field, the screen simulation, and the effects of swirl on the flow field. Source terms represent a new way to investigate the performance of a compressor and will be presented for a clean and distorted inlet. This is also an opportunity to investigate the flow field around the screen simulation and determine the character of the flow field produced. Finally, the flow field through the compressor with inlet distortion was investigated to determine the effect of swirl and the radial characterization with distortion.

\subsection{TEACC Source Terms}

This section expands on the significance of implementing the turbomachinery source terms within TEACC. These source terms provide insight, such as relative differences between axial, radial, and circumferential force and rate of work distributions, not obtainable from the usual investigation of flow, pressure, and temperature. . Such insights are possible because the source terms represent the direct effect of the compressor on the flow field. A source term analysis is presented for both a clean inlet and a distorted inlet to provide the radial and circumferential character of the compressor. 


\subsubsection{Clean Inlet TEACC Source Terms}

The clean inlet source term investigation was conducted at the same 100-percent overall flow point as that of the radial comparison of TEACC with experimental data. First, the sources will be presented as they were calculated for the TEACC simulation on a per local volume basis as a function of radius. Second, the sources are multiplied by local volume to investigate the radial distribution of forces and rate of shaft work production over a single 15-degree circumferential segment. Finally, the sources are integrated over the flow field to determine the cumulative forces and cumulative rate of shaft work produced by the compressor at this high rotor speed operating point.

The compressor was simulated with the use of source terms in the form of forces in the axial, radial, and circumferential direction and rate of shaft work, all on a per volume basis. These source terms are shown in Figures $8.1-8.4$ as a function of nondimensional radius. Some general comments about all four source terms are in order. The forces per volume primarily increase from hub to tip, with the axial force per volume (Figure 8.1) showing the greatest increase. The circumferential force per volume (Figure 8.2) and the radial force per volume (Figure 8.3) start to turn down near the tip. The radial force per volume shown in Figure 8.3 is upwards of one order of magnitude smaller than the axial and circumferential forces. The rate of shaft work per volume in Figure 8.4 is strongly increasing with a slight turndown near the tip.

If the volume distribution as a function of radius is known the axial, radial, and circumferential forces and rate of shaft work can be calculated. The forces and rate of 
shaft work per volume (Figures $8.1-8.4$ ) are multiplied by local volume to produce blade forces and rate of shaft work on a 15-degree segment, as shown in Figures 8.5 - 8.8. The technique to calculate forces equal to pressure-area forces and inertial forces was shown in Section 3.2. The axial forces and their parts, the pressure-area forces and the inertial forces, are presented in Figure 8.5. It is instructive to observe that the axial forces are largely comprised of the pressure-area forces. Since the axial velocity (Figure 8.9) decreases through the compressor, the inertial force is negative, causing the axial force to be less than the pressure-area force. The axial force is approximately halved at the inner and outer casing due to a smaller volume produced by a finer grid near the radial boundaries. The largest force is located near the tip.

The circumferential force, like the axial force, increased primarily from the hub to the tip of the compressor (Figure 8.6). As was described in Section 3.2, the circumferential forces are constructed only from inertial forces. The largest circumferential force is located near the tip; however, the cumulative circumferential force for the machine is zero, since the circumferential forces produced by the compressor 180-degrees apart are equal in magnitude and opposite in direction.

The radial force is constructed with pressure-area forces and inertial forces, as outlined in Section 3.2. The pressure-area forces remain positive but decrease toward the tip. The inertial forces are a strong function of the radial velocity through the compressor, as presented in Figure 8.10. The radial velocity at the hub of the compressor is largest, because of strong ramping of the hub geometry through the compressor. The 
radial velocity is negative at the outer casing, due to the outer casing geometry decreasing through the compressor. Since the exit velocity minus the inlet radial velocity is negative for the hub region and positive for the tip region, the radial inertial force tracks this velocity difference, as can be seen in Figure 8.7. This means the radial pressure-area force and inertial force together produce the largest radial force near the tip. The radial force is clearly more than an order of magnitude less than the axial and circumferential forces. The cumulative radial force for the machine is zero since the radial forces on the compressor, 180-degrees apart, cancel.

The rate of shaft work strongly increases from hub to tip, as shown in Figure 8.8. This strong increase in rate of shaft work with radius was expected with the observation that total pressure ratio (Figure 6.7) and total temperature ratio (Figure 6.11) also increased with radius through the compressor. The rate of shaft work versus radius has its largest value near the tip.

\subsubsection{Distorted Inlet TEACC Source Terms}

The following analysis of turbomachinery source terms was conducted at the same $100 \%$ corrected speed point used to compare TEACC with experimental data for inlet distortion. Presented in Figures $8.11-8.14$ are the turbomachinery source terms as a function of nondimensionalized compressor radius at the centers of the control volumes through the bladed region. The axial forces per volume are presented in Figure 8.11 where the axial forces per volume are the greatest in the distorted region. The axial forces are the smallest in the quadrant adjacent to the distorted flow field and prior to 
entering the distorted region (Quadrant $\mathrm{D}$ ). In Figure 8.12, radial forces per volume can be observed to have the same trend as axial forces per volume. Both axial forces per volume and radial forces per volume after the distorted region have values which decrease in the direction of swirl until the distorted region is reached. Circumferential forces per volume and rate of shaft work per volume are presented, respectively, in Figures 8.13 and 8.14. Both the circumferential forces per volume and the rate of shaft work per volume display the same character where their values for Quadrant A cut across the other quadrants' values. But an overarching observation can be made for each of the source terms in Figures 8.11-8.14, that the compressor decreases in performance in the direction of rotation for Quadrants B-D, and Quadrant A, with the inlet distortion, produces the greatest amount of force and work.

\subsection{Investigation of the Flow Field around the Screen}

The following analysis of the flow field around the screen was conducted at the same 100-percent corrected speed point as that used in the distortion comparison of TEACC with experimental data. The screen's effect can best be seen in the screen exit total pressure presented in Figure 8.15. This figure clearly shows a drop in total pressure between the distorted quadrant (Quadrant A) and those described by the other three quadrants. Referenced to the clean inlet, total pressure dropped 15-percent across the fine screen as was designed over the 90-degree circumferential quadrant, and 3-percent over the coarse screen for the remainder of the cross section. Total temperature was left unchanged, as expected, after crossing the screen. 
The upstream static pressure in Quadrant A for the distorted region in Figure 8.16 has increased due to the low subsonic flow approaching the downstream screen blockage. The inlet static pressure on the adjacent two quadrants (Quadrants B and D) are nearly the same, with inlet static pressure in Quadrant C close to Quadrant B and D. The difference in static pressure in the lightly distorted regions can be associated with a difference in circumferential velocity. Figure 8.17 shows that in Quadrant $\mathrm{A}$, the magnitude of the absolute inlet velocity decreased from the other quadrants, which is consistent with the previously observed increase in inlet static pressure. The exit static pressure in Figure 8.18 is very similar in shape to the exit total pressure previously presented in Figure 8.15. The screen caused a loss in exit static pressure in Quadrant A, while the exit static pressure in the other quadrants remains nearly identical.

Inlet and exit Mach number have a consistent shape with inlet and exit velocity, and they are presented in Figures 8.19 and 8.20 to show the Mach numbers of the flow moving through the screen. As expected, the decrease in exit static pressure (Quadrant A) is consistent with a relative increase in exit Mach number from the screen inlet. Each of the figures presented so far has Quadrants B and D behaving symmetrically and nearly identical while Quadrant C is noticeably different. These differences can be explained in part by Figure 8.21, which is a plot of exit circumferential velocity. Quadrants A and C are plotted very near zero, meaning there is no circumferential velocity. However, the quadrants on either side of the strong distortion (Quadrants B and D) have a nearly symmetric circumferential distribution in circumferential velocity. This is expected 
because the strongly distorted region has a much lower exit static pressure than the other quadrants, and the circumferential flow on either side is moving toward the low static pressure quadrant (Quadrant A). Figure 8.22 shows the plane of symmetry passing diagonally through the middle of the distortion screen (Quadrant A) and the middle of Quadrant C. The flow is modeled as traveling toward the low static pressure region behind the distortion screen. The circumferential flow field is not completely symmetric. This can be explained by observing that the compressor is only a quarter rotor diameter downstream of the screen and the compressor passes a very small swirl component back upstream.

\subsection{Compressor Performance}

This section focuses on the flow field in the distorted region of the compressor blades. These TEACC results are from the same $100 \%$ speed distorted point previously presented with screen performance. Although some degree of symmetry is expected to persist ahead of the blades, all symmetry is lost due to the strong swirl produced by the compressor blades in the presence of inlet distortion. The strong swirling flow field produced by TEACC was investigated for the overall character of the flow field in two ways: first, in the distorted region, and second, outside the distorted region.

\subsubsection{Investigation of the Effect of Swirl on the Performance of the Compressor with Inlet Distortion}

The circumferential velocity within the flow field caused by the high rotor speed compressor blade is a direct result of the compressor blade performing work on the flow. 
The blade causes a strong circumferential velocity which establishes the location of the largest static and total pressure regions. The streamlines through the distorted and undistorted regions of the compressor will be investigated, as will the effect of swirl on the transport of total pressure and total temperature through the compressor.

The effect of the screen is displayed in Figure 8.23. The compressor exit total pressure is presented in Figure 8.24. Notice that the highest value in total pressure is not in the center of the distorted region but has shifted clockwise and has its largest value near the tip. The exit total temperature, displayed in Figure 8.25, has shifted clockwise but is not as pronounced as the exit total pressure. Figure 8.26 presents the static pressure which resembles that of the previously presented total pressure. The highest static pressure is close to the interface of Quadrants A and B. It is important to recall that the clockwise circumferential velocity presented in Figure 8.27 shows the strong rotation of the fluid due to the compressor rotating clockwise relative to the primary direction of flow. Where the performance is high behind the distorted region, the velocity vectors at the tip can be recognized to be larger than in the undistorted regions. These velocity vectors can be converted to streamlines which show the strong effect of swirl downstream of the compressor.

The streamlines are shown in Figure 8.28. A schematic of the distortion screen is presented to indicate inlet distortion (Figure 8.28a); however, this time, the labeling is slightly different than before, with Segment A defined in the middle of Quadrant A and Segment $\boxplus$ chosen to represent a segment just before the rotor begins to sweep through 
the distortion in Quadrant D. An important distinction is that Segment A is in the distorted region and Segment $B$ is in the undistorted region. The upper right-hand picture in Figure 8.28b shows in simple terms how Quadrant A and Segment $Ð$ are behaving on the compressor map. First, Segment $\boxplus$ operates in the clean inlet region of the compressor, producing a pressure ratio with a high corrected mass flow, but Quadrant A operates through the distorted screen where the total pressure in front of the compressor is low, causing a reduction of corrected mass flow. Quadrant A operates higher on the compressor map closer to stall, which means that approximately 75 -percent of the compressor operates near choke while 25 -percent of the compressor operates near stall. The portion of the compressor operating behind the distortion produces more work than any other part of the compressor. It is clear from the rate of shaft work per volume presented earlier (Figure 8.14) that the total pressure ratio across the compressor is the highest in the distorted region.

The streamline in the bottom view of Figure $8.28 \mathrm{c}$ tells more about the compressor operation. Segment $\boxplus$ describes the streamlines to the right of the distorted region while the blade turns the flow strongly toward and behind the distorted region. Quadrant A, on the other hand, moves through the middle of the distorted region. Again, the compressor strongly swirls the flow, but the total pressure increase through the distorted region is greater than that of Segment $\square$. The flow field at the compressor exit is convected clockwise by the swirling flow to an adjacent part of the compressor, and the streamlines in Segment $\boxplus$ show that the lower total pressure fluid is convected behind 
the compressor in the distorted region. This is a further indication of the clockwise transport of total pressure attributable to the swirling flow, and an indication that the total pressure behind the compressor is not uniform, but increasing in the direction of flow rotation. The performance increase, which begins at the distorted region, increases the static pressure behind the compressor and convects total temperature and total pressure with the flow to an adjacent point behind the compressor. This action causes the adjacent point to further backpressure the compressor in this region, subsequently increasing its performance and moving that region closer to stall. This increase implies that the swirl will cause the strongest backpressuring of the distorted region to be transported clockwise behind the adjacent non-distorted region (intersection of Quadrant A and B), increasing performance in this region, and producing the largest total pressure found anywhere in the compressor, as shown in Figure 8.24. Therefore, the total pressure increases clockwise through the distorted region.

The transport of total pressure and total temperature clockwise through the compressor can be viewed in a slightly different way in Figure 8.29. Here, a radial massweighted average of total pressure and total temperature is presented versus the circumferential extent at the upstream and downstream reference locations. A schematic of the distortion screen is at the top of the page and centered over Quadrant A in each plot. Inlet total pressure (Figure $8.29 \mathrm{~b}$ ) to the compressor can be observed to have two different uniform regions, one behind the distortion screen (Quadrant A) and the other composed of the remaining three quadrants, which have higher values of inlet total 
pressure (Quadrants B, C, and D). The compressor exit total pressure increases steadily through the distorted region, with its highest value at the beginning of Quadrant B. The ratio of total pressure (Figure 8.29a) also increases steadily in a clockwise direction through the distorted region, due to the compressor transportation of total pressure clockwise through the distorted region. This increases the static pressure behind an adjacent portion of the compressor in the clockwise direction, backpressuring this portion of the compressor and producing a performance increase. This increased performance shows itself as an increase in total pressure ratio.

A similar story can be constructed with total temperature at the compressor inlet and exit. The inlet total temperature to the compressor remains uniform in the presence of the screen, as shown in Figure 8.29d. The exit total temperature, like the exit total pressure, steadily increases clockwise through the distorted region. This means the total temperature ratio in Figure $8.29 \mathrm{c}$ also increases through the distorted region. Total temperature ratio, like total pressure ratio, reaches its largest value at the interface of Quadrant A and Quadrant B because of the swirl effect through the distorted region.

\subsubsection{Investigation of the Radial Characterization of the Flow Field with Inlet Distortion}

The earlier investigation of the screen revealed a total pressure drop of $15 \%$ at the inlet flow field to the compressor, with the total temperature remaining unaffected across the screen. The compressor exit flow field is strongly affected by the compressor and the inlet distortion in Quadrant A. The radial variation of exit total temperature is presented in Figure 8.30 where the total temperature in Quadrant A cuts across the total temperature 
of the other 3 quadrants. However, the value of total temperature in each of the quadrants after Quadrant A decreases for all radial locations from hub to tip. This trend is also shown in total temperature ratio in Figure 8.31. The shapes of the exit total temperature and the total temperature ratio look the same since the inlet total temperature is uniform. Figure 8.32 shows a reduction in exit total pressure behind Quadrant A, due to the lower inlet total pressure. The total pressure in the other three quadrants also generally decreases in the direction of rotor rotation. Figure 8.33 presents total pressure ratio in Quadrant A as significantly higher than the rest of the compressor because the distorted region operates on the overall compressor characteristic, closer to stall. This was discussed earlier during the investigation of swirl through the distorted region of the compressor. Outside the distorted region of the compressor, total temperature and total pressure decrease in the direction of compressor rotation; hence, the direction of swirl. Velocity and static quantities must be examined to determine why the totals drop clockwise through the compressor, away from the distorted region.

The inlet swirl is shown in Figure 8.34 where the effects of the rotating compressor are discernible in the hub region in Quadrants B and D (those nearest the distorted region). Most of the change in swirl is associated with the flow moving toward the low-pressure region behind the screen. The inlet swirl is still fairly symmetric, with a slight shift in swirl in the direction of rotor rotation. The swirl leaving the compressor, shown in Figure 8.35, has increased from nearly zero to $450 \mathrm{ft} / \mathrm{sec}$ on the average. The strong swirl at the compressor exit is not very different for Quadrants B-D. The exit swirl 
in the distorted region is, on the average, just as strong as the other quadrants, but the shape is reversed, causing the hub to have the lowest swirl and the tip to have the highest swirl. This behavior is consistent with the total temperature ratio (Figure 8.31), where the hub produces the least work and the tip produces the most work. With such a sudden increase in swirl velocity, the absolute exit velocity must also increase.

Inlet and exit absolute velocity are presented in Figures 8.36 and 8.37, showing that a dramatic increase in velocity occurred from approximately $560 \mathrm{ft} / \mathrm{sec}$ to, on the average, $750 \mathrm{ft} / \mathrm{sec}$. Low inlet absolute velocity due to the screen in front of the distorted region is to be expected. The exit absolute velocity is so influenced by the exit circumferential velocity that their shapes are very similar in Quadrant B-D. Since total pressure is low behind the distorted part of the compressor, absolute exit velocity also remains low. However, the exit absolute velocity at the tip approaches the tip velocities of the non-distorted quadrants, consistent with the exit swirl velocity (Figure 8.35 ) being highest in this region. Exit Mach number in Figure 8.38 is also very similar in shape to the exit absolute velocity (Figure 8.37). Outside the distorted region at the compressor exit, the circumferential velocity is very strong, and dominates the overarching shape of the velocity profiles. These velocity profiles are very similar in Quadrants B-D, suggesting that the decreases in total pressure and total temperature observed previously are primarily due to a decrease in static quantities.

Inlet static pressure to the compressor is shown in Figure 8.39. Here, the static pressure in front of the distorted region is noticeably lower, due to the low inlet total 
pressure produced by the screen. The static pressure in Quadrants B-D is very similar to the static pressure in Quadrants B and D and lower than Quadrant C due to the symmetric swirl flow around the screen. The exit static pressure presented in Figure 8.40, along with the totals, gives the most insight to compressor behavior. Static pressure follows the same trend observed from the totals presented earlier where static pressure steadily decreases in the direction of rotor rotation. This trend is observed in exit static temperature as shown in Figure 8.41. Here, the exit static temperature in Quadrants B-D steadily decreases and maintains a shape consistent with exit total temperature. The overarching character of the flow field can be described by combining the compressorproduced swirl with compressor performance as described below.

As presented earlier, the compressor performs the most work in the distorted region where it operates near stall. Due to swirl, the compressor convects high-energy fluid at the compressor tip clockwise out of the distorted region into the first part of Quadrant B. With the addition of this high-energy fluid, the already undistorted totals in this region are made even higher, producing the previously observed highest total pressure. Along with the high exit total pressure, the static pressure is also consistently high. This high static pressure backpressures the compressor in this region, causing it to do more work. However, in the middle of Quadrant B, the high-energy swirl produced from the distorted region no longer directly contributes to the compressor performance since there is no inlet distortion in this quadrant. Therefore, the exit total pressure decreases, also causing the exit static pressure to decrease. With less backpressuring of 
the compressor, the compressor does less work and the exit total pressure decreases. Lower total pressure is convected clockwise to adjacent quadrants. Therefore, because of the lower static pressure, the total pressure produced by the compressor steadily decreases in the direction of rotation, causing the exit static pressure of an adjacent segment due to swirl to also decrease steadily. This phenomenon continues until the distorted region is reached. 


\subsection{Summary and Conclusions}

The primary methodology and significant results of this study are:

- A three-dimensional time-dependent turbomachinery methodology and code was developed for the investigation of complex inlet distortion.

- The code, TEACC, is constructed by modifying the Euler equations to include turbomachinery source terms which are calculated from a SLCC.

- TEACC's comparison with experimental data producing good results for both a clean and distorted inlet using a single rotor with total pressure inlet distortion.

- A coarse grid can be used with the TEACC methodology for efficiently calculating turbomachinery flow fields.

- A variable relaxation factor applied to the sources transformed the turbomachinery simulation to a stable and robust solution procedure.

- Inlet total pressure distortion screens are simulated by a general distortion capability.

- In the direction of rotor rotation, swirl causes the compressor loading to increase in the distorted region and decrease in the non-distorted region.

The investigation of previous work clearly shows the increasing demand for improved simulation development to enhance the analysis and design of complicated turbomachinery. These tools often provide the ability to discover the flow field produced by turbomachinery without the expensive bending of metal and test time in an air- 
breathing test facility or in flight. Inlet distortion produces a complicated 3D flow field which requires the modeling of the entire compressor. TEACC has been developed to model inlet distortion through the use of semi-actuator disk theory. This technique allows efficient computational grids that provide a practical solution for inlet distortion problems. TEACC was constructed by developing a methodology to merge two proven technologies, NPARC and a SLCC, into a single turbomachinery simulation. NPARC was modified from a 3D Navier-Stokes duct flow simulation to an Euler solver able to support turbomachinery source terms. An implicit integrator was provided with NPARC, along with boundary conditions. A uniform exit static pressure boundary condition was found to be inadequate with a strong distribution of exit swirl. However, a variable exit static pressure boundary condition based on satisfying the conservation equation one axial station upstream of the exit provided the necessary flexibility in the presence of swirl. The streamline curvature code, the other proven technology, was modified by truncating the grid on either side of the blades, providing a highly responsive 2D compressor simulation based on dynamic boundary conditions. These boundary conditions were obtained from NPARC on either side of the compressor blade so that turbomachinery source terms could then be calculated from the SLCC and implemented into the NPARC.

The TEACC construction methodology further established a procedure for distributing turbomachinery source terms, which were distributed in the radial direction from direct application of the SLCC. The circumferential distribution of source terms 
was also established from an application of the SLCC through the use of locally responsive boundary conditions calculated on either side of the blade from the changing circumferential flow field. The axial distribution of source terms was applied through the use of a distribution function. A uniform distribution function provided the most robust turbomachinery simulation by keeping the relative change in sources between adjacent bladed control volumes and the maximum value in sources also at a minimum. Of particular importance was the development of a variable relaxation factor which allowed the simulation to be robust and remain stable near choked flow, where the radial distribution of loss and exit relative flow angle characteristics become vertical. This variable relaxation factor was established as a geometric progression through all nine axial control volumes, with the range of the initial relaxation factor between 0.5 and 1.0 .

The TEACC simulation was compared with the experimental data from NASA Rotor 1B because of the simplicity of a single rotor and the radial distributions of data available for clean and distorted inlet operation. No correlations were available to model loss and exit relative flow angle as a function of local flow field; therefore, the radial distribution of loss and exit relative flow angle from clean inlet experimental data at each corrected mass flow became the radial characteristics used by the SLCC to characterize Rotor 1B. The SLCC was calibrated by the use of blockage to match the radial distribution of experimental data in total pressure ratio and efficiency. The SLCC produced absolute and relative variables which compared well to experimental data overall (Figure E.12) and radially (Table E.1). 
A grid investigation was conducted to determine if the base grid of $(69 \times 13 \times 26)$ was fine enough to produce a solution sufficiently independent of the grid. This investigation revealed that doubling the grid in the axial or radial direction had little effect on the solution. However, doubling the grid in the circumferential direction roughly halved the error in overall total pressure ratio and total temperature ratio, and the radial distribution of the flow field remained basically unchanged. The improvement in overall total pressure ratio and total temperature ratio was attributed to the doubling in accuracy of the cross-sectional area calculations which were needed to calculate mass flow. Since the refined grids failed to produce a significant improvement in the radial distribution of the flow field, the base grid was used for all subsequent TEACC simulations of Rotor 1B.

TEACC was first compared with experimental data for a clean inlet and found to produce an "axisymmetric flow with swirl" solution. This allowed the grid simplification of using only five circumferential segments spanning part of the circumference to reduce the computational time to a converged solution by one quarter (approximately 15 minutes on a 200-MHz P6 PC). TEACC's overall results with experimental data compared within 1-percent for total pressure ratio and within 3-percent difference for efficiency. A radial comparison of TEACC results with experimental data also produced good comparisons (Table 6.1).

TEACC was also compared with experimental data for total pressure inlet distortion using Rotor 1B. The distortion was produced with a 90-degree, one-per- 
revolution screen simulated by a boundary condition provided by NPARC. TEACC was used to simulate Rotor 1B performance over three speed lines and produced a total pressure ratio which compared within 3-percent of experimental data and an efficiency which compared within 7-percent difference of experimental data. A detailed comparison of TEACC results with experimental data was conducted at a single point on the 100percent speed line because this was the only place radial and circumferential measurements were taken. The screen simulation produced a loss in total pressure which was within 5.74-percent of experimental data.

A radial comparison of TEACC results to experimental data revealed that TEACC models the overall character of the compressor well, and does a particularly good job of predicting the magnitude and shape of exit total temperature and exit total pressure in the distorted region (Quadrant A). TEACC calculated the overall character of exit total pressure and exit total temperature in the nondistorted quadrants, with the large value in Quadrant B decreasing clockwise through Quadrant D. In general, the TEACC-produced exit total pressure did not completely reach the energy highs in Quadrant B, and compared to experimental data they did not sink to the energy lows in Quadrant D.

The investigation of the flow field around and through the screen with the TEACC results confirmed that the screen simulation modeled the flow correctly. The flow field was nearly symmetric about a plane bisecting Quadrants A and C, with a decrease in total and static pressure across the screen in Quadrant A. 
A detailed investigation of the compressor flow field was conducted to evaluate the effects of the distortion screen simulation and to compare these results with experimental data. The study revealed that strong swirl convects total temperature and total pressure with the flow in the direction of rotor rotation. This increase in total pressure produced an increase in static pressure in an adjacent part of the compressor which backpressured the compressor, producing an increase in performance. Therefore, the greatest total pressure and total temperature is produced at the intersection of Quadrants A and B (transition from distorted to undistorted). The study also revealed that outside the distorted region, the strong swirl caused by the compressor produced the opposite effect of the distorted region, causing total temperature and total pressure to decrease from Quadrant B-D. After the distorted region, the compressor's performance decreased, causing a reduced total pressure and temperature to be convected with the flow to an adjacent segment of the flow. With the lower total pressure came a reduced static pressure which decreased the backpressure of the compressor, causing a further reduction in performance. This trend continued, producing the lowest total pressure and total temperature in Quadrant D (transition from undistorted to distorted). 


\subsection{Recommendations}

During the development of TEACC, several additional capabilities or limitations were noted but were outside the scope of this work. The following ideas are prioritized in importance and offered for further consideration:

(1) develop blade row loss and deviation (related to exit relative flow angle by the blade metal angle) correlations,

(2) extend to multiple blade rows,

(3) develop implicit boundary conditions and implicit source terms,

(4) include post-stall behavior, and

(5) apply to complex and dynamic distortion.

Recommendation (1): The most restrictive part of the TEACC simulation is the need to specify a radial compressor map specifying a distribution of loss and deviation. Work has been done in this area to produce correlations of loss and deviation. A study should be conducted to determine the most appropriate on- and off-design correlations for a given compression system. Flexible correlations would improve the TEACC circumferential inlet distortion simulation in two ways. First, detailed radial distributions of loss and deviation maps from experimental data would not be needed. This is particularly important because general loss and deviation information is seldom made available for general use. Second, the off-design flow field would acquire a more accurate distribution of loss and deviation than that specified by the experimental data because the correlations would be sensitive to the changing flow field. TEACC's radial 
and complex inlet distortion simulations would benefit the most from a general set of correlations because the loss and deviation profiles would be expected to shift the most from those of the clean inlet. It is unlikely that a single set of correlations will be flexible enough to model all compressors. This is particularly true with the blade design philosophy moving away from standard blade profile families. New blade designs are incorporating modern computational procedures that arbitrarily specify camber and blade thickness to produce a prescribed velocity distribution (Cumpsty, 1989). Therefore, a collection of correlations tailored to simulate a variety of compressors should be collected for ready use.

Recommendation (2): TEACC has successfully demonstrated its ability to model circumferential total pressure inlet distortion on a single rotor. This technology should be expanded to multiple blade rows to investigate inlet distortion problems on modern highspeed compressors. The first technology hurdle to accomplish this task has been completed by restricting the SLCC to operate only over a narrow region on either side of the compressor to calculate turbomachinery source terms. This idea would be expanded to cross each blade row separately for each circumferential segment, always looking to the local TEACC flow field for boundary conditions.

Recommendation (3): TEACC's boundary conditions were supplied with NPARC and they were a robust mixture of explicit zeroth and first-order boundary conditions. A potential benefit may be realized by extending the boundary conditions and the turbomachinary source terms from explicit to implicit, consistent with the 
implicit NPARC. Additionally, each boundary condition can be extended to first order in keeping with the theme of consistency. This kind of consistency was found to produce robust 1D solutions in ATEC (Garrard, 1995), allowing implicit run times for compressor simulations to be reduced by a factor of 50 .

Recommendation (4): TEACC can be further expanded into post-stall region by incorporating a stall simulation methodology developed in DYNTECC (Hale, 1992). DYNTECC calculates post-stall turbomachinery source terms from a control volume analysis and a first-order lag on the overall 1D characteristics (total pressure ratio and total temperature ratio versus corrected mass flow). This approach for modeling turbomachinery was extended to 3D by the development of a radial distribution of source terms (Chalk, 1996). The approach is to use the SLCC in the pre-stall region and the extended 3D DYNTECC methodology in the post-stall region. This would produce a 3D simulation capable of simulating surge phenomena.

Recommendation (5): TEACC can be applied to complex and dynamic distortion to address real-world issues. With the radial and circumferential transfer of mass and momentum, complex total pressure inlet distortion patterns can be investigated to determine their effects on compressor performance and operability. The testing community has developed screens which were designed to produce the most severe complex inlet dynamic distortion behavior. Their methodology was based upon steadystate investigations of inlet distortion using the rule of thumb that a distortion pattern which persist less than one rotor revolution has no effect on the compression system. 
TEACC could be used to investigate the flow field before and after severe inlet distortion to determine if inlet dynamic distortion behavior influences the compressor performance to further quantify distortion analysis. 


\section{Bibliography}

Adamczyk, J. J. "Model Equation for Simulating Flows in Multistage Turbomachinery." NASA Technical Memorandum 86869, 1984.

Adamczyk, J. J., Mulac, R. A., and Celestina, M. L. "A Model for Closing the Inviscid Form of the Average-Passage Equation System." NASA Technical Memorandum $87199,1986$.

Adamczyk, J. J., Celestina, M. L., Beach, T. A., and Barnett, M. "Simulation of 3-D Viscous Flow Within a Multi-Stage Turbine." NASA Technical Memorandum 101376, 1989.

Akima, H. "A New Method of Interpolation and Smooth Curve Fitting Based on Local Procedures." Journal of the Association for Computing Machinery, Vol. 17, No. 4, October 1970, pp. 589-602.

Barlett, C. S. and Phares, W. J., "Icing Testing of a Large Full-Scale Inlet at the Arnold Engineering Development Center." AIAA-93-0299. AIAA $31^{\text {st }}$ Aerospace Science Meeting \& Exhibit, Reno, NV, January 1993.

Beale, D. K. and Kelly, P. G. "Subscale Validation of a Freejet Inlet-Engine Test Capability." AIAA-93-2179. AIAA/SAE/ASME/ASEE 29" Joint Propulsion Conference and Exhibit, Monterey, CA, June 1993.

Beam, R. and Warming, R. F. "An Implicit Finite Difference Algorithm for Hyperbolic Systems in Conservation Law Form." Journal of Computational Physics, Vol. 22, No. 1, September 1976, pp. 87-110.

Billet, G., Laval, P., and Chevalier, P. "Response of an Axial Compressor to Distorted Inlet Flow.” Conference on Computational Methods in Turbomachinery, Birmingham, AL, April 1984.

Billet, G., Laval, P., and Chevalier, P. "Numerical Simulation of the Response of an Axial Compressor to an Nonhomogeneous Flow." La Recherche Aerospatiale, 1985-4, pp. 19-32.

Billet, G., Huard, J., Chevalier, P., and Laval, P. "Experimental and Numerical Study of the Response of an Axial Compressor to Distorted Inlet Flow." Transactions of the ASME, Journal of Fluids Engineering, Vol. 110, December 1988, pp. 355360. 
Bouillet, R. and Brasseur, J. M. "Sources of Distortion and Compatibility." AGARD Lecture Series, No. 72, November 1974, pp. 1-11.

Bry, P., Laval, P., and Billet, G. "Distorted Flow Field in Compressor Inlet Channels." ASME-82-GT-125. Transactions of the ASME, Journal of Engineering for Gas Turbine and Power, Vol. 107, July 1985, pp. 782-791.

Callahan, G. M. and Stenning, A. H. "Attenuation of Inlet Flow Distortion Upstream of Axial Flow Compressors." Journal of Aircraft, Vol. 8, No. 4, April 1971, pp.227233.

Cetin, M., Ucer, A. S., Hirsch, C., and Serovy, G. K. "Application of Modified Loss and Deviation Correlations to Transonic Axial Compressors.” AGARD-R-745. 1987.

Chalk, J. C. H. "Adaptation of a Three-dimensional Numerical Simulation to Represent Gas Turbine Engine Compression Systems." MS Thesis, The University of Tennessee, Knoxville, TN, 1996.

Chima, R. V. and Yokota, J. W. "Numerical Analysis of Three-dimensional Viscous Internal Flows." NASA-TM-100878. First National Fluid Dynamics Congress, Cincinnati, OH, July 1988.

Cohen, H., Rogers, G. F. C., and Saravanamuttoo, H.I.H. Gas Turbine Theory. Longman Scientific \& Technical, England, 1987 (Third Edition).

Colpin, J. "Propagation of Inlet Flow Distortions Through an Axial Compressor Stage." Transactions of the ASME, Journal of Engineering for Power, Vol. 101, January 1979, pp.116-124.

Cooper, G. K. and Sirbaugh, J. R. "PARC Code: Theory and Usage." AEDC-TR89-15. December 1989.

Copenhaver, W. W., Hah, C., and Puterbaugh, S. L. "Three-dimensional Flow Phenomena in a Transonic, High-Through-Flow, Axial-Flow Compressor Stage." ASME-92-GT-169. International Gas Turbine and Aeroengine Congress and Exposition, Cologne, Germany, June 1992.

Crook, A. J. et al. "Numerical Simulation of Compressor Endwall and Casing Treatment Flow Phenomena." ASME-92-GT-300. International Gas Turbine and Aeroengine Congress and Exposition, Cologne, Germany, June 1992. 
Cumpsty, N. A. Compressor Aerodynamics. Longman Scientific \& Technical, England, 1989.

Davis, M. W., Jr. "A Stage-by-Stage Post-Stall Compression System Modeling Technique: Methodology, Validation, and Application." Ph.D. Dissertation, Virginia Polytechnic Institute and State University, Blacksburg, VA, 1986.

Davis, M. W., Jr. "Parametric Investigation into the Combined Effects of Pressure and Temperature Distortion on Compression System Stability." AIAA-91-1895. $27^{\text {th }}$ AIAA/SAE/ASME/ASEE Joint Propulsion Conference, Sacramento, CA, June 1991.

Dawes, W. N. "Computation of Off-Design Flows in a Transonic Compressor Rotor." ASME 85-GT-1. 30 International Gas Turbine Conference and Exhibit, Houston, TX, March 1985.

Dawes, W. N. "Toward Improved Throughflow Capability: The Use of Threedimensional Viscous Flow Solvers in a Multistage Environment." Transactions of the ASME, Journal of Turbomachinery, Vol. 114, January 1992, pp. 8-17.

Dawes, W. N. "The Simulation of Three-dimensional Viscous Flow in Turbomachinery Geometries Using a Solution-Adaptive Unstructured Mesh Methodology." Transactions of the ASME, Journal of Turbomachinery, Vol. 114, July 1992, pp. 528-537.

Denton, J. D. "The Calculation of Three-dimensional Viscous Flow Through Multistage Turbomachines." Transactions of the ASME, Journal of Turbomachinery, Vol. 114, January 1992, pp. 18-26.

Ehrich, F. "Circumferential Inlet Distortions in Axial Flow Turbomachinery." Journal of Aeronautical Sciences, Vol. 24, June 1957, pp. 413-417.

Fuhs, A. E. "Introduction to Distortion Induced Engine Instability." AGARD Lecture Series, No. 72, November 1974.

Garrard, G. D. "Calibration of an Axisymetrical Navier-Stokes Computational Fluid Dynamics Program for Predicting Steady State Diffuser Performance." MS Thesis, The University of Tennessee, Knoxville, TN, 1990.

Garrard, G. D. "ATEC: The Aerodynamic Turbine Engine Code for the Analysis of Transient and Dynamic Gas Turbine Engine System Operations." Ph.D.

Dissertation, The University of Tennessee, Knoxville, TN, 1995. 
Giles, M. B. "Stator/Rotor Interaction in a Transonic Turbine." AIAA-88-3093.

AIAA/SAE/ASME/ASEE Joint Propulsion Conference, Boston, MA, July 1988.

Goethert, B. H. and Reddy, K. C. "Unsteady Aerodynamics of Rotor Blades of a Compressor Under Distorted Flow Conditions." AGARD Fluid Dynamics Specialist Meeting on Aerodynamic Interference, Silver Springs, MD, September 1970.

Gostelow, J. P., Krabacher, K. W, and Smith, L. H., Jr. "Performance Comparisons of High Mach Number Compressor Rotor Blading." NASA CR-1256. December 1968.

Greitzer, E. M. and Strand, T. "Asymmetric Swirling Flows in Turbomachine Annuli." ASME-78-GT-109. ASME Gas Turbine Conference, London, England, April 1978.

Hah, C. "Calculation of Three-dimensional Viscous Flows in Turbomachinery with an Implicit Relaxation Method." Journal of Propulsion, Vol. 3, No. 5, SeptemberOctober 1987, pp. 415-422.

Hale, A. A. and Davis, M. W., Jr. "DYNamic Turbine Engine Compressor Code, DYNTECC - Theory and Capabilities." AIAA-92-3190. AIAA/SAE/ASME/ASEE $28^{\text {th }}$ Joint Propulsion Conference, Nashville, TN, July 1992.

Hale, A. A., Davis, M. W., Jr., and Kneile, K. R. "Turbine Engine Analysis Compressor Code: TEACC, Part I: Technical Approach and Steady Results." AIAA-94-0148. $32^{\text {nd }}$ Aerospace Sciences Meeting, Reno, NV, January 1994.

Hawthorne, W. R. "The Growth of Secondary Circulation in Frictionless Flow." Proc. Cambridge Philosophical Society, Vol. 51, Part 4, 1955, pp. 737-743.

Hearsey, R. M. "HT0300 - A Computer Program for the Design and Analysis of Axial Turbomachinery." Cambridge, MA, March 1970.

Hirsch, C. Numerical Computation of Internal and External Flows: Volume 2:

Computational Methods for Inviscid and Viscous Flows. John Wiley \& Sons Ltd., England, 1990.

Hoffmann, K. and Chiang, S. Computational Fluid Dynamics for Engineers-Volume I. Engineering Education System, Kansas, 1993. 
Hoffmann, K. and Chiang, S. Computational Fluid Dynamics for Engineers- Volume II. Engineering Education System, Kansas, 1993.

Hoffmann, K., Chiang, S., Siddiqui, S., and Papadakis, M. Fundamental Equations of Fluid Mechanics. Engineering Education System, Kansas, 1996.

Horlock, J. H. Axial Flow Compressors: Fluid Mechanics and Thermodynamics. Robert E. Krieger Publishing, Malabar, FL, 1958.

Huard, J. and Werle, H. "Influence d'une Distorsion Amont sur l'Ecoulement dans une Grille d'Aubes Annulaire Fixe." Entropie, No. 81, maijuin 1978, pp. 15-20.

Jameson, A., Schmidt, W., and Turkel, E. "Numerical Solutions of the Euler Equations by Finite Volume Methods Using Runge-Kutta Time-Stepping Schemes." AIAA81-1259. AIAA $14^{\text {th }}$ Fluid and Plasma Dynamics Conference, Palo Alto, CA, 1981.

Jennions, I. K. and Stow, P. "A Quasi-Three-Dimensional Turbomachinery Blade Design System: Part I- Throughflow Analysis." Journal of Engineering for Gas Turbines and Power, Vol. 107, April 1985, pp. 301-316.

Johnsen, I. A. and Bullock, R. O., editors, Aerodynamic Design of Axial-Flow Compressors. NASA-SP-36. National Aeronautics and Space Administration, Lewis Research Center, 1965.

Jones, III, R. R., Heikkinen, B. D., and Abdelwahab, M., "S/MTD Exhaust Gas Management System CFD Analysis." AIAA-94-3214, AIAA/ASME/ASE/ASEE 30th Joint Propulsion Conference, June 1994.

Jorgenson, P. C. E. and Chima, R. V. "An Explicit Runge-Kutta Method for Unsteady Rotor/ Stator Interaction." AIAA-88-0049, 26 $6^{\text {th }}$ AIAA Aerospace Sciences Meeting, Reno, NV, January 1988.

Jorgenson, P. C. E. and Chima, R. V. "An Unconditionally Stable Runge-Kutta Method for Unsteady Flows." AIAA-89-0205, 1989. 27 $7^{\text {th }}$ Aerospace Sciences Meeting, Reno, NV, January 1989.

Joubert, H. "Flowfield Calculation in Compressor Operating with Distorted Inlet Flow." ASME-90-GT-212. Gas Turbine and Aeroengine Congress and Exposition, Brussels, Belgium, June 1990. 
Kimzey, W. F. "An Analysis of the Influence of Some External Disturbances on the Aerodynamic Stability of Turbine Engine Axial Flow Fans and Compressors." AEDC-TR-77-80. August 1977.

Korn, J. A. "Estimated Effect of Circumferential Distortion on Axial Compressors Using Parallel Compressor Theory and Dynamic Stall Delay." AIAA-74-233. AIAA $12^{\text {th }}$ Aerospace Sciences Meeting, Washington, D. C., January 1974.

Lindau, J. W. V. "Multidimensional Dynamic Compression System Modeling." Ph.D Thesis, Virginia Polytechnic Institute and State University, Blacksburg, VA, 1995.

Longley, J. P. "Measured and Predicted Effects of Inlet Distortion on Axial Compressors." ASME-90-GT-214. Gas Turbine and Aeroengine Congress and Exposition, Brussels, Belgium, June 1990.

Longley, J. P. and Greitzer, E. M. "Inlet Distortion Effects in Aircraft Propulsion System Integration." AGARD-LS-183. Advisory Group for Aerospace Research \& Development Lecture Series, May 1992.

Mazzawy, R. S. and Banks, G. A. "Modeling and Analysis of the TF30-P-3 Compressor System with Inlet Pressure Distortion." NASA-CR-134996. April 1976.

Mazzawy, R. S. and Banks, G. A. "Circumferential Distortion Modeling of the TF30-P-3 Compression System." NASA-CR-135124. January 1977.

Mazzawy, R. S. "Multiple Segment Parallel Compressor Model for Circumferential Flow Distortion." Transactions of the ASME, Journal of Engineering for Power, April 1977, pp. 288-296.

Mikolajczak, A. A. and Pfeffer, A. M. "Methods to Increase Engine Stability and Tolerance to Distortion." AGARD Lecture Series, No. 72, November 1974.

Moore, F. K. and Greitzer, E. M. "A Theory of Post-Stall Transients in Multistage Axial Compression Systems." NASA Contractor Report 3878, 1985.

Mulac, R. A. and Adamczyk, J. J. "The Numerical Simulation of a High-Speed Axial Flow Compressor." Transactions of the ASME, Journal of Turbomachinery, Vol. 114 , July 1992, pp. 517-527.

$\mathrm{Ni}, \mathrm{R}$. and Bogoian, J. "Prediction of 3-D Multi-Stage Turbine Flow Field Using a Multiple-Grid Euler Solver." AIAA-89-0203. 27 $7^{\text {th }}$ Aerospace Sciences Meeting, Reno, NV, January 1989. 
Novak, R. A. "Streamline Curvature Computing Procedures for Fluid-Flow Problems." Transactions of the ASME, Journal of Engineering for Power, October 1967, pp 478490.

Oates, G. C. Aerothermodynamics of Gas Turbine and Rocket Propulsion. AIAA Education Series, J. S. Przemieniecki, Series Editor-in-Chief, American Institute of Aeronautics and Astronautics, Washington, D. C., 1988, pp. 19-55, 212-216.

Pazur, W. and Fottner, L. "The Influence of Inlet Swirl Distortions on the Performance of a Jet Propulsion Two-Stage Axial Compressor." Transactions of the ASME, Journal of Turbomachinery, Vol. 113, April 1991, pp. 233-240.

Pearson, J. "Wakes in Axial Compressors." Journal of the Royal Aeronautical Society, 63:415-416, July, 1963.

Pinker, R. A. and Herbert, M. V. "Pressure Loss Associated with Compressible Flow Through Square-mesh Wire Gauzes." Journal of Mechanical Engineering Science, Vol. 9, No. 1, 1967, pp. 11-23.

Plourde, G. A. and Stenning, A. H. "Attenuation of Circumferential Inlet Distortion in Multistage Axial Compressors." Journal of Aircraft, Vol. 5, No. 3, May- June 1968, pp. 236-242.

Power, G. D. and Heikkinen, B. D. "CFD Applications in an Aeropropulsion Test Environment.” AIAA-93-1924. 29 ${ }^{\text {th }}$ AIAA/SAE/ASME/ASEE Joint Propulsion Conference and Exhibit, Monterey, CA, June 1993.

Power, G. D. and Cooper, G. K. "NPARC 2.2 - Features and Capabilities." AIAA95-2609. $31^{\text {st }}$ AIAA/ASME/SAE/ASEE Joint Propulsion Conference and Exhibit, San Diego, CA, July 1995.

Power, G. D. and Prufert, M. B. "Computational Evaluation of Diffuser Performance in a Turbine Engine Altitude Test Cell." AIAA-96-0498. 34 AIAA Aerospace Sciences Meeting and Exhibit, Reno, NV, January 1996.

Pulliam, T. H. and Steger, J. L. "Implicit Finite-Difference Simulations of Three Dimensional Compressible Flow." AIAA Journal, Vol. 18, No. 2, February 1980, pp. 159-167. 
Pulliam, T. H. "Euler and Thin Layer Navier-Stokes Codes: ARC2D, ARC3D." Notes for Computational Fluid Dynamics User's Workshop, The University of Tennessee Space Institute, Tullahoma, TN, UTSI Publication E02-4005-023-84, March 12-16, 1984, pp. 15.1-15.85.

Rai, M. M. "Unsteady Three-dimensional Navier-Stokes Simulations of Turbine RotorStator Interaction." AIAA-87-2058. AIAA/SAE/ASME/ASEE $23^{\text {rd Joint }}$ Propulsion Conference, San Diego, CA, June- July 1987.

Rao, K. V. and Delaney, R. A. "Investigation of Unsteady Flow Through a Transonic Turbine Stage: Part 1 - Analysis." AIAA-90-2408. AIAA/SAE/ASME/ASEE $26^{\text {th }}$ Joint Propulsion Conference, Orlando, FL, July 1990.

SAE Aerospace Information Report, AIR-1419, "Inlet Total Pressure Distortion Considerations For Gas Turbine Engines." May 1983.

Sayari, N. and Blocs, A. "A New Throughflow Approach For Transonic Axial Compressor Stage Analysis." ASME-95-GT-195. International Gas Turbine and Aeroengine Congress and Exposition, Houston, TX, June 1995.

Schreiber, H. A. and Starken, H. "An Investigation of a Strong Shock-Wave Turbulent Boundary Layer Interaction in a Supersonic Compressor Cascade." Transactions of the ASME, Journal of Turbomachinery, Vol. 114, July 1992, pp. 494-503.

Seyler, D. R. and Gestolow, J. P. "Single Stage Experimental Evaluation of High Mach Number Compressor Rotor Blading Part 2- Performance of Rotor 1B." NASACR-54582, September 1967.

Shahrokhi, K. A. "Application of Modified Dynamic Compression System Model to a LowAspect Ratio Fan: Effects of Inlet Distortion.” MS Thesis, Mechanical Engineering, Vanderbilt University, Nashville, TN, 1995.

Smith, L. H. "Three-dimensional Flow in Axial-Flow Turbomachinery, Part I: Theoretical Determination of Secondary Flow." Internal Flow Research, Report I-14, Johns Hopkins University, 1953.

Smith, L. H., Jr. "The Radial-Equilibrium Equation of Turbomachinery." Transactions of the ASME, Journal of Engineering for Power, January 1966, pp. 1-12.

Squire, J. B. and Winter, K. G. "The Secondary Flow in a Cascade of Airfoils in a Nonuniform Stream.” Journal of the Aeronautical Sciences, 1951, pp. 271-277. 
Steenken, W. G. "Modeling Compression Component Stability Characteristics - Effects of Inlet Distortion and Fan Bypass Duct Disturbances." AGARD-CP-324. Advisory Group for Aerospace Research \& Development Conference Proceedings, February 1983.

Stenning, A. H. "Inlet Distortion Effects in Axial Compressors." Transactions of the ASME, Journal of Fluids Engineering, Vol. 102, March 1980, pp. 7-13.

Stewart, M. "Axisymmetric Aerodynamic Numerical Analysis of a Turbofan Engine." ASME-95-GT-338. International Gas Turbine and Aeroengine Congress and Exposition, Houston, TX, June 1995.

Subramanian, S. V. "Three-dimensional Multigrid Navier-Stokes Computations for Turbomachinery Applications." AIAA-89-2453. AIAA/ASME/SAE/ASEE $25^{\text {th }}$ Joint Propulsion Conference, Monterey, CA, July 1989.

Tan, C. S. and Greitzer, E. M. "Nonaxisymetric Compressible Swirling Flow in Turbomachinery Annuli." AIAA Journal, Vol. 24, No. 1, 1985, pp. 92-100.

Thompson, J. W. and Abernethy, R. B. "Handbook of Uncertainty in Gas Turbine Measurements." AEDC-TR-73-5, February 1973. 
Appendices 


\section{Appendix A - Tables}

Table 4.1: Variable relaxation factor used to relax the source terms on each axial station through the bladed region by selecting an initial $\phi$ between 1.0 and 0.5 .

\begin{tabular}{|c|c|l|l|l|l|l|l|}
\hline $\begin{array}{c}\text { Axial } \\
\text { Station }\end{array}$ & $\begin{array}{c}\text { Relaxation } \\
\text { Factor }\end{array}$ & $\mathbf{A}$ & \multicolumn{1}{|c|}{ B } & \multicolumn{1}{|c|}{ C } & \multicolumn{1}{|c|}{ D } & \multicolumn{1}{|c|}{ E } & \multicolumn{1}{|c|}{ F } \\
\hline Initial, 1 & $\phi$ & 1.0 & 0.9 & 0.8 & 0.7 & 0.6 & 0.5 \\
\hline 2 & $\phi^{2}$ & 1.0 & 0.81 & 0.64 & 0.49 & 0.36 & 0.25 \\
\hline 3 & $\phi^{3}$ & 1.0 & 0.729 & 0.512 & 0.343 & 0.216 & 0.125 \\
\hline 4 & $\phi^{4}$ & 1.0 & 0.6561 & 0.4096 & 0.2401 & 0.1296 & 0.0625 \\
\hline 5 & $\phi^{5}$ & 1.0 & 0.59049 & 0.32768 & 0.16807 & 0.07776 & 0.03125 \\
\hline 6 & $\phi^{6}$ & 1.0 & 0.531441 & 0.262144 & 0.117649 & 0.046656 & 0.015625 \\
\hline 7 & $\phi^{7}$ & 1.0 & 0.478297 & 0.209715 & 0.082354 & 0.027994 & 0.007813 \\
\hline 8 & $\phi^{8}$ & 1.0 & 0.430467 & 0.167772 & 0.057648 & 0.016796 & 0.003906 \\
\hline 9 & $\phi^{9}$ & 1.0 & 0.38742 & 0.134218 & 0.040354 & 0.010078 & 0.001953 \\
\hline
\end{tabular}

Table 6.1: Validation of clean inlet TEACC with experimental data.

\begin{tabular}{|l|c|c|}
\hline \multicolumn{1}{|c|}{ Variable } & Figure Number & Max. Percent Difference \\
\hline Exit Total Pressure & 6.6 & -1.3362 \\
\hline Total Pressure Ratio & 6.7 & 1.0081 \\
\hline Exit Mach Number & 6.8 & -2.7258 \\
\hline Exit Static Pressure & 6.9 & 1.9213 \\
\hline Exit Total Temperature & 6.10 & 0.9450 \\
\hline Total Temperature Ratio & 6.11 & 1.1345 \\
\hline Exit Static Temperature & 6.12 & 1.8503 \\
\hline Exit Static Density & 6.13 & 1.2508 \\
\hline Exit Absolute Velocity & 6.14 & -3.0563 \\
\hline Exit Circumferential Velocity & 6.15 & -7.7096 \\
\hline
\end{tabular}


Table 7.1: Percent loss in total pressure across screen relative to screen inlet.

\begin{tabular}{|c|c|c|c|c|}
\hline \multicolumn{2}{|c|}{ Description of Screen } & \multicolumn{3}{c|}{ Percent Loss in Total Pressure from Inlet } \\
\hline Screen Type & Screen Blockage & $\mathbf{5 0} \%$ & $\mathbf{7 0 \%}$ & $\mathbf{1 0 0 \%}$ \\
\hline Distortion & 0.5376 & 4.0 & 7.0 & 15.0 \\
\hline Base & 0.1750 & 0.8 & 1.5 & 3.0 \\
\hline
\end{tabular}


Appendix B - Figures 


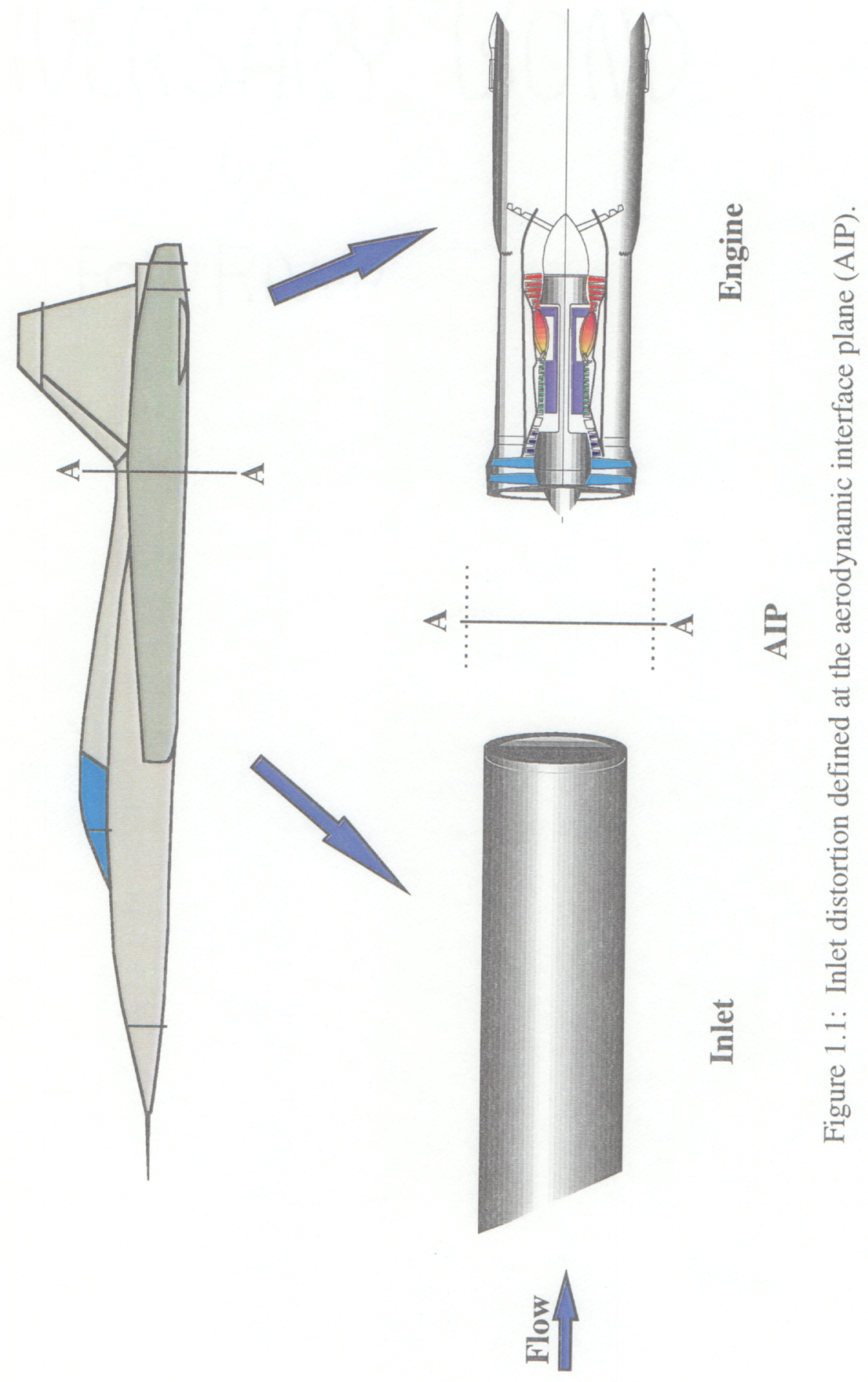




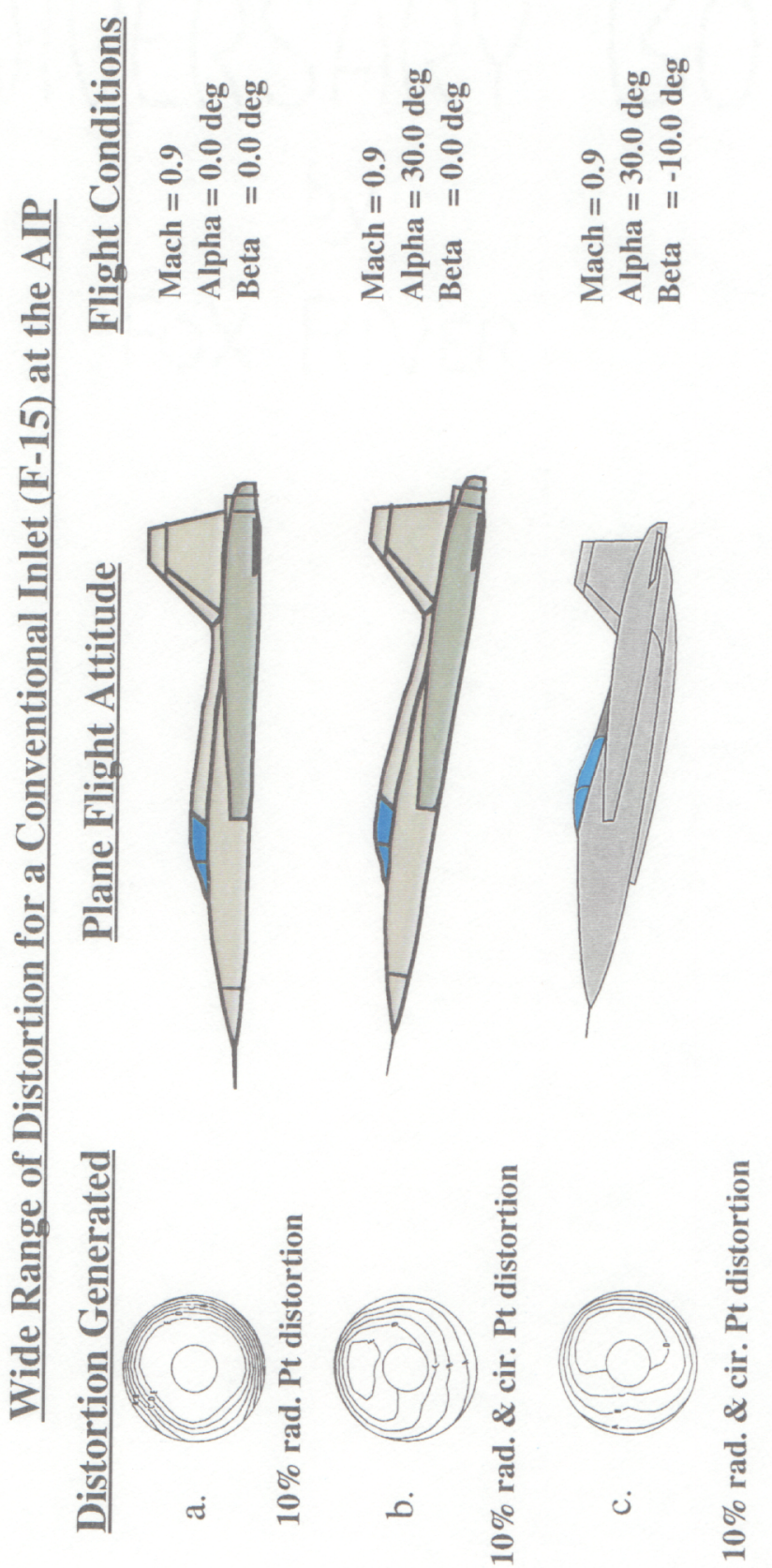

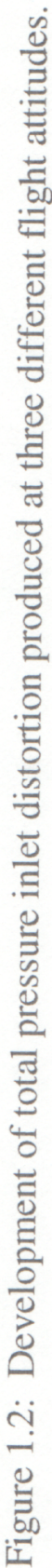




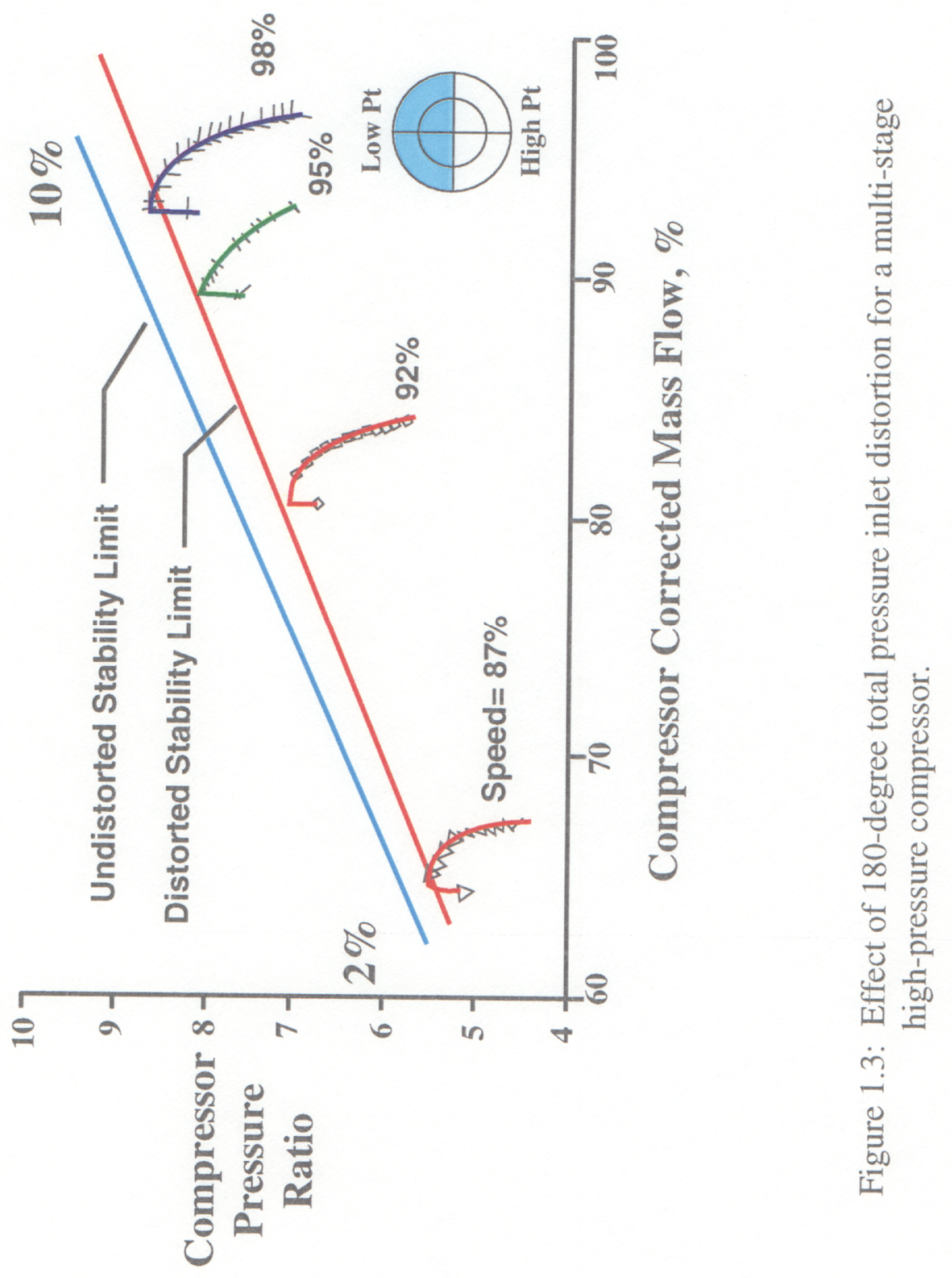




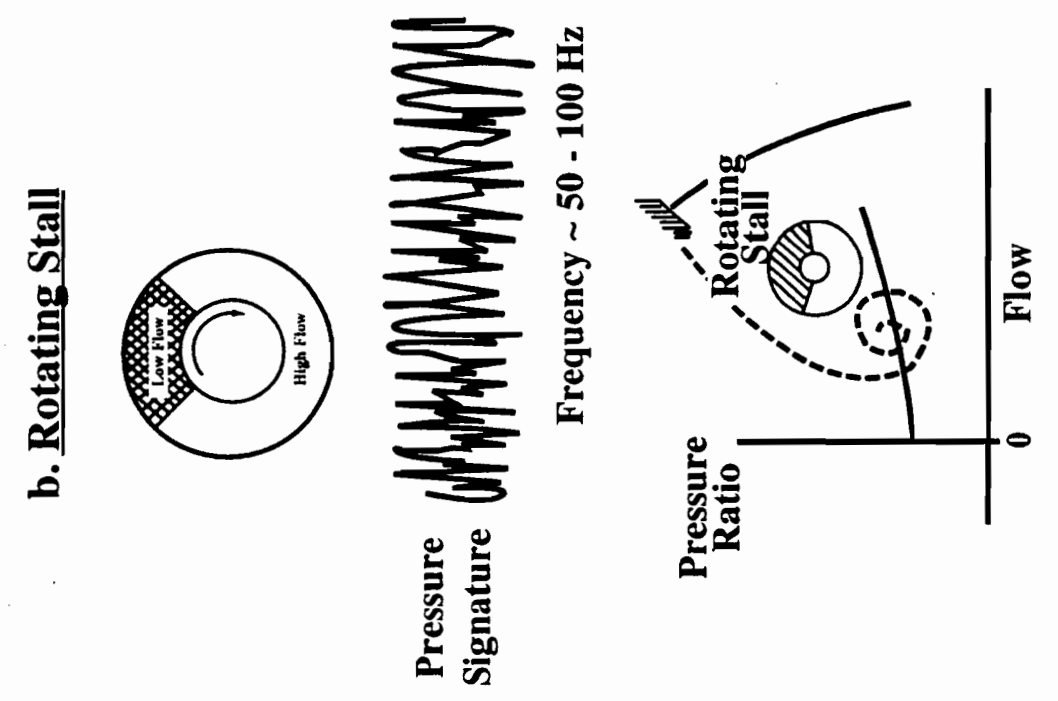

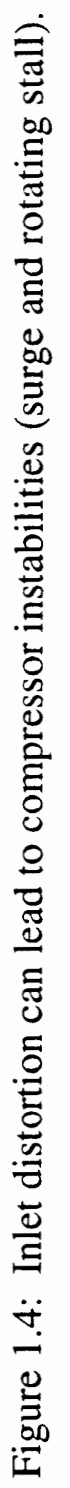




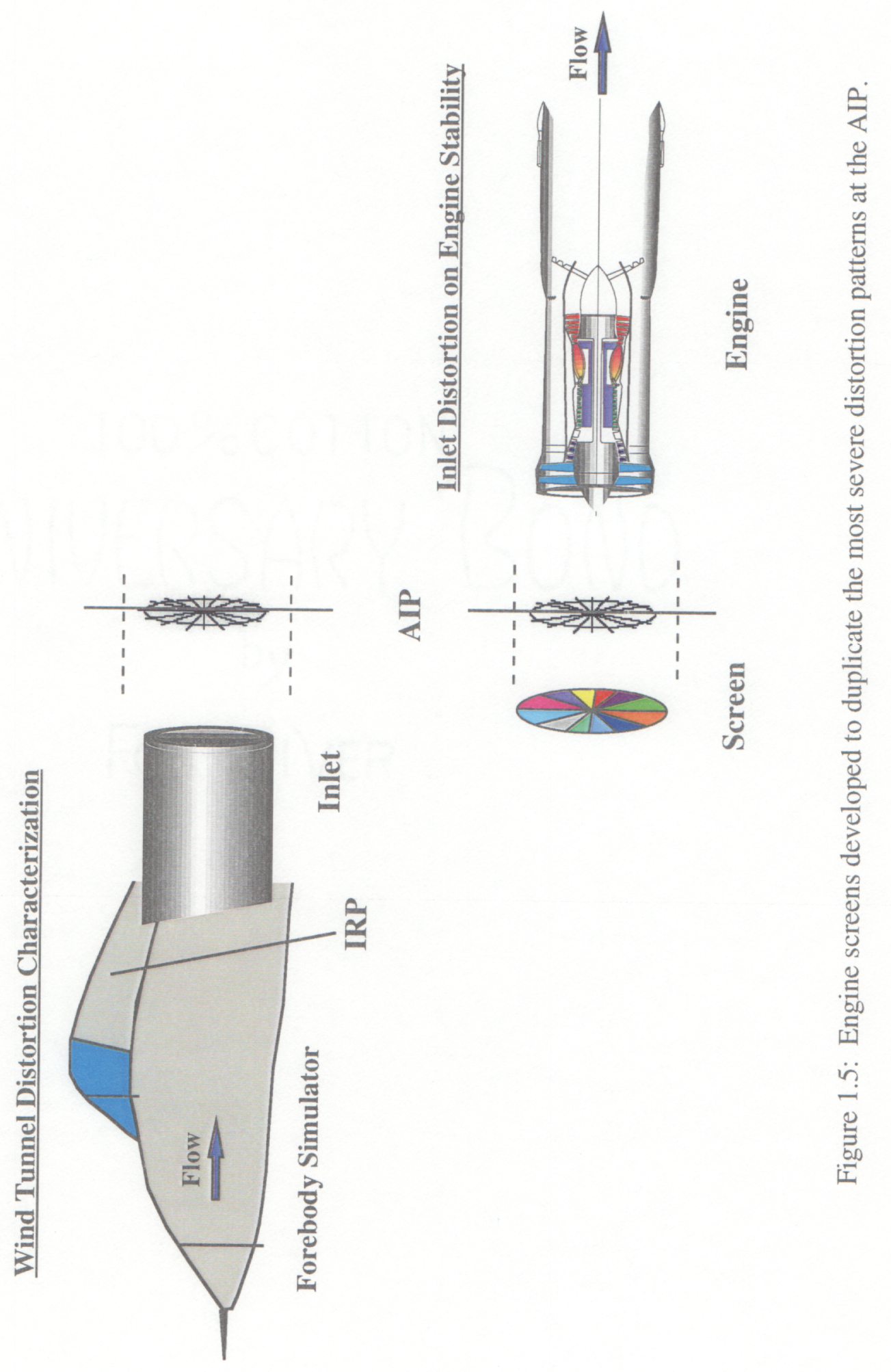




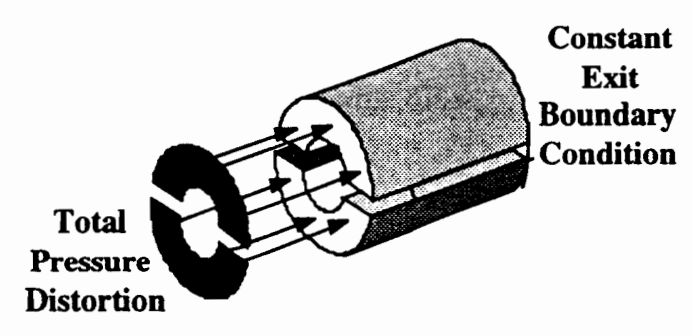

a. Classical parallel theory

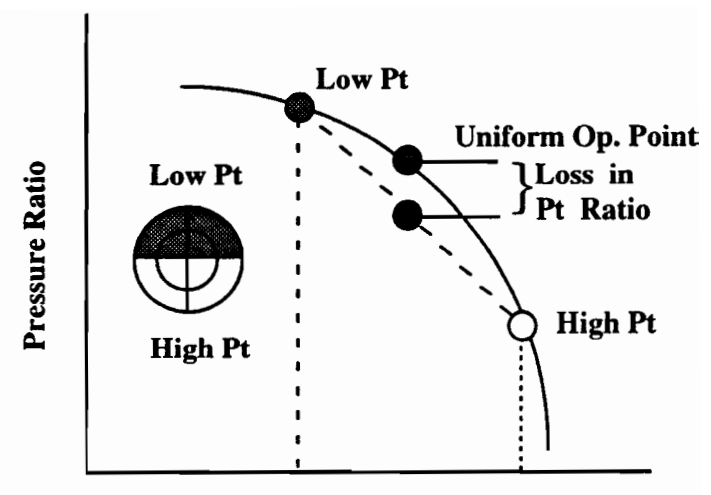

b. Response to total pressure distortion

Figure 2.1: Classical parallel compressor theory with a description of how the two circumferential segments respond to total pressure inlet distortion.

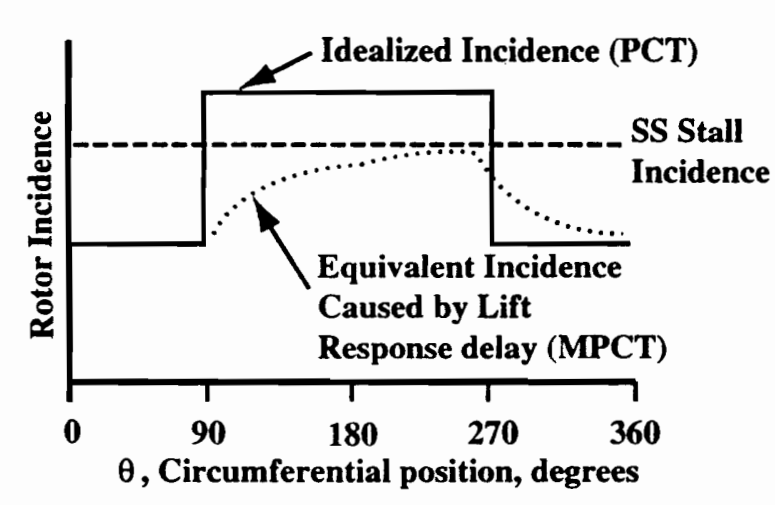

a. Delayed lift response $\quad$ b. Comparison with experimental data

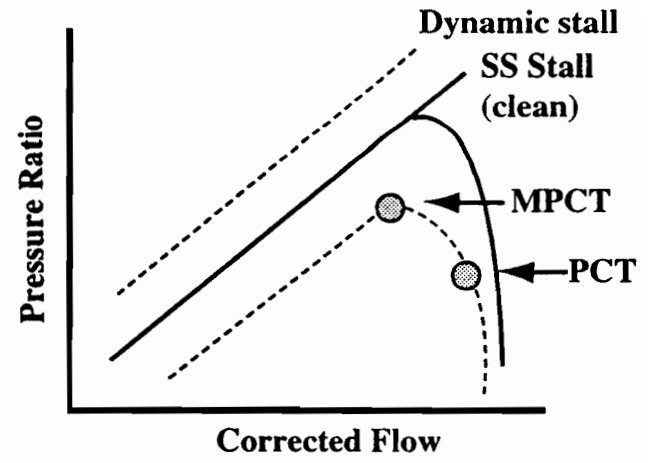

Corrected Flow

Figure 2.2: Delayed lift response for rotors with circumferential inlet distortion (Korn, 1974). 


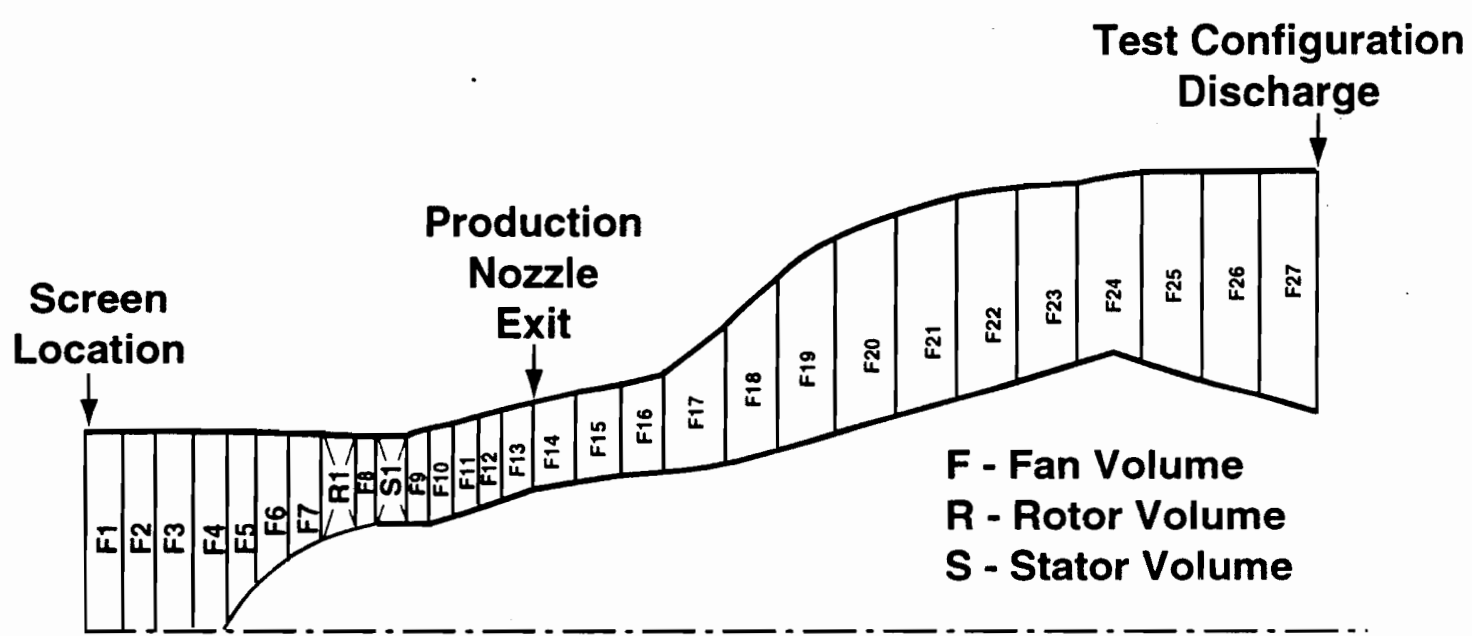

Figure 2.3: Schematic of volumes for circumferential-flow redistribution model (Steenken, 1983).

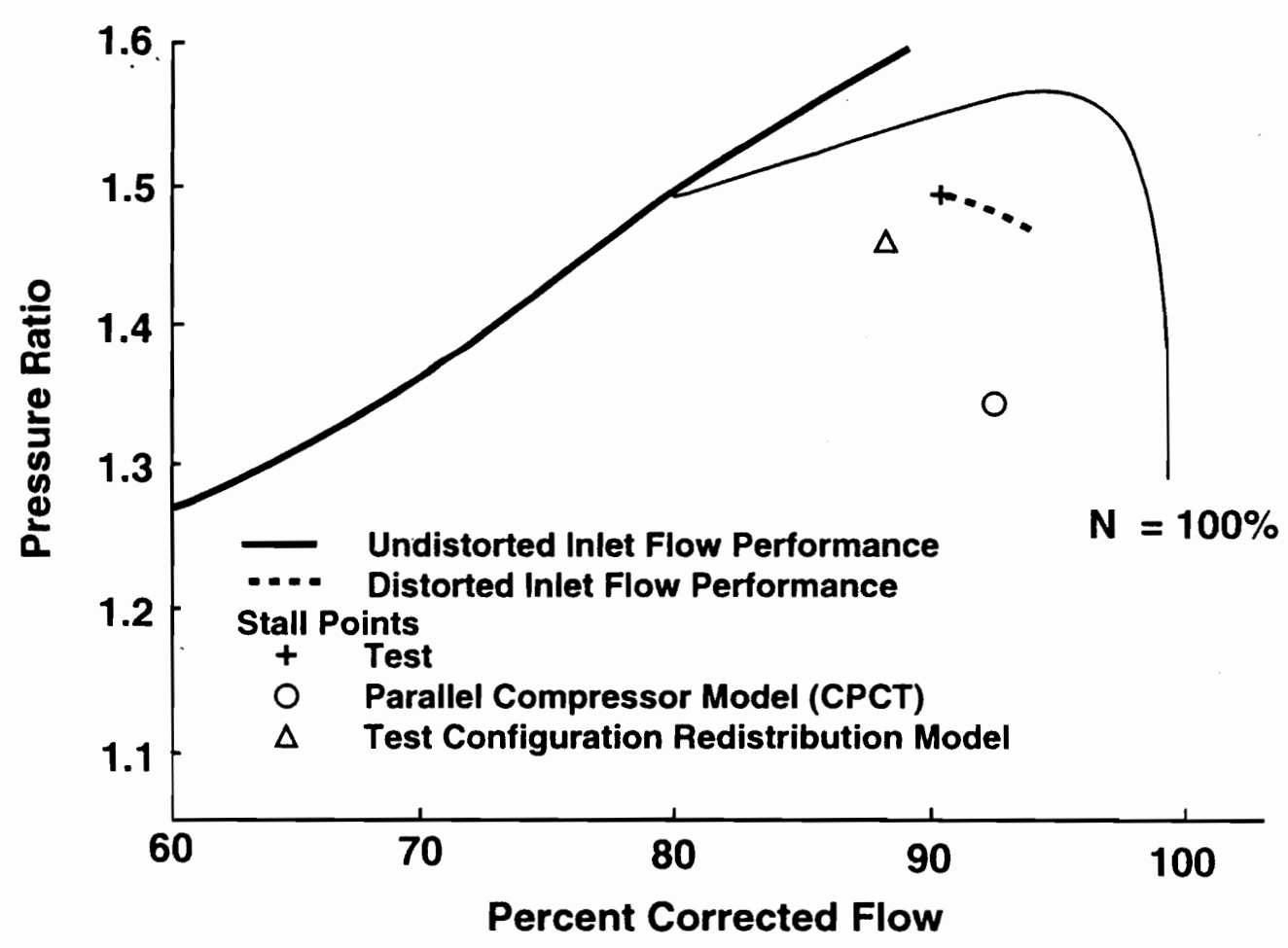

Figure 2.4: Inlet flow distortion simulation results (Steenken, 1983). 


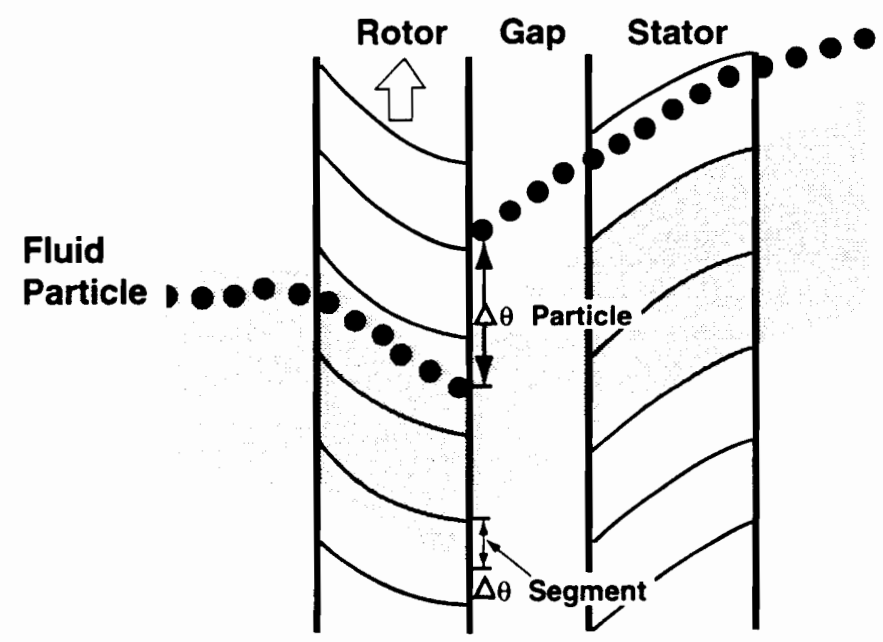

Figure 2.5: Segment and particle circumferential displacement through compressor (Mazzawy, 1977).

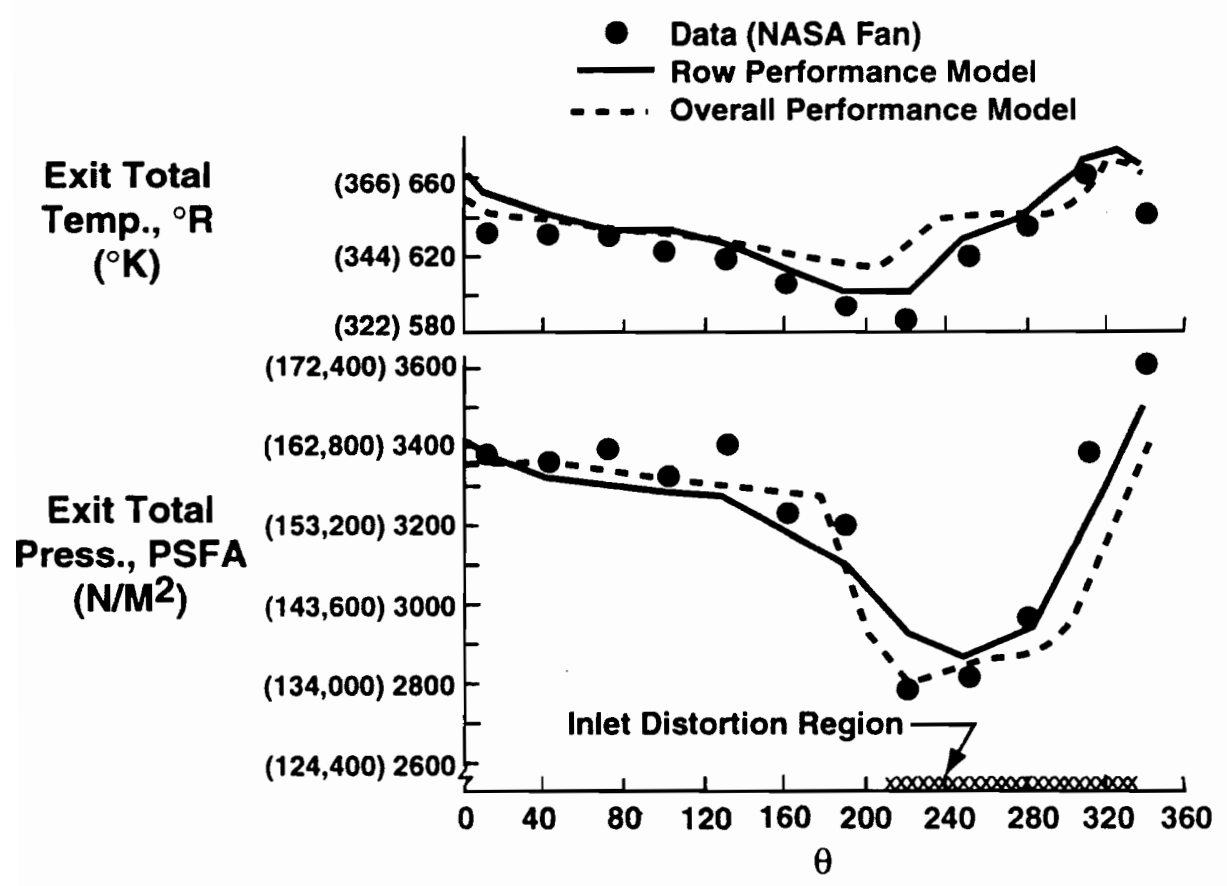

Figure 2.6: Modified multiple-segment model for single-stage fan (Mazzawy, 1977). 

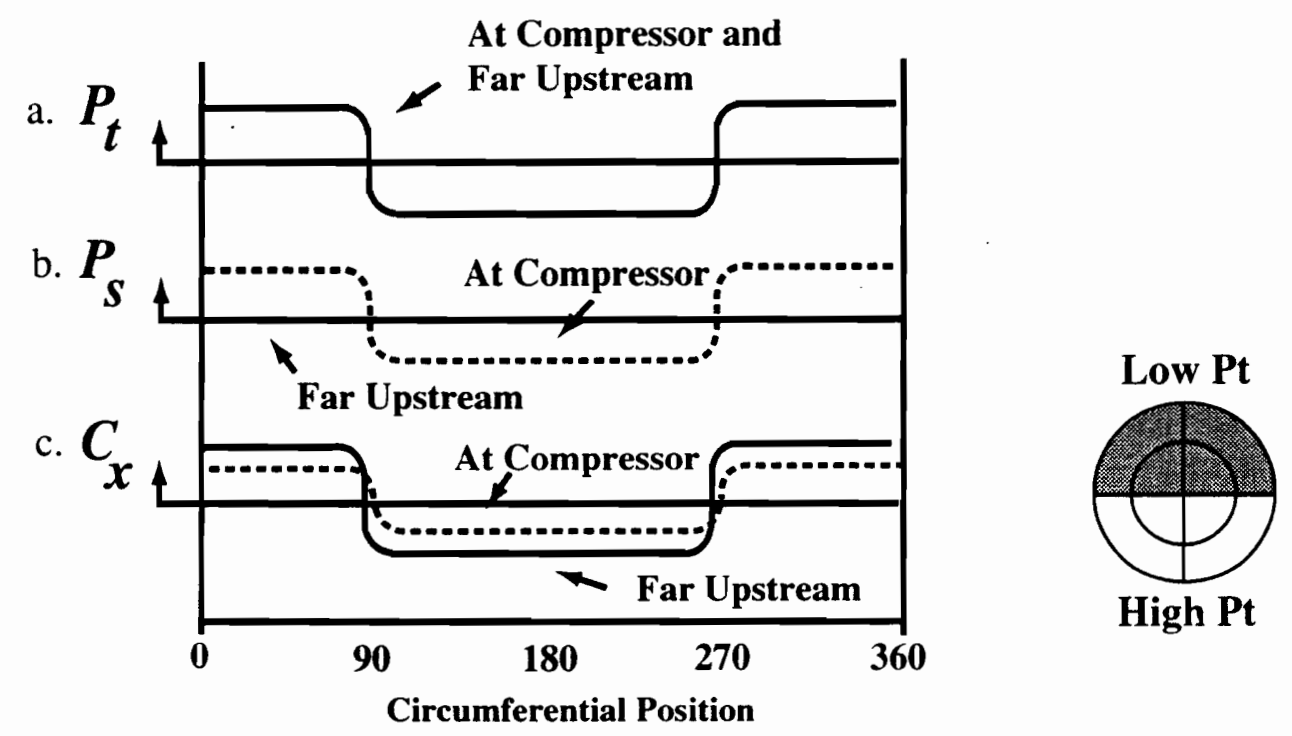

Figure 2.7: Total pressure, static pressure, and axial velocity distributions at a far upstream station and at the compressor inlet (Longley \& Greitzer, 1992).
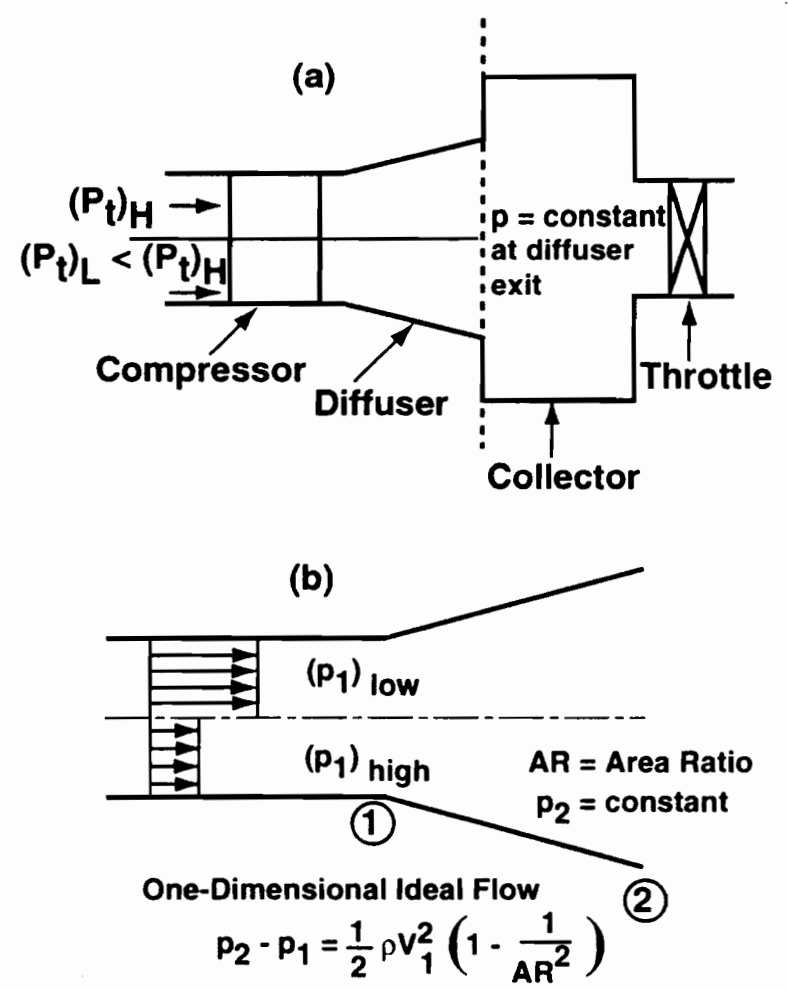

Figure 2.8: Parallel diffuser model for compressor-component coupling effects;

a) parallel diffusers, b) non-uniform flow in a parallel diffuser (Longley \& Greitzer, 1992). 

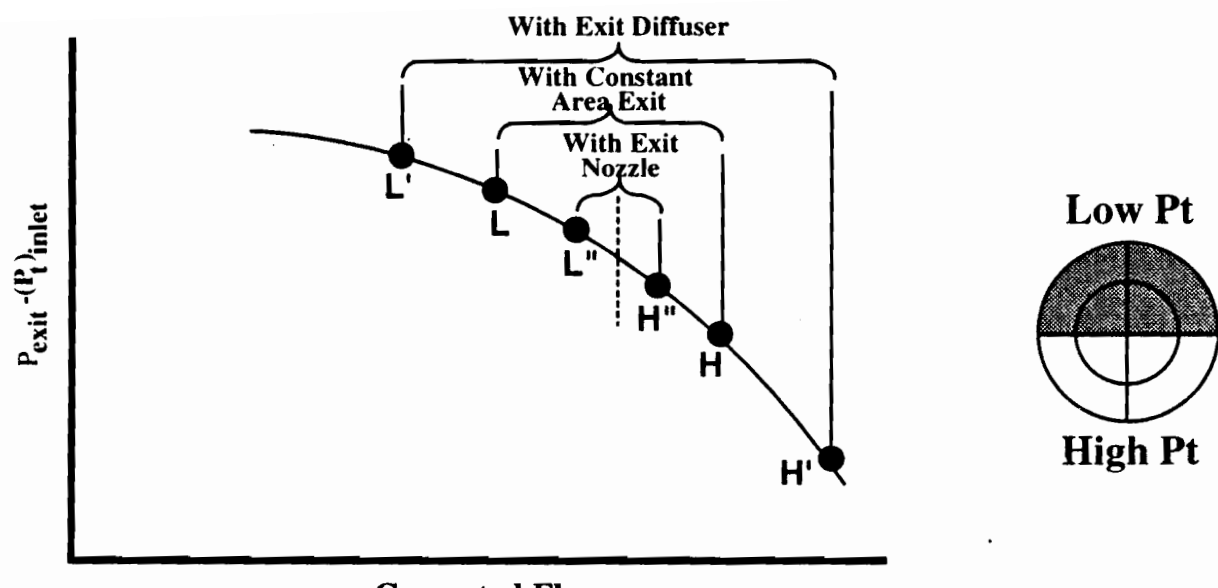

High Pt

Corrected Flow

Figure 2.9: Effect of downstream components on compressor performance with inlet distortion (Longley \& Greitzer, 1992).

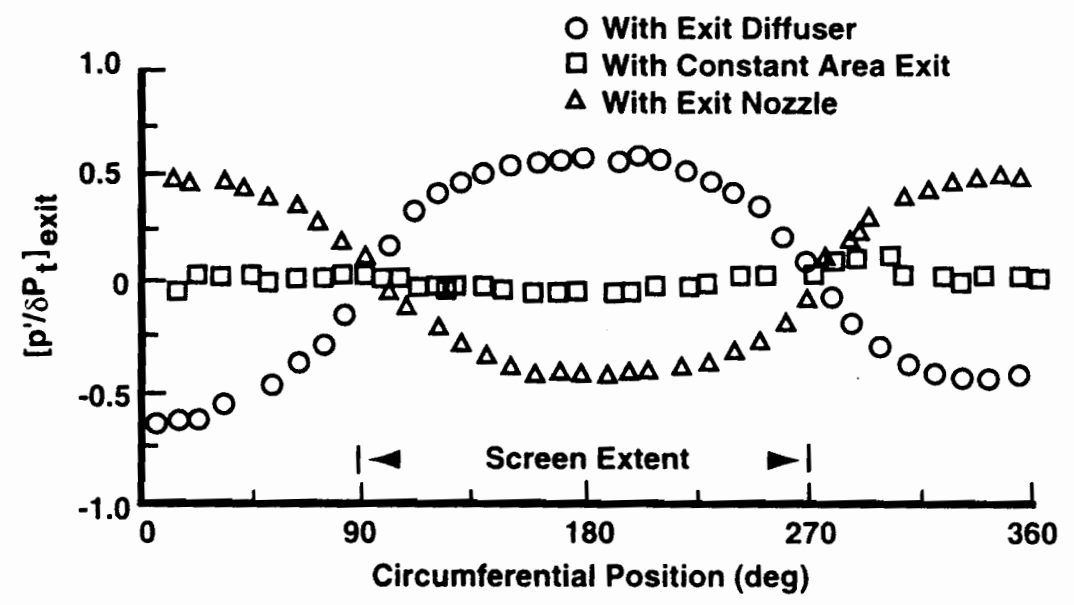

Figure 2.10: Effect of downstream components on static pressure distortion at exit of three-stage compressor (Longley \& Greitzer, 1992). 


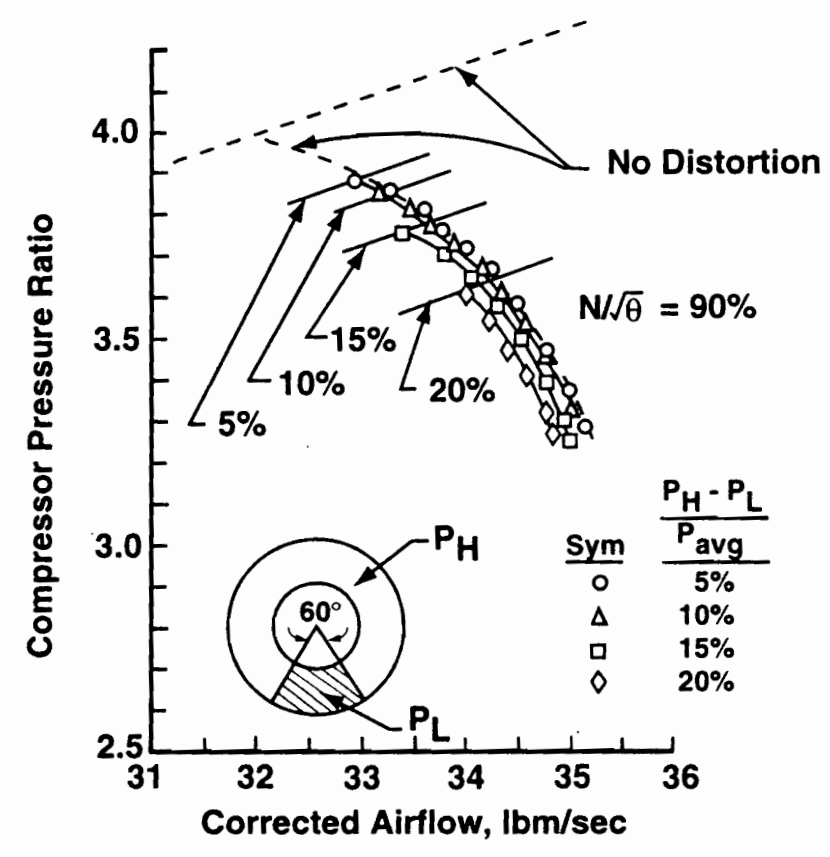

Figure 2.11: Model-computed influence of circumferential pressure distortion on stability, XC-1 compressor (Kimzey, 1977).

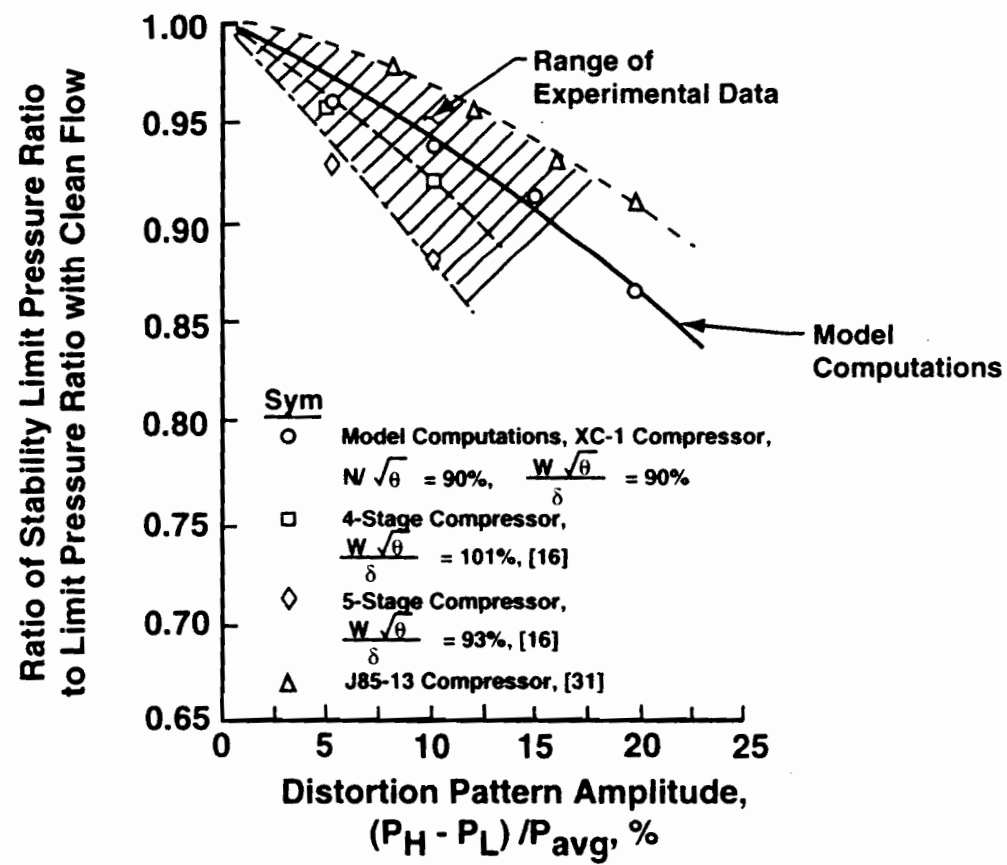

Figure 2.12: Comparison of circumferential distortion model computations with experimental results (Kimzey, 1977). 


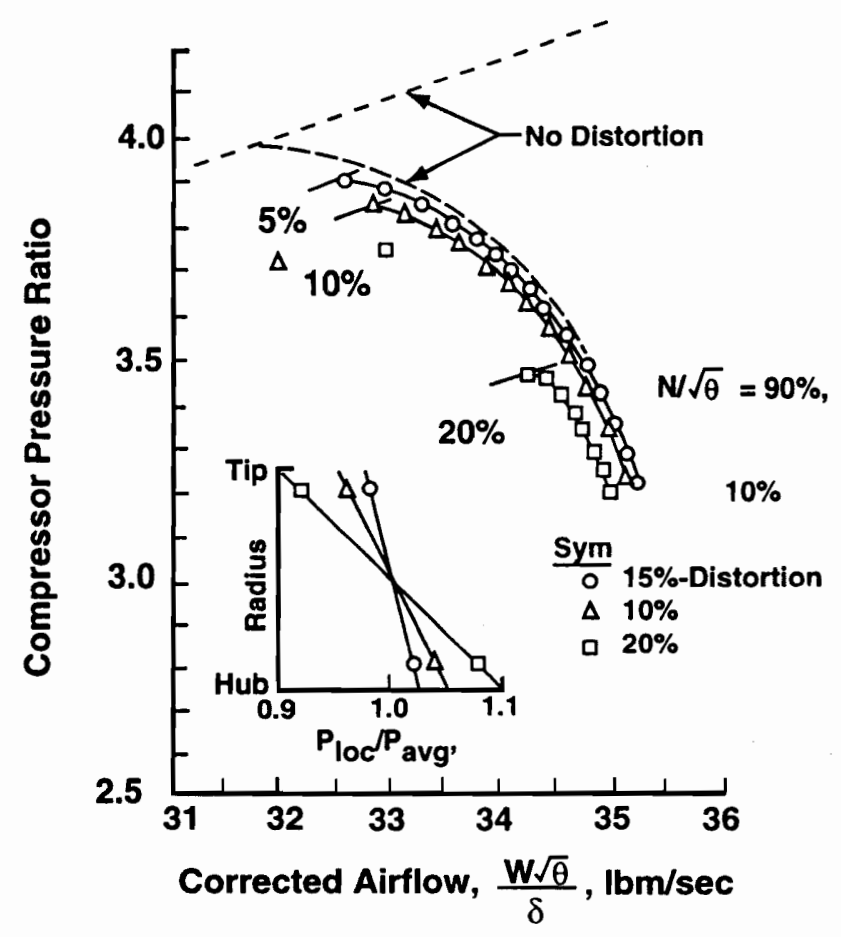

Figure 2.13: Computed radial distortion influences on stability (Kimzey, 1977).

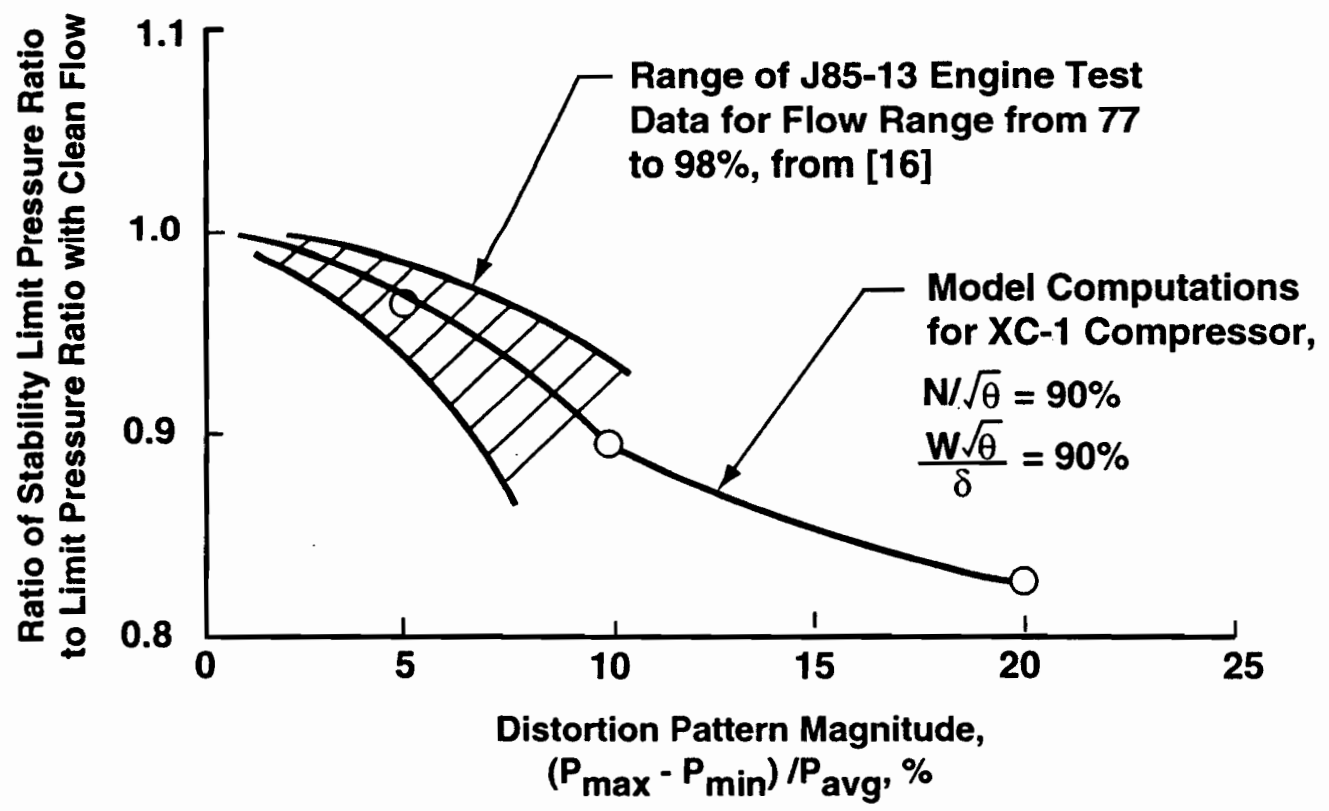

Figure 2.14: Comparison of radial distortion model computations with experimental results (Kimzey, 1977). 


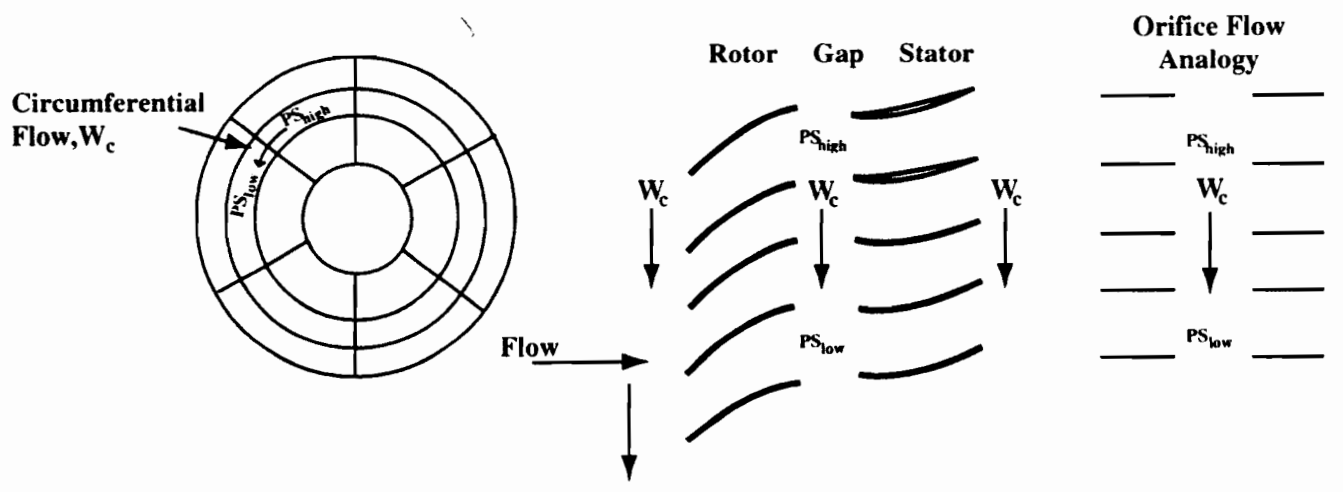

Figure 2.15: Illustration of the circumferential crossflow model with the orifice flow analogy (Shahrokhi, 1995).

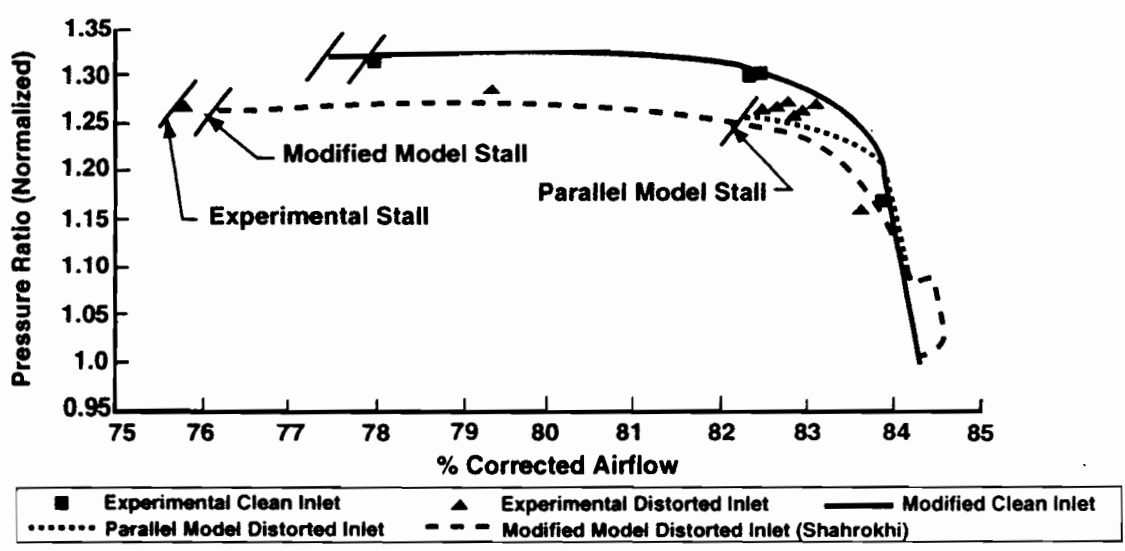

Figure 2.16: Compressor performance prediction for one/revolution circumferential distortion screen at $85 \%$ corrected rotor speed (Shahrokhi, 1995).

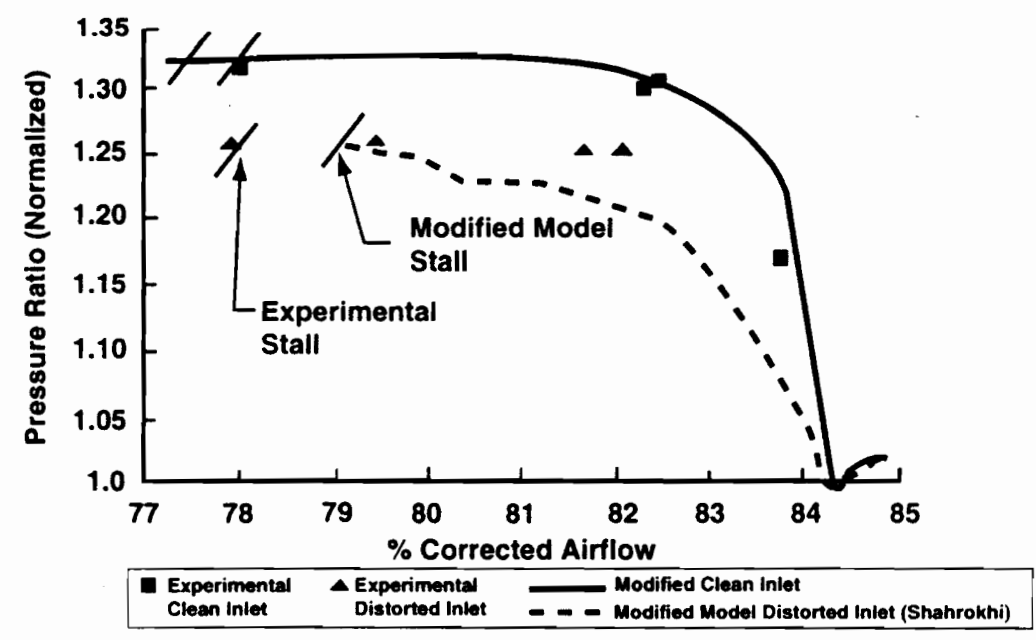

Figure 2.17: Compressor performance prediction for tip-radial distortion screen at $85 \%$ corrected rotor speed (Shahrokhi, 1995). 


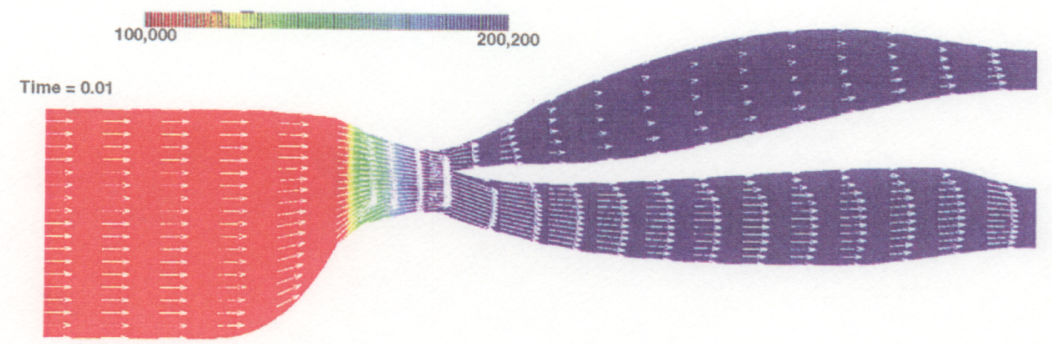

Figure 2.18: First of a sequence of velocity vectors on top of static pressure contours. Simulated fan upstream of splitter. Core exit boundary ramped from Mach $=0.3362$ to $\mathrm{Mach}=0.2($ Lindau, 1995).

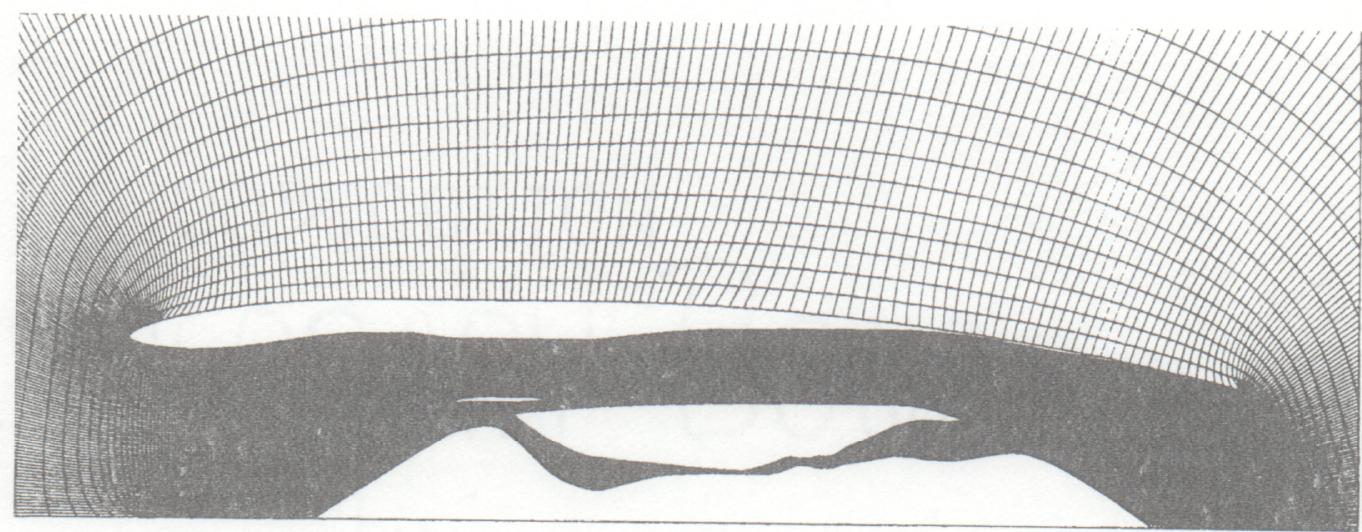

Figure 2.19: Inner region of a multiblock grid for the Energy Efficient Engine simulation. For clarity, every other point is plotted. The entire computational grid contains 90,128 cells in 19 blocks (Stewart, 1995).

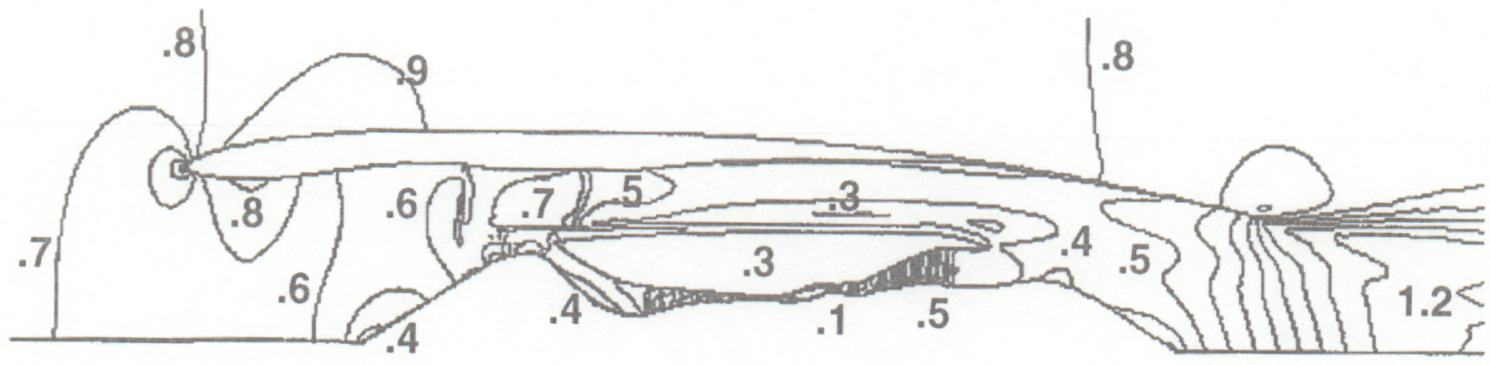

Figure 2.20: Energy Efficient Engine absolute frame Mach contours (in tenths) at the cruise design point, $M_{\infty}=0.8,10.7 \mathrm{~km}$ (Stewart, 1995). 

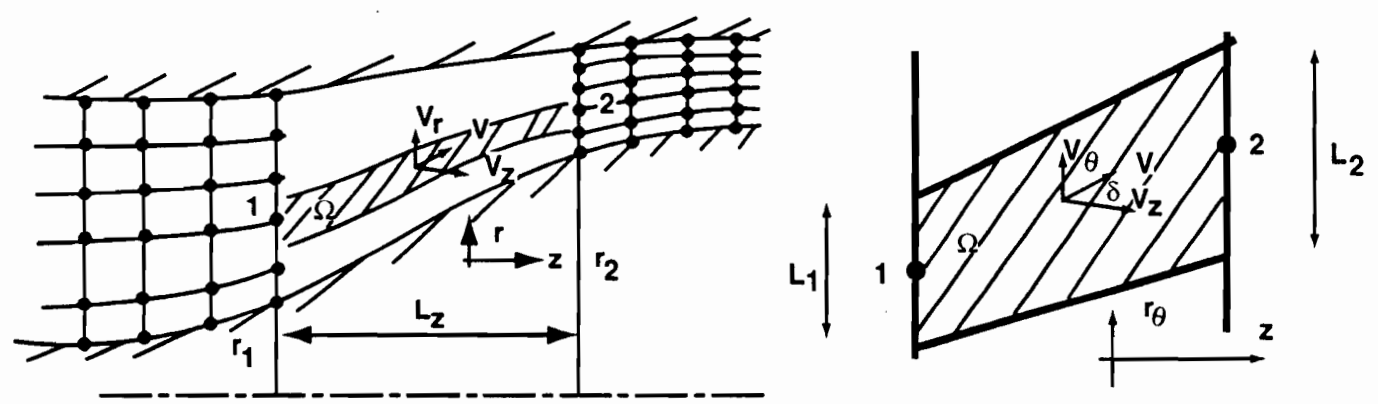

Figure 2.21: Modeling of the blade rows in the physical plane (Billet, 1988).

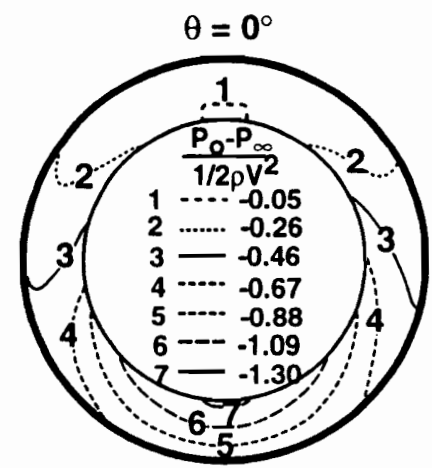

Figure 2.22: Inlet total pressure distribution (Billet, 1988).
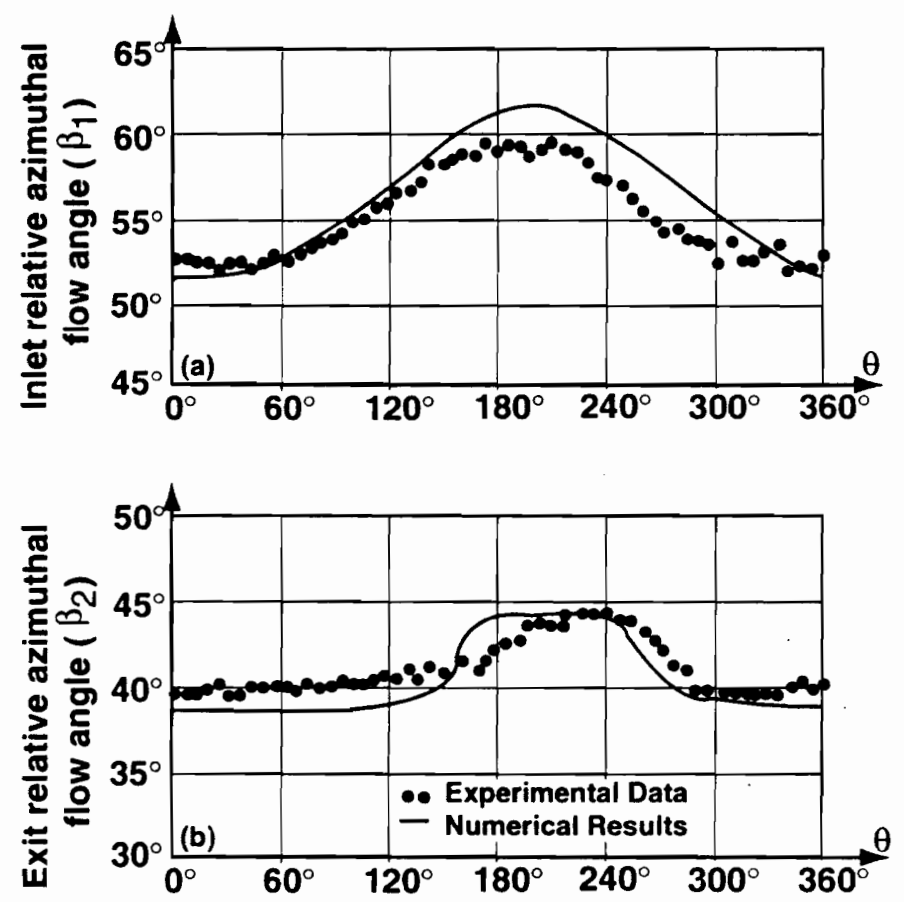

Figure 2.23: Azimuthal flow angle distribution. (a) In front of the rotor: (b) Downstream of the rotor (Billet, 1988). 


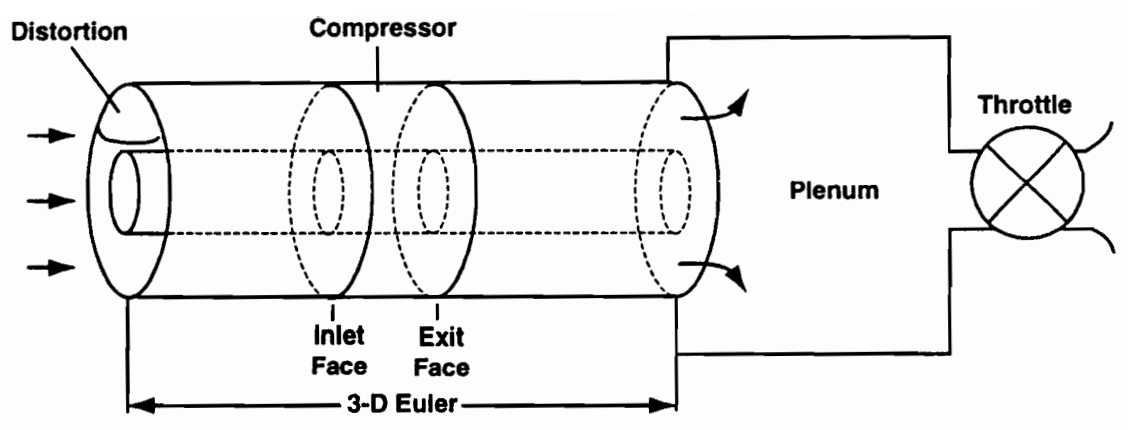

Figure 2.24: The Integrated Flowfield Computational Model (IFCM) for a general compression system model (Tan, 1985).

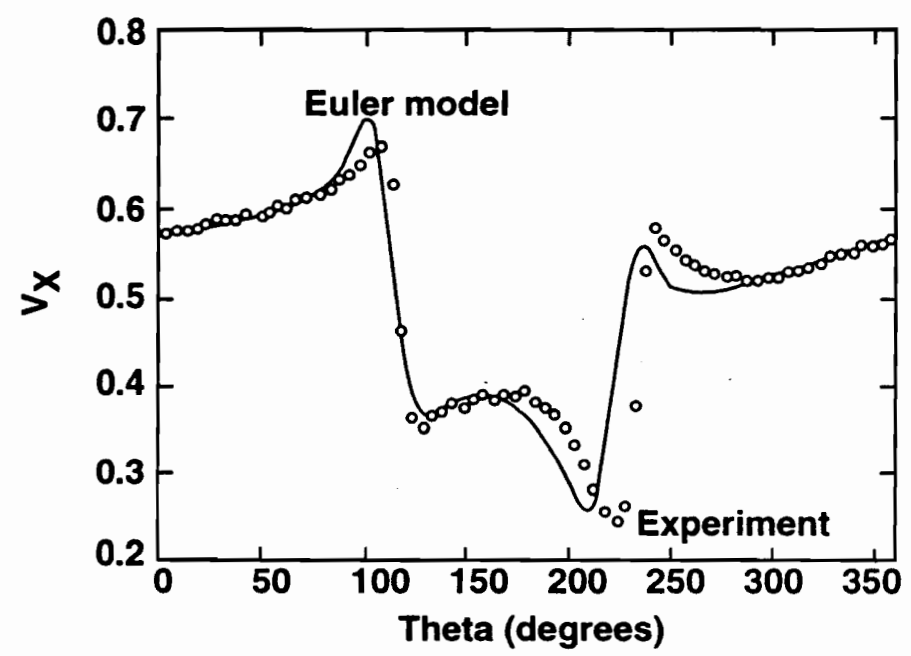

Figure 2.25: Axial velocity at IGV inlet: flow coefficient $=0.5$ (Tan, to be pblished).

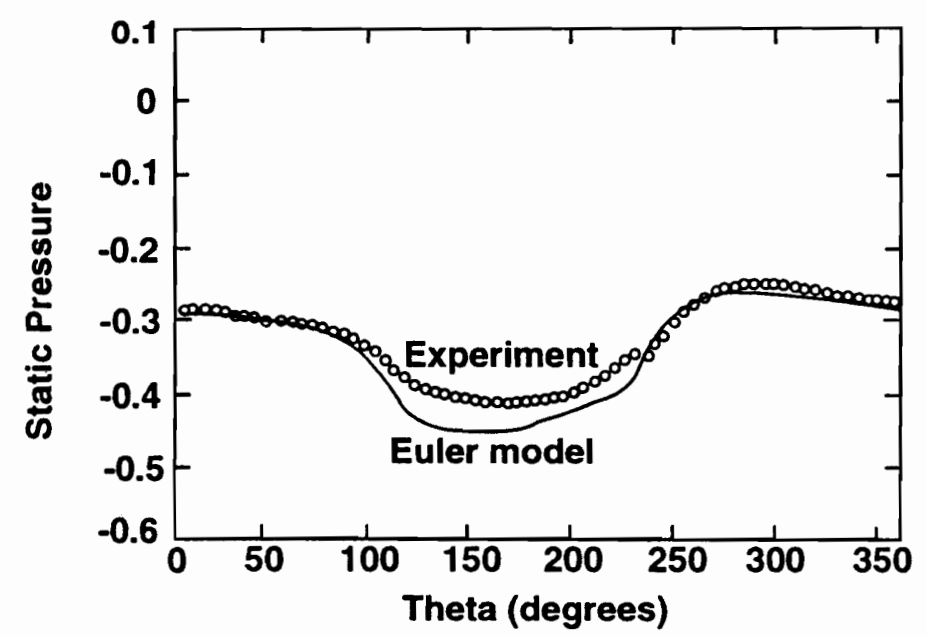

Figure 2.26: Axial velocity at IGV inlet: flow coefficient $=0.5$ (Tan, to be published). 

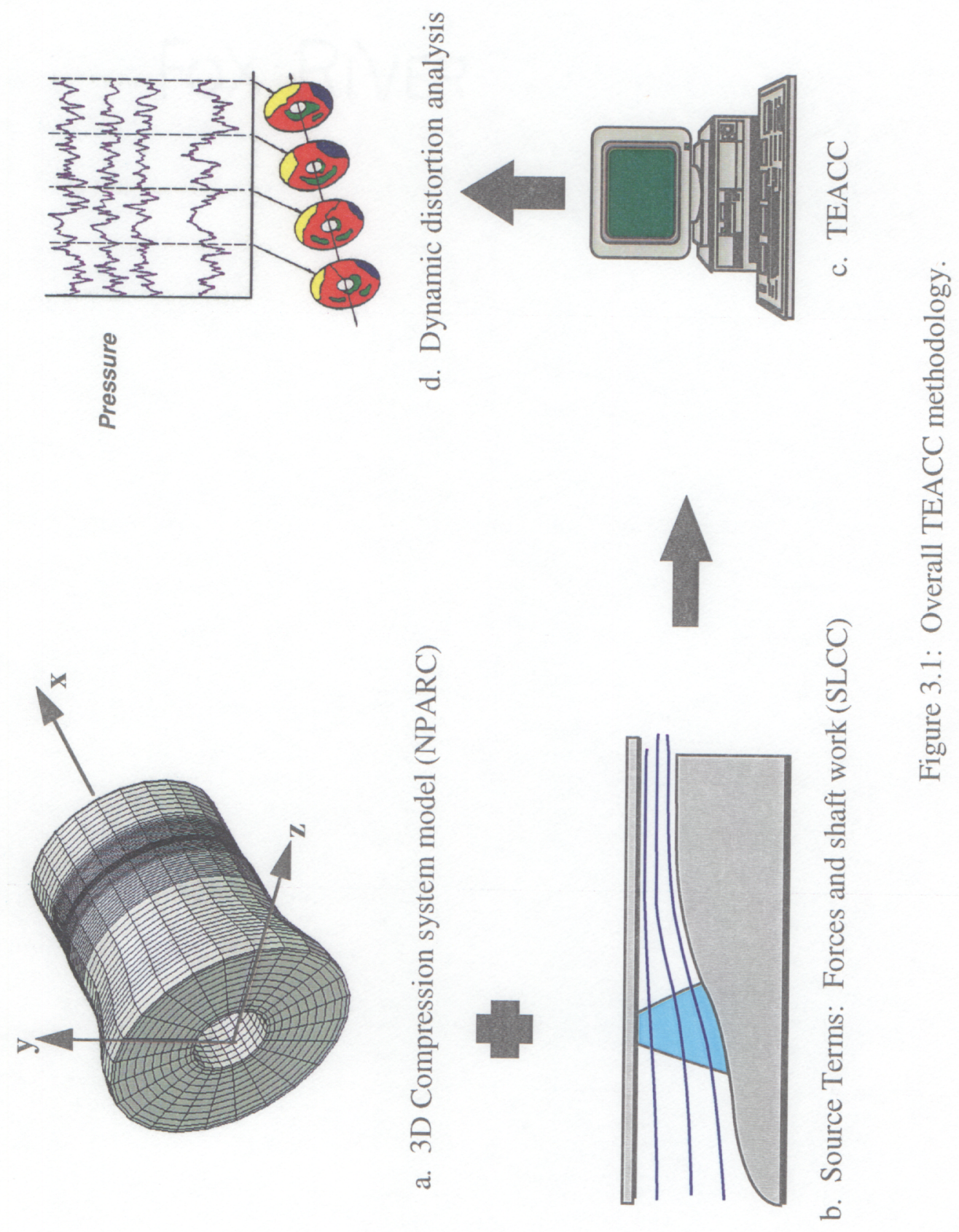


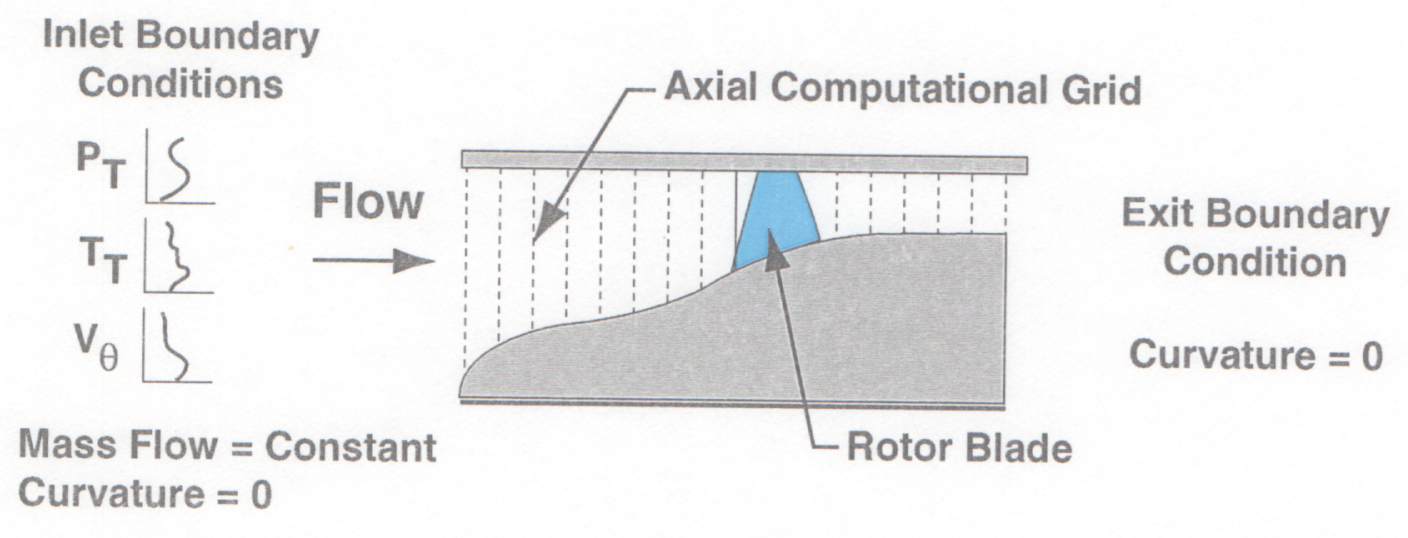

Figure 3.2: Original SLCC with overall geometry and farfield boundary conditions.

Inlet Boundary Conditions

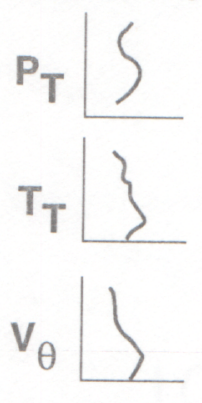

Mass Flow $=$ Constant
Exit Boundary Condition

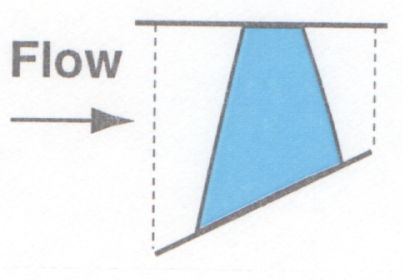

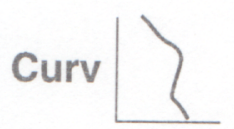

Figure 3.3: Modifications to SLCC for calculating turbomachinery source terms. 

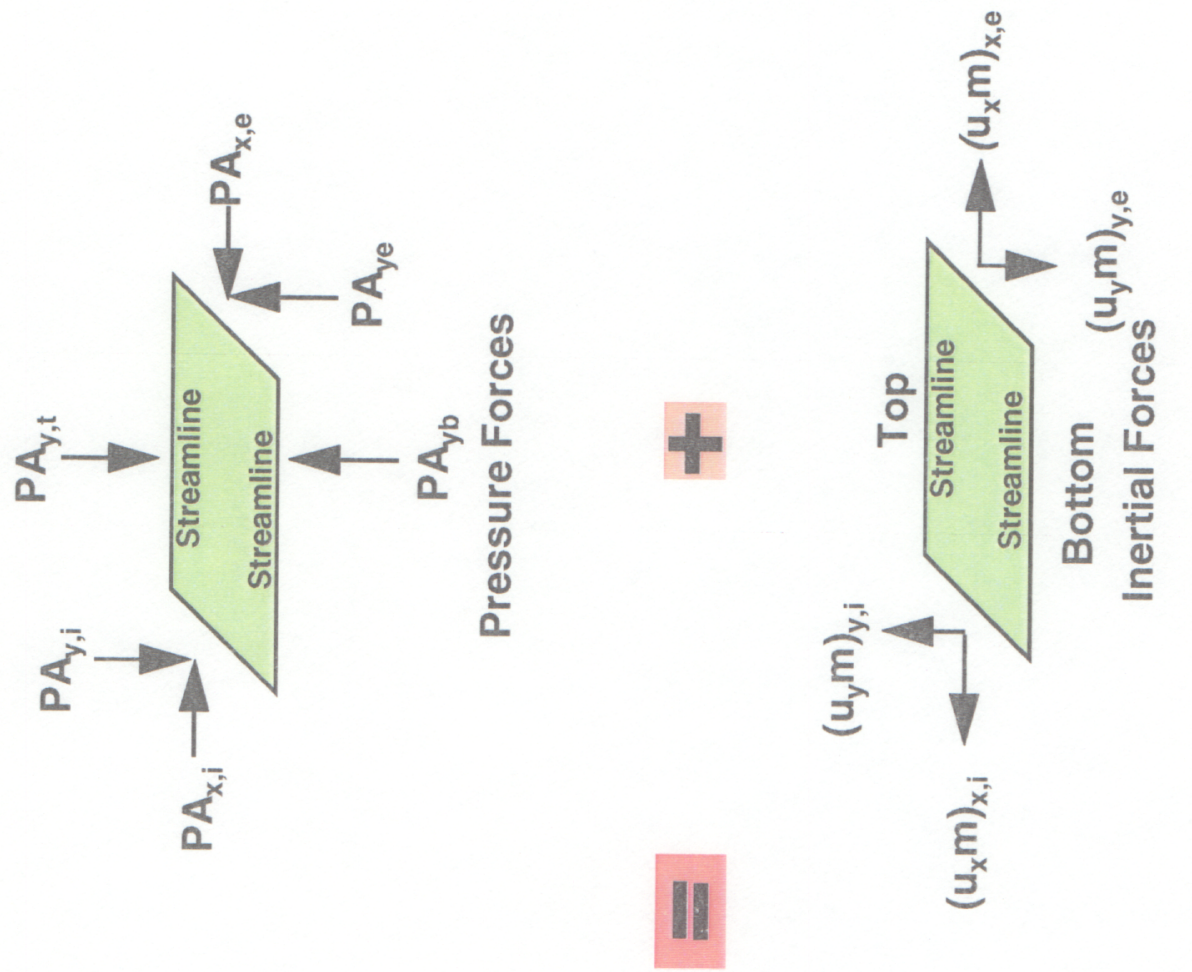

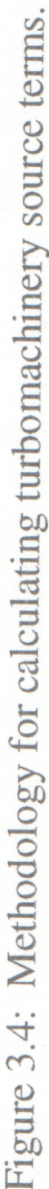




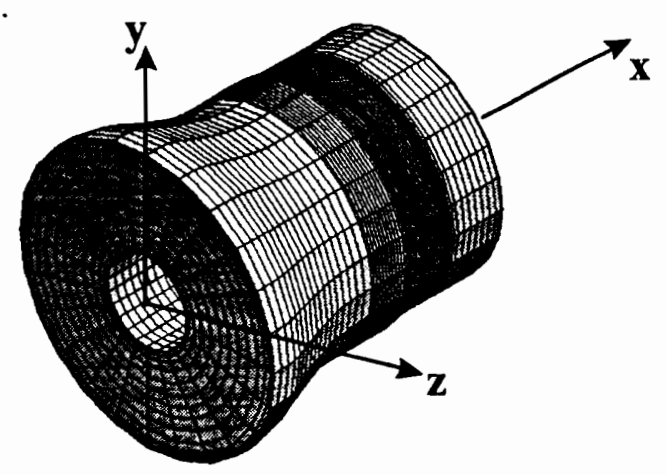

a. Three-dimensional view of grid

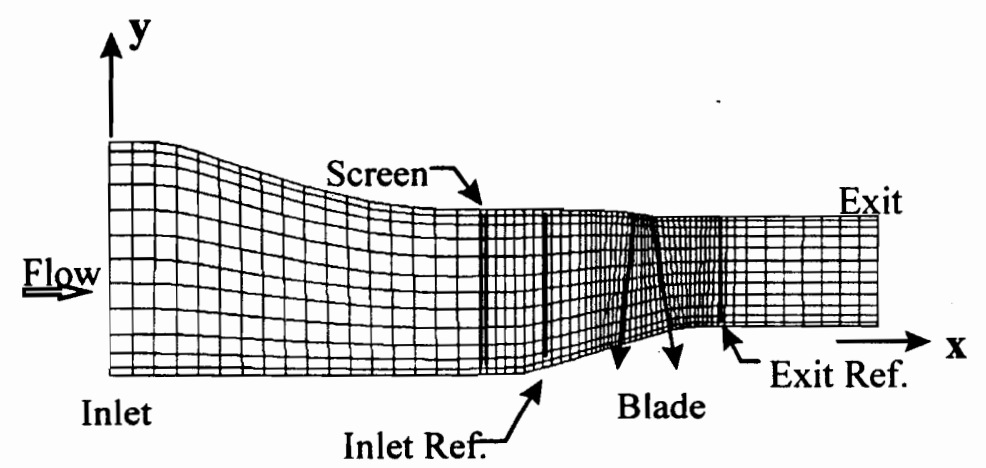

b. Circumferential slice

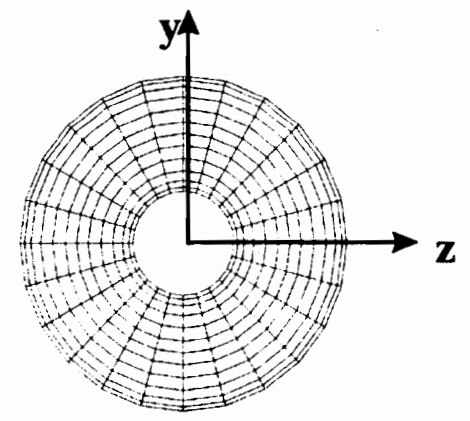

c. Axial slice

Figure 3.5: Cylindrical grid used by TEACC for turbomachinery with axial grid packed through the blades and equally spaced circumferential segments. 


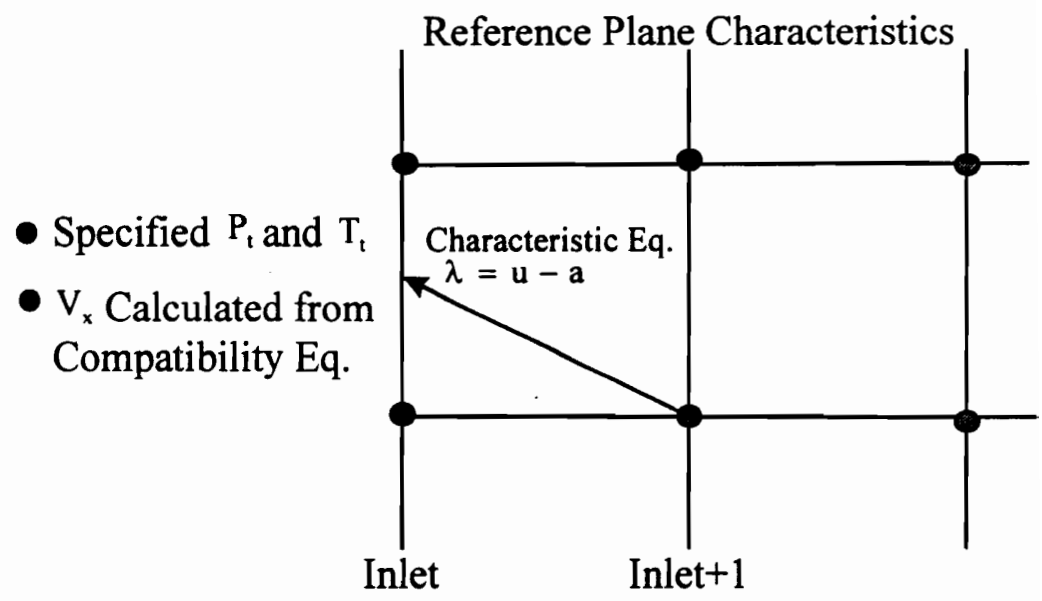

Figure 3.6: Inlet boundary condition.

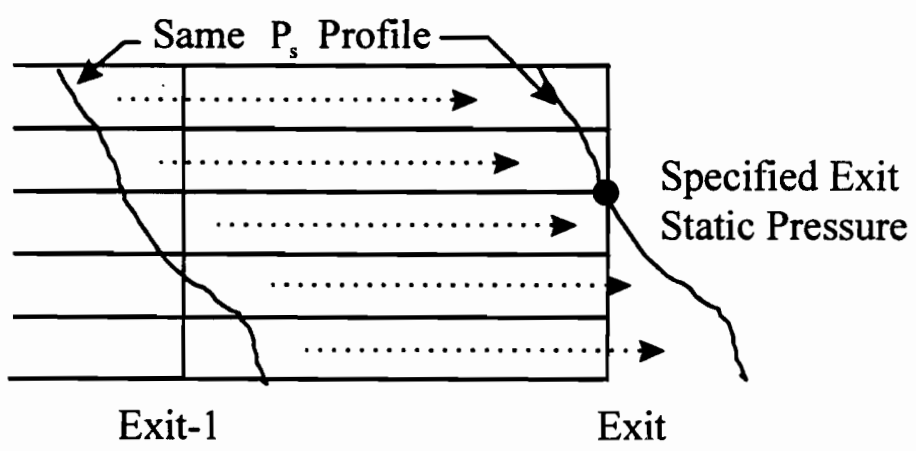

Figure 3.7: Exit boundary condition.

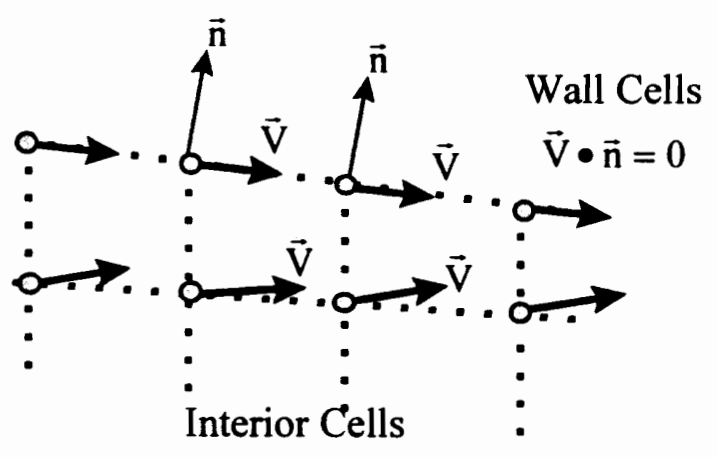

Figure 3.8: Wall boundary condition. 


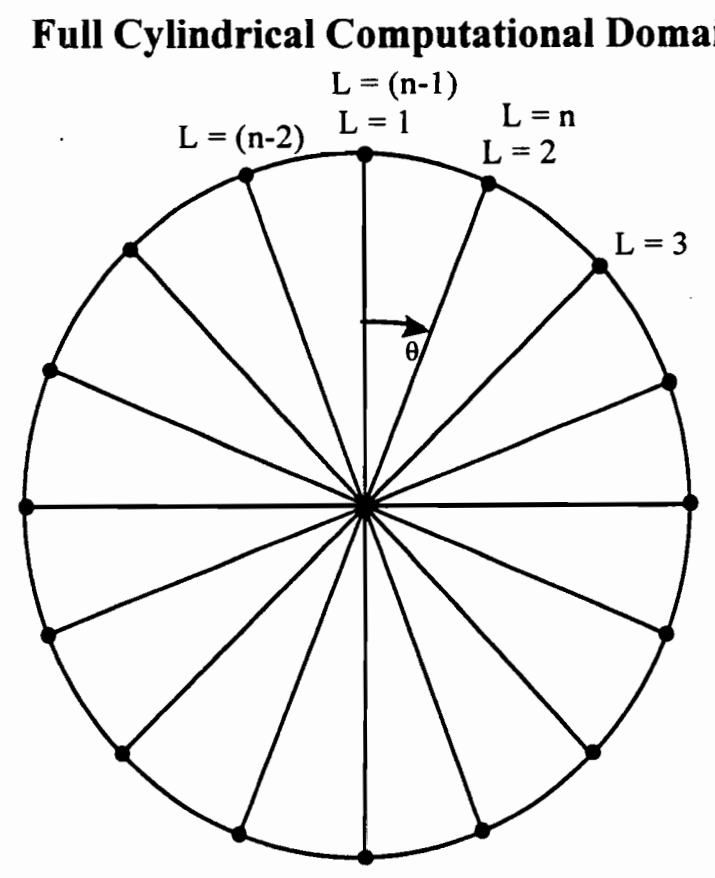

Figure 3.9: Rotationally periodic (wrap-around) boundary condition.

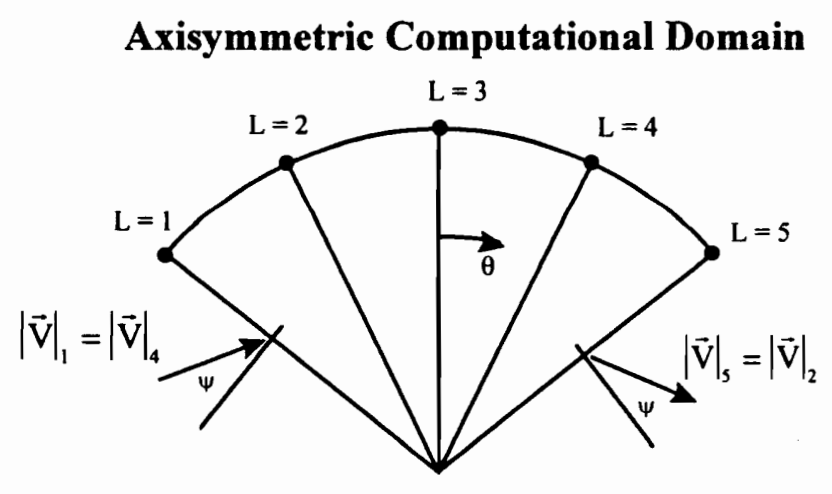

Figure 3.10: Rotationally cyclic boundary condition. 


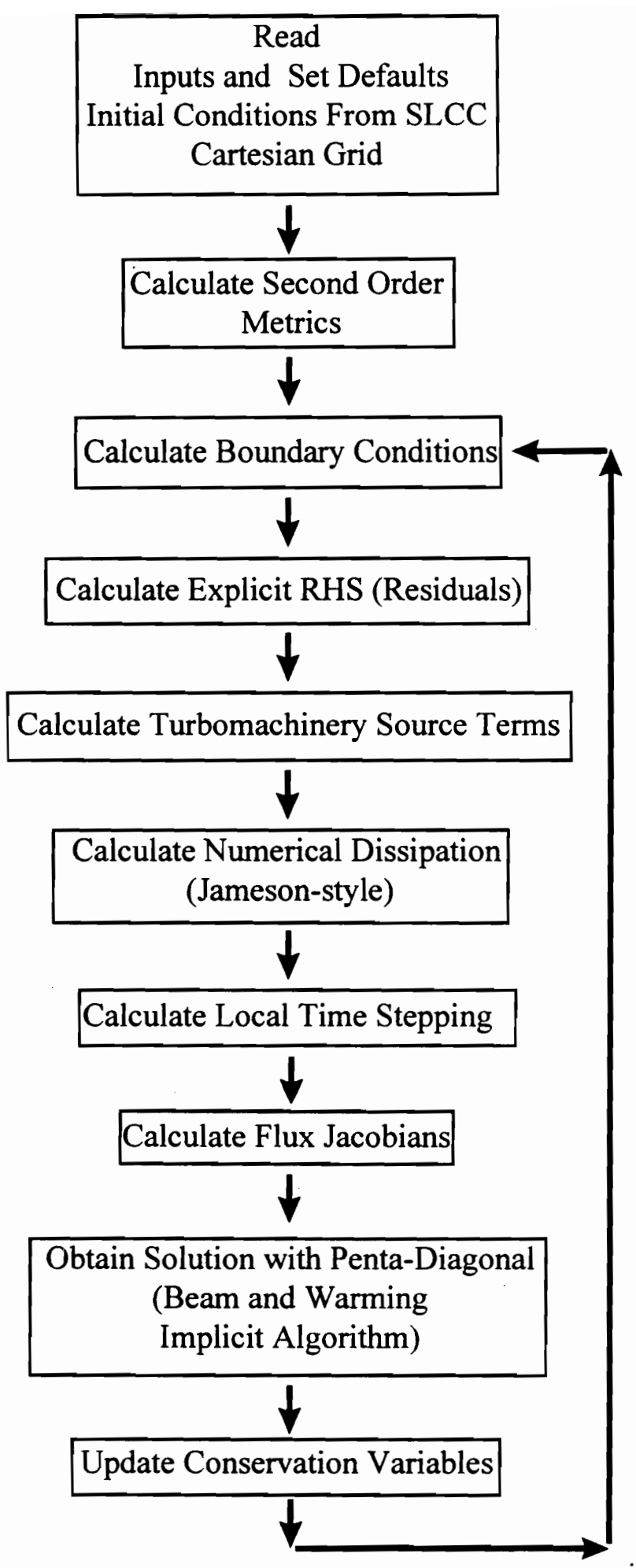

Figure 3.11: TEACC solution procedure flow chart. 

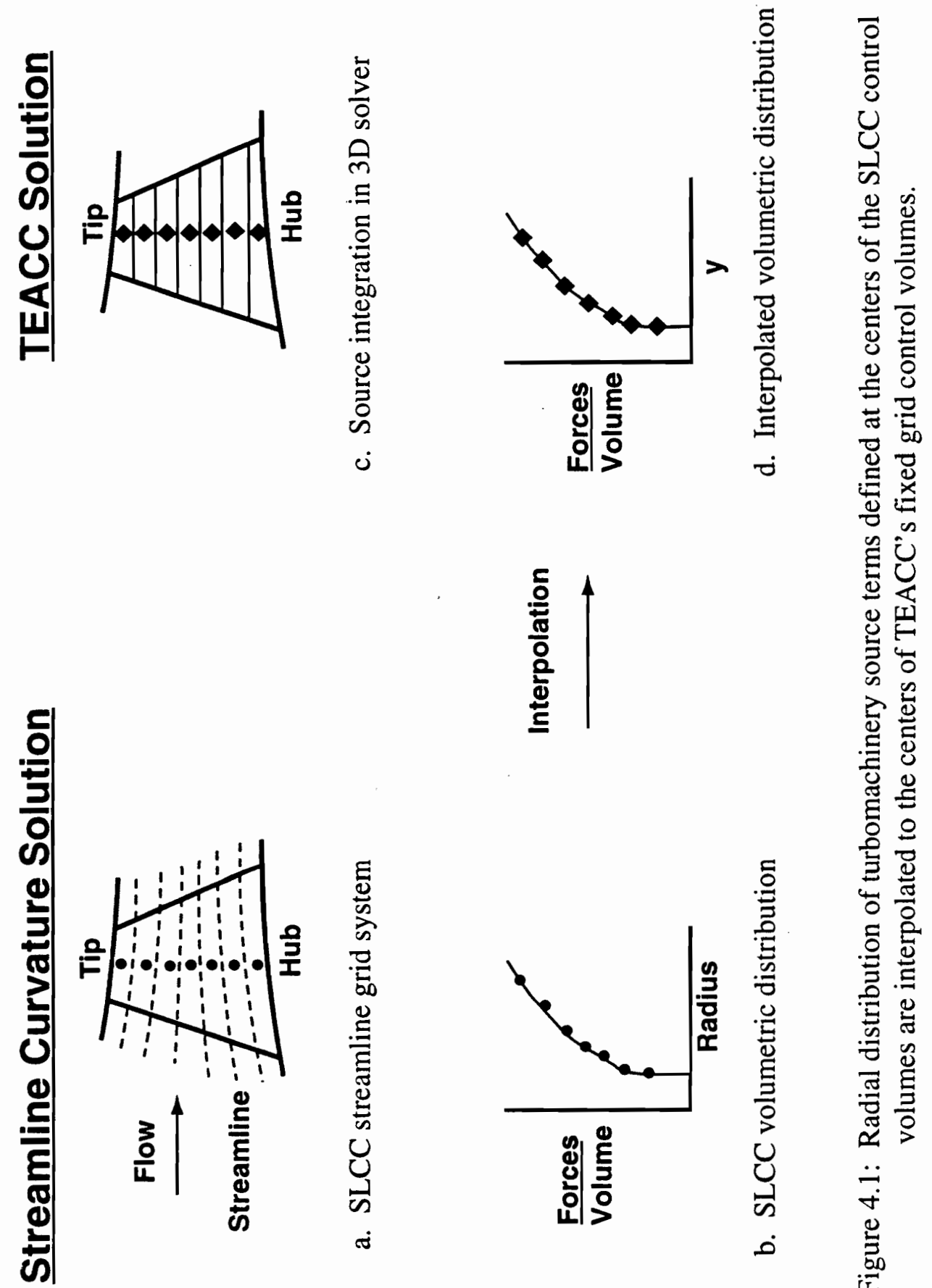

है

\& 띤

응

की

离

胥

응

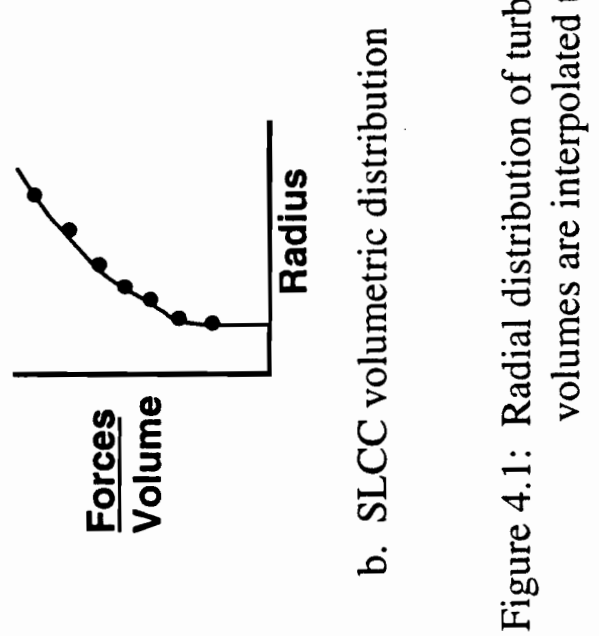



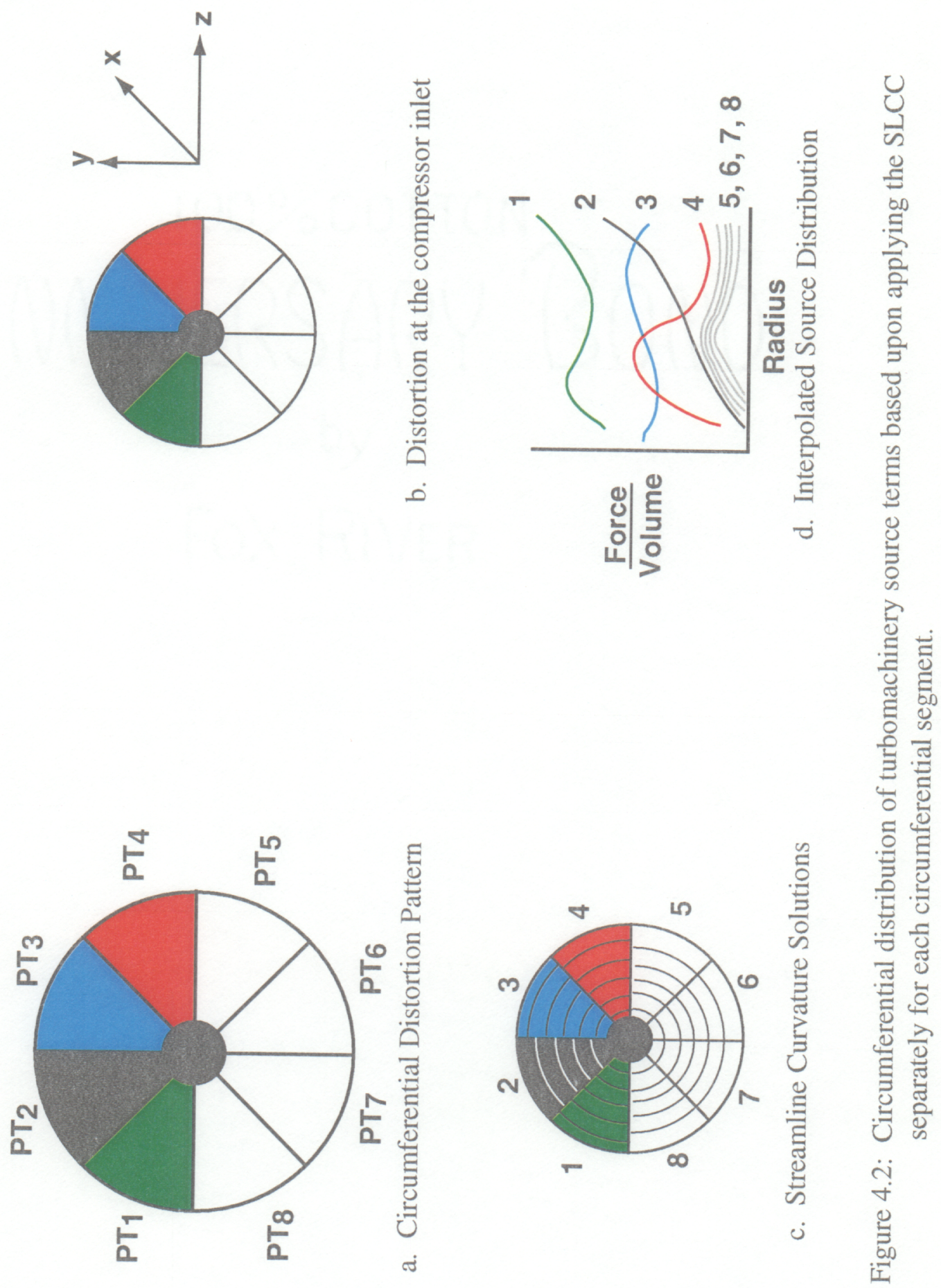


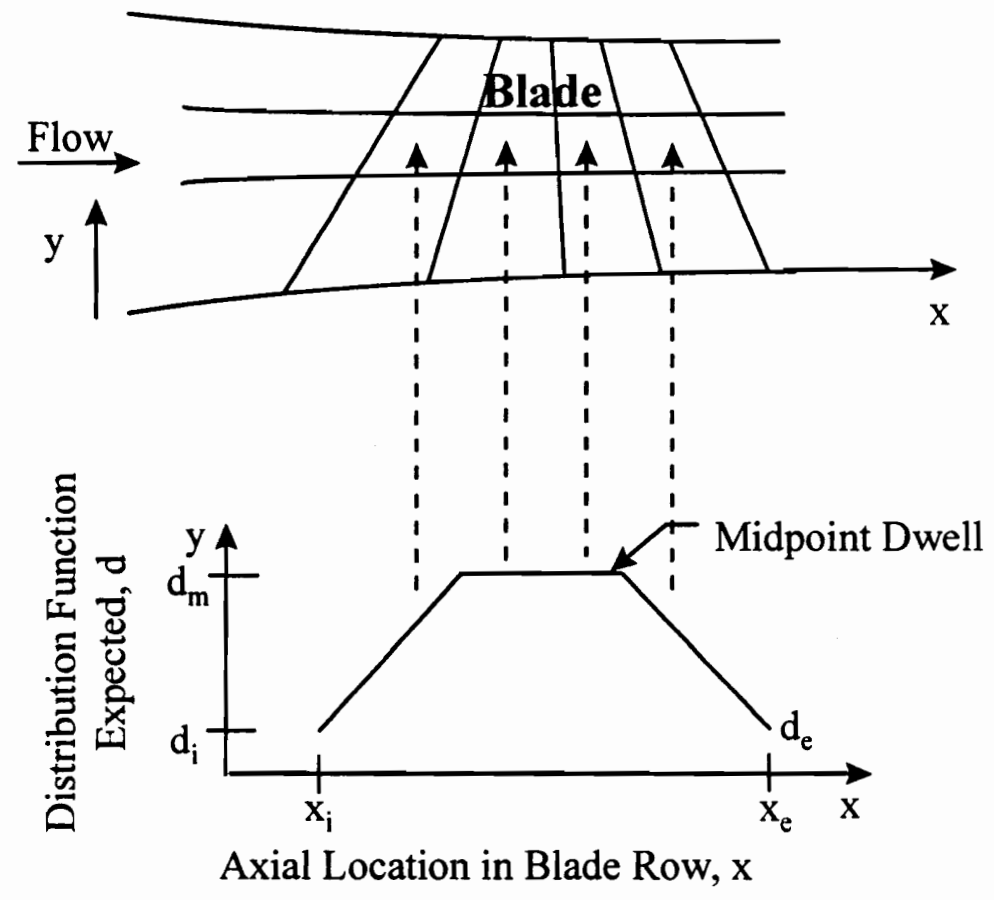

a. TEACC grid with ramped distribution function through the bladed region

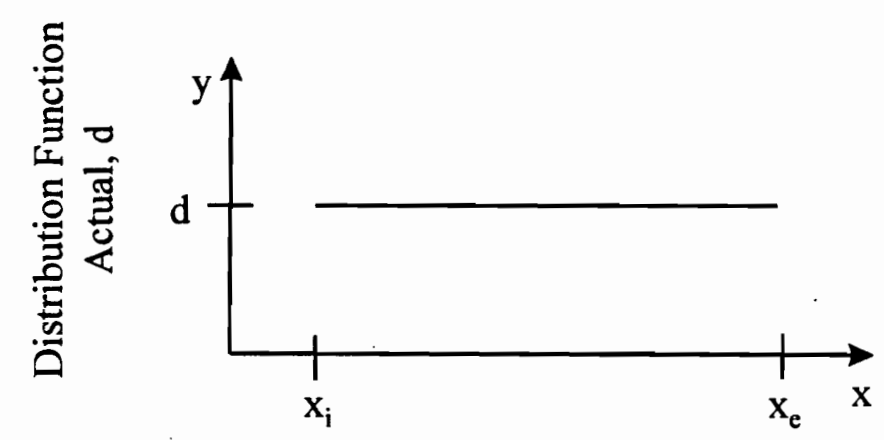

Axial Location in Blade Row, $x$

b. Best distribution function is uniform

Figure 4.3: Axial distribution of turbomachinery source terms. 


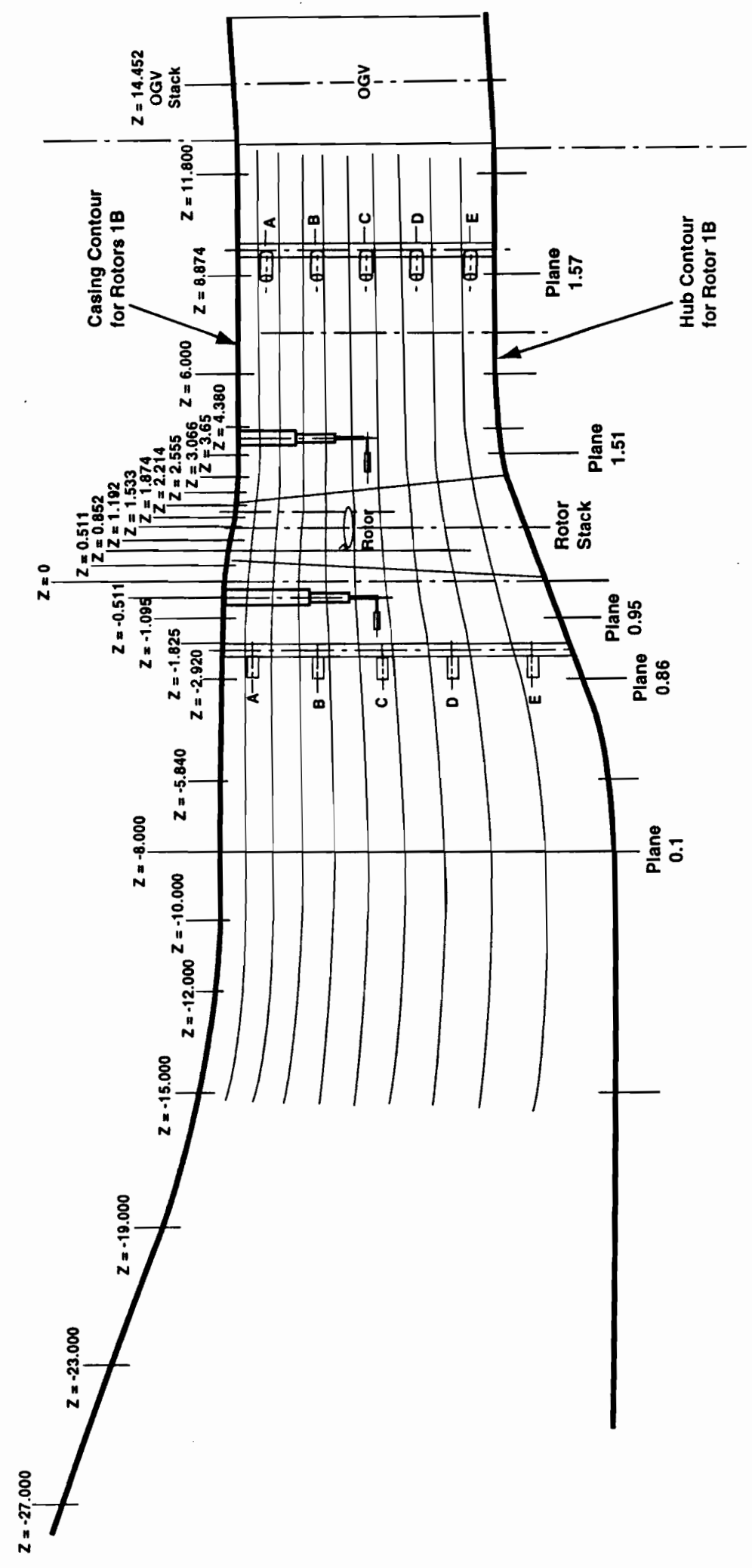

0
0
0
0
0
0
0
0
0
0
0
0
0
0
0
0
0
0
0
0
0

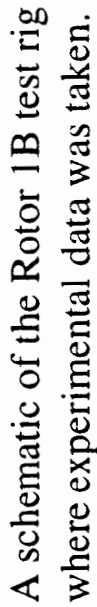

的 


\section{Distortion Screen}

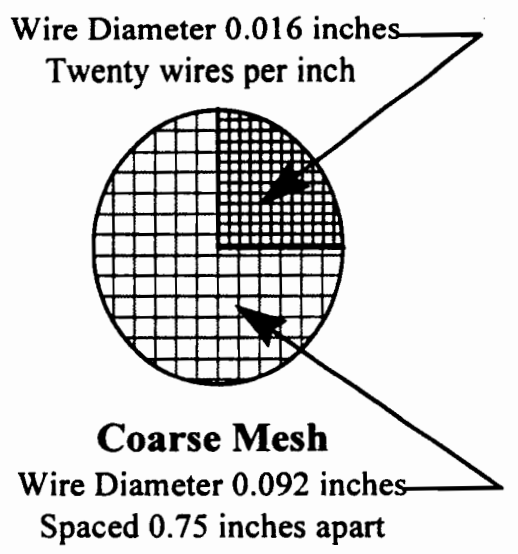

Figure 5.2: 90-degree, one-per-revolution distortion screen. 


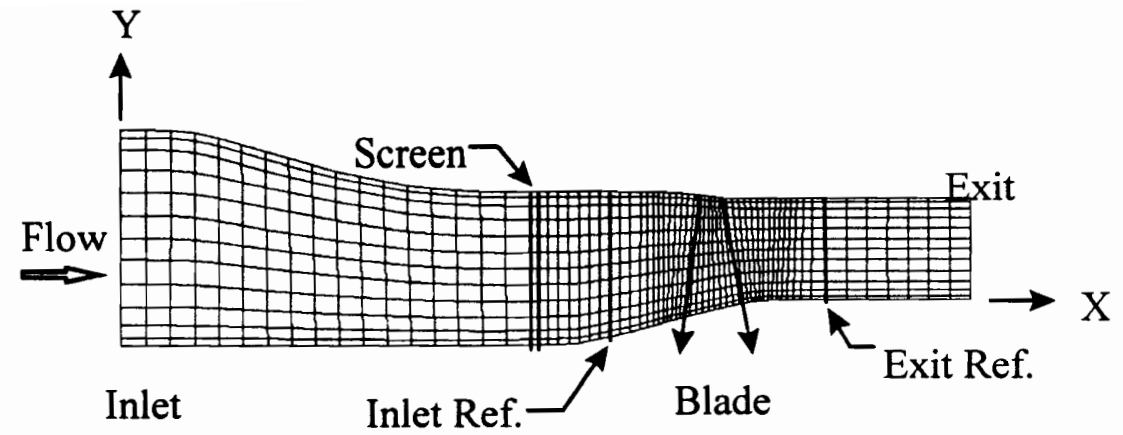

Figure 6.1: Axial-radial view of the three-dimensional base grid modeling Rotor $1 \mathrm{~B}$ and the test facility, with grid nodes $(69 \times 13 \times 26)$.

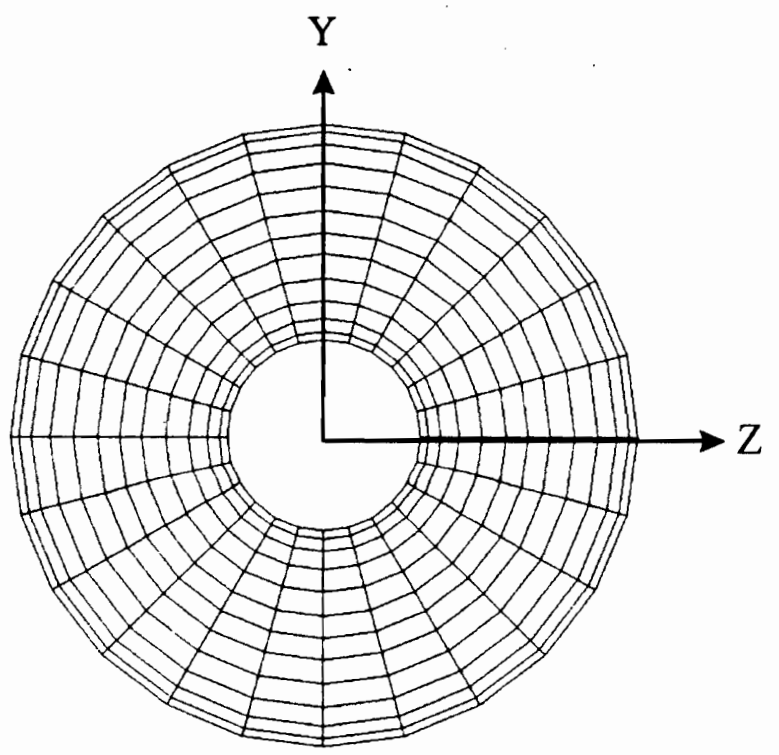

Figure 6.2: Radial-circumferential view of the three-dimensional base grid modeling Rotor 1B and the test facility, with grid nodes $(69 \times 13 \times 26)$. 


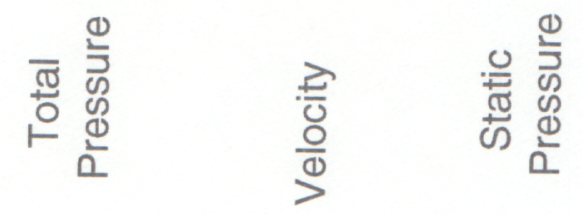

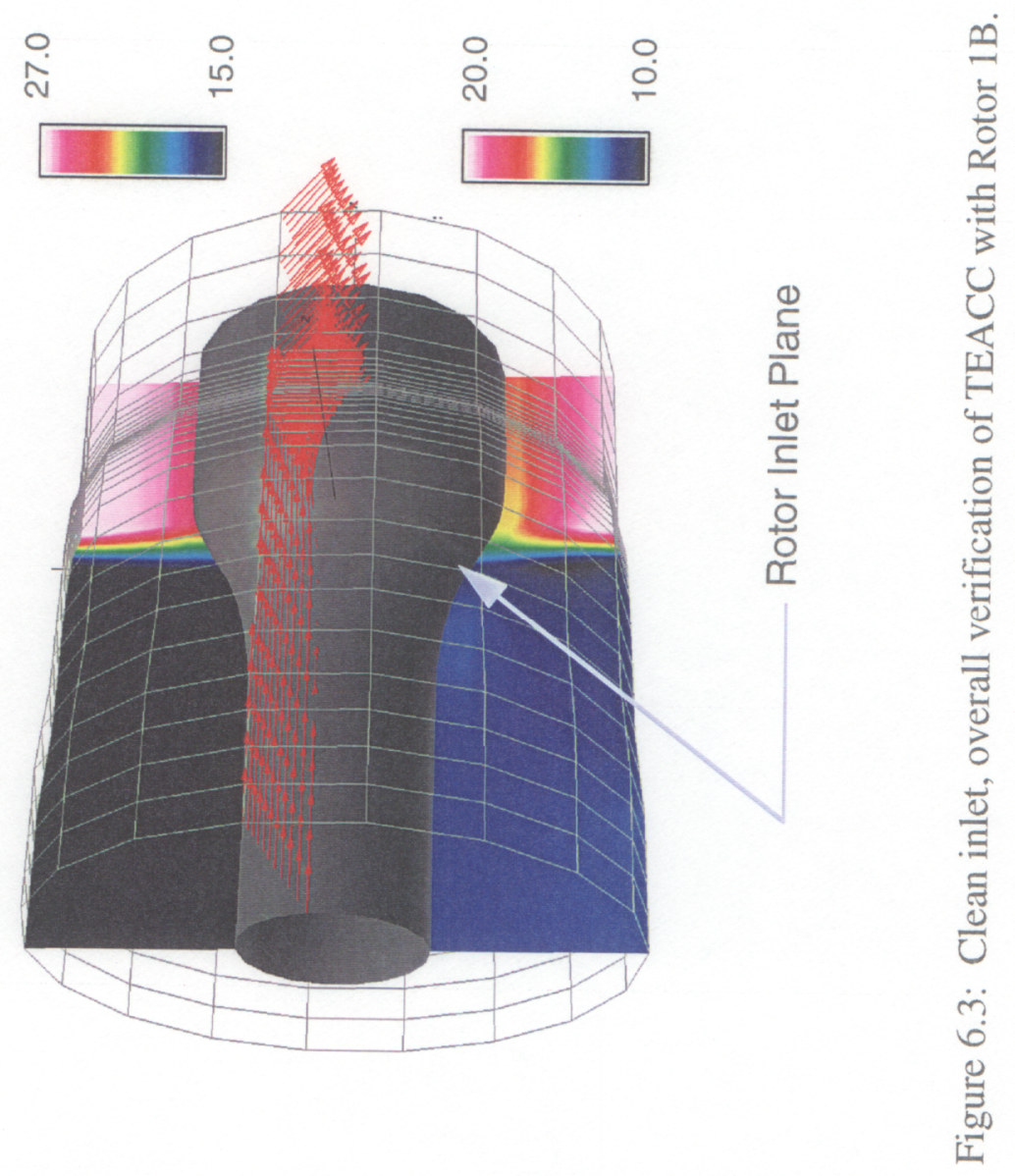

은 

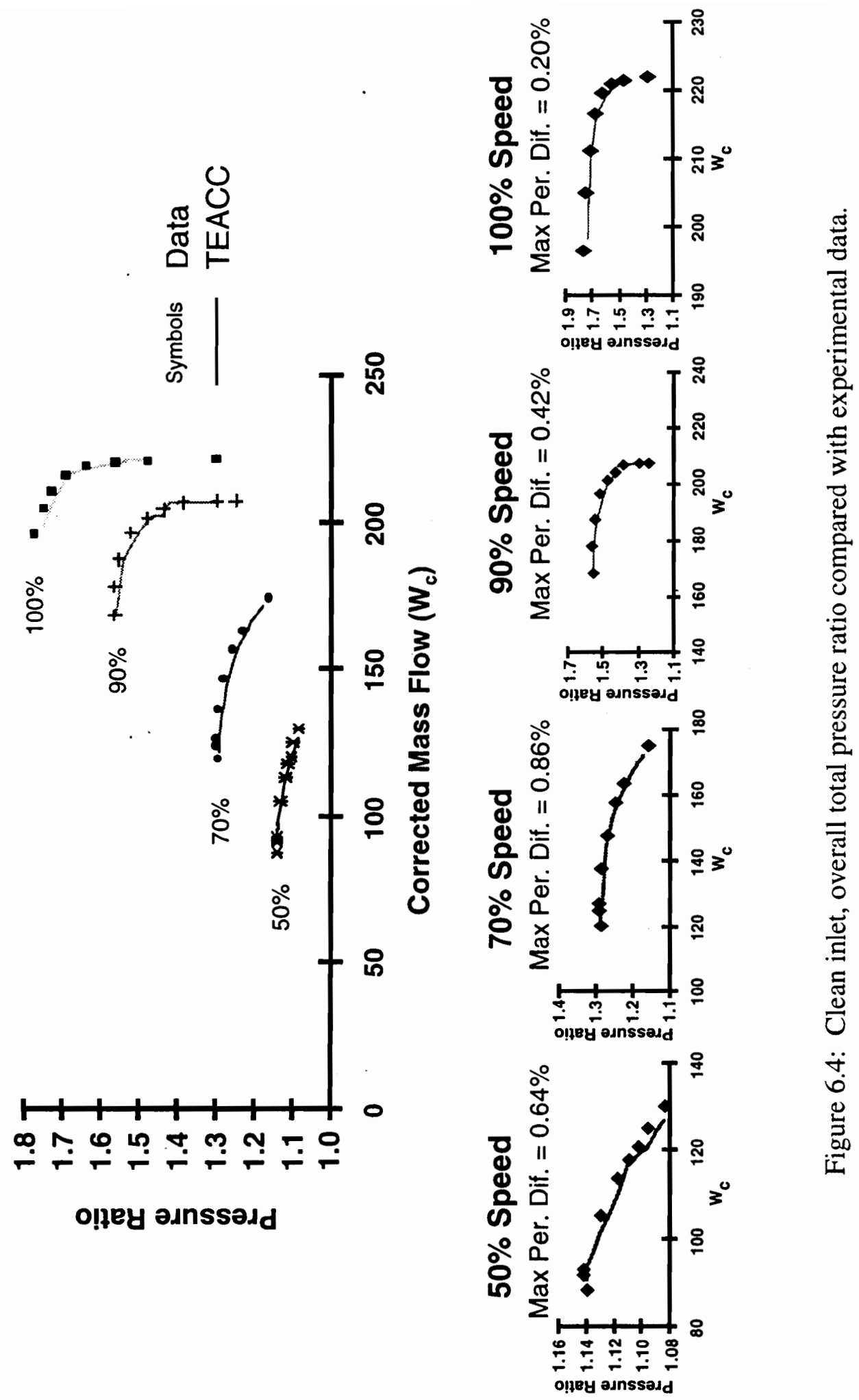

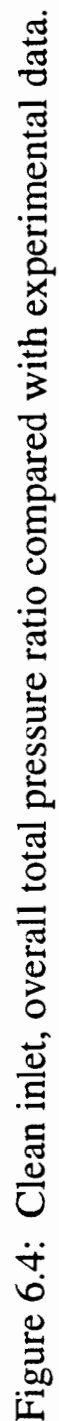



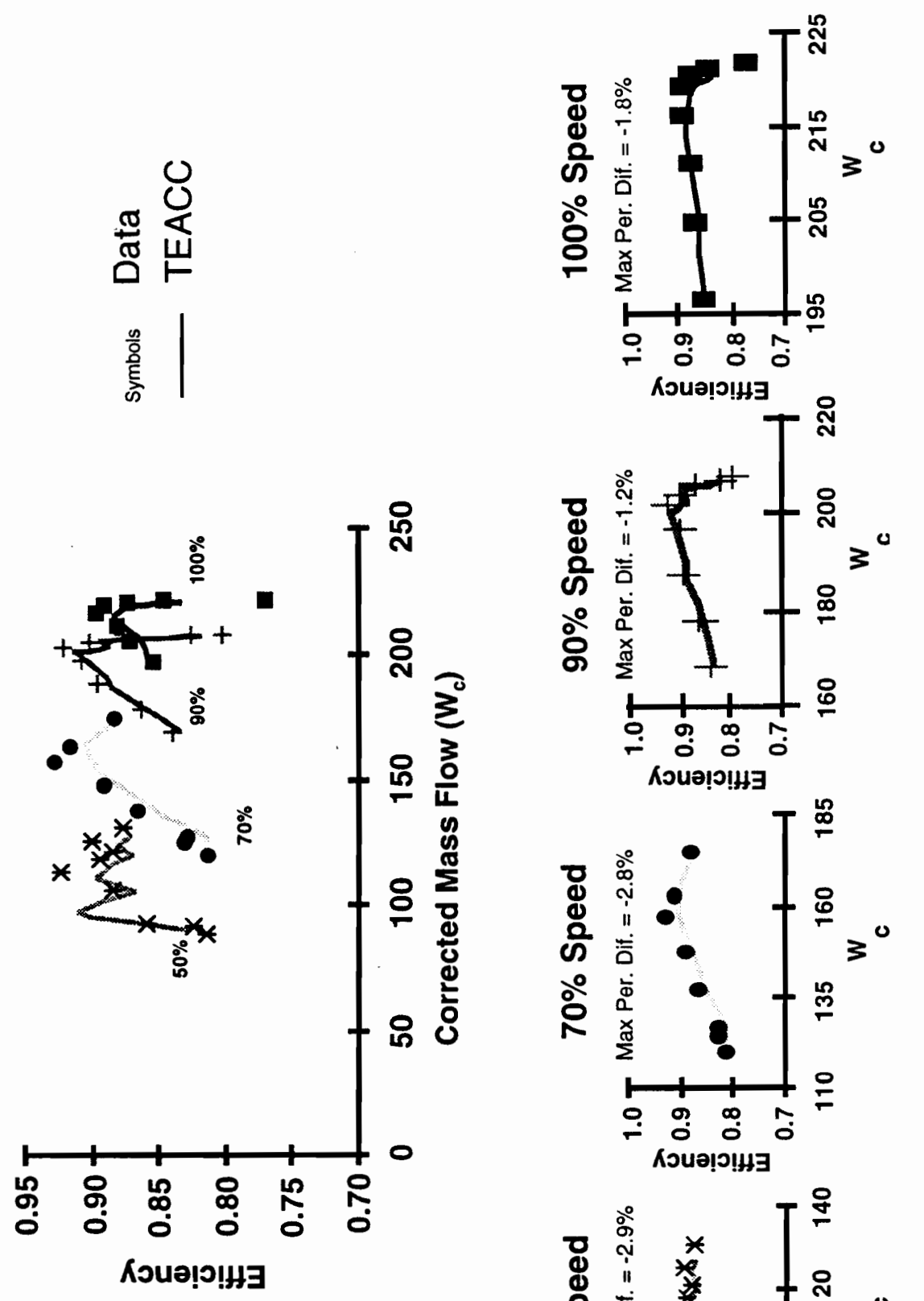

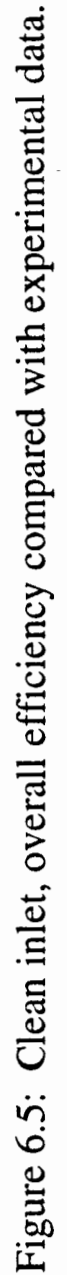

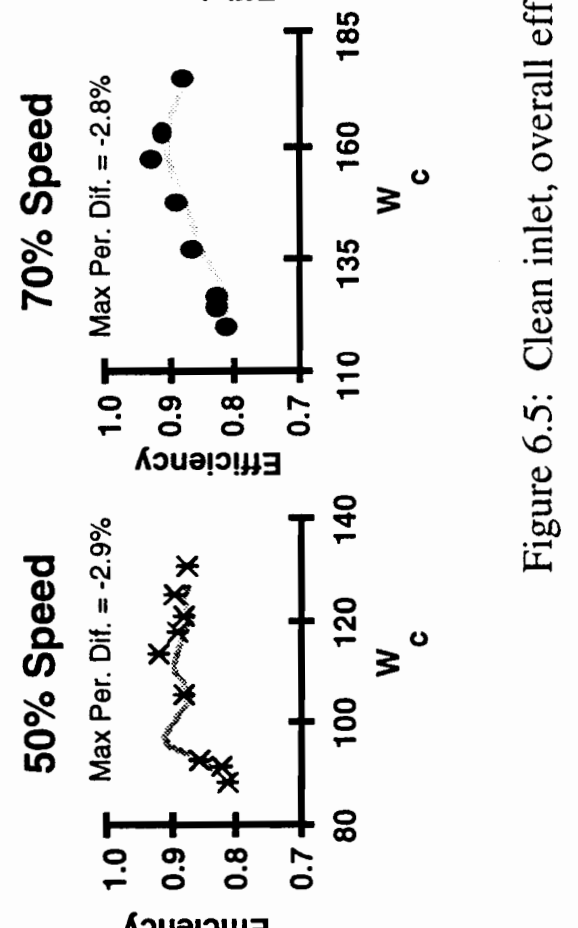




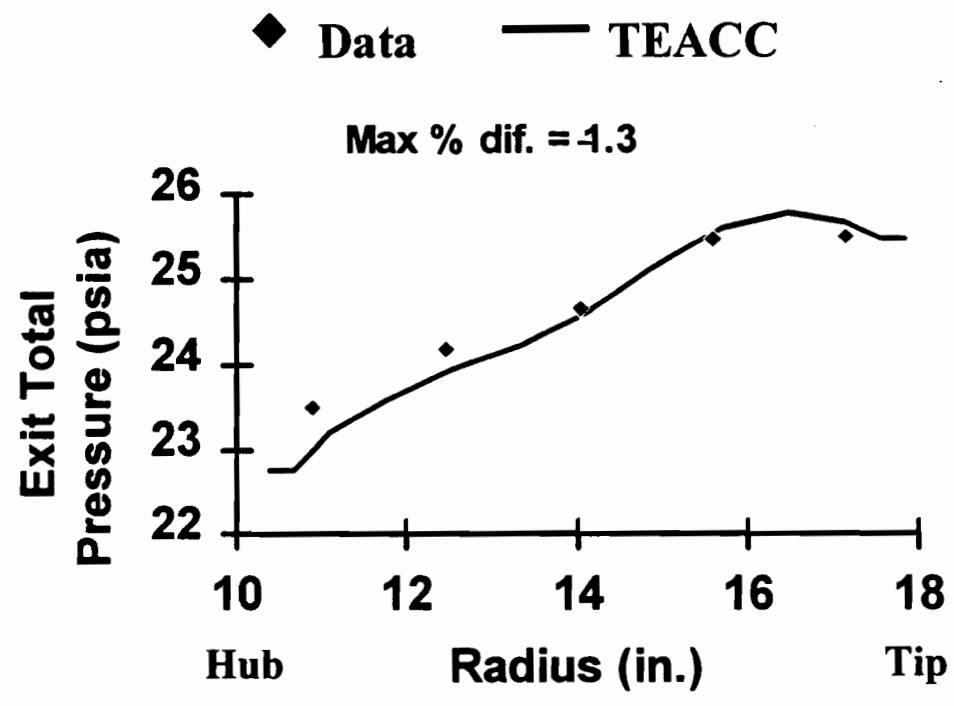

Figure 6.6: Clean inlet, exit total pressure on the 100\% corrected speed line near the design throttle line.

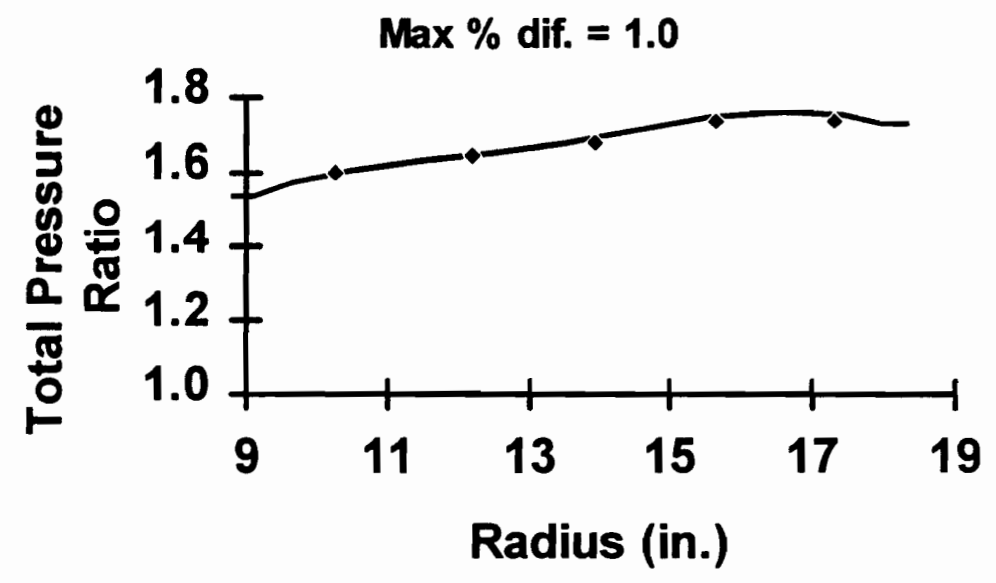

Figure 6.7: Clean inlet, total pressure ratio on the $100 \%$ corrected speed line near the design throttle line. 


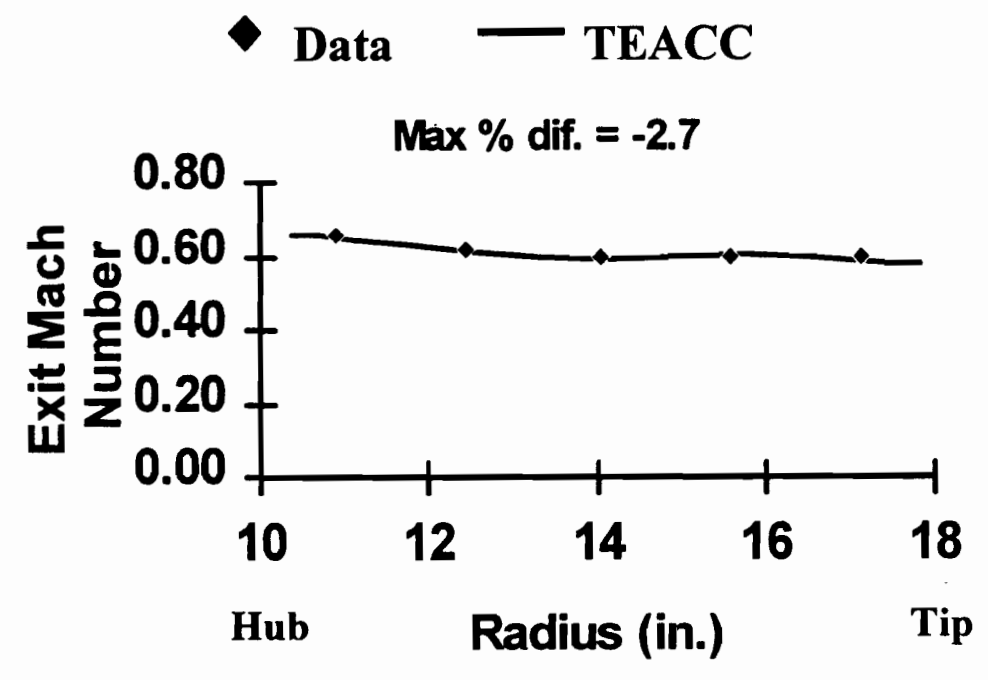

Figure 6.8: Clean inlet, exit Mach number on the 100\% corrected speed line near the design throttle line.

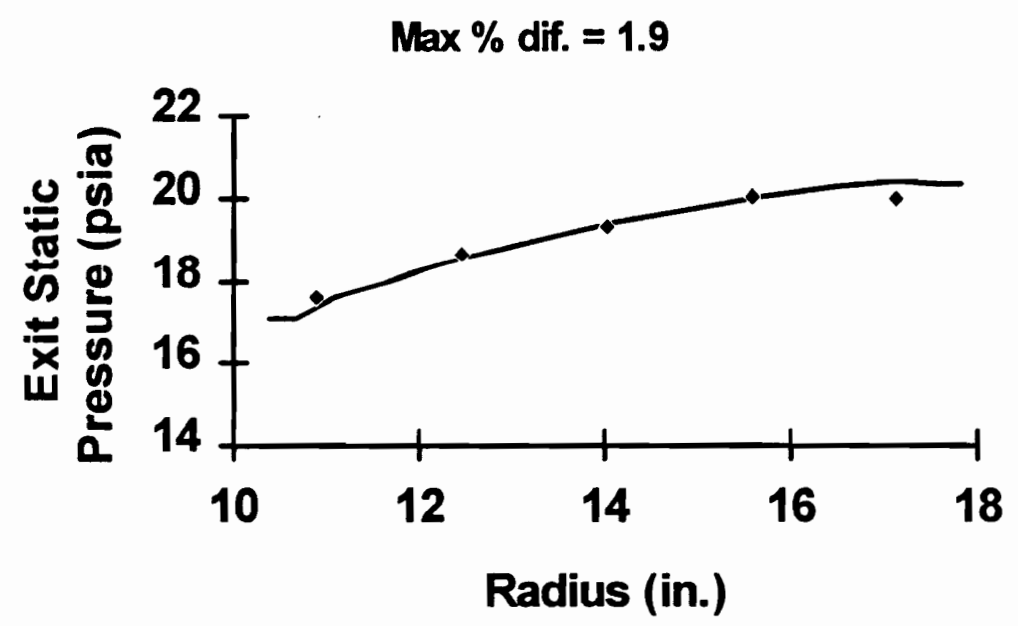

Figure 6.9: Clean inlet, exit static pressure on the $100 \%$ corrected speed line near the design throttle line. 
$\checkmark$ Data TEACC

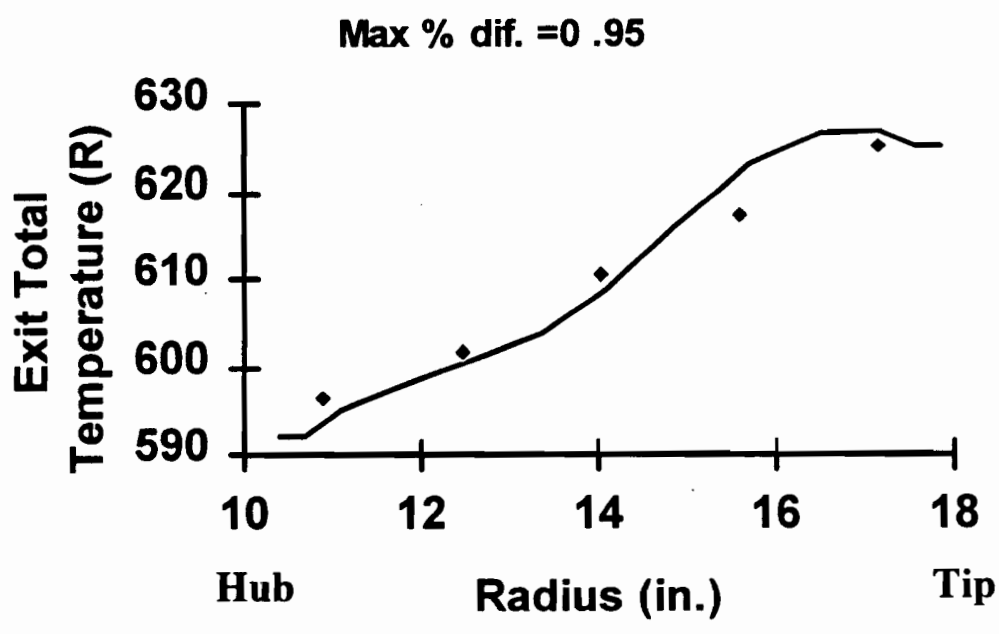

Figure 6.10: Clean inlet, exit total temperature on the $100 \%$ corrected speed line near the design throttle line.

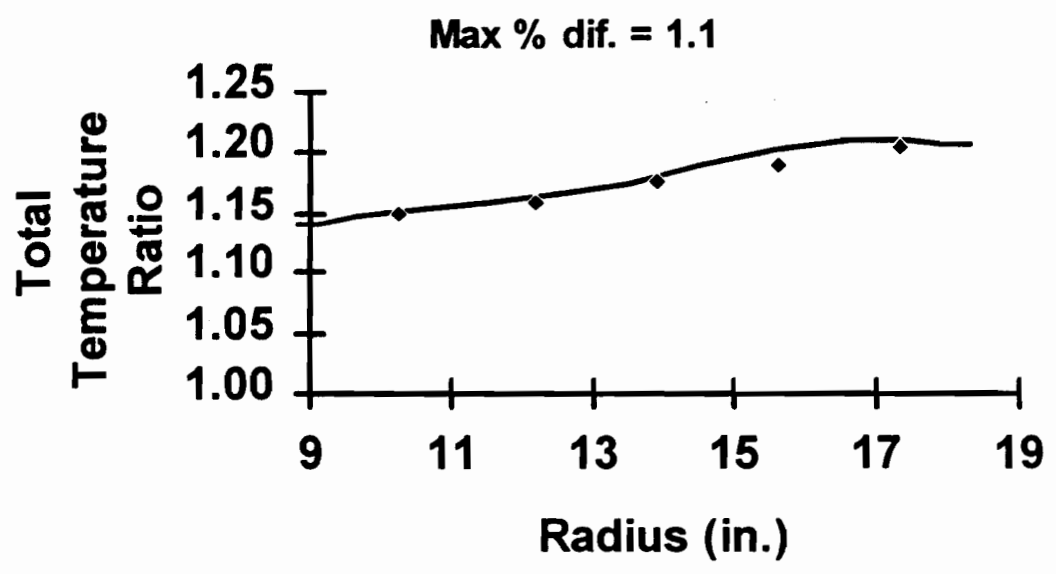

Figure 6.11: Clean inlet, total temperature ratio on the $100 \%$ corrected speed line near the design throttle line. 


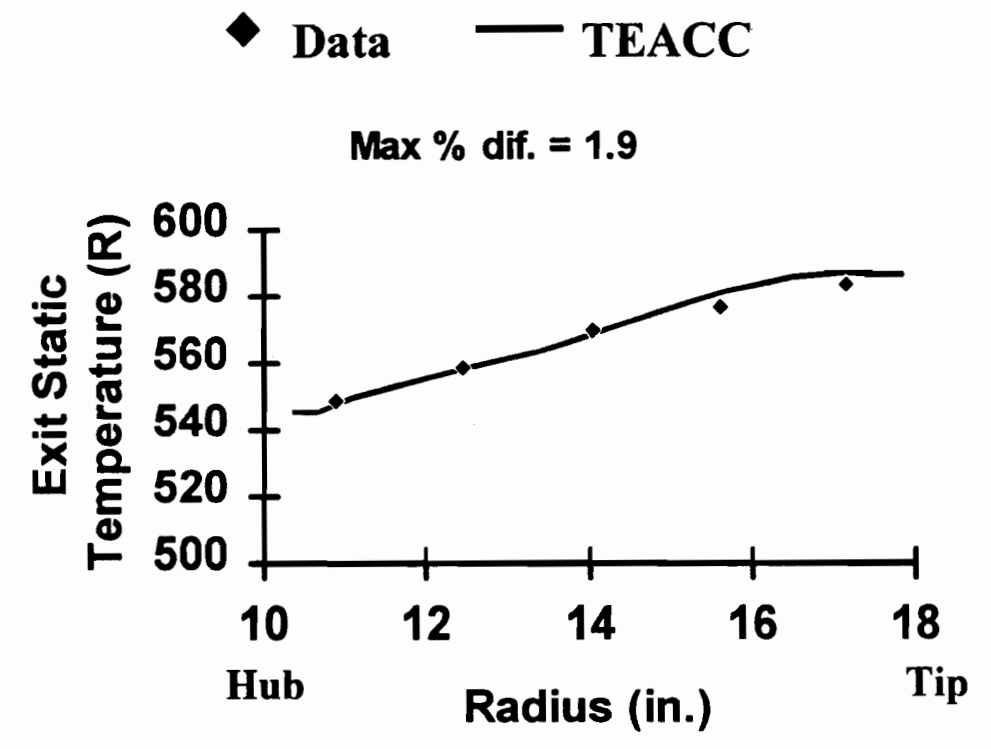

Figure 6.12: Clean inlet, exit static temperature on the $100 \%$ corrected speed line near the design throttle line.

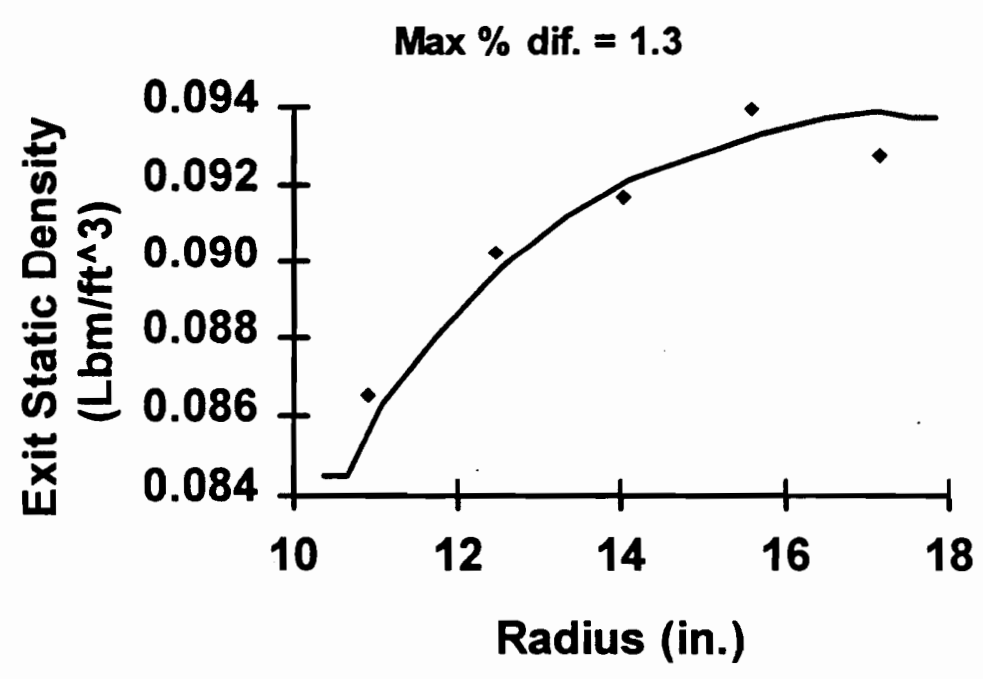

Figure 6.13: Clean inlet, exit static density on the $100 \%$ corrected speed line near the design throttle line. 


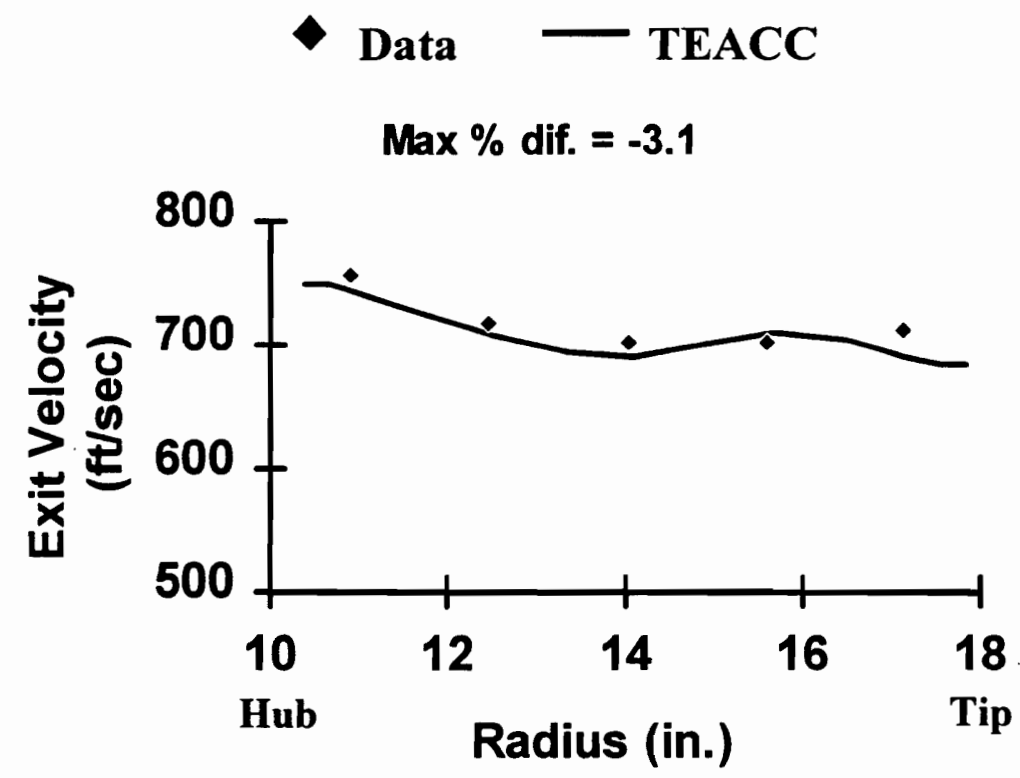

Figure 6.14: Clean inlet, exit absolute velocity on the $100 \%$ corrected speed line near the design throttle line.

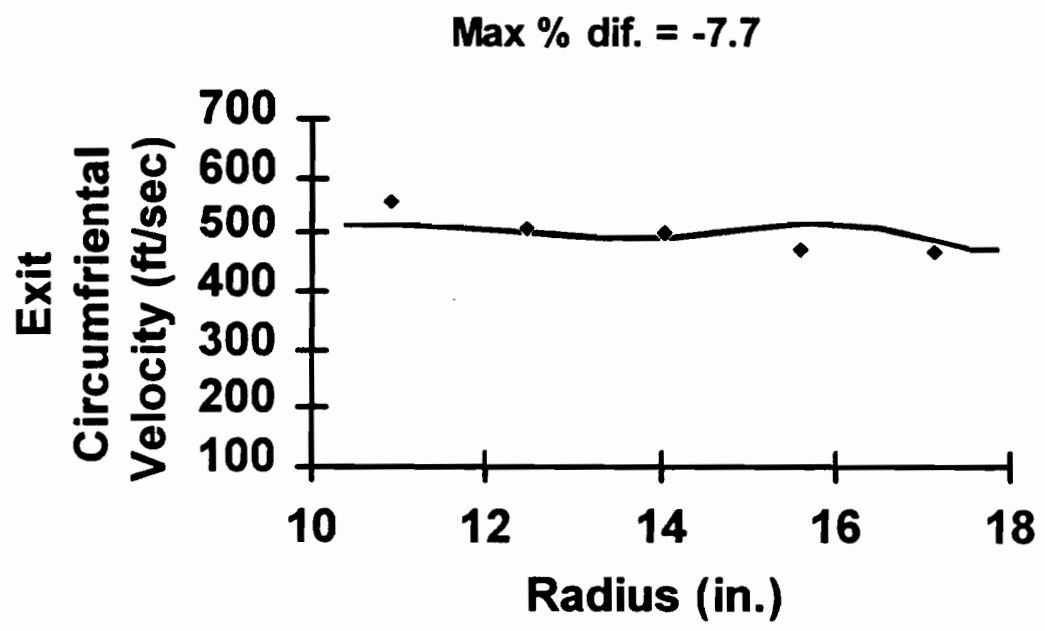

Figure 6.15: Clean inlet, exit circumferential velocity on the $100 \%$ corrected speed line near the design throttle line. 


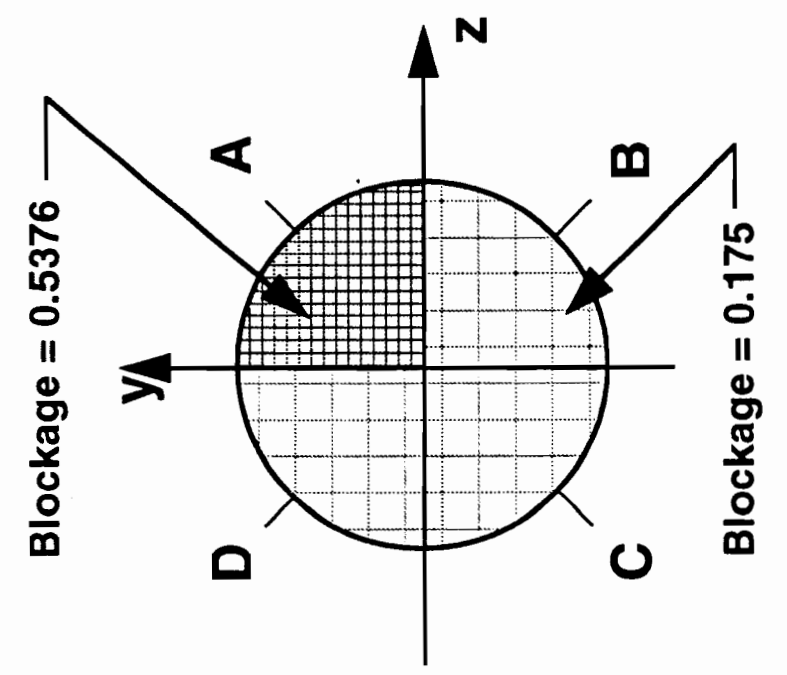

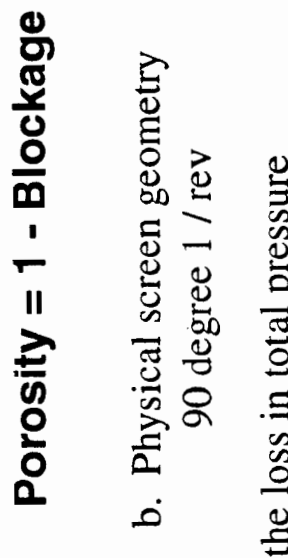

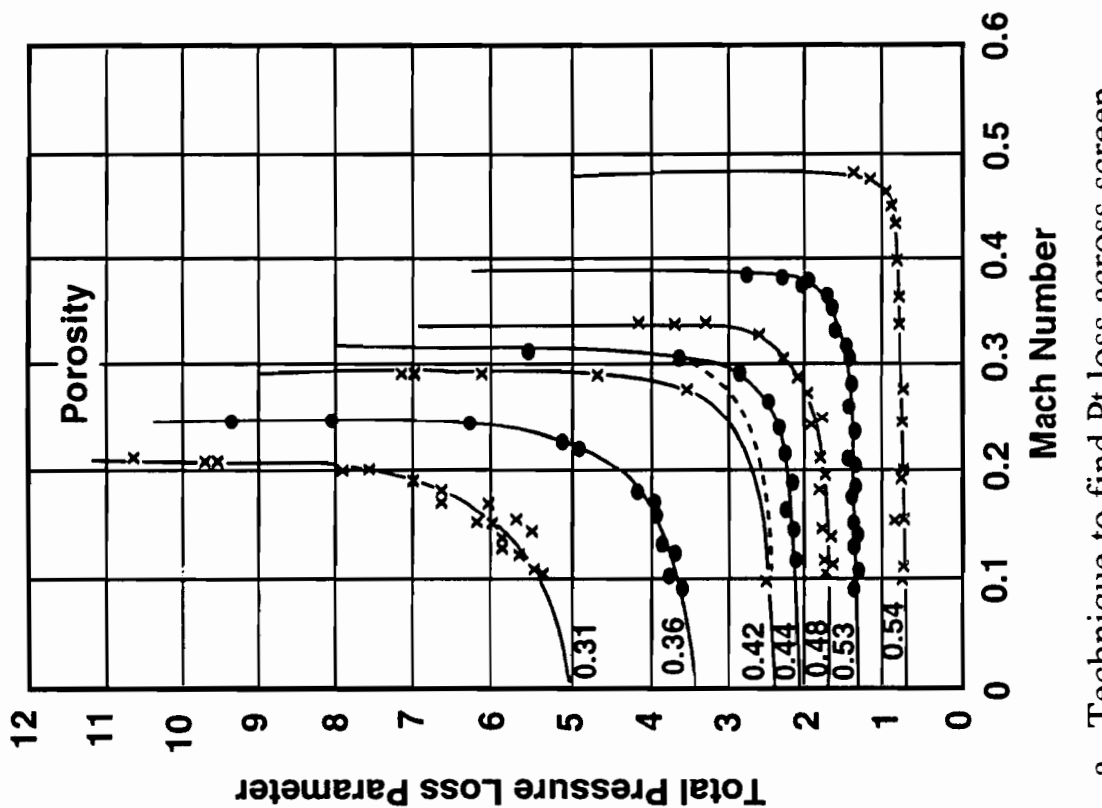

용

证

넌 过

os

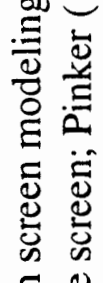

.ํ.

$\stackrel{0}{\circ}$

总 雚

$\ddot{\circ}$

冚 


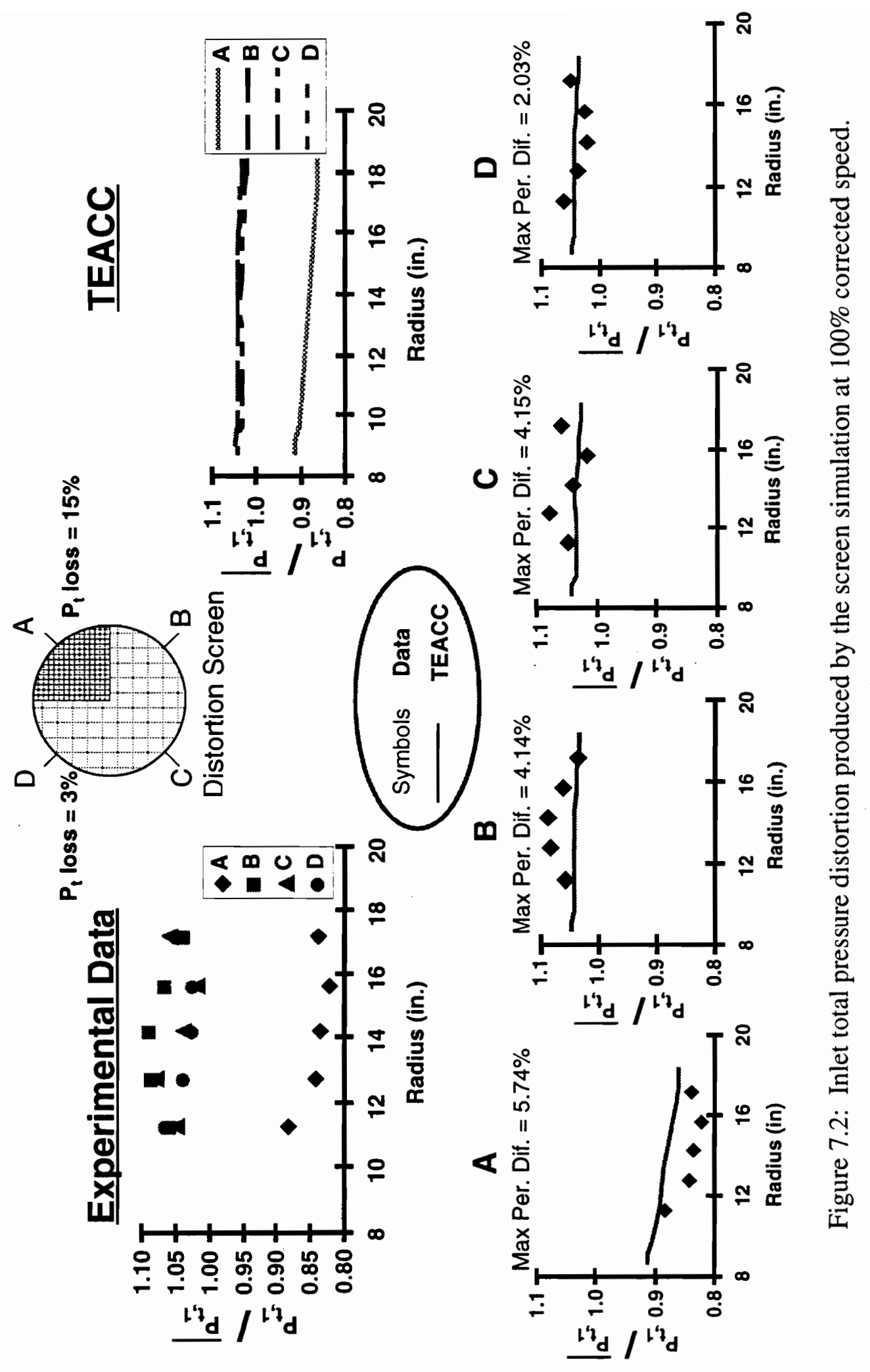




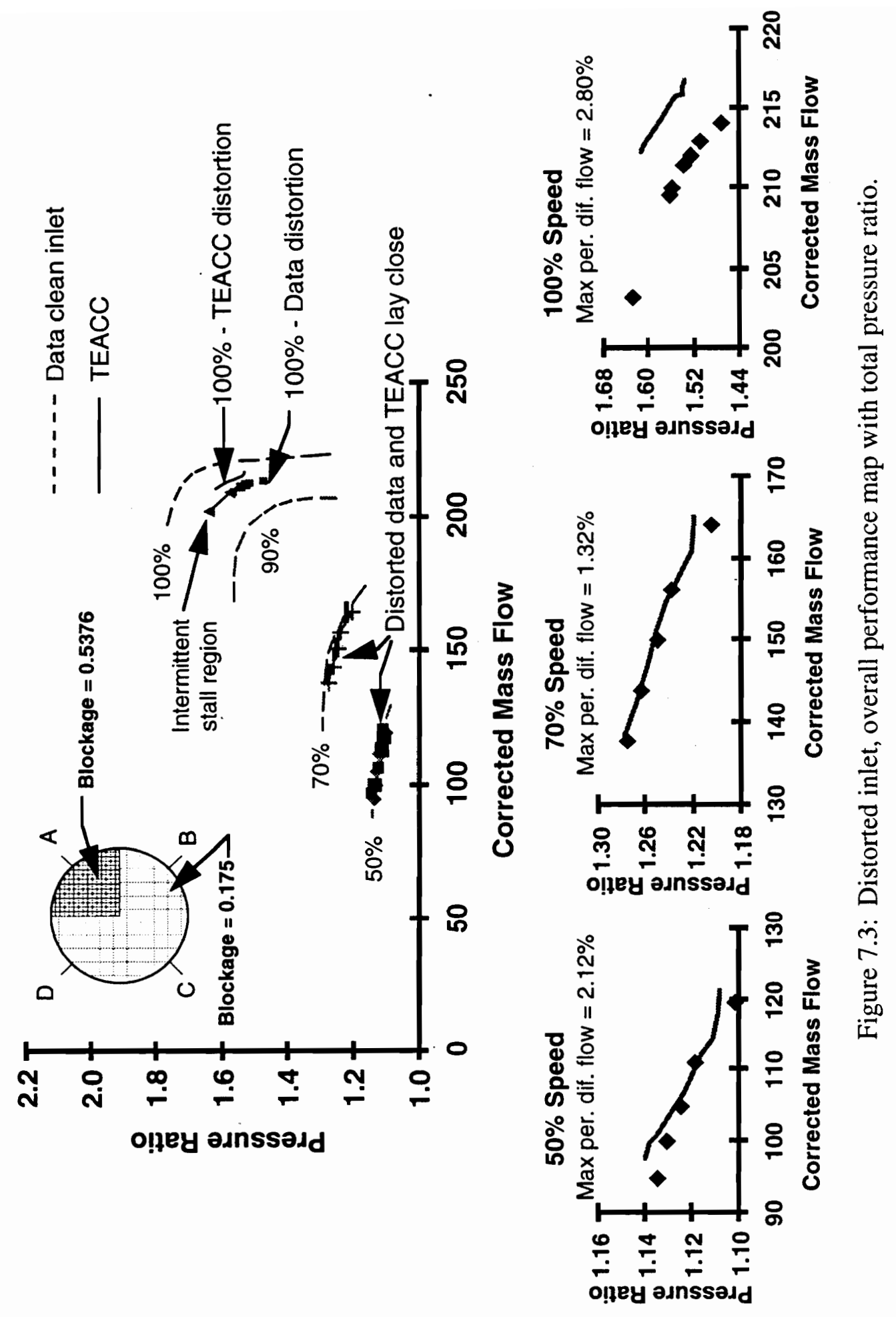




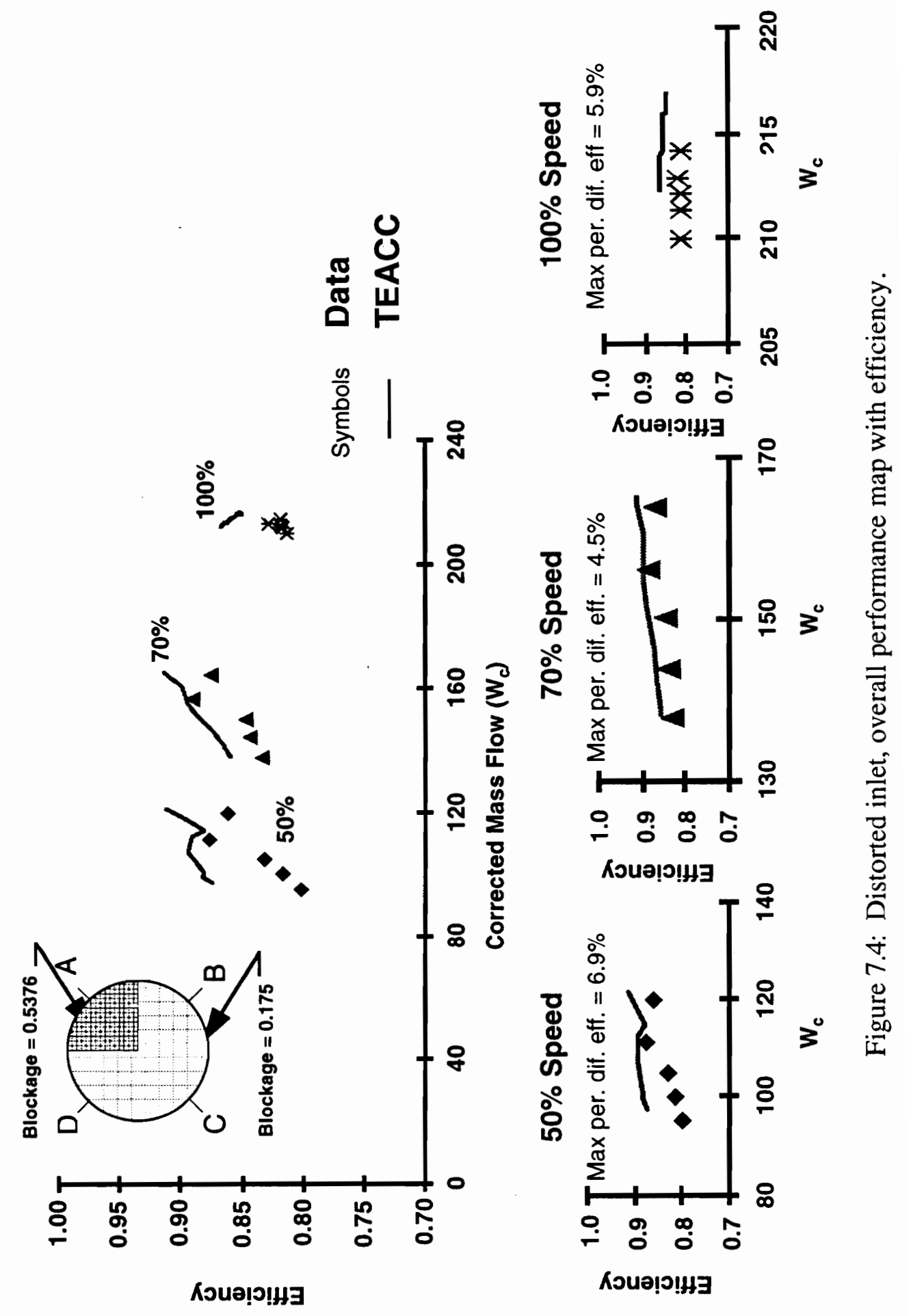



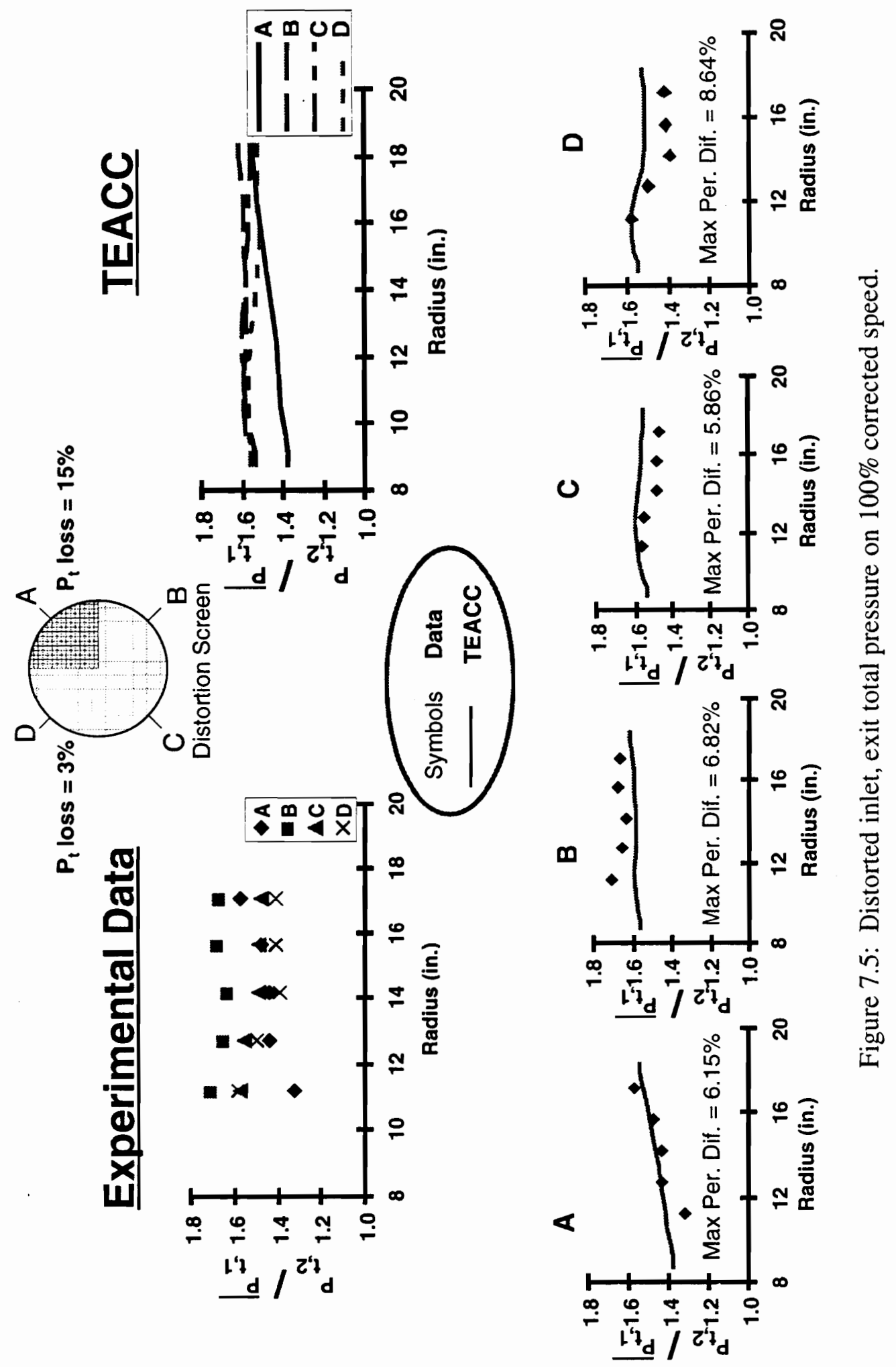

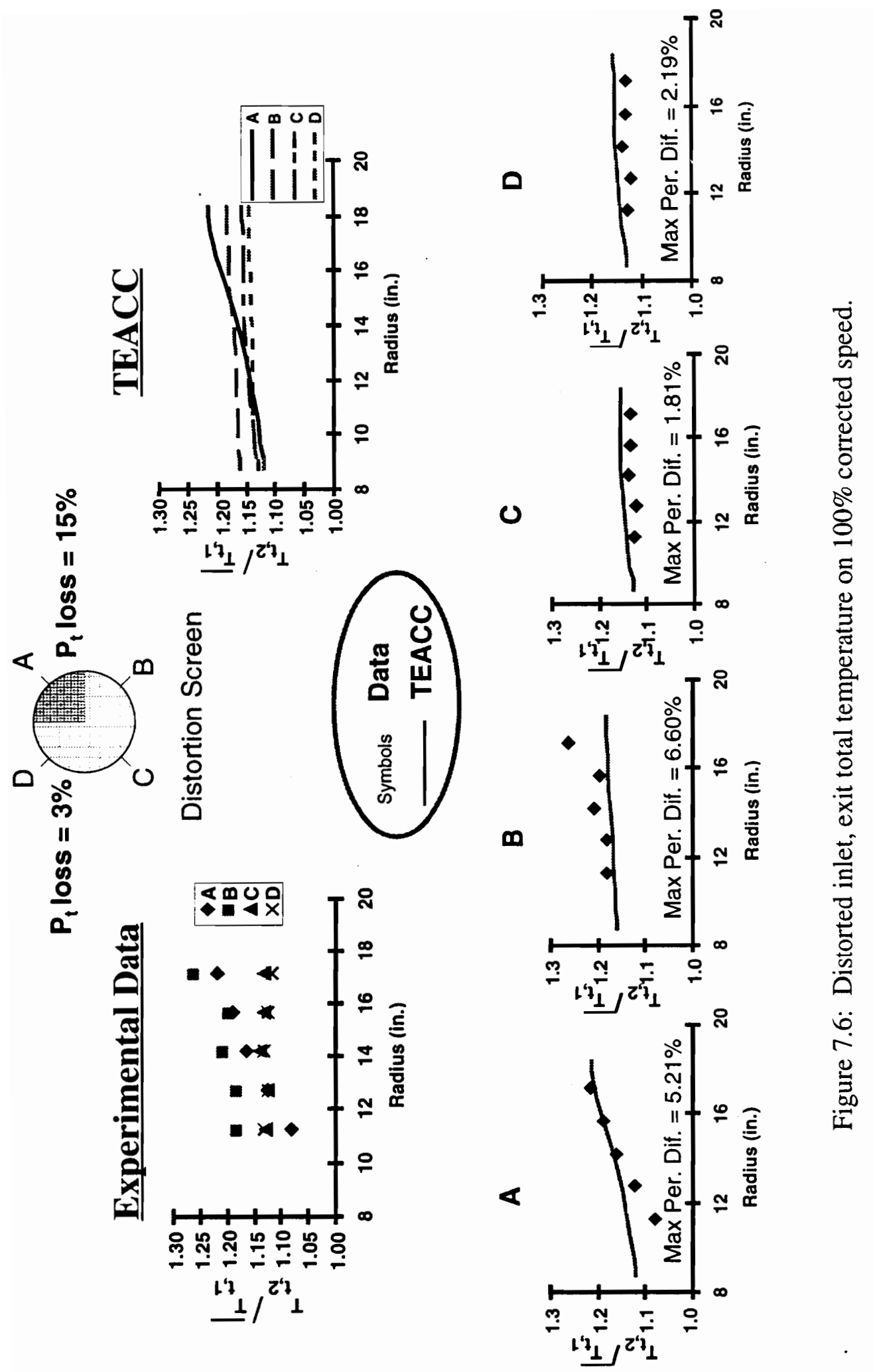


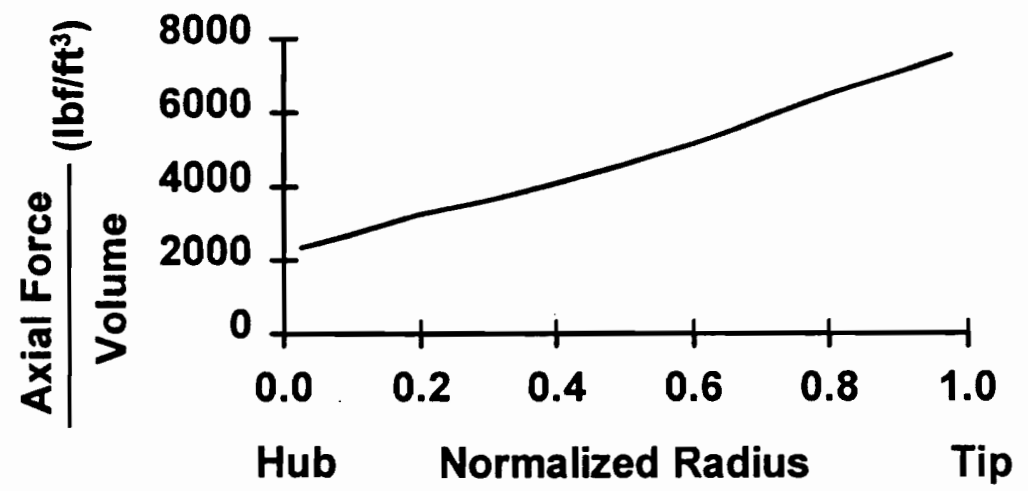

Figure 8.1: Clean inlet, axial force per volume on the $100 \%$ corrected speed near the design throttle line.

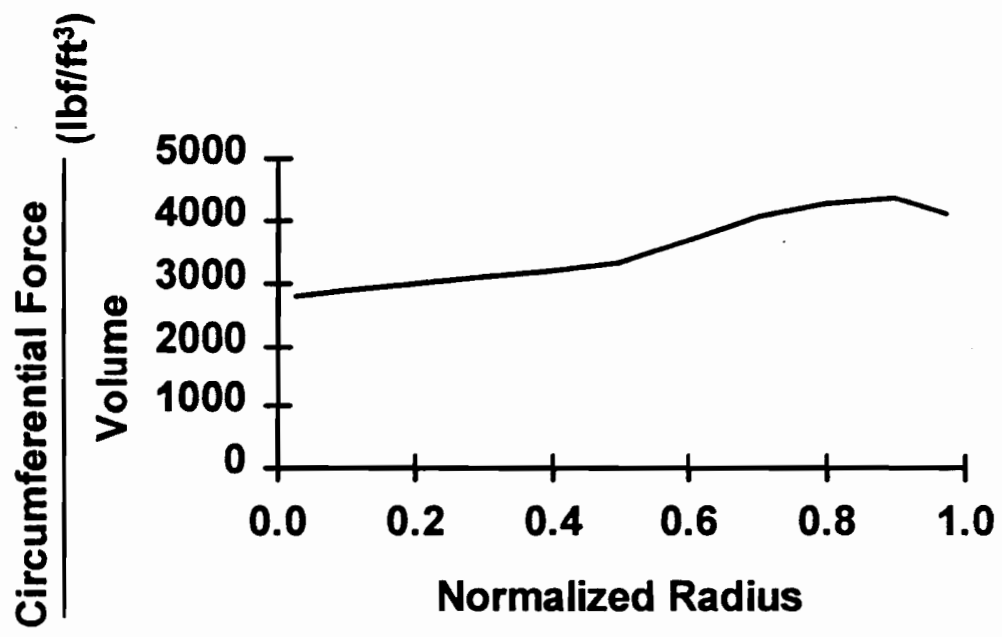

Figure 8.2: Clean inlet, circumferential force per volume on the $100 \%$ corrected speed near the design throttle line. 


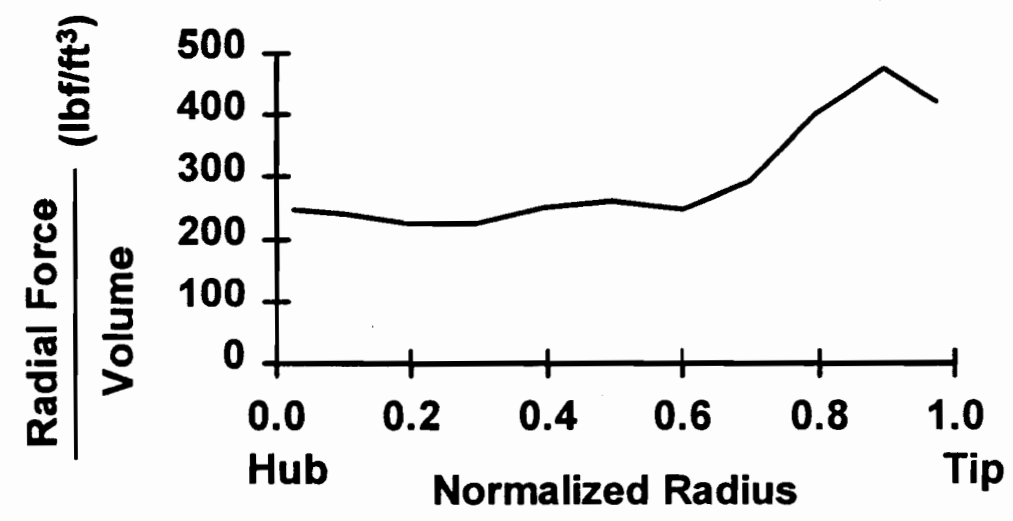

Figure 8.3: Clean inlet, radial force per volume on the $100 \%$ corrected speed near the design throttle line.

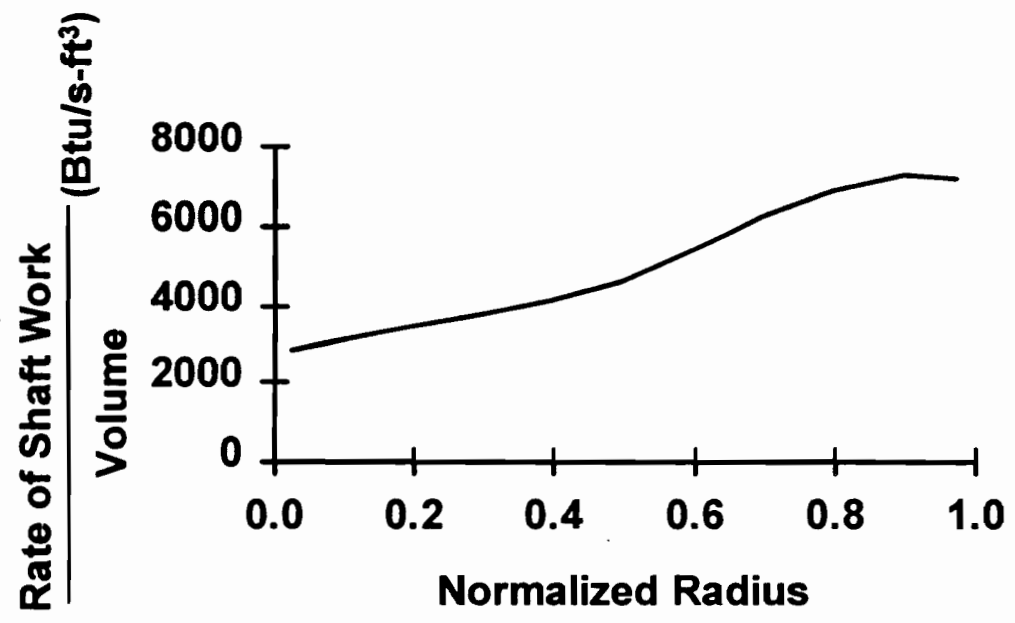

Figure 8.4: Clean inlet, rate of shaft work per volume on the $100 \%$ corrected speed near the design throttle line. 


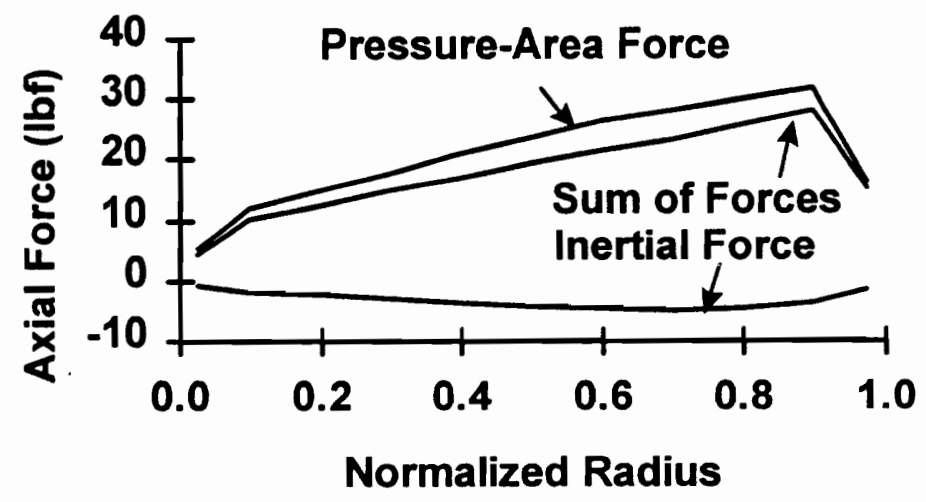

Figure 8.5: Clean inlet, radial distribution of axial force on a 15-degree circumferential segment on the $100 \%$ corrected speed line.

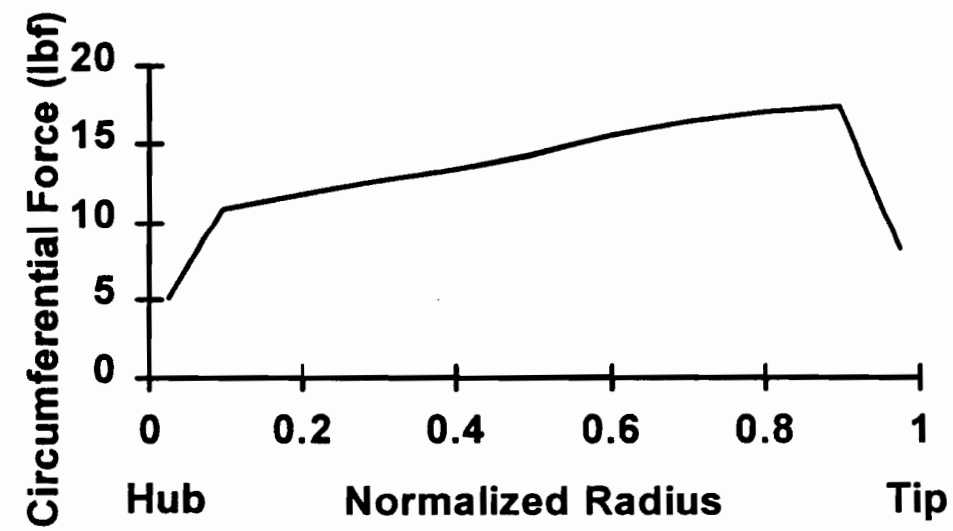

Figure 8.6: Clean inlet, radial distribution of circumferential force on a 15degree circumferential segment on the $100 \%$ corrected speed line. 


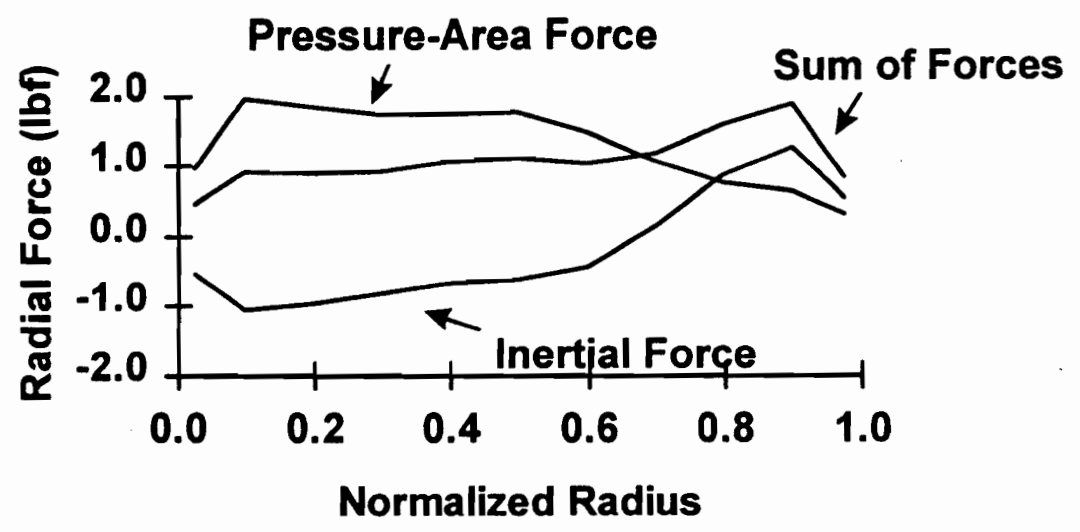

Figure 8.7: Clean inlet, radial distribution of radial force on a 15-degree circumferential segment on the $100 \%$ corrected speed line.

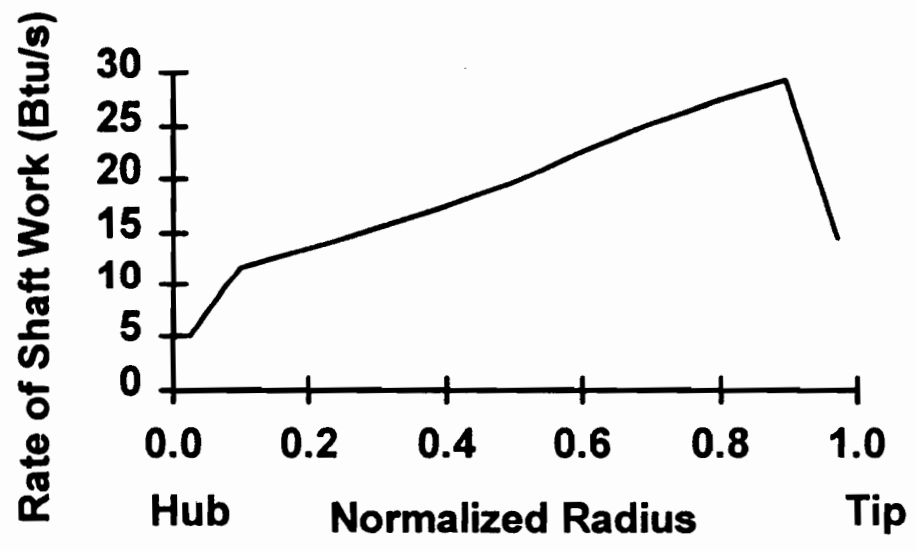

Figure 8.8: Clean inlet, rate of shaft work on a 15-degree circumferential segment on the $100 \%$ corrected speed line. 


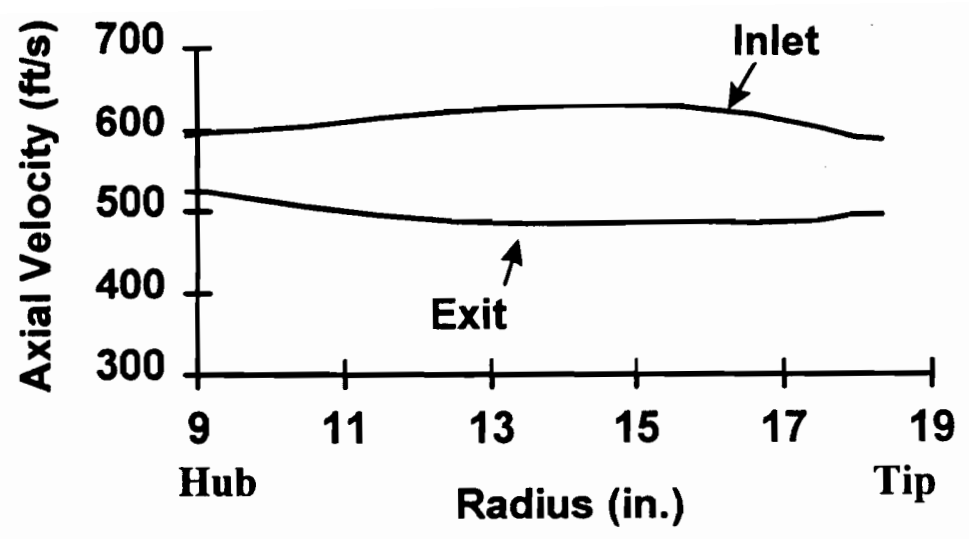

Figure 8.9: Clean inlet, inlet and exit axial velocity on the $100 \%$ corrected speed line near the throttle line.

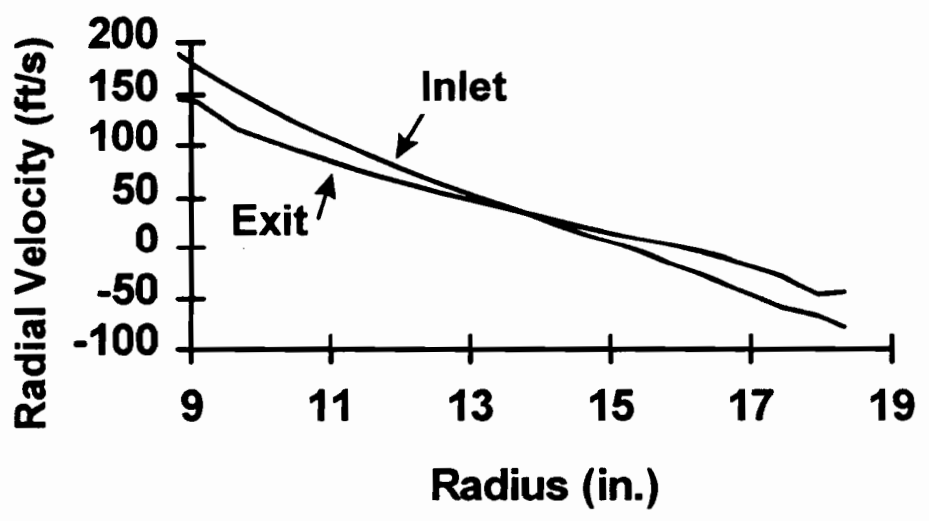

Figure 8.10: Clean inlet, inlet and exit radial velocity on the $100 \%$ corrected speed line near the throttle line. 


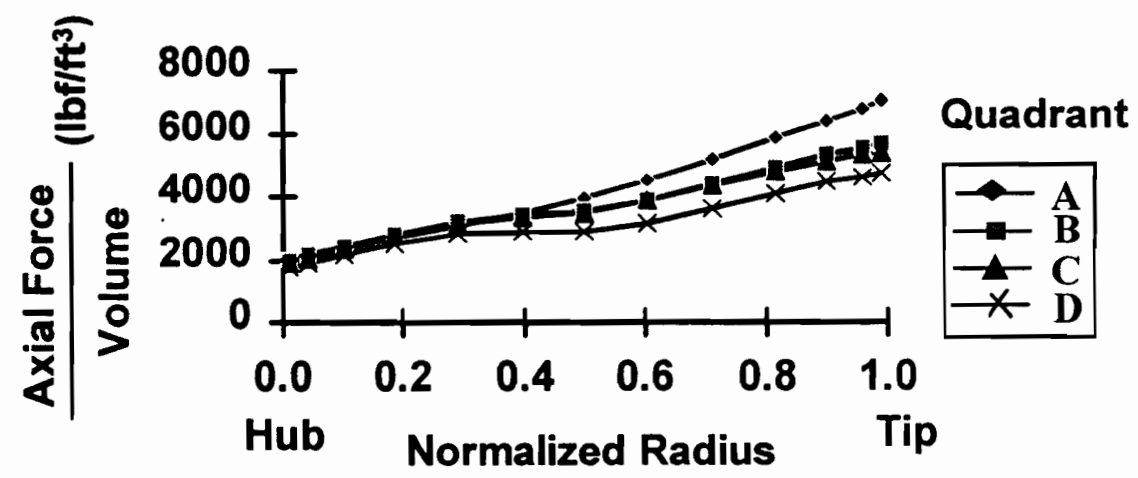

Figure 8.11: Distorted inlet, axial force per volume on the $100 \%$ corrected speed line.

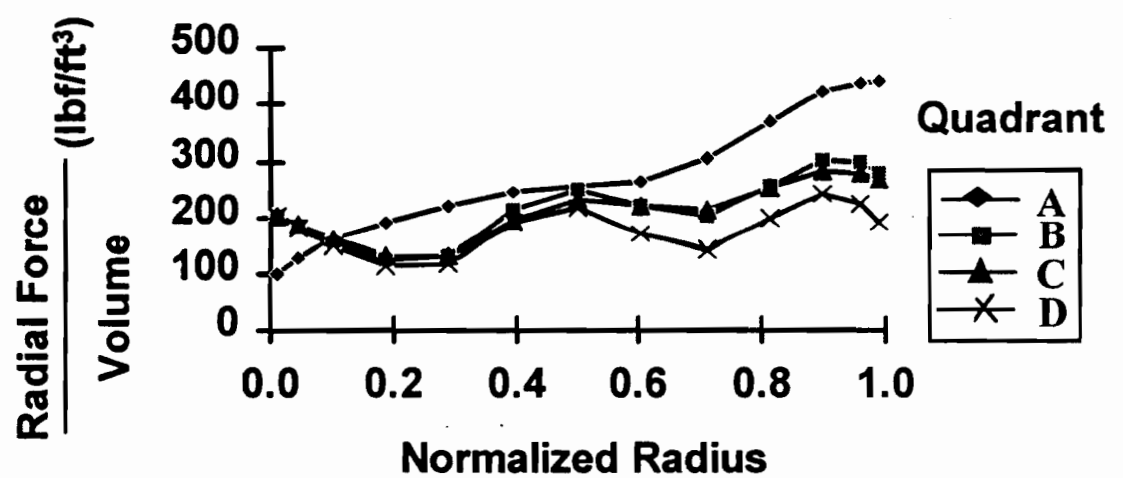

Figure 8.12: Distorted inlet, radial force per volume on the $100 \%$ corrected speed line. 


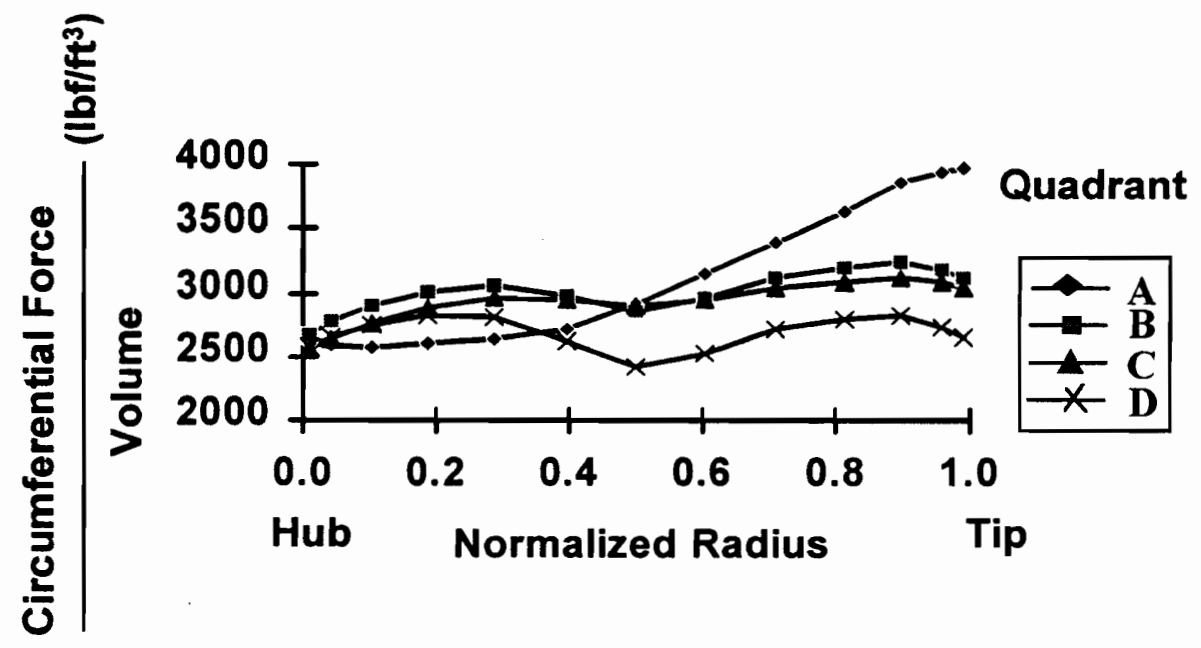

Figure 8.13: Distorted inlet, circumferential force per volume on the $100 \%$ corrected speed line.

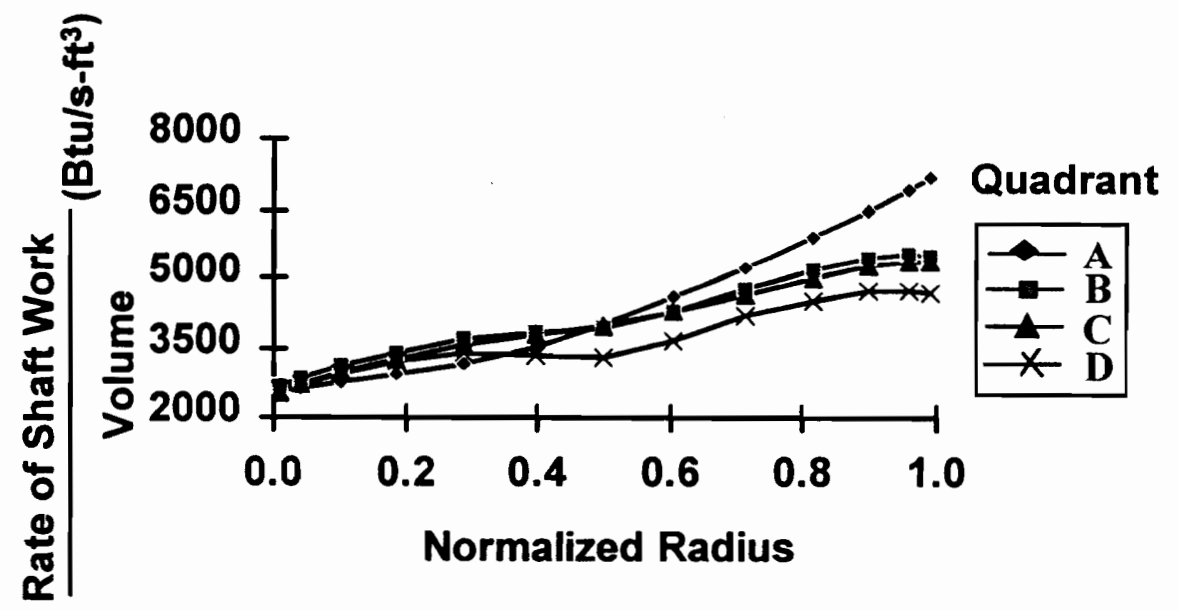

Figure 8.14: Distorted inlet, rate of shaft work per volume on the $100 \%$ corrected speed line. 


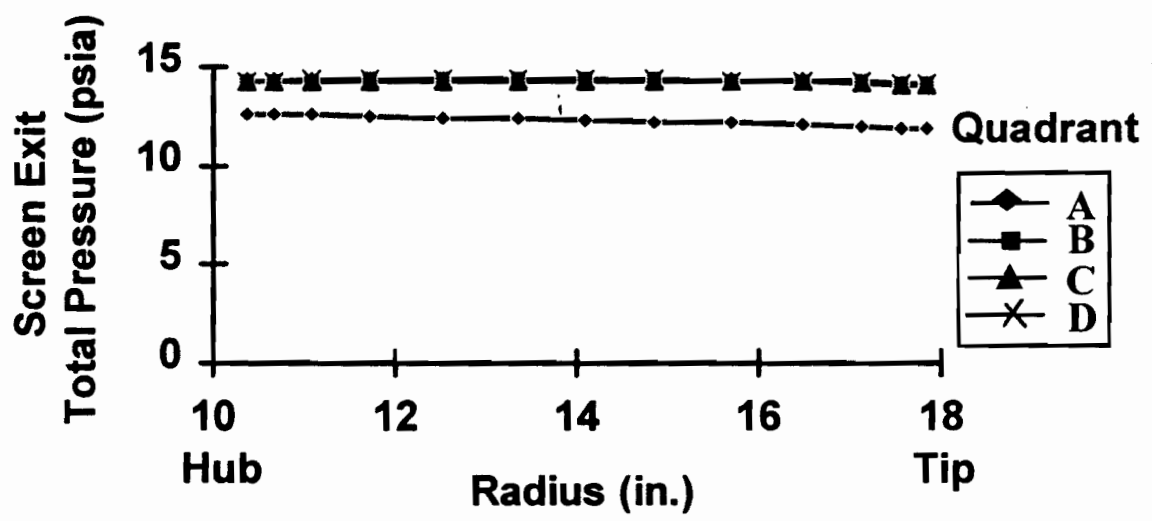

Figure 8.15: Distorted inlet, screen exit total pressure on the $100 \%$ corrected speed line.

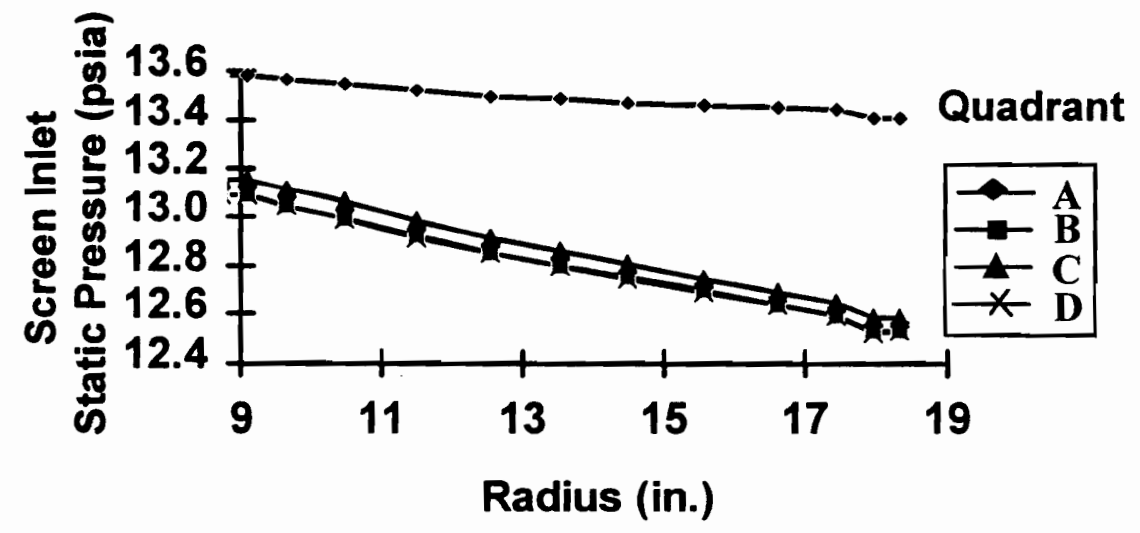

Figure 8.16: Distorted inlet, screen inlet static pressure on the $100 \%$ corrected speed line. 


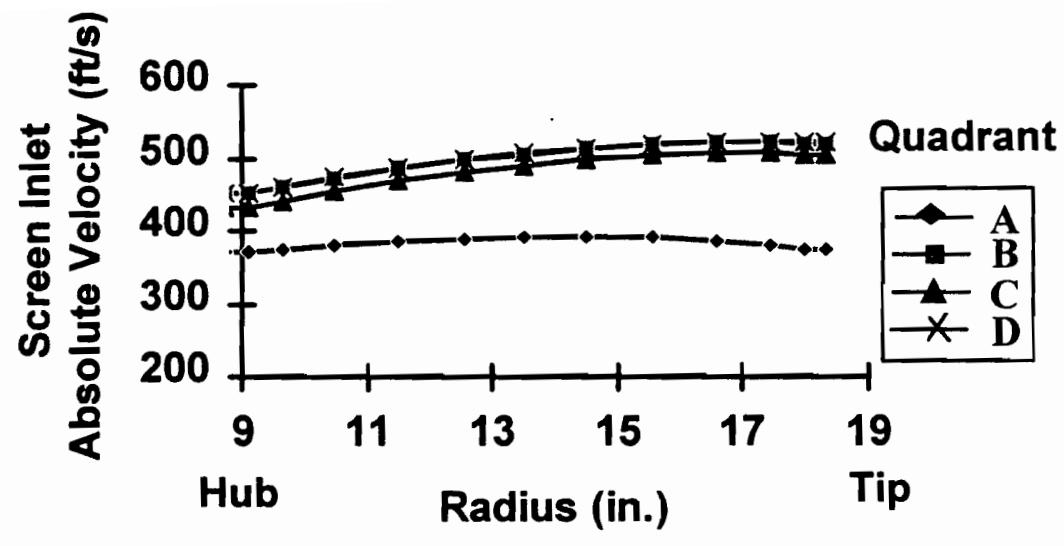

Figure 8.17: Distorted inlet, screen inlet absolute velocity on the $100 \%$ corrected speed line.

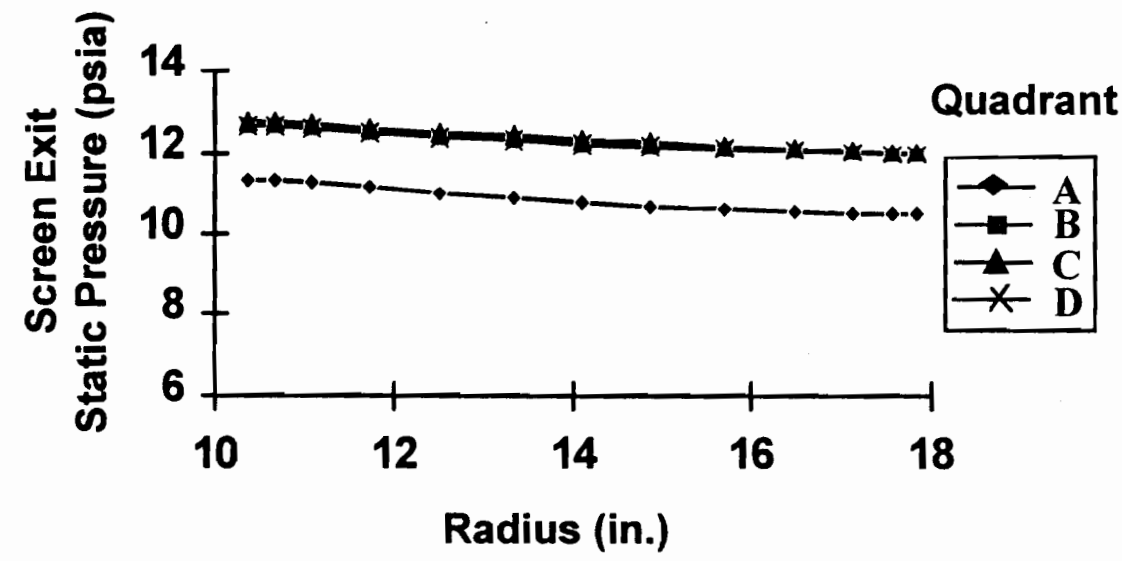

Figure 8.18: Distorted inlet, screen exit static pressure on the $100 \%$ corrected speed line. 


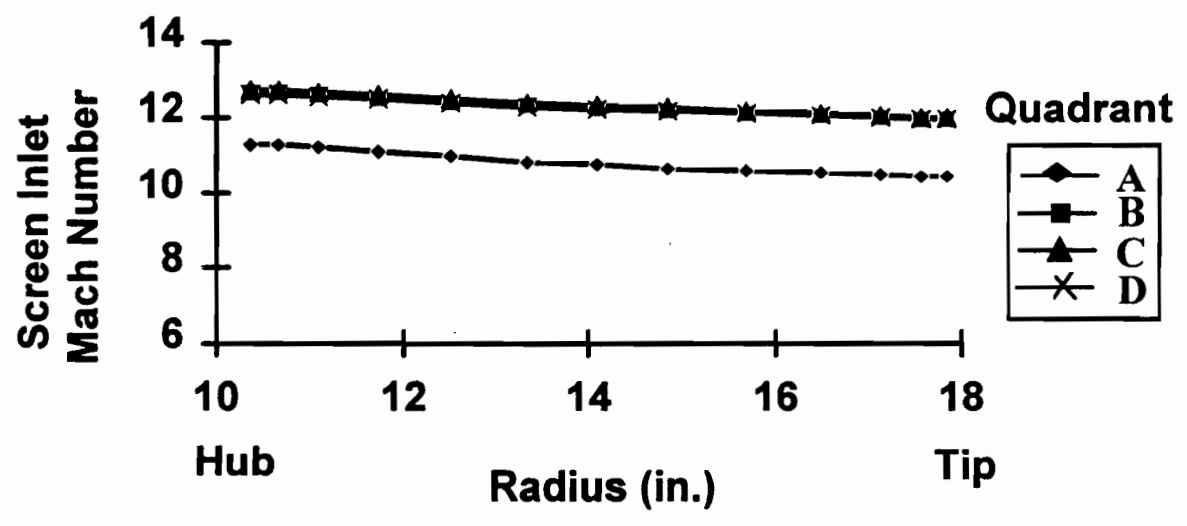

Figure 8.19: Distorted inlet, screen inlet Mach number on the 100\% corrected speed line.

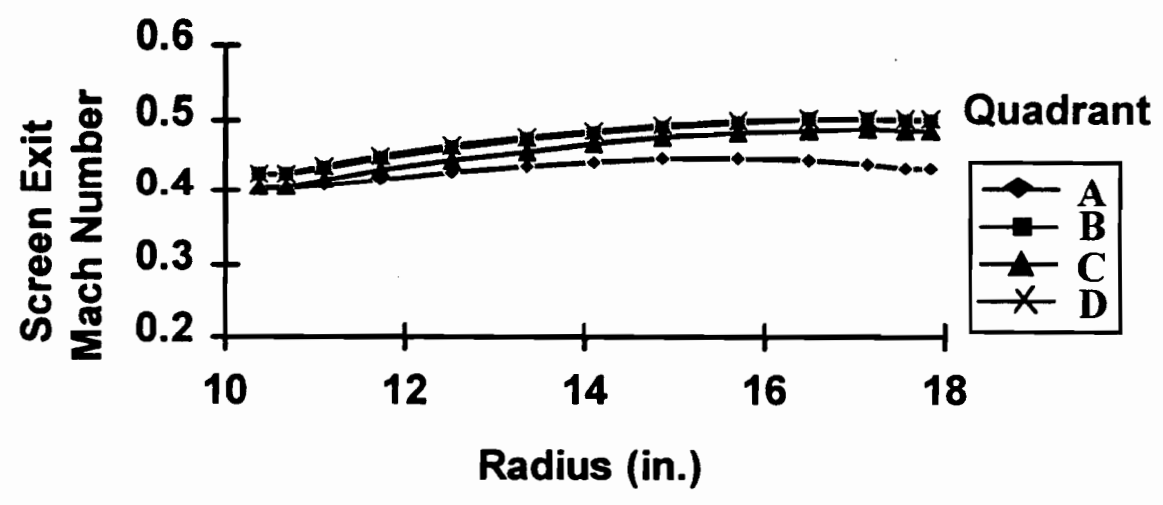

Figure 8.20: Distorted inlet, screen exit Mach number on the 100\% corrected speed line. 


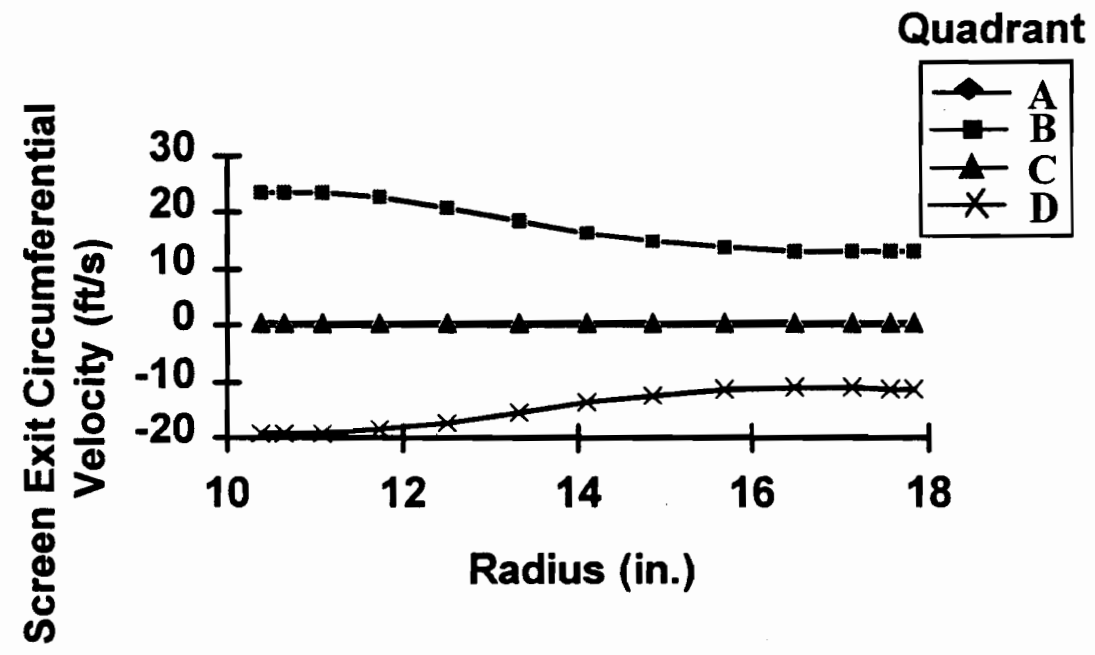

Figure 8.21: Distorted inlet, screen exit absolute circumferential velocity on the $100 \%$ corrected speed line.

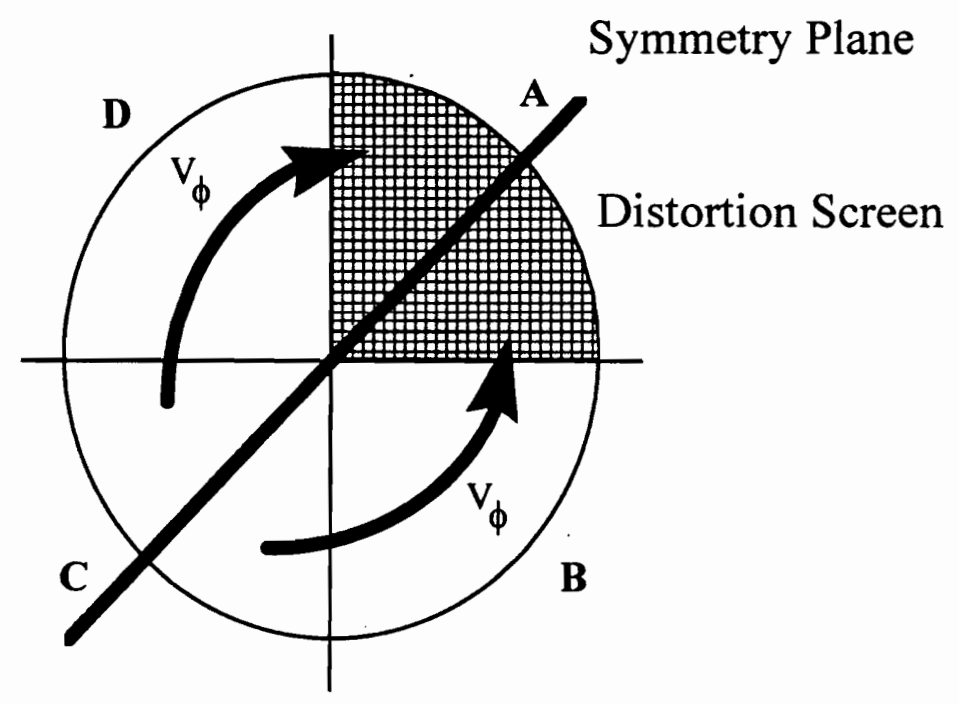

Figure 8.22: Schematic of circumferential flow about the plane of symmetry bisecting the inlet distortion. 


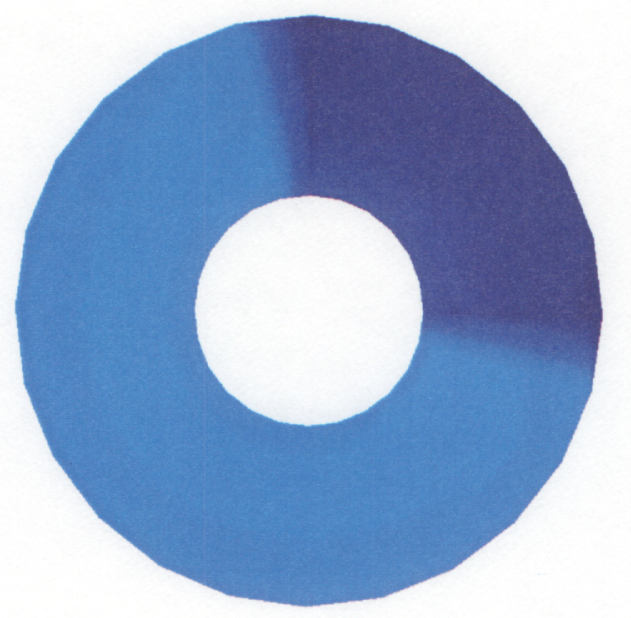

Figure 8.23: Loss in total pressure produced by the 90 -degree, one-per-revolution screen on the $100 \%$ corrected speed line.
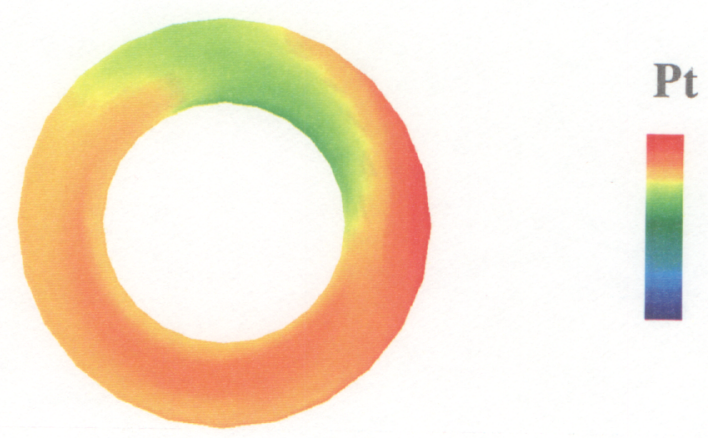

25 psia

10 psia

Figure 8.24: Exit total pressure distribution produced by 90 -degree, one-per-revolution screen on the $100 \%$ corrected speed line. 


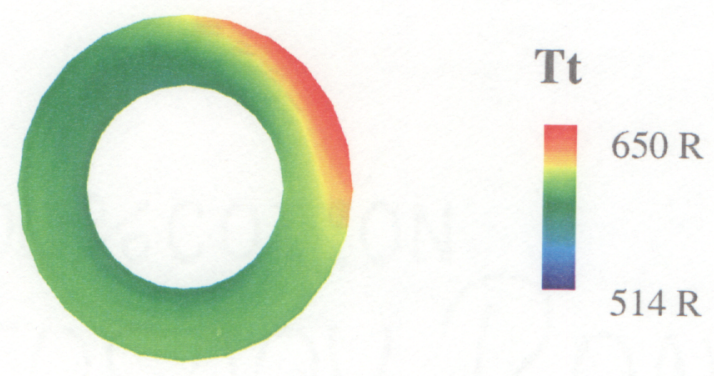

Figure 8.25: Exit total temperature distribution produced by 90 -degree, one-per-revolution screen on the $100 \%$ corrected speed line.

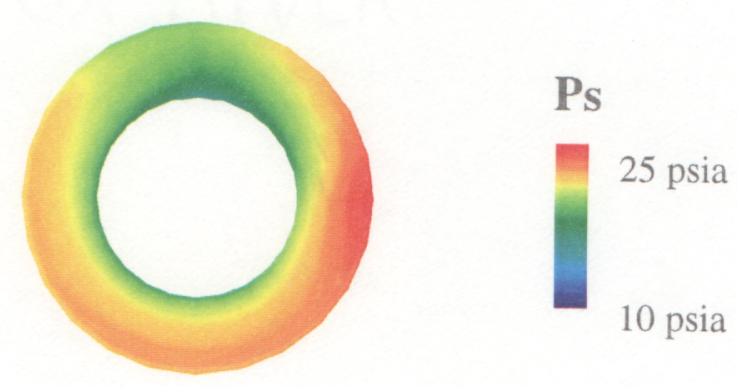

Figure 8.26: Exit static pressure distribution produced by 90-degree, one-per-revolution screen on the $100 \%$ corrected speed line.

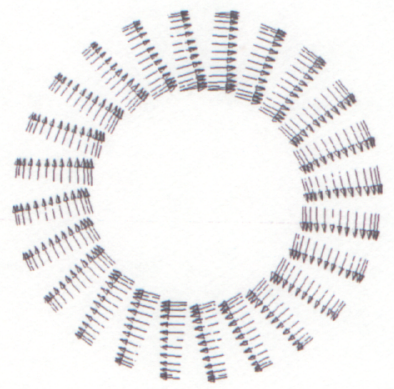

Figure 8.27: Exit absolute circumferential velocity distribution produced by 90 -degree, one-per-revolution screen on the $100 \%$ corrected speed line. 

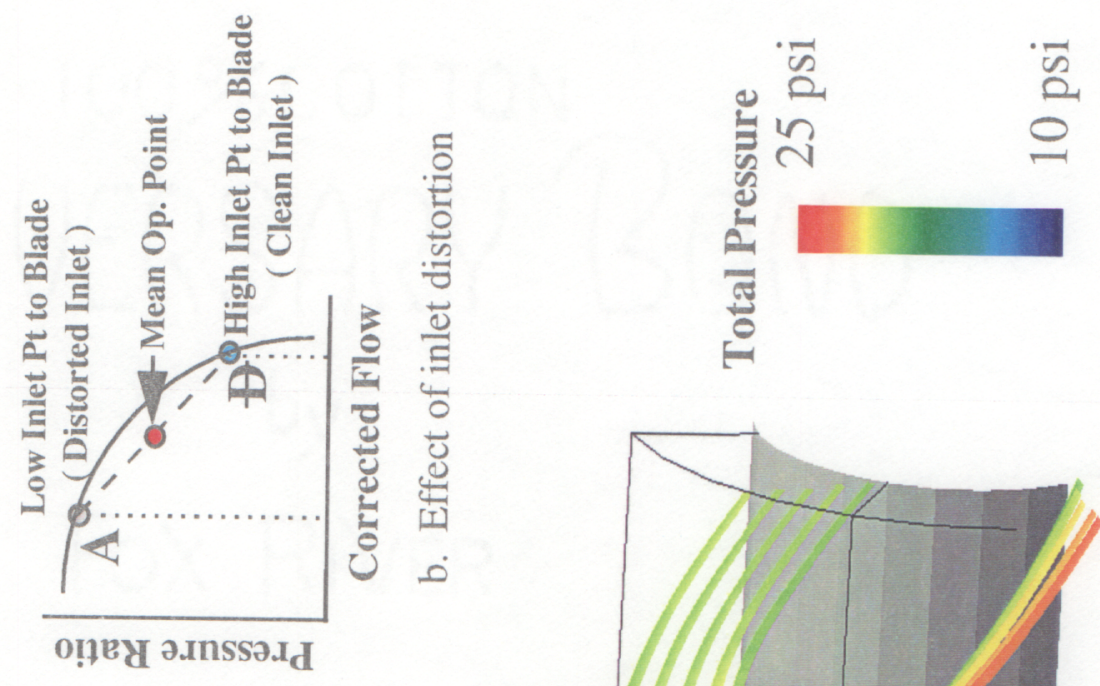

8
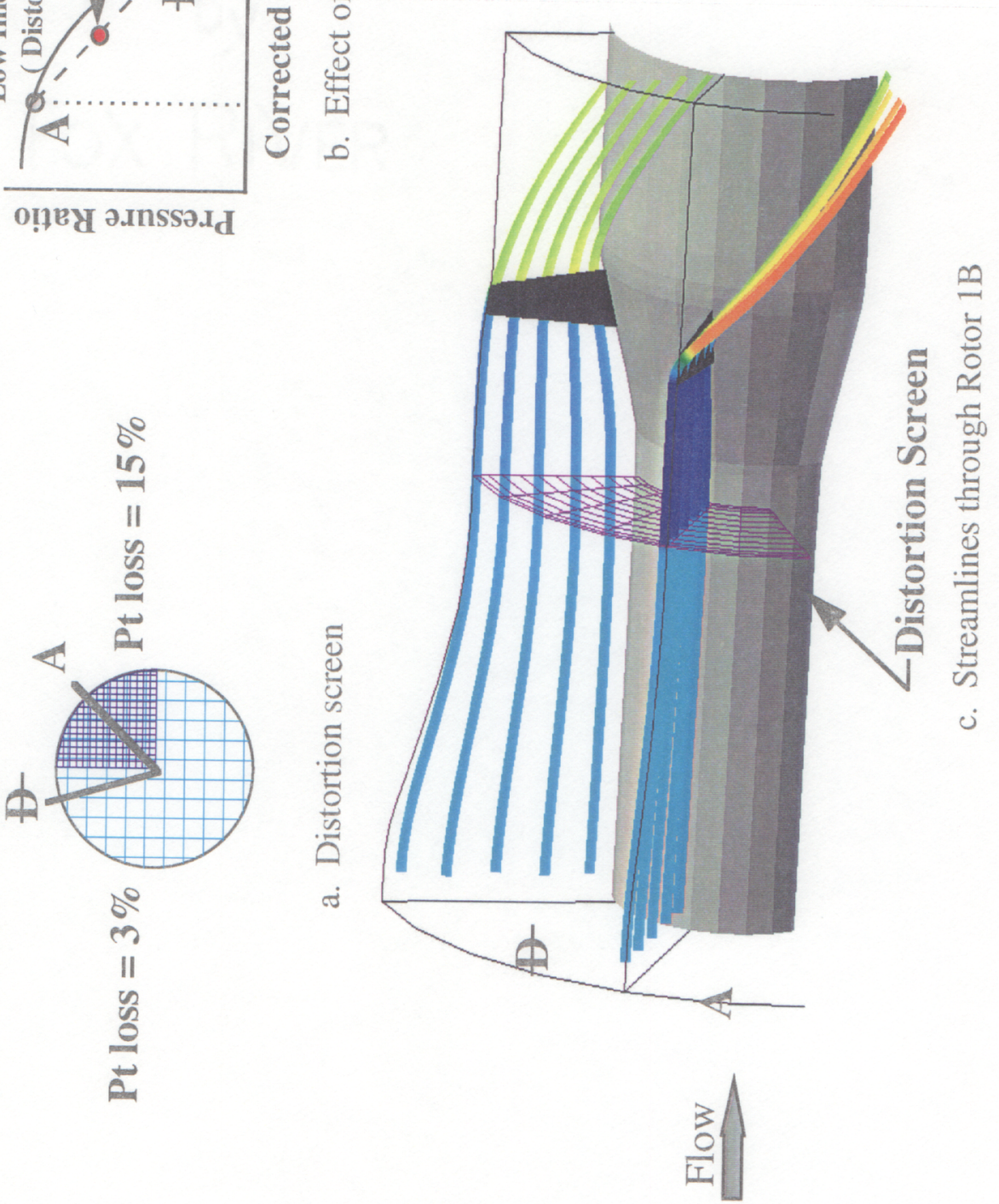

5
की
11
5
0
0 

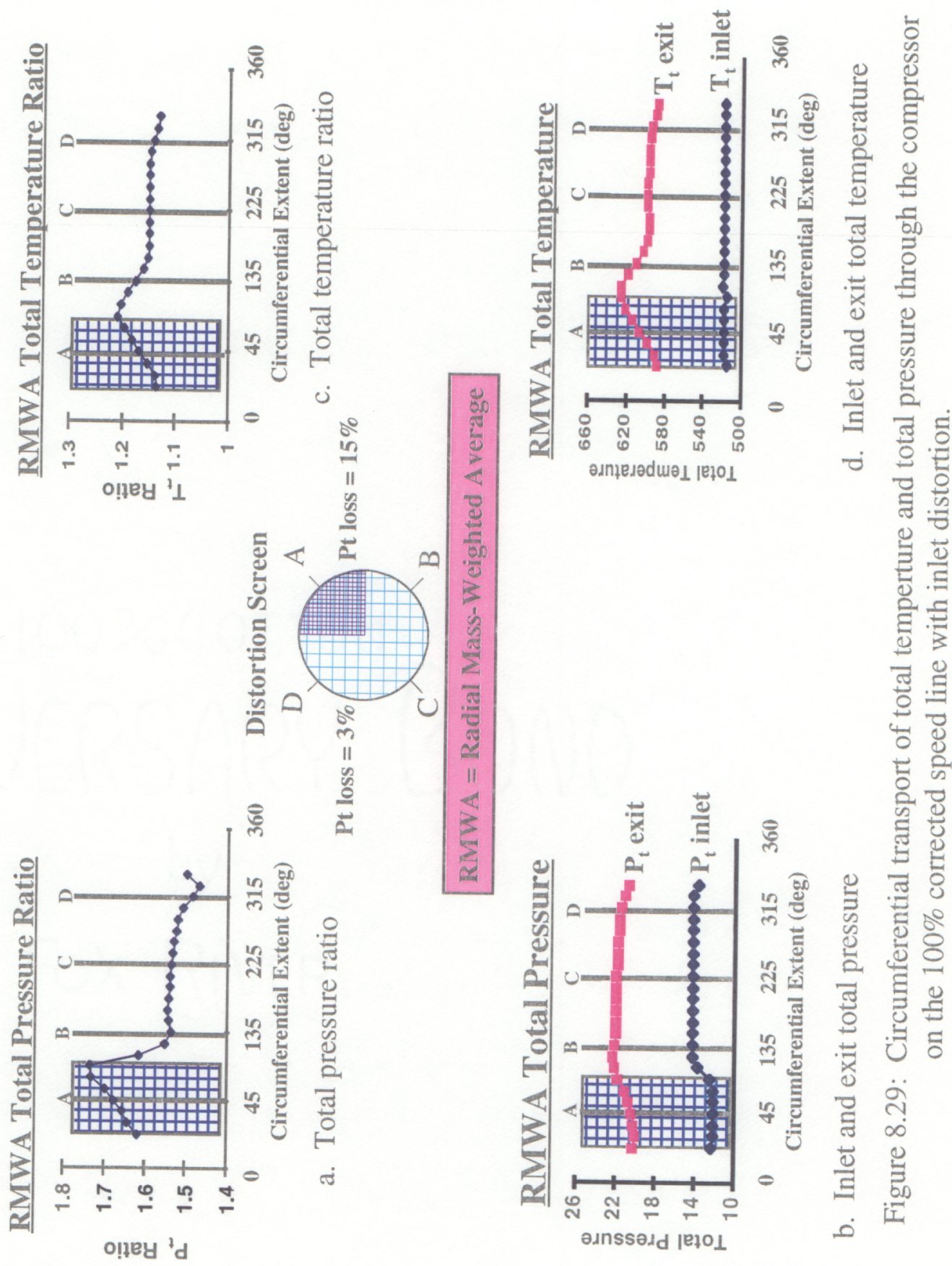


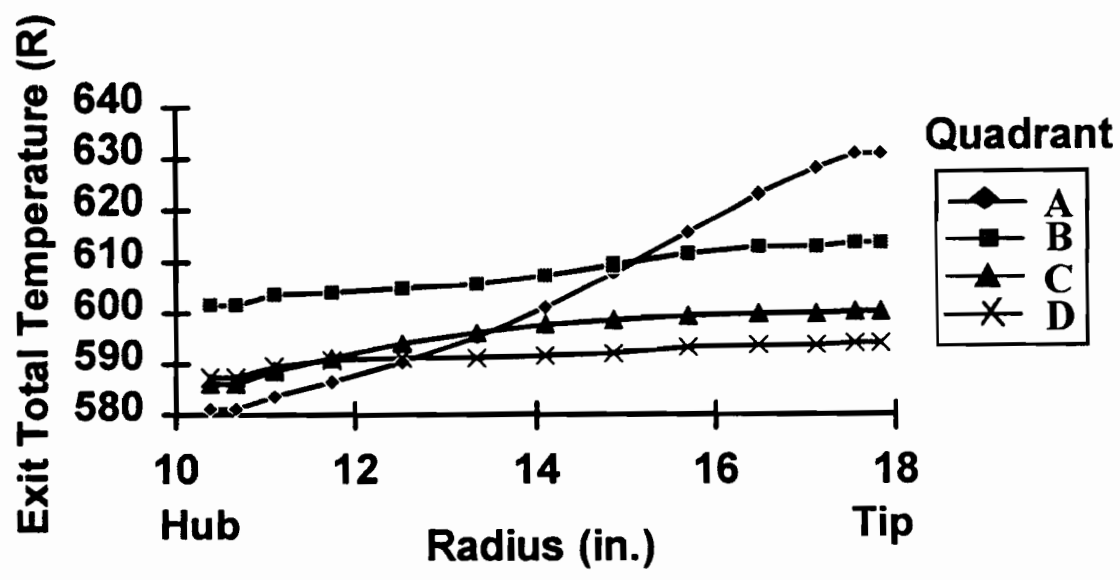

Figure 8.30: Distorted inlet, exit total temperature on the $100 \%$ corrected speed line.

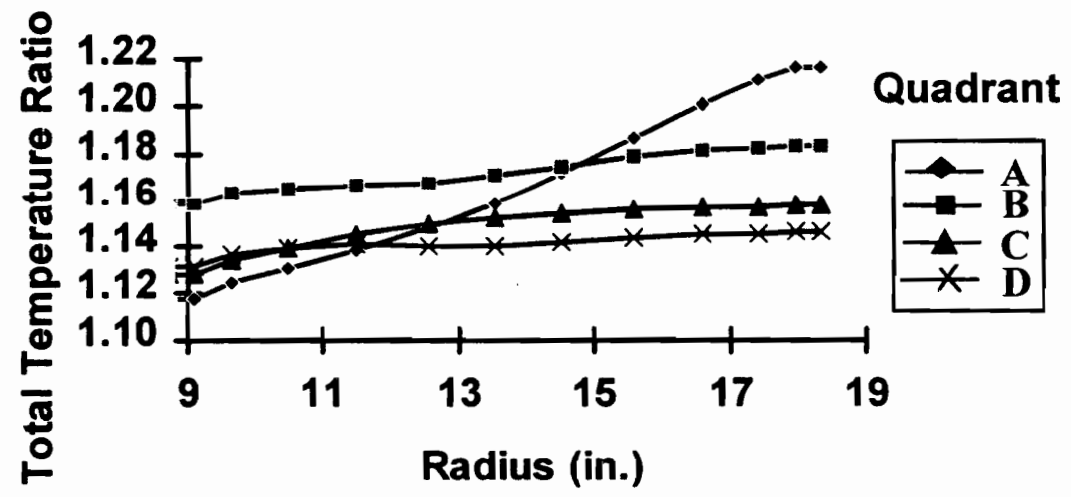

Figure 8.31: Distorted inlet, total temperature ratio on the $100 \%$ corrected speed line. 


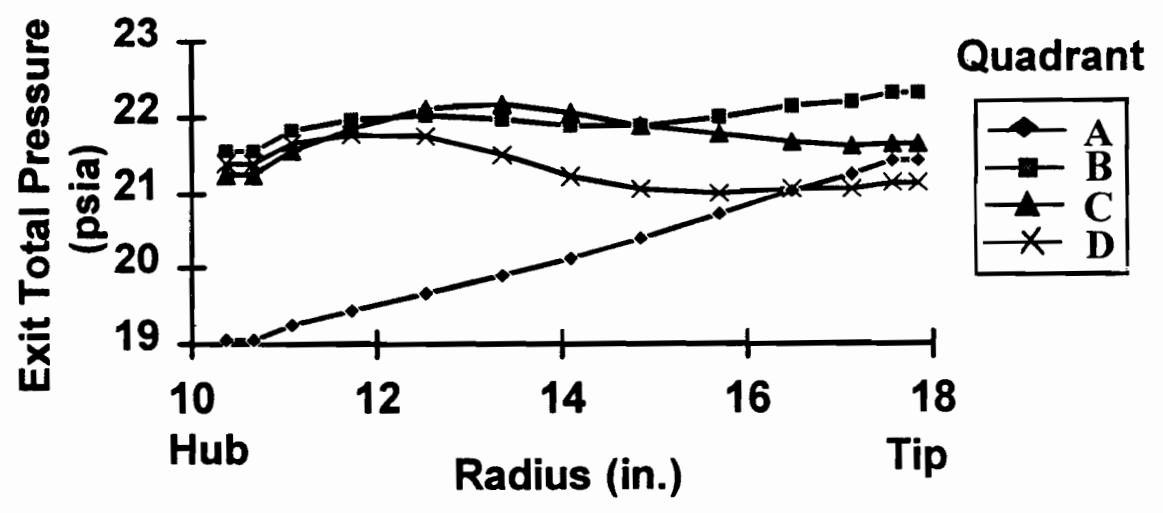

Figure 8.32: Distorted inlet, exit total pressure on the $100 \%$ corrected speed line.

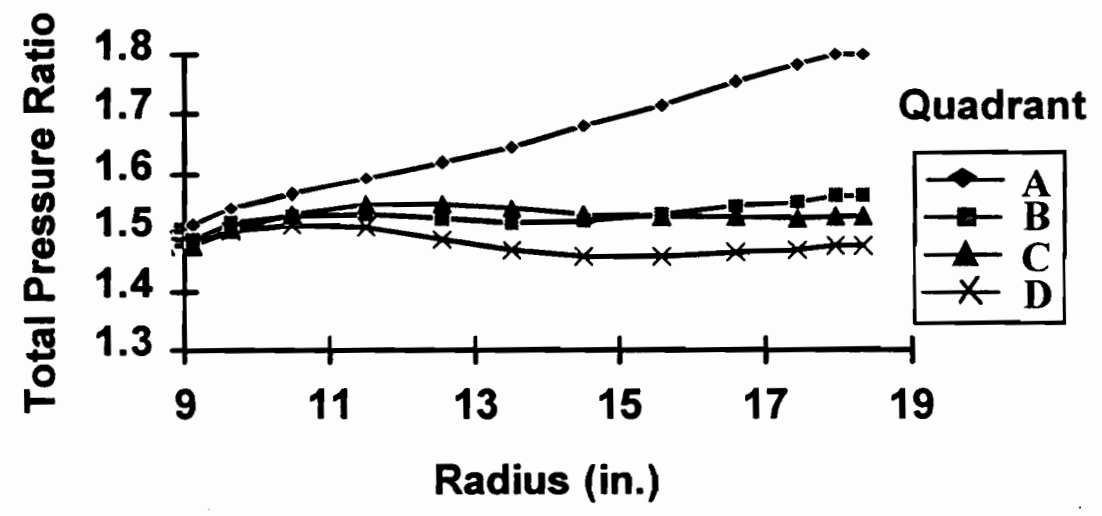

Figure 8.33: Distorted inlet, total pressure ratio on the $100 \%$ corrected speed line. 


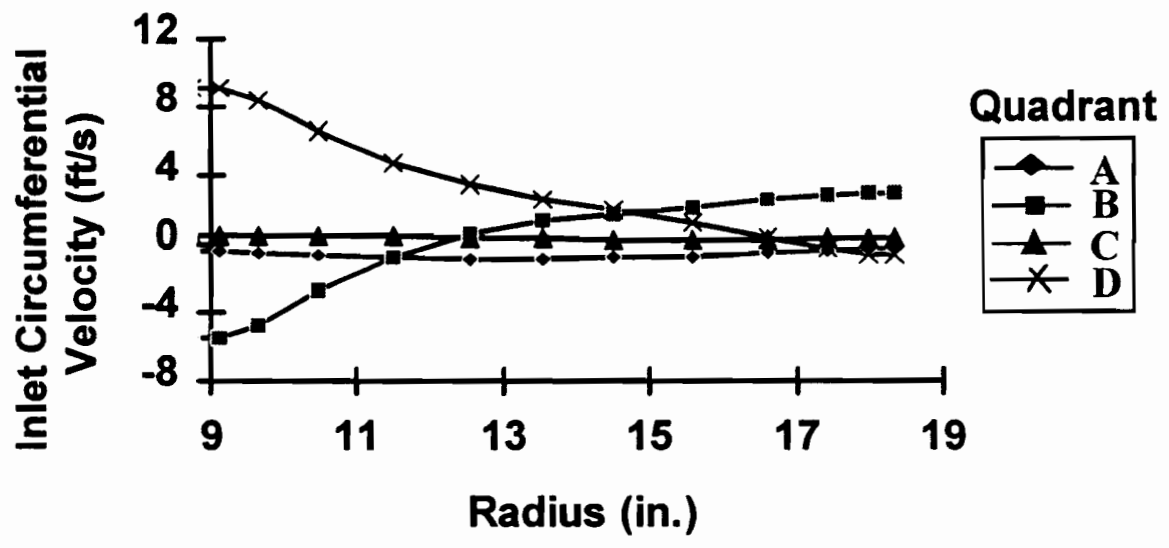

Figure 8.34: Distorted inlet, inlet circumferential velocity on the $100 \%$ corrected speed line.

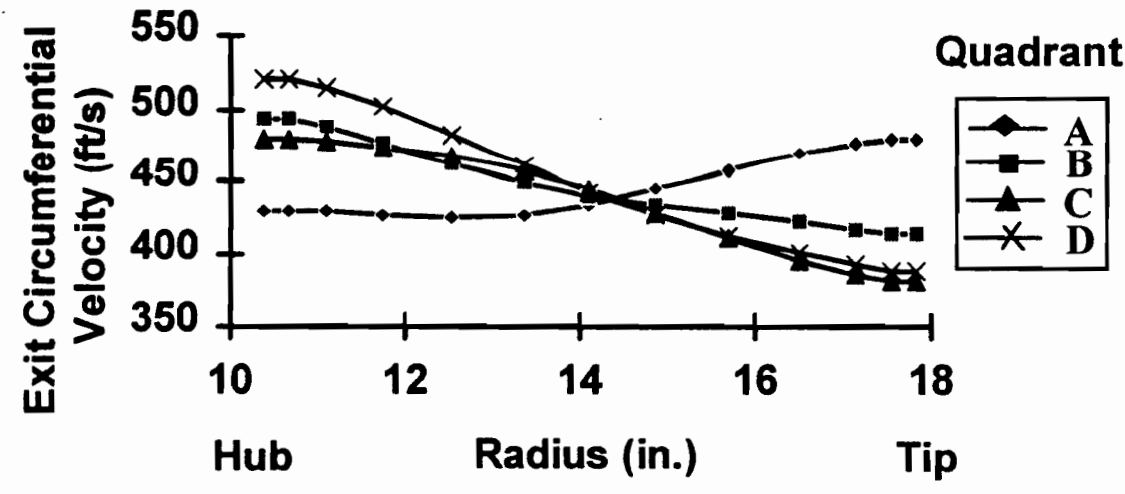

Figure 8.35: Distorted inlet, exit circumferential velocity on the $100 \%$ corrected speed line. 


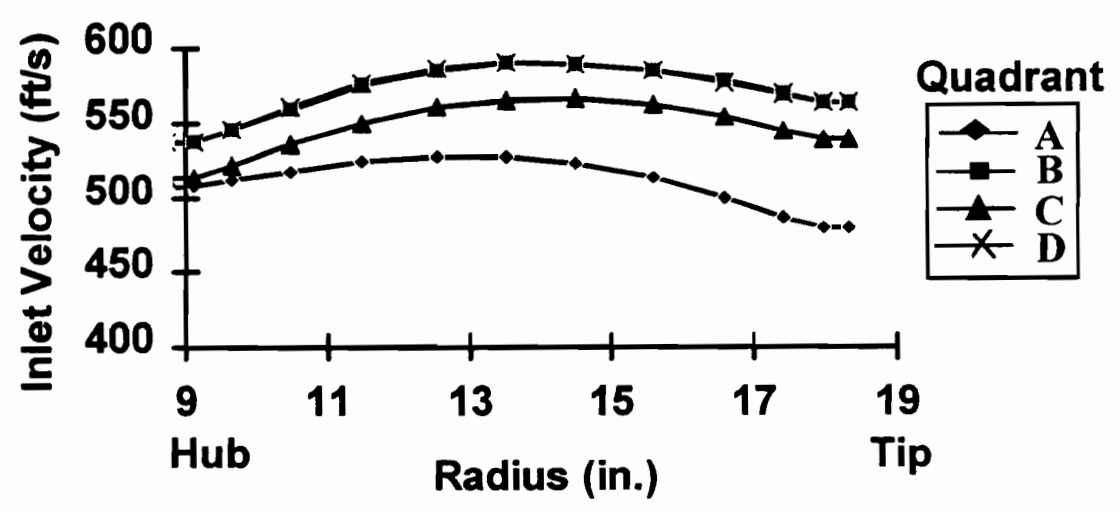

Figure 8.36: Distorted inlet, inlet velocity on the $100 \%$ corrected speed line.

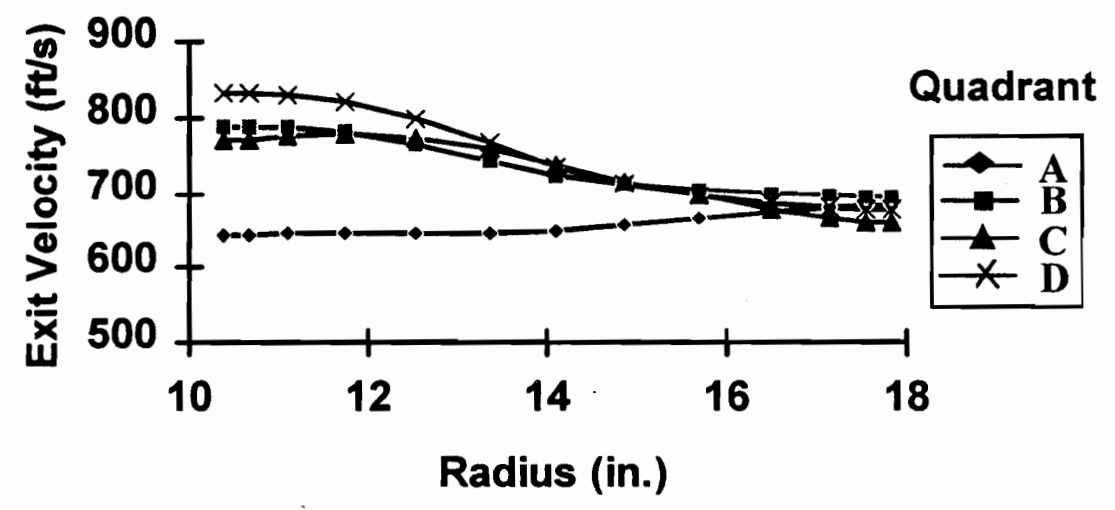

Figure 8.37: Distorted inlet, exit velocity on the $100 \%$ corrected speed line. 


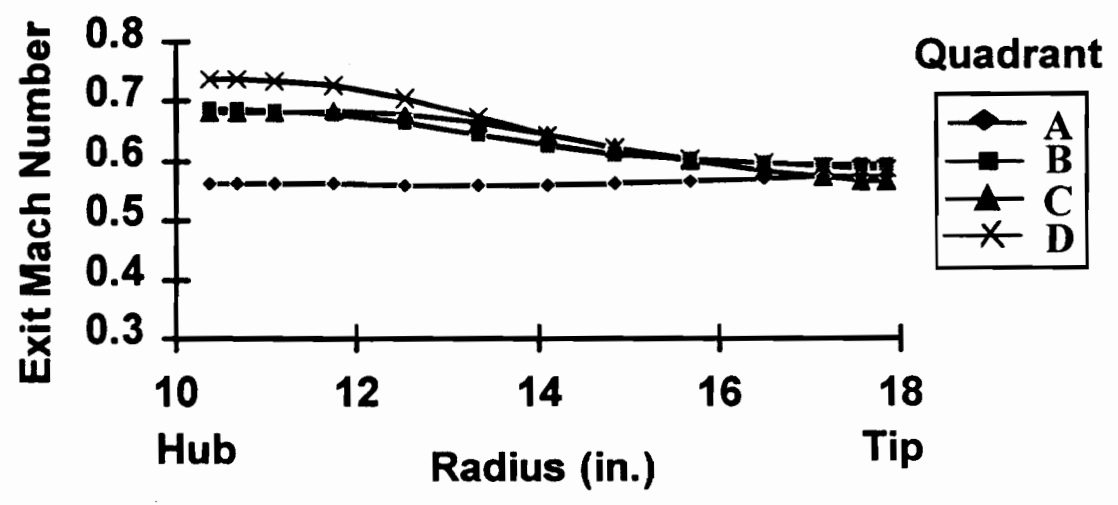

Figure 8.38: Distorted inlet, exit Mach number on the 100\% corrected speed line.

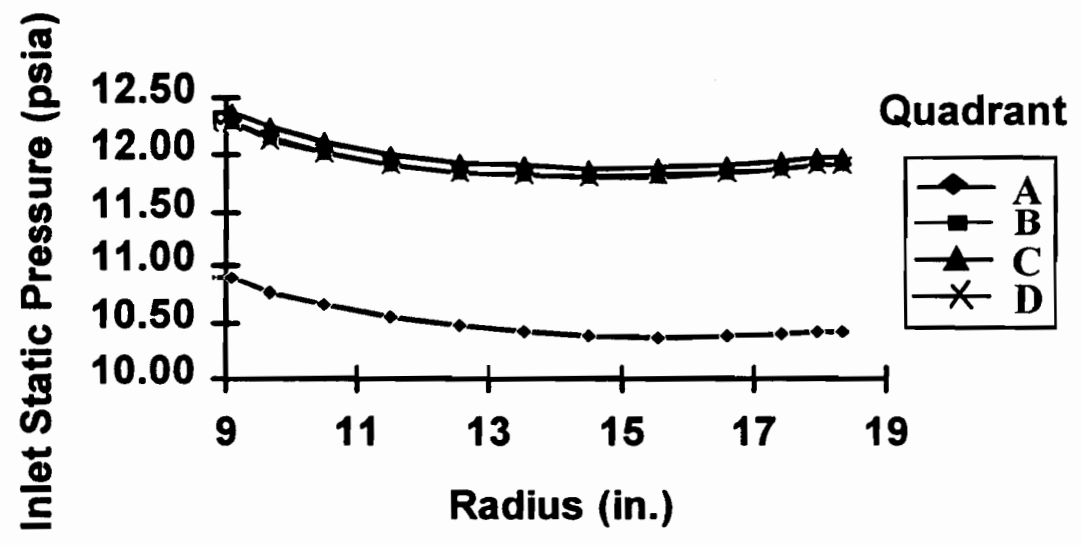

Figure 8.39: Distorted inlet, inlet static pressure on the $100 \%$ corrected speed line. 


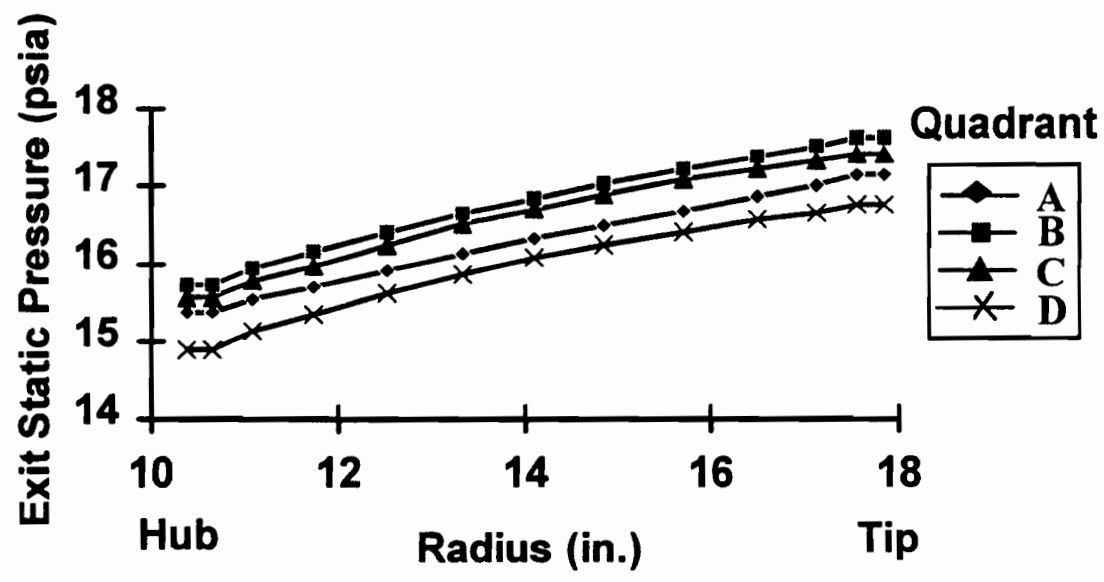

Figure 8.40: Distorted inlet, exit static pressure on the $100 \%$ corrected speed line.

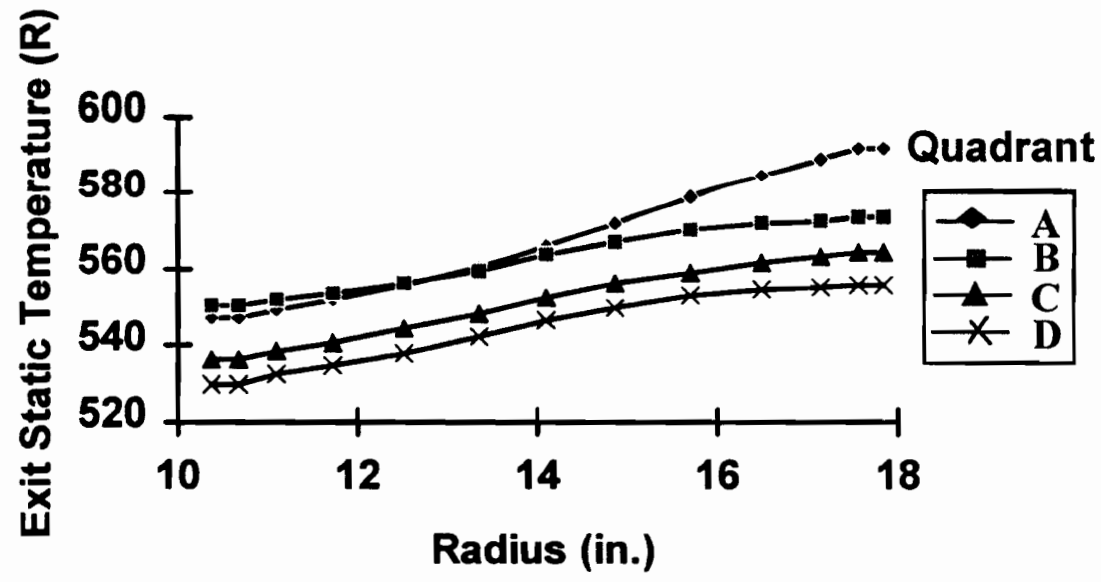

Figure 8.41: Distorted inlet, exit static temperature on the $100 \%$ corrected speed line. 


\section{Appendix C - Review of Streamline Curvature Code Development}

The SLCC provides a mechanism to produce turbomachinery source terms by solving a form of the steady-state Euler equations as described in Chapter 3. The derivation of these equations and solution procedure were developed completely by Hearsey (1970). Only the governing equations and the primary assumptions are provided here as a review.

The Navier-Stokes equations can be greatly simplified by assuming steady state, no body forces, no heat transfer, axial symmetry, and inviscid flow to produce the following set of equations (nomenclature in Section C.1):

continuity,

$$
\frac{\partial\left(\rho \mathrm{r} \lambda \mathrm{V}_{\mathrm{r}}\right)}{\partial \mathrm{r}}+\frac{\partial\left(\rho \mathrm{r} \lambda \mathrm{V}_{\mathrm{z}}\right)}{\partial \mathrm{z}}=0
$$

circumferential momentum, $\mathrm{V}_{\mathrm{r}} \frac{\partial \mathrm{V}_{\mathrm{w}}}{\partial \mathrm{r}}+\mathrm{V}_{\mathrm{z}} \frac{\partial \mathrm{V}_{\mathrm{w}}}{\partial \mathrm{z}}+\frac{\mathrm{V}_{\mathrm{r}} \mathrm{V}_{\mathrm{w}}}{\mathrm{r}}=0$,

axial momentum,

$$
\mathrm{V}_{\mathrm{r}} \frac{\partial \mathrm{V}_{\mathrm{z}}}{\partial \mathrm{r}}+\mathrm{V}_{\mathrm{z}} \frac{\partial \mathrm{V}_{\mathrm{z}}}{\partial \mathrm{z}} \quad=-\frac{1}{\rho} \frac{\partial \mathrm{p}}{\partial \mathrm{z}}, \text { and }
$$

radial momentum,

$$
\mathrm{V}_{\mathrm{r}} \frac{\partial \mathrm{V}_{\mathrm{r}}}{\partial \mathrm{r}}+\mathrm{V}_{\mathrm{z}} \frac{\partial \mathrm{V}_{\mathrm{r}}}{\partial \mathrm{z}}-\frac{\mathrm{V}_{\mathrm{w}}^{2}}{\mathrm{r}}=-\frac{1}{\rho} \frac{\partial \mathrm{p}}{\partial \mathrm{r}}
$$

From the assumption of axial symmetry, the circumferential momentum equation can be simplified along a streamline by

$$
\frac{\mathrm{d}\left(\mathrm{rV}_{\mathrm{w}}\right)}{\mathrm{dm}}=0
$$


which means angular momentum is conserved along a streamline. The ideal gas equation of state with constant properties is used. At every axial station, three closure relations must be specified for each streamline: entropy, enthalpy (total pressure and total temperature), and swirl velocity. With these specified quantities and the above set of equations, streamlines can be resolved though the flow field.

For duct flow away from compressor blades, the three closure variables, total temperature, total pressure, and swirl velocity are easily specified at each axial station from knowledge of the previous axial station as follows:

$$
\begin{aligned}
& \mathrm{Tt}_{2}=\mathrm{Tt}_{1} \\
& \mathrm{Pt}_{2}=\mathrm{Pt}_{1} \\
& \mathrm{~V}_{\mathrm{w} .2}=\mathrm{V}_{\mathrm{w} .1}\left(\frac{\mathrm{r}_{1}}{\mathrm{r}_{2}}\right)
\end{aligned}
$$

Then the solution technique, which solves momentum and continuity simultaneously, calculates the current location of the streamlines. When the streamlines are known, the inlet and exit absolute velocities, along with all other flow-field variables, can be calculated.

However, the three closure relations, total temperature, total pressure, and swirl velocity at the exit of the bladed regions, are more complicated, requiring the specification of empirical relations. The technique used to cross the bladed region begins by calculating the relative inlet flow field to the blades from static quantities and inlet velocity triangles. Two empirical quantities are specified in the relative reference frame to represent the effect of the blades. They are the relative total pressure loss coefficient 
based upon inlet dynamic head, $\bar{\omega}^{\prime}=\frac{\left(P_{t, 2}^{\prime}\right)_{i d}-P_{t, 2}^{\prime}}{P_{t, 1}^{\prime}-P_{1}}$, and relative exit flow angle, $\beta_{2}$. From exit velocity triangles and exit streamline angles, the meridional velocity, wheel speed, and streamline angles are known at the exit radius. When the relative exit flow angle is specified, the first of the unknowns, absolute swirl velocity, can be calculated from:

$$
\begin{aligned}
& \tan \beta_{2}=\frac{\mathrm{W}_{\mathrm{w}, 2}}{\mathrm{~V}_{\mathrm{m}, 2}} \\
& \cos \phi_{2}=\frac{\mathrm{V}_{\mathrm{z}, 2}}{\mathrm{~V}_{\mathrm{m}, 2}} \\
& \mathrm{~V}_{\mathrm{w}, 2}=\mathrm{V}_{\mathrm{m}, 2} \tan \beta_{2} \cos \phi_{2}+\mathrm{U}_{2}
\end{aligned}
$$

The exit total temperature can now be calculated from the Euler turbine equation (Cohen, 1987), since the exit swirl velocity is now known, as follows:

$$
\mathrm{T}_{\mathrm{t}, 2}=\mathrm{T}_{\mathrm{t}, \mathrm{l}}+\mathrm{U}_{2}\left[\mathrm{~V}_{\mathrm{w}, 2}-\frac{\mathrm{r}_{1}}{\mathrm{r}_{2}} \mathrm{~V}_{\mathrm{w}, 1}\right] \frac{\sigma}{\mathrm{C}_{\mathrm{p}}}
$$

Finally, the exit absolute total pressure is calculated from (1) isentropic relationships, (2) rothalpy equal to a constant, and (3) the specified relative total pressure loss coefficient from

$$
P_{t, 2}=P_{t, 1}\left(\frac{T_{t, 2}}{T_{t, 1}}\right)^{\frac{\gamma}{\gamma-1}}\left[1-\left(\frac{P_{t, 1}^{\prime}}{P_{t, 2}^{\prime}}\right)_{\text {id }} \bar{\omega}\left(1-\left(\frac{1}{1+\frac{(\gamma-1)}{2}\left(M_{1}^{\prime}\right)^{2}}\right)^{\frac{\gamma}{\gamma-1}}\right]\right]
$$


where $\quad\left(\frac{\mathrm{P}_{\mathrm{t}, 1}^{\prime}}{\mathrm{P}_{\mathrm{t}, 2}^{\prime}}\right)_{\mathrm{id}}=\left\{1+\frac{(\gamma-1)}{2} \mathrm{M}_{\mathrm{T}, \mathrm{l}}^{\prime}\left[\left(\frac{\mathrm{r}_{2}}{\mathrm{r}_{1}}\right)^{2}-1\right]\right\}^{\frac{-\gamma}{\gamma-1}}$

The continuity and momentum equation can now be solved iteratively for the new streamline locations.

The technique for using the original SLCC described in this appendix is explained in Section 3.2.1. A modification of the SLCC to operate only across a bladed region is described in Section 3.2.2, where the boundary conditions are obtained from the TEACC flow field on either side of the bladed region and updated each time step. The SLCC is also used in its original form to supply initial conditions to the TEACC flow field as defined in Section 3.5.

\section{C.1 Nomenclature for Appendix C}

$\begin{array}{ll}\text { Symbol } & \text { Definition } \\ \mathrm{c}_{\mathrm{p}} & \text { Constant pressure specific heat } \\ \mathrm{m} & \text { Meridional direction } \\ \mathrm{M}_{\mathrm{T}} & \begin{array}{l}\text { Ratio of the outlet wheel speed to the inlet relative stagnation } \\ \text { velocity speed of sound }\end{array} \\ \mathrm{P} & \text { Mach number } \\ \mathrm{r} & \text { Pressure } \\ \mathrm{T} & \text { Temperature } \\ \mathrm{U} & \text { Wheel speed }\end{array}$




$\begin{array}{ll}\text { Symbol } & \text { Definition } \\ \text { V } & \text { Absolute velocity } \\ \text { W } & \text { Circumferential direction } \\ \text { W } & \text { Relative velocity } \\ \text { z } & \text { Axial direction } \\ \beta & \text { Relative flow angle } \\ \rho & \text { Density } \\ \lambda & \text { (1-Blockage); Blockage }=\left(\text { Area }_{\text {Blocked }} / \text { Area }_{\text {Cross-section }}\right) \\ \phi & \text { Swirl angle } \\ \gamma & \text { Ratio of specific heats } \\ \bar{\omega} & \text { Relative average loss coefficient based upon inlet dynamic head } \\ \sigma & \text { Work done factor }\end{array}$

\section{Subscripts}

\section{Symbol Definition}

id Ideal

$\mathrm{r} \quad$ Radial direction

t Total

w Circumferential direction

$\mathrm{z} \quad$ Axial direction

1 Inlet to the compressor 
Exit to the compressor

\section{$\underline{\text { Superscripts }}$}

Relative to the blade 


\section{Appendix D - Control Volume Geometry}

Control volumes are needed through the bladed region to perform a control volume analysis to calculate turbomachinery source terms. The nomenclature used in this section is located in Section D.3.

\section{D.1 Control Volume Construction}

The SLCC control volumes are constructed with a Cartesian right-handed coordinate system consistent with the Cartesian control volumes of TEACC. The SLCC produces the same "axisymmetric flow with swirl" solution along each circumferential plane. It is through convenience only that a grid to calculate sources is constructed about the axial radial plane in the 12 o'clock position, as shown in Figure D.1, where the angle, $\theta$, is zero. The axial extents of the control volume trace out the lean associated with the leading and trailing edges of the blade defined by the axial stations used within the SLCC. This overall control volume is further broken into elementary control volumes with the streamline solution known in the center of the leading and trailing areas. Three planar views of the control volume, showing the symmetry in the circumferential direction with an angle of $\theta / 2$ on either side of the 12 o'clock position, are shown in Figure D.2. Each control volume axially extends the width of each blade, rendering a collection of radial control volumes, one above the other. The extreme inner and outer control volumes do not have a streamline solution moving through the center of the axial inlet and exit faces. A linear interpolation of adjacent streamlines is used for these 
extreme control volumes to construct flow-field information on the inlet and exit faces.

The areas and volumes of each hexahedral control volume, distributed through the blade, are calculated below with the circumferential segment of $\theta=0.5$ degrees (equivalent to 720 circumferential segments).

\section{D.2 Area and Volume Calculations}

Areas on the surface of each control volume must be constructed. Area is calculated the same way on each face by the cross product of diagonal position vectors completely defined by a four straight-sided, closed shape in a plane (Figure D.3a) as follows:

$$
A=\frac{1}{2}(C \times D)
$$

The position vectors are chosen to calculate an area which is always positive in the increasing index direction. Because of the unique symmetry involved in this problem, each face's area has only two non-zero components; these are listed in symbolic form below:

$$
\begin{aligned}
& \mathbf{A}_{\mathrm{I}}=\left(\mathrm{r}_{2}^{2}-\mathrm{r}_{1}^{2}\right) \cos \theta \sin \theta \mathbf{i}+\left(\mathrm{x}_{1}-\mathrm{x}_{2}\right)\left(\mathrm{r}_{2}+\mathrm{r}_{1}\right) \sin \theta \mathbf{j}+0 \mathbf{k} \\
& \mathbf{A}_{\mathrm{E}}=\left(\mathrm{r}_{3}^{2}-\mathrm{r}_{4}^{2}\right) \cos \theta \sin \theta \mathbf{i}+\left(\mathrm{x}_{4}-\mathrm{x}_{3}\right)\left(\mathrm{r}_{3}+\mathrm{r}_{4}\right) \sin \theta \mathbf{j}+0 \mathbf{k} \\
& \mathbf{A}_{\mathrm{T}}=\left(\mathrm{r}_{2}^{2}-\mathrm{r}_{3}^{2}\right) \cos \theta \sin \theta \mathbf{i}+\left(\mathrm{x}_{3}-\mathrm{x}_{2}\right)\left(\mathrm{r}_{3}+\mathrm{r}_{2}\right) \sin \theta \mathbf{j}+0 \mathbf{k} \\
& \mathbf{A}_{\mathrm{B}}=\left(\mathrm{r}_{1}^{2}-\mathrm{r}_{4}^{2}\right) \cos \theta \sin \theta \mathbf{i}+\left(\mathrm{x}_{4}-\mathrm{x}_{1}\right)\left(\mathrm{r}_{1}+\mathrm{r}_{4}\right) \sin \theta \mathbf{j}+0 \mathbf{k}
\end{aligned}
$$




$$
\begin{array}{r}
\mathbf{A}_{L}=0 \mathbf{i}+\frac{1}{2}\left[\left(x_{1}-x_{3}\right)\left(r_{4}-r_{2}\right)+\left(x_{2}-x_{4}\right)\left(r_{1}-r_{3}\right)\right] \sin \theta \mathbf{j}+ \\
\frac{1}{2}\left[\left(x_{3}-x_{1}\right)\left(r_{2}-r_{4}\right)+\left(x_{2}-x_{4}\right)\left(r_{1}-r_{3}\right)\right] \cos \theta \mathbf{k} \\
\mathbf{A}_{\mathbf{R}}=0 \mathbf{i}+\frac{1}{2}\left[\left(x_{3}-x_{1}\right)\left(r_{4}-r_{2}\right)+\left(x_{2}-x_{4}\right)\left(r_{3}-r_{1}\right)\right] \sin \theta \mathbf{j}+ \\
\frac{1}{2}\left[\left(x_{3}-x_{1}\right)\left(r_{2}-r_{4}\right)+\left(x_{2}-x_{4}\right)\left(r_{1}-r_{3}\right)\right] \cos \theta \mathbf{k}
\end{array}
$$

A quick check verified that the sum of the components of the area calculations made in each coordinate direction summed to zero.

When the areas are known, the volume can be calculated by dividing each sixsided hexahedral into six pyramids. Two opposing pyramids are shown in Figure D.3b. Each pyramid has one of the six faces as its base, and each of the six volumes is calculated from $1 / 3$ of the base area vector dotted with the distance from the face center to the volume center. Each of the opposing volumes can be calculated by:

$$
\text { Vol }=\frac{1}{3}\left(A \cdot \frac{\mathbf{d}_{\mathrm{IE}}}{2}\right)
$$

The total volume can then be constructed by adding all six volume pyramids together as follows:

$$
\mathrm{Vol}=\frac{1}{6}\left\{\begin{array}{l}
{\left[\left(A_{\mathrm{I}}-\mathbf{A}_{\mathrm{E}}\right) \cdot \mathbf{d}_{\mathrm{IE}}\right]+} \\
{\left[\left(\mathbf{A}_{\mathrm{B}}-\mathbf{A}_{\mathrm{Tt}}\right) \cdot \mathbf{d}_{\mathrm{BT}}\right]+} \\
{\left[\left(\mathbf{A}_{\mathrm{L}}-\mathbf{A}_{\mathrm{R}}\right) \cdot \mathbf{d}_{\mathrm{LR}}\right]}
\end{array}\right\}
$$

The above volume calculation is completely known, except for the vector distance from the center of opposing faces. First, the center of each face is found by averaging the 
position vectors of the four corners. The centers of opposing faces are differenced to describe a vector pointing in the direction of increasing index that passes through the center of the hexahedral. The final calculation of volume for the hexahedral is as follows:

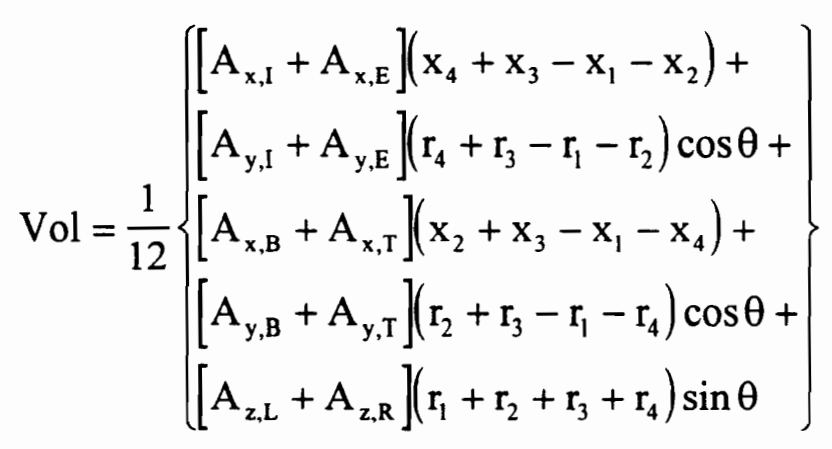

The areas and volumes of each hexahedral control volume distributed through the blade are calculated as described above and used with the control volume analysis to calculate turbomachinery source terms.

\section{D.3 Nomenclature for Appendix D}

\section{Symbol Definition}
$\mathbf{A}$
Area vector

A

Component of area in the $\mathrm{x}$-direction

C

Diagonal vector

d

Vector distance between two opposing volume centers

D

Diagonal vector

r

Radius

Vol

Volume

$\mathrm{x}, \mathrm{y}, \mathrm{z}$

Directions of Cartesian right-handed coordinate system 


\section{Symbol Definition}

$\theta$

Angle in the circumferential direction

\section{Subscripts}

I, E Inlet and exit control volume face

$\mathrm{L}, \mathrm{R} \quad$ Left and right control volume face

T, B Top and bottom control volume face

$\mathrm{x}, \mathrm{y}, \mathrm{z}$ Direction of Cartesian right handed coordinate system

1 Radius at location 1 of a control volume

2 Radius at location 2 of a control volume

3 Radius at location 3 of a control volume

4 Radius at location 4 of a control volume 


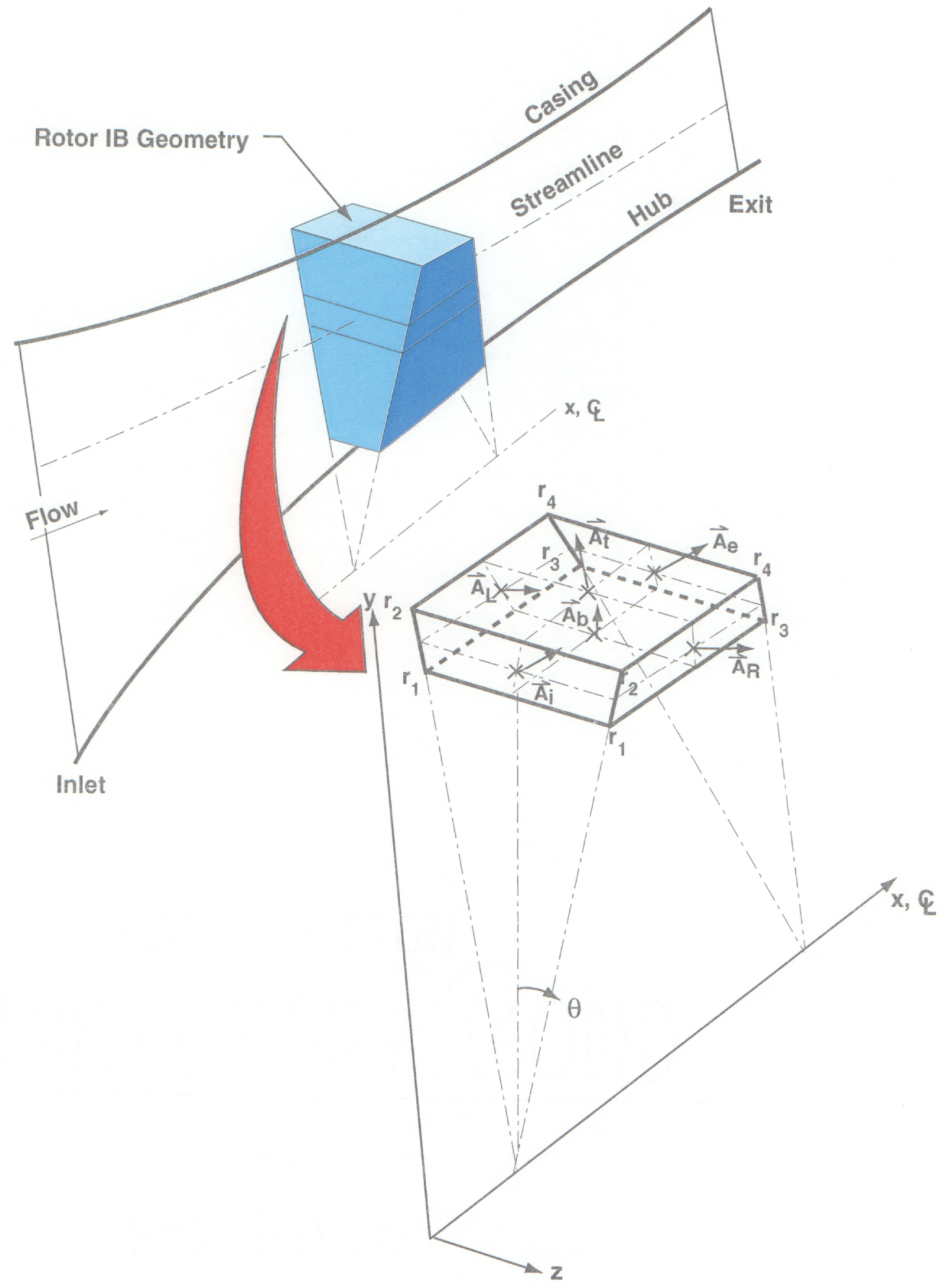

Figure D.1: Cartesian control volumes defined by Rotor 1B's geometry (circumferential segment, blade, and casing) and solution (streamlines). 


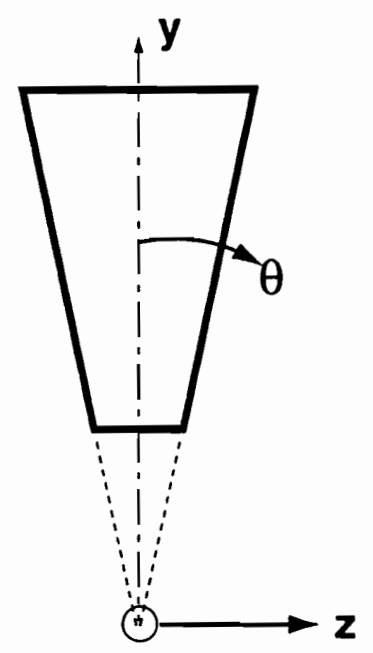

a. Axial slice

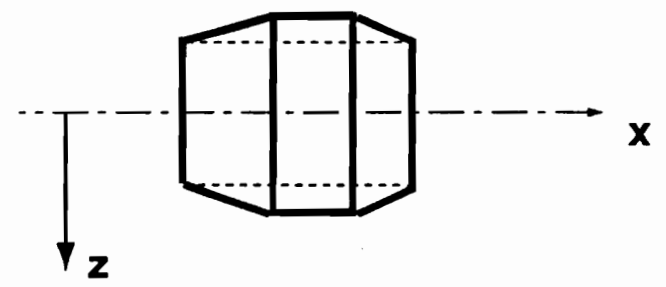

b. Radial slice

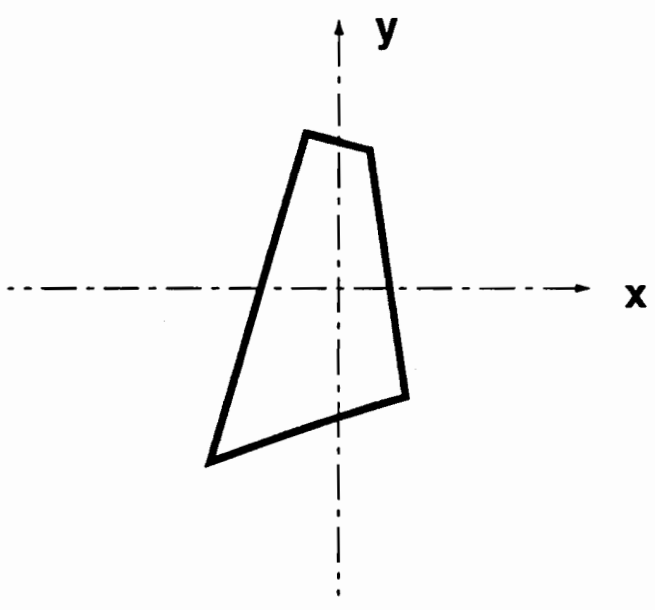

c. Circumferential slice

Figure D.2: Three planar views of the overall Cartesian control volume. 


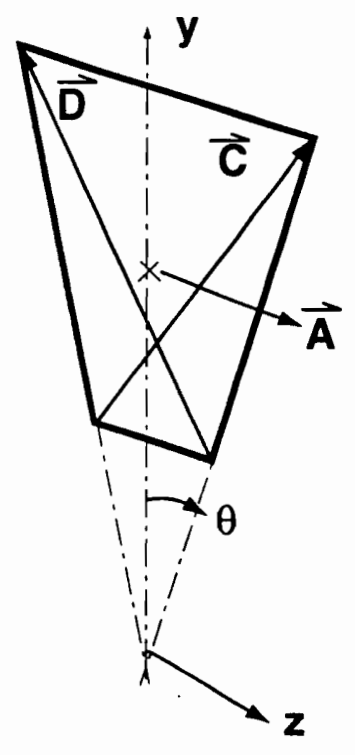

a. Area calculation

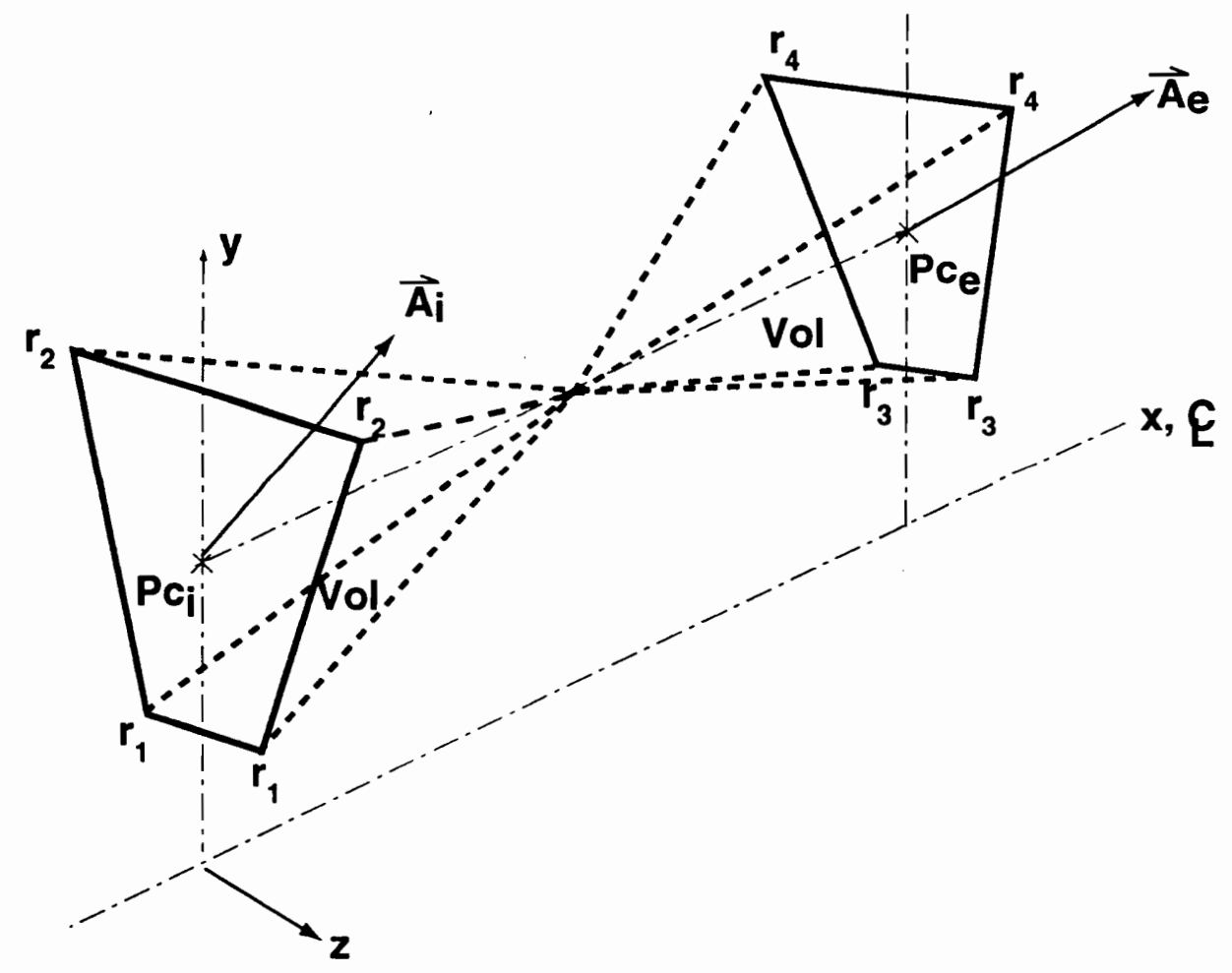

b. Volume calculation

Figure D.3: Technique to calculate area and volume. 


\section{Appendix E - Calibration of the SLCC for Rotor 1B}

The algorithm and assumptions behind the SLCC code simulation were developed in Appendix C. The current discussion will focus on calibrating and using the SLCC to model Rotor 1B along each pre-stall characteristic where data were supplied. This section is intended to present the results of the calibration of the SLCC using an overall geometry which extends far upstream and downstream of the compressor blades. A radial distribution of pre-stall steady-state characteristics is specified along each corrected speed line in the form of loss and exit relative flow angle to model the compressor performance. The overall geometry is specified, along with defining the inlet and exit of the bladed region as axial stations. With the SLCC constructed and calibrated, the results are compared to experimental data for overall performance. Since this is an axisymmetric simulation with swirl, a radial comparison can also be made for many flow-field quantities, both in the absolute and relative reference frame.

\section{E.1 SLCC Inputs}

The SLCC is a turbomachinery flow solver requiring a grid and boundary conditions. The overall geometry is specified in Figure E.1, with flow curvature assumed to be zero far upstream and far downstream of the compressor blades. The axial stations (axial grid lines) were vertical, except for those representing the compressor blades' inlet and exit. Additional axial stations could be modeled throughout the bladed region, but the bladed data available for compressor modeling was restricted to inlet, exit, and overall. Since no other restrictions were placed on the selection of the axial stations, they 
were selected based on the digital data provided, which has a stretching nature (axial grid lines becoming further apart away from the blade) toward the inlet and exit of the simulation. The inlet is defined approximately 30 inches in front of the blades, and the exit is defined approximately 12 inches behind the blades.

Total temperature and total pressure are inlet boundary conditions. The calibration assumed that inlet total temperature was $518.67 \mathrm{R}$ and the inlet total pressure was $14.696 \mathrm{psi}$. It was stated earlier that curvature as a function of radius is equal to zero at the upstream and downstream boundaries. This means that the streamlines are forced to be straight across the first two and last two axial stations, but they are free to be at an angle relative to the centerline. The overall mass flow rate must be specified and its value is maintained at every axial station as part of the SLCC convergence criteria. The user must also specify the number of streamlines used to model the flow field. Since data to model the compressor are available at only five radial locations, twice that number of streamlines smoothly captured the compressor behavior. Because a near mean-line streamline was desired for rough flow-field estimates, eleven streamlines were used for all subsequent calculations. No boundary layers or viscous effects were modeled along the hub and casing within the SLCC; however, these effects were present within the data used to model the compressor.

A technique was outlined in Appendix $\mathrm{C}$ for calculating absolute total temperature, absolute total pressure, and absolute swirl velocity from the empirical specification of relative total pressure loss coefficient and exit relative flow angle. The 
experimental data taken across Rotor $1 \mathrm{~B}$ gives a radial distribution of relative total pressure loss coefficient and exit relative flow angle. The data were taken at the two reference locations identified in Figure 5.1, where a data reduction package using streamlines was used to calculate the flow field on both sides of the compressor blades. As described earlier, loss and exit relative flow angle were taken at five different radii which were $10,30,50,70$, and $90 \%$ immersions of the local radial extents measured from the outer casing; they are presented in Figures E.2-E.6. This data are presented in five figures, one figure for each immersion location from the outer casing. Each figure shows loss verses corrected mass flow at a different immersion radius for each corrected speed at the top of the figure, and exit relative flow angle versus corrected mass flow for each speed at the bottom of the figure.

Some general comments are necessary about the loss and exit relative flow angle data. First, stall is located at the lower mass flow side and choke is located at the higher mass flow side. Second, the data are far from mapping out smooth curves of constant speed as the mass flow is increased. Unfortunately, no error bands were presented with the data to estimate its accuracy, so there is no unique way to know how to smooth the data. Therefore, the data were left exactly as taken, and no attempts were made to smooth out discrepancies. Third, the traditional loss buckets engineers are accustomed to seeing are usually loss versus incidence for a given corrected speed. It is evident from the figures that increasing mass flow behaves similarly to increasing incidence by forming the familiar loss bucket shape. Fourth, the overall shape of exit relative flow angle for a 
given speed tends to follow the shape of the loss for low speeds and for the middle half of the flow field for the higher speeds. For the two high speeds near the blade tip, exit relative flow angle increases while loss decreases. The loss behaves like it is far from choked since it continues to decrease with increasing corrected mass flow. The data from the higher speeds at the $90 \%$ immersion radius from the outer casing (near the hub) presented in Figure E.6 also had an unusual character. The loss behaves as though it is choked, but the exit relative flow angle decreases with increasing mass flow. Fifth, in general, the lowest part of the loss bucket at each radius increases with corrected speed, which is similar to the loss bucket lifting and becoming increasingly narrow with increased Mach number. Finally, exit relative flow angle decreases in magnitude at each immersion radius from the outer casing to the hub. The loss also decreases from the tip to the hub with an increase in the mean of the flow field due to the mid-span damper.

The data were implemented into the SLCC as a compressor map, where loss and exit relative flow angle were given as a function of radial location, corrected speed, and corrected mass flow. An option is available within the SLCC to perform a linear or spline curve fit interpolation between the data points. Both options were found to work well, since the data points were located close to each other. As pointed out earlier, overall mass flow must be specified and at convergence, maintained to a set tolerance for each axial station. The empirical compressor maps are based on corrected mass flow and corrected speed. The specified mass flow and speed from the empirical data are corrected as follows: 
corrected mass flow, $\dot{\mathrm{m}}_{\mathrm{c}}=\frac{\dot{\mathrm{m}} \sqrt{\frac{\mathrm{T}_{\mathrm{t}}}{\mathrm{T}_{\mathrm{ref}}}}}{\frac{\mathrm{P}_{\mathrm{t}}}{\mathrm{P}_{\mathrm{ref}}}}$

corrected rotor speed, $N_{c}=\frac{N}{\sqrt{\frac{T_{t}}{T_{r e f}}}}$.

A one-dimensional Mean-Line Code (MLC) was used to investigate the radial internal consistency for each data point on the overall compressor map. This technique was used to investigate the internal consistency of the radial distribution of the experimental data given. In general, the comparisons with experimental data produced results within $5 \%$ for all calculated flow-field variables at each point, except for two points on the $70 \%$ speed line. For these two points the MLC failed to match any of the experimental results. A more detailed investigation showed that these points were run significantly away from the $70 \%$ speed line, even though they were listed as $70 \%$ speed points. Therefore, these two data points were not included in the analysis.

The remaining input to the SLCC is the radial distribution of blade blockage in the flow field. Blockage is defined as a ratio of the blocked area to unblocked area of a compressor annulus and was provided with the SLCC as a means of accounting for the blockage due to the blades and boundary-layer growth. Radial blockage was used as a calibration quantity to bring the total temperature ratio and the total pressure ratio in line with the presented experimental data. The magnitudes of blockage are presented like a compressor map in Figures E.7 - E.11. Blockage is specified as a function of inlet radius, 
corrected speed, and corrected mass flow. It was found for all flow points that the better the radial distribution of temperature ratio and pressure ratio matched the experimental data by changing blockage, the closer the radial distribution of all other flow variables matched the experimental data. As expected, the largest physical values in blockage are located near the tip.

A radial distribution of blockage was used as a calibration tool to correct the total pressure ratio and the total temperature ratio, primarily at the rotor tip. Changing the blockage has the same effect as varying the loss or deviation. Since a radial distribution of loss and deviation was specified from experimental data, the unknown blockage was used as a fine tuning mechanism. Blockage was allowed to be both positive and negative, of which negative is nonphysical.

\section{E.2 Overall Comparison of SLCC with Experimental Data}

The SLCC is a fundamental tool combining first principles and empirical relations for calculating the flow field through the compressor. A comparison of the total pressure ratio and the efficiency with experimental data is presented in Figure E.12 as a function of corrected mass flow rate for the $50,70,90$, and $100 \%$ design corrected speeds. The data are listed as symbols and the SLCC results are presented as lines. The pressure ratio and the efficiency are in excellent agreement with the character and magnitude of each point for each speed. This good agreement means that from an overall perspective, the calibration is sophisticated enough to cause the overall pressure ratio and efficiency to match the experimental data. It is noted in Figure E.12a that the compressor stalls at the 
left end of each corrected speed line. For the two higher corrected speed lines, the characteristics choke on the right where the mass flow rate no longer increases as the pressure ratio continues to decrease. The efficiency in Figure E.12b is particularly interesting. Efficiency does not vary smoothly along a constant speed, but the SLCC still does an excellent job of matching the overall data. The overall character of efficiency at a given speed behaves as expected, with the highest values located close to the middle of the corrected mass flow range and efficiency decreasing near stall and choke. Matching overall pressure ratio and overall efficiency is significant, but matching the radial distribution of experimental data is also important. A high rotor speed point has been selected near the throttle line for this detailed investigation. All points in the flow field compare similarly to this point.

\section{E.3 Radial Comparison of SLCC with Experimental Data}

A comparison of the SLCC with experimental data is done only at a single operating point because this is representative of all operating points. Several variables are selected and compared with experimental data to evaluate the calibration process. The maximum percent difference with experimental data for each variable is presented in Table E.1, along with the figure number of the comparison with data.

The total pressure ratio and total temperature ratio along the radial direction are compared with experimental data in Figures E.13 and E.14. A consistent trend for most data points for Rotor $1 \mathrm{~B}$ is that total pressure ratio and total temperature ratio increase with increasing radius. Since the mass flow rate also increases radially, this means that 
the compressor did not produce uniform work, but rather the amount of work increased from hub to tip. The maximum percent difference with experimental data for total pressure ratio and for total temperature ratio is less than 0.1-percent, providing excellent agreement with experimental data. This is to be expected, since the blockage was specified to force radial agreement during the calibration process. Since the flow field specifies uniform total pressure and total temperature at the inlet, the exit total pressure and exit total temperature displayed in Figures E.15 and E.16 have the expected same shape as the respective previously presented total ratios. Exit total pressure and exit total temperature compare within 0.1 percent of experimental data. These variables are in excellent agreement with experimental data.

Exit static pressure and exit static temperature are also compared to experimental data in Figures E.17 and E.18, respectively. Static pressure and static temperature generally follow the shape of their respective totals, and there was no exception in this case. The tip of the compressor is highly backpressured. Exit static pressure and exit static temperature compare within 2-percent of experimental data. Both compare well with data in magnitude and shape. Two other highly related variables are exit absolute velocity and exit absolute Mach number. These are compared to experimental data in Figures E.19 and E.20. Close agreement of these shapes is expected, and it can be seen that they are also in excellent agreement with data. Both exit absolute velocity and exit Mach number can be seen from the figures to have less than 1.0-percent difference with 
experimental data. For this high rotor speed point, the absolute exit Mach number is far from transonic at just over 0.6 .

The exit absolute circumferential velocity presented in Figure E.21 has the greatest difference with experimental data over all the other variables in the flow field, as can be seen in Table E.1. This difference takes place near the tip; however, the overall shape compares well with experimental data. This is a very important variable, because the product of absolute circumferential velocity and exit wheel speed with zero inlet circumferential velocity is the essence of the work produced by the compressor calculated from the Euler turbine equation. Since wheel speed is increasing radially, this confirms the previous statement that work is increasing with increasing radius. Static density has already been used in the calculation of the speed of sound for exit Mach number and is presented in Figure E.22. It also compares well with experimental data.

Comparisons relative to the blade were also made with experimental data. The magnitude of inlet and exit relative velocity were compared with data in Figures E.23 and E.24, respectively. They each have good agreement with experimental data. As expected from velocity diagrams where the absolute inlet velocity increases through the compressor, the relative velocity decreases from the inlet to the exit. The same trend is viewed by looking at the relative inlet Mach number (Figure E.25) and the relative exit Mach number (Figure E.26). Clearly, this is a highly transonic rotor with an inlet relative Mach number of 1.40 at the tip. The exit relative Mach number has a strong radial distribution ranging from 0.5 at the hub to 0.9 at the tip. Both variables have the same 
overall shape, with exit relative Mach number deviating the most from experimental data, within 3.0-percent. Although there is zero absolute circumferential velocity entering the compressor, the rotating blades produced strong relative inlet and exit circumferential velocity, as seen in Figures E.27 and E.28, respectively. Also, from velocity triangles it is expected that the absolute circumferential velocity will strongly increase through the rotor and the relative circumferential velocity will decrease through the blades. The relative circumferential velocity variables compare well with experimental data in both magnitude and shape. With the completion of the calibration process, the SLCC was used for the calculation of source terms in TEACC. 
Table E.1: Calibrated streamline curvature code (SLCC) compared with experimental data.

\begin{tabular}{|l|c|c|}
\hline \multicolumn{1}{|c|}{ Variable } & Figure Number & Max. Percent Difference \\
\hline Total Pressure Ratio & E.13 & -0.0364 \\
\hline Total Temperature Ratio & E.14 & 0.0862 \\
\hline Exit Total Pressure & E.15 & -0.0368 \\
\hline Exit Total Temperature & E.16 & 0.0852 \\
\hline Exit Static Pressure & E.17 & -0.1498 \\
\hline Exit Static Temperature & E.18 & 1.8666 \\
\hline Exit Absolute Velocity & E.19 & 0.2381 \\
\hline Exit Mach Number & E.20 & 0.8528 \\
\hline Exit Circumferential Velocity & E.21 & 4.3591 \\
\hline Exit Static Density & E.22 & -0.1597 \\
\hline Inlet Relative Velocity & E.23 & -1.0838 \\
\hline Exit Relative Velocity & E.24 & -2.9337 \\
\hline Inlet Relative Mach number & E.25 & -1.3071 \\
\hline Exit Relative Mach number & E.26 & -2.7443 \\
\hline Exit Relative Circumferential Velocity & E.27 & -0.0621 \\
\hline Exit Relative Circumferential Velocity & E.28 & -2.9539 \\
\hline
\end{tabular}




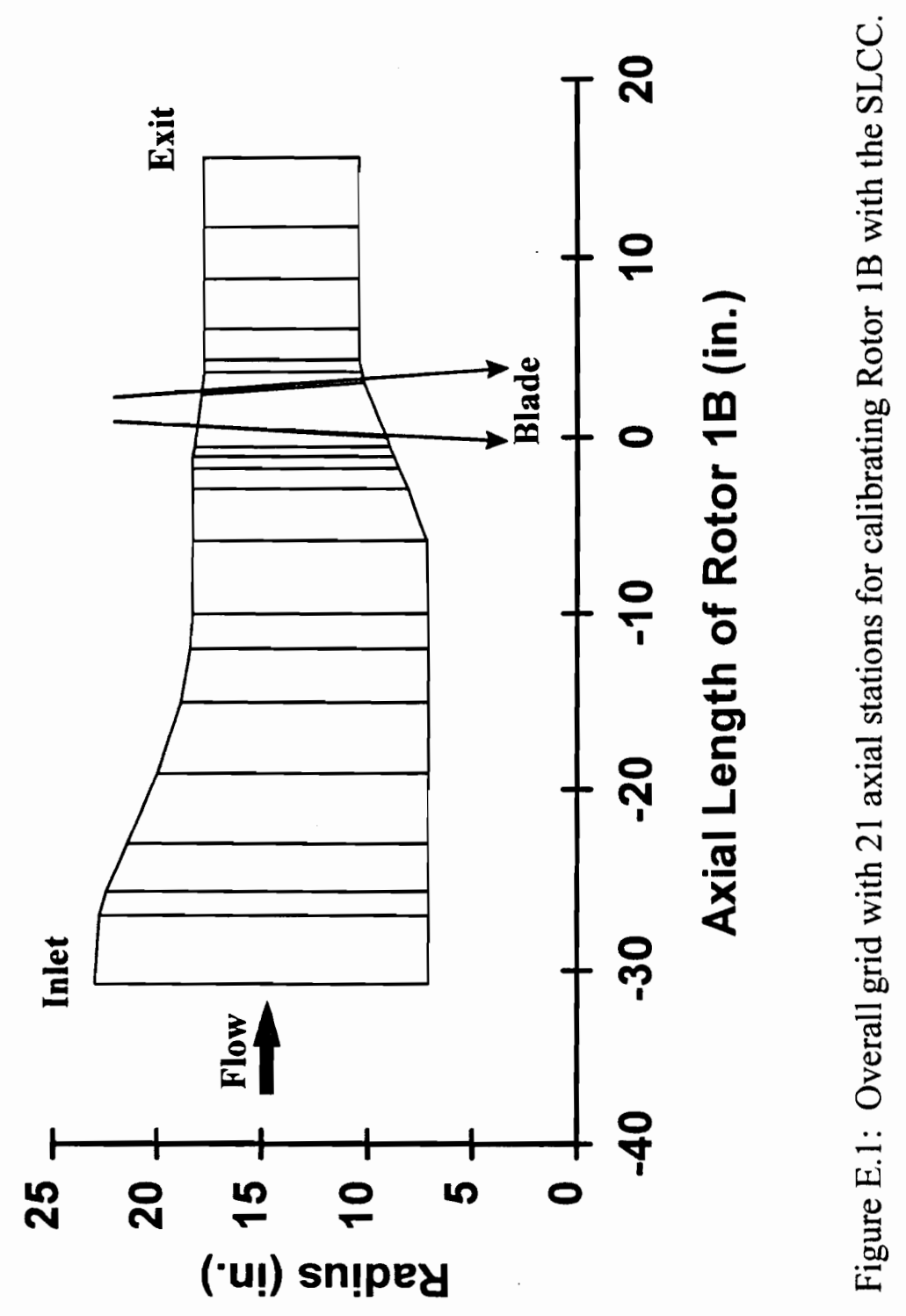



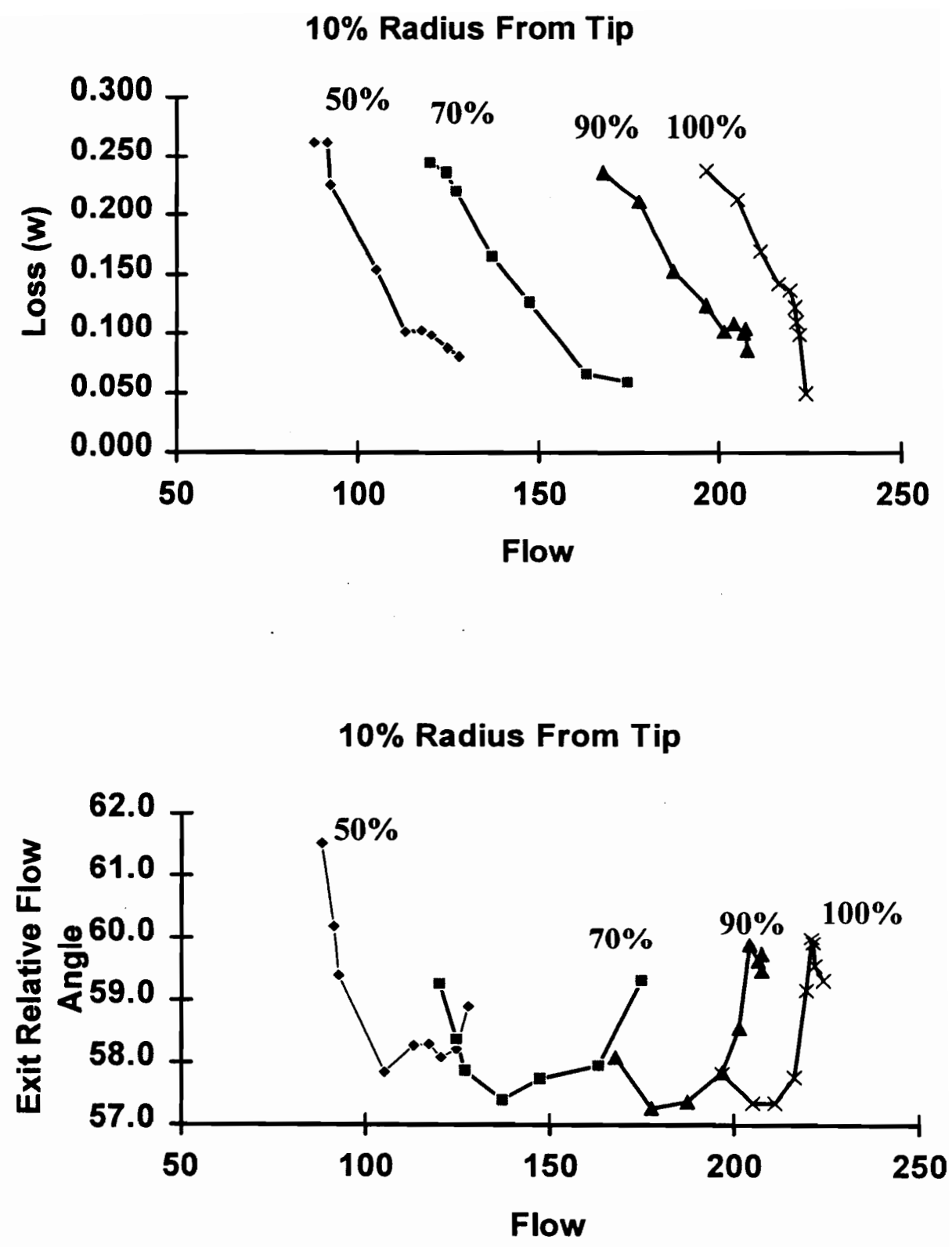

Figure E.2: Loss and exit relative flow angle at 10-percent radial immersion from the outer casing tip. 

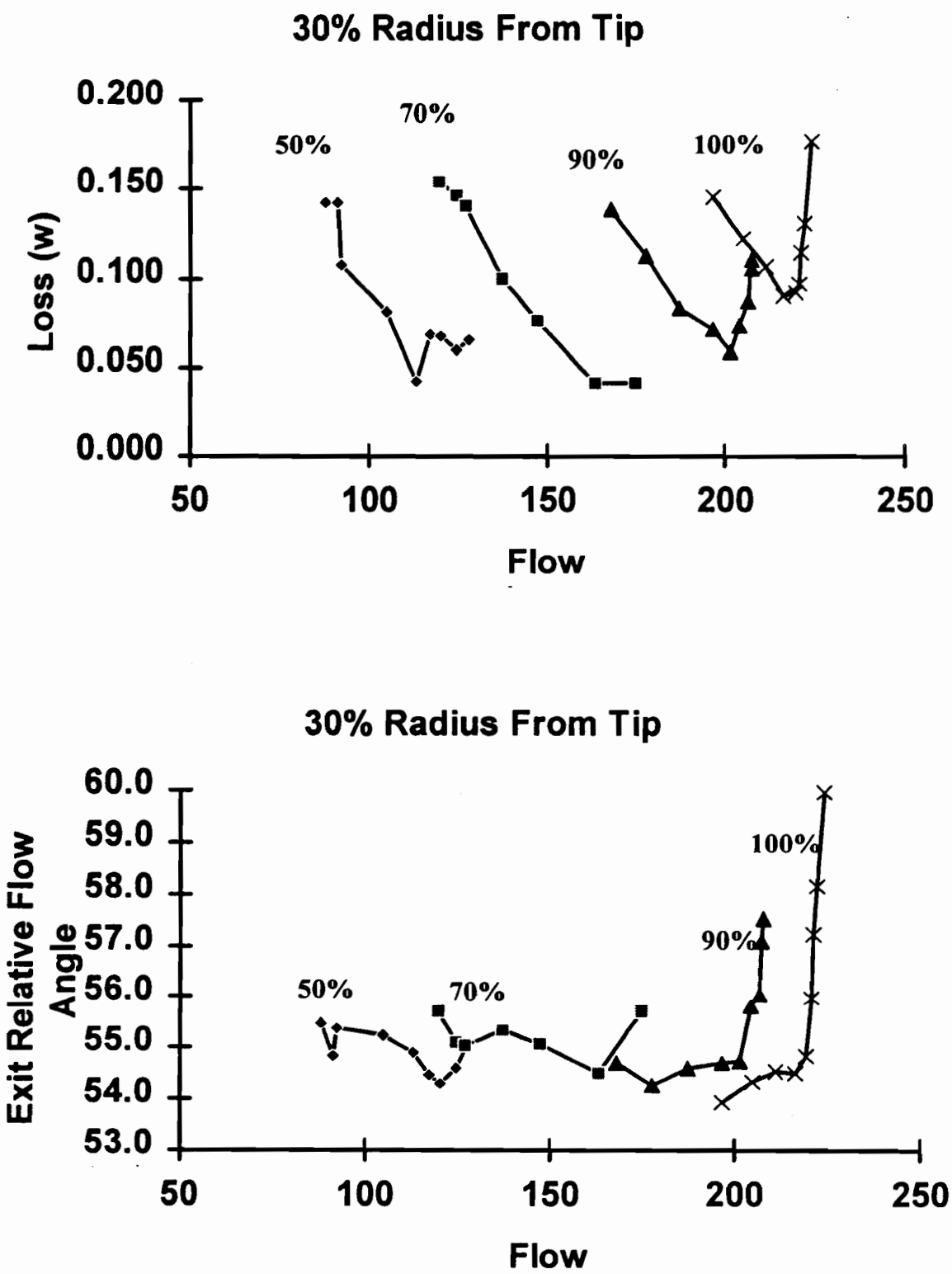

Figure E.3: Loss and exit relative flow angle at 30-percent radial immersion from the outer casing tip. 
$50 \%$ Radius From Tip
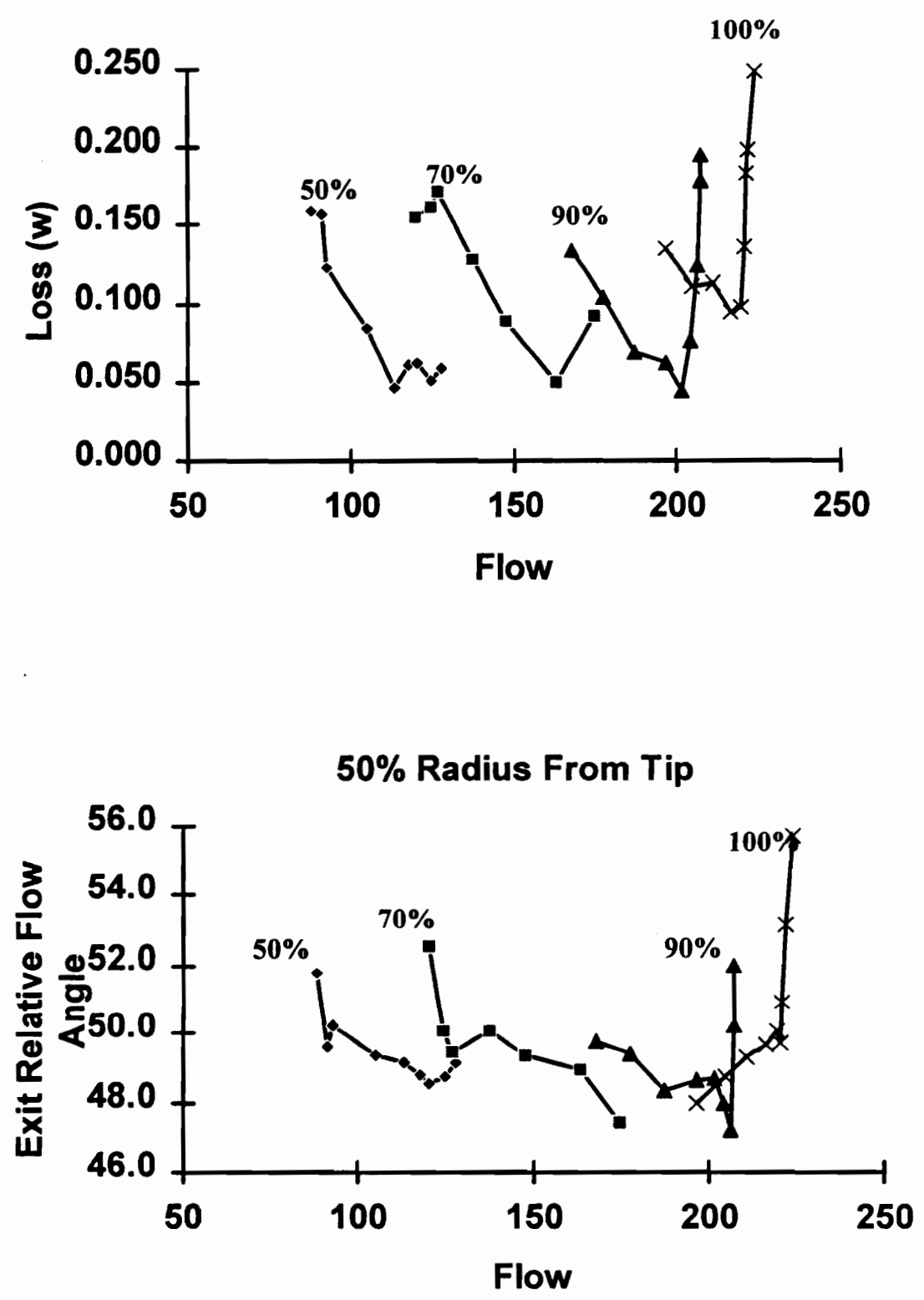

Figure E.4: Loss and exit relative flow angle at 50-percent radial immersion from the outer casing tip. 
70\% Radius From Tip

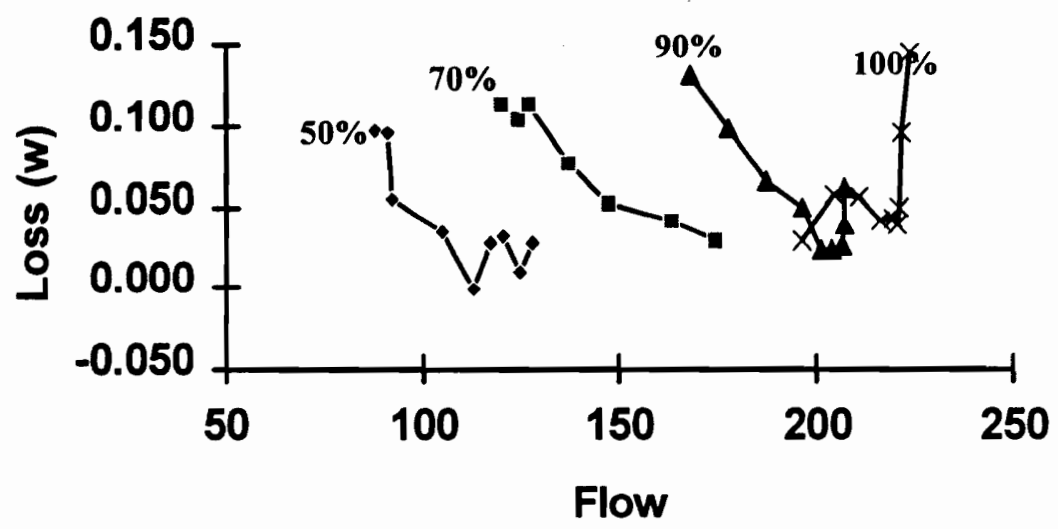

70\% Radius From Tip

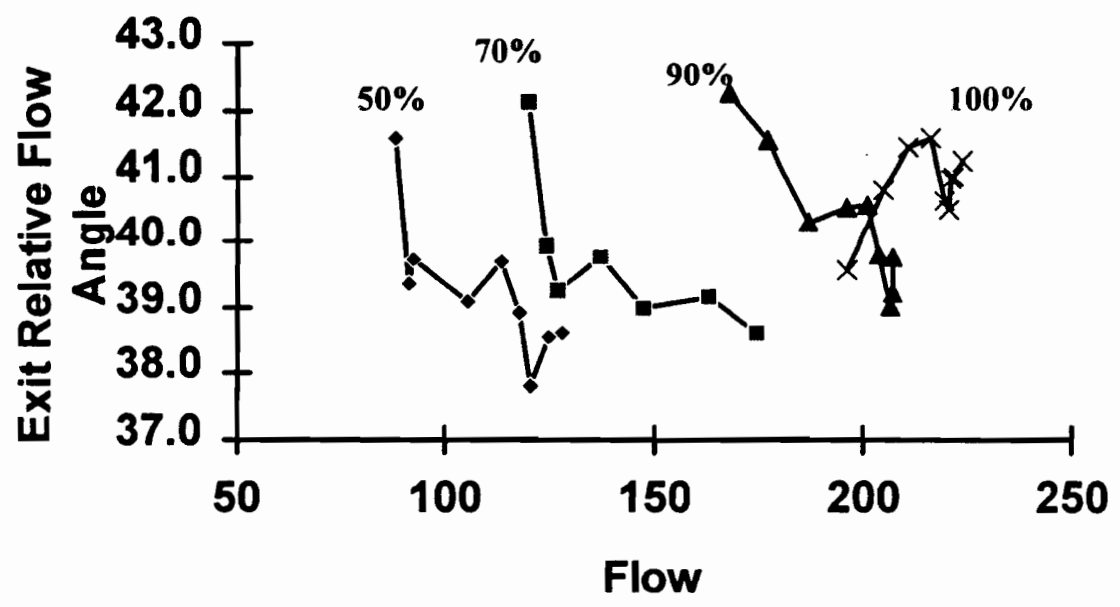

Figure E.5: Loss and exit relative flow angle at 70-percent radial immersion from the outer casing tip. 


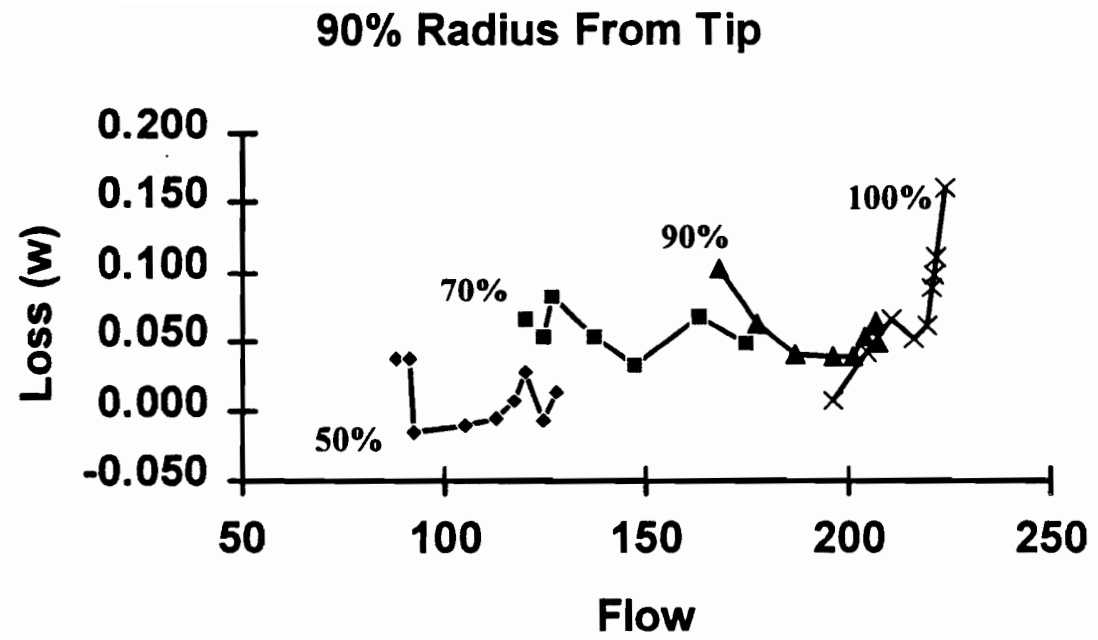

90\% Radius From Tip

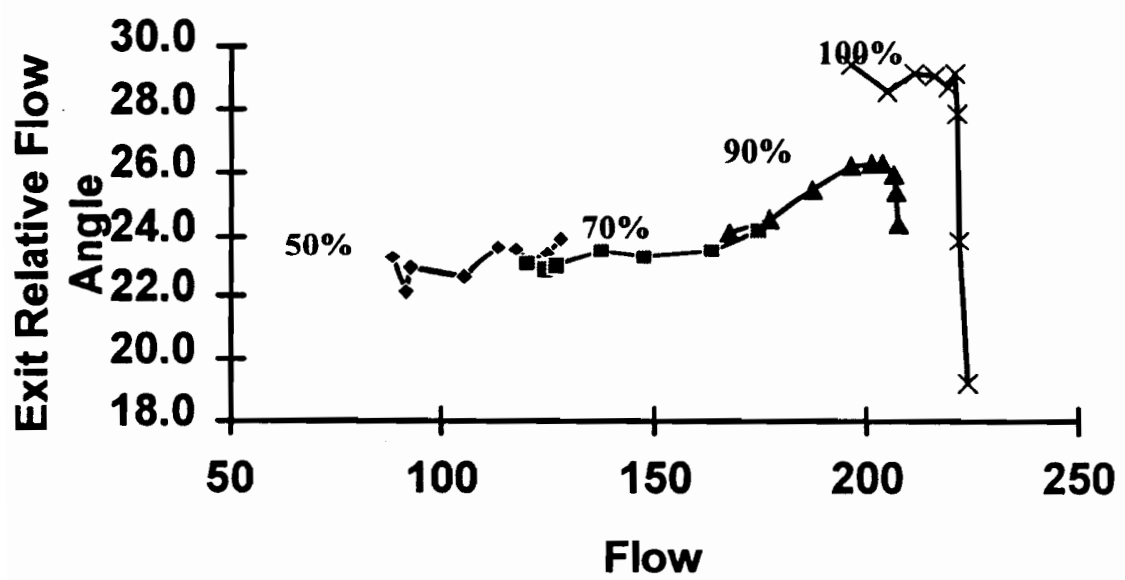

Figure E.6: Loss and exit relative flow angle at 90-percent radial immersion from the outer casing tip. 


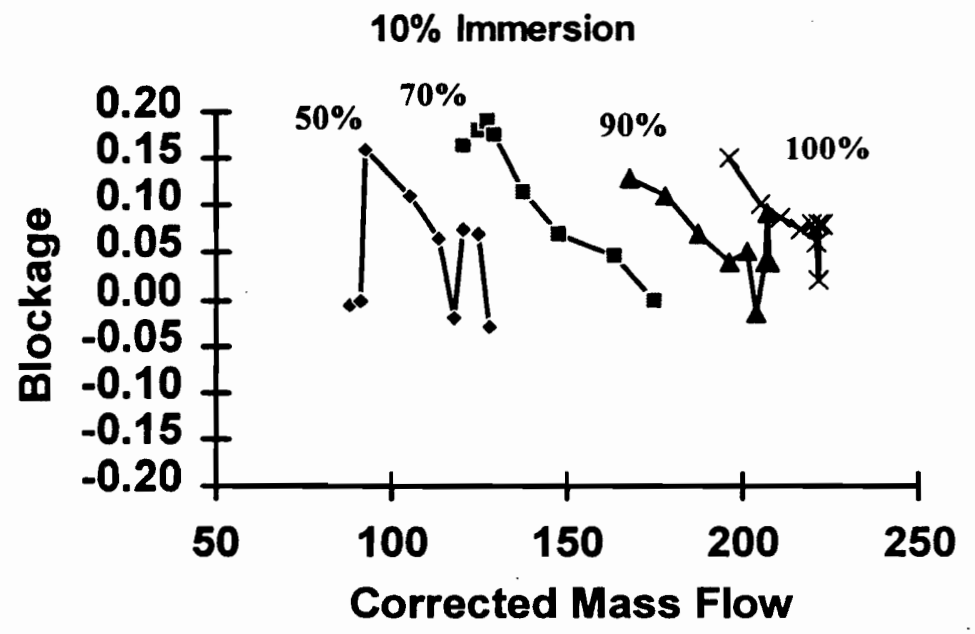

Figure E.7: Blockage at 10-percent radial immersion from the outer casing tip.

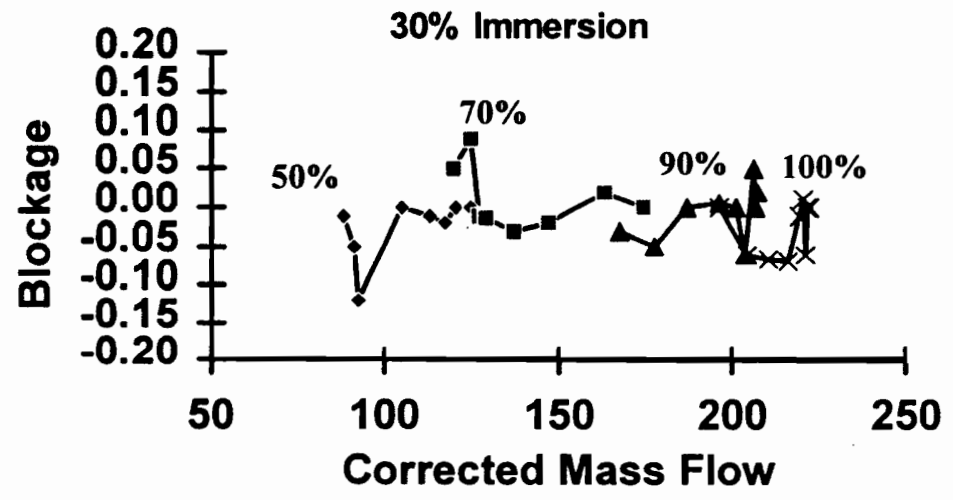

Figure E.8: Blockage at 30-percent radial immersion from the outer casing tip 


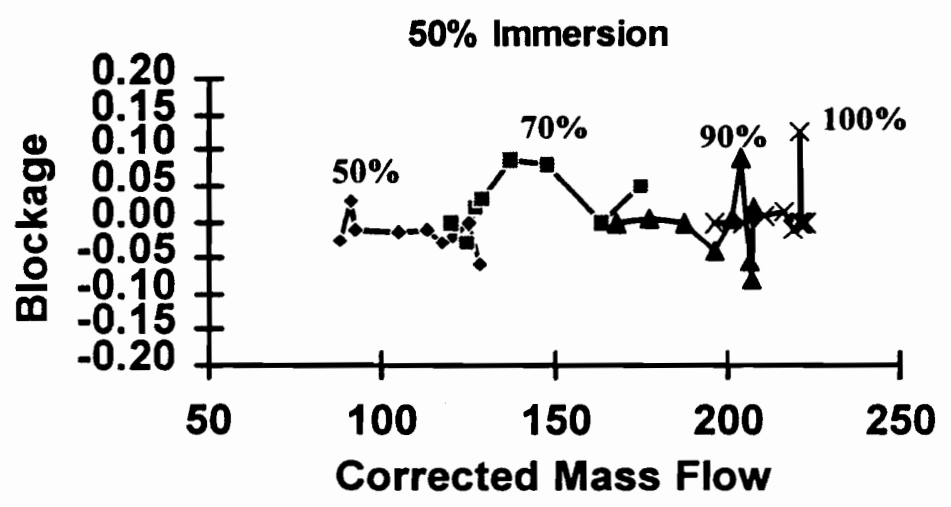

Figure E.9: Blockage at 50-percent radial immersion from the outer casing tip.

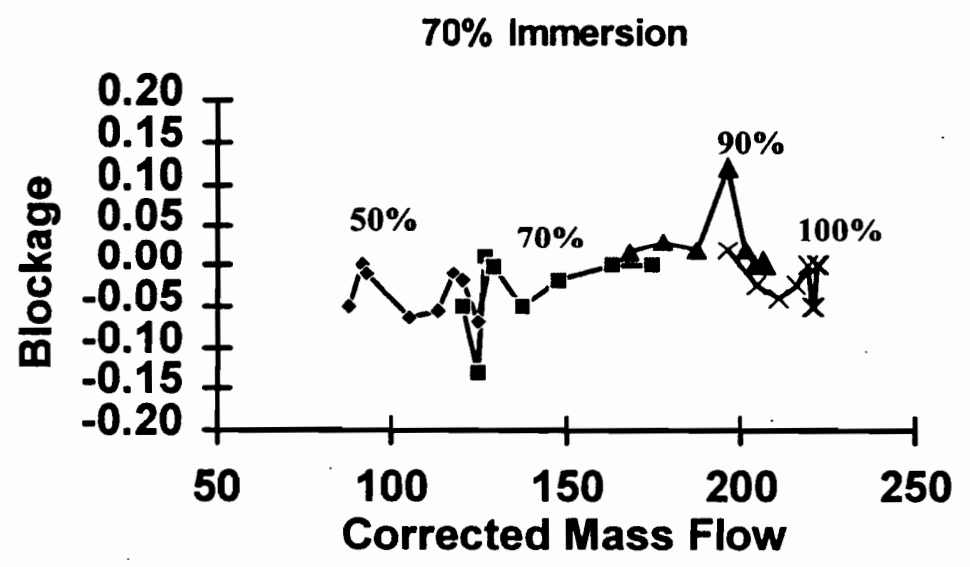

Figure E.10: Blockage at 70-percent radial immersion from the outer casing tip. 


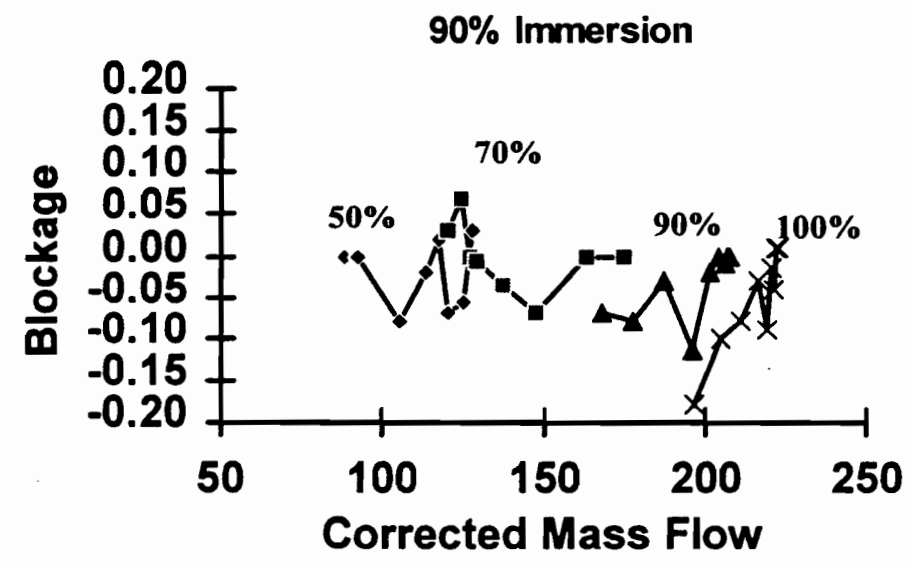

Figure E.11: Blockage at 90-percent radial immersion from the outer casing tip. 
Pressure Ratio From SLCC Compared to Data

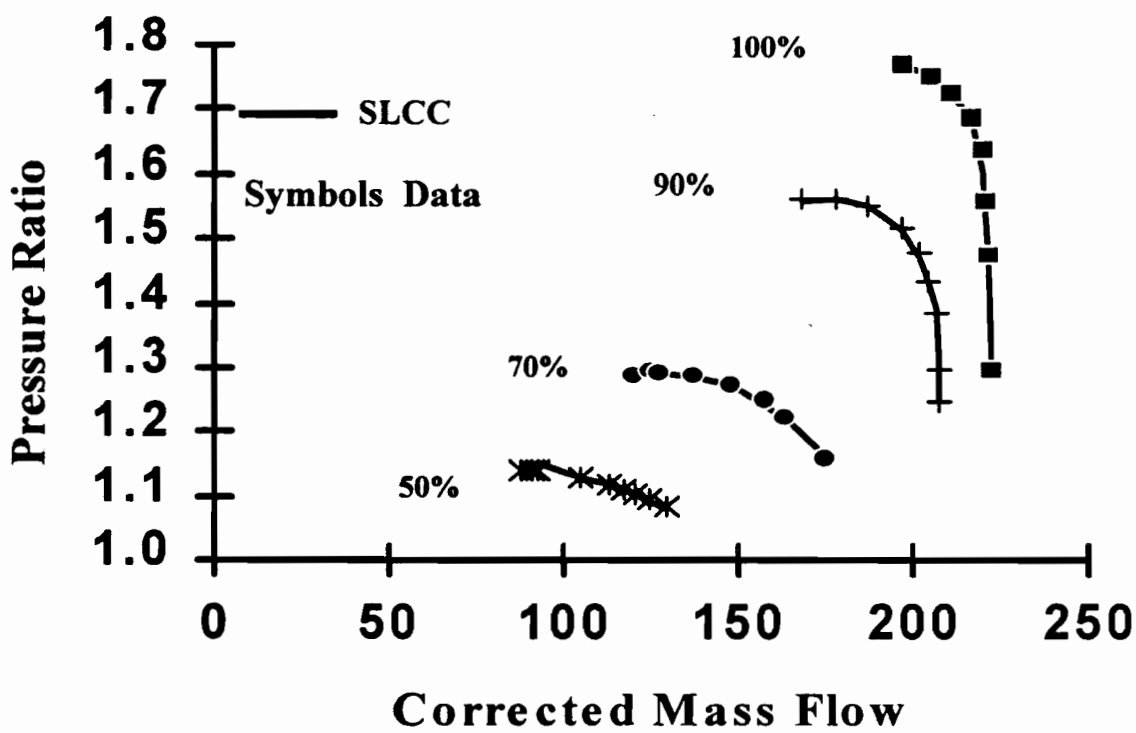

Efficiency From SLCC Compared to Data

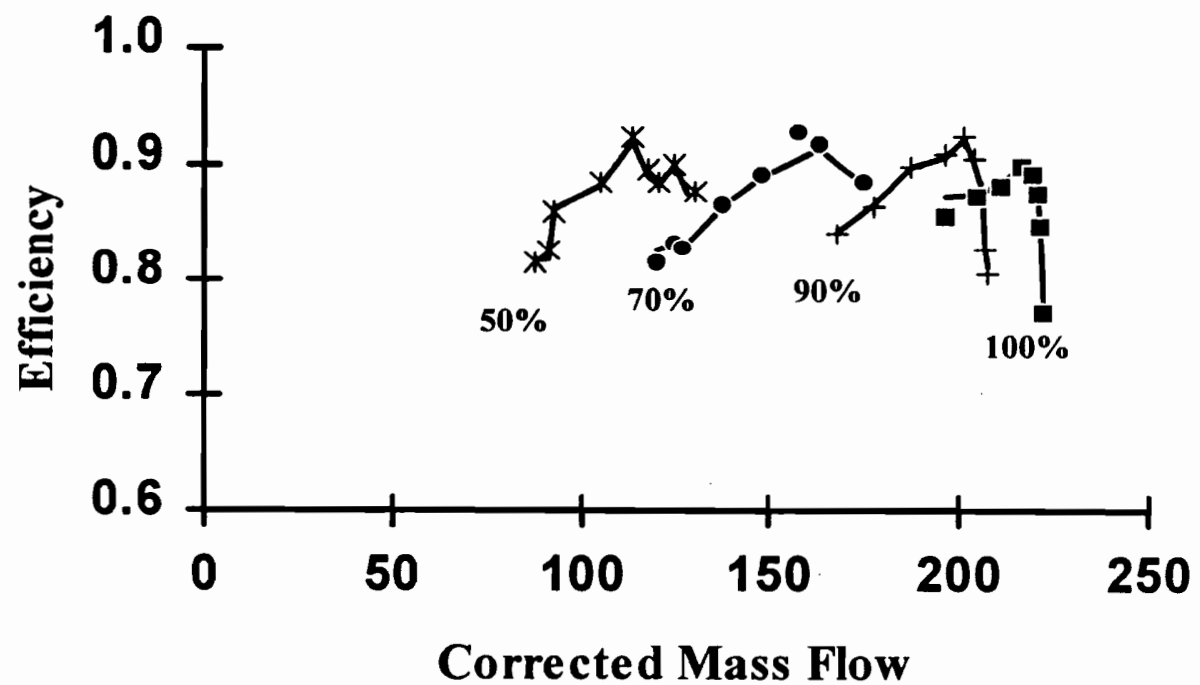

Figure E.12: Overall comparison of the SLCC calibration with experimental data in total pressure ratio and efficiency for $50,70,90$, and $100 \%$ speed. 
$\checkmark$ Data - SLCC

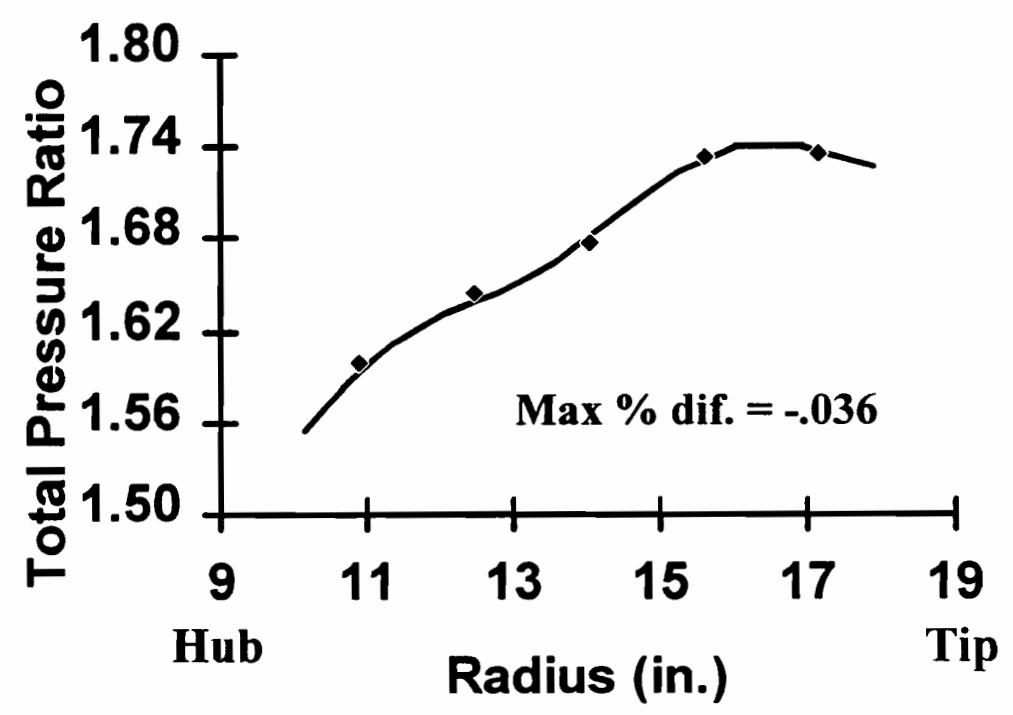

Figure E.13: Calibration, total pressure ratio on the $100 \%$ corrected speed line near the design throttle line.

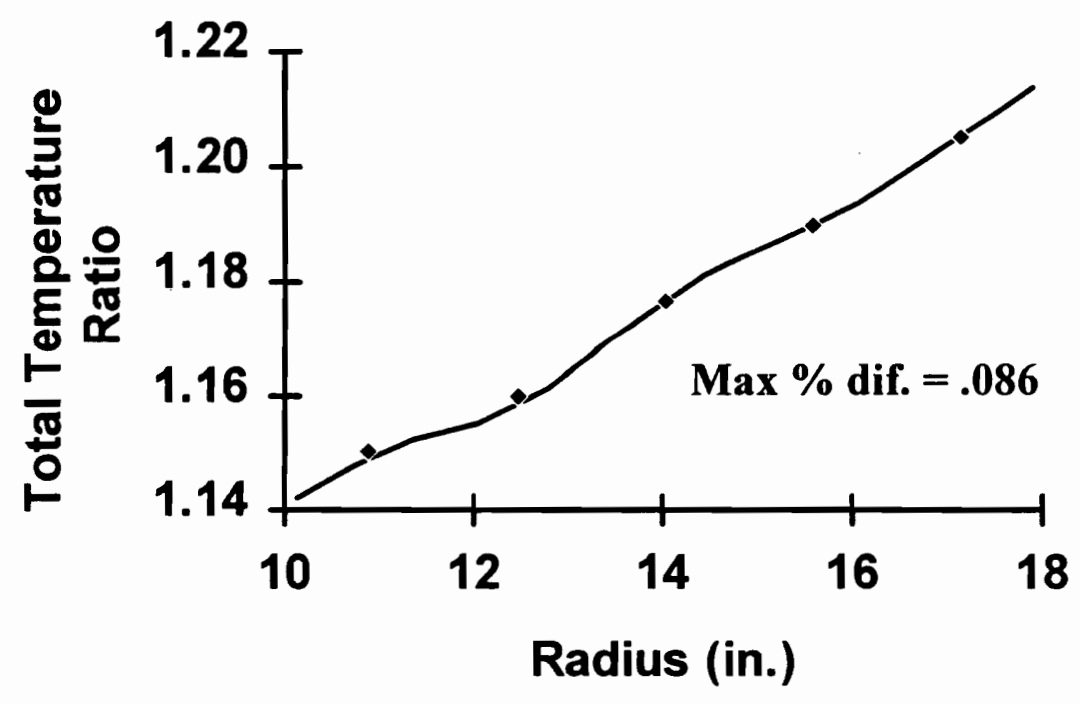

Figure E.14: Calibration, total temperature ratio on the $100 \%$ corrected speed line near the design throttle line. 


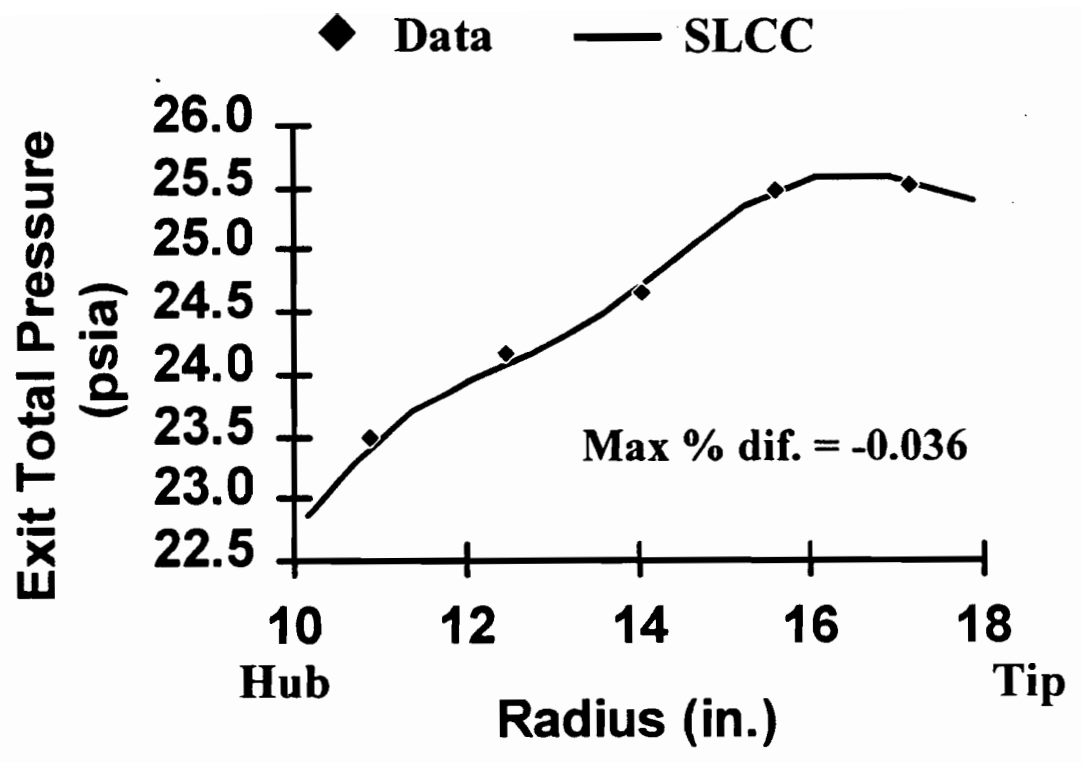

Figure E.15: Calibration, exit total pressure ratio on the $100 \%$ corrected speed line near the design throttle line.

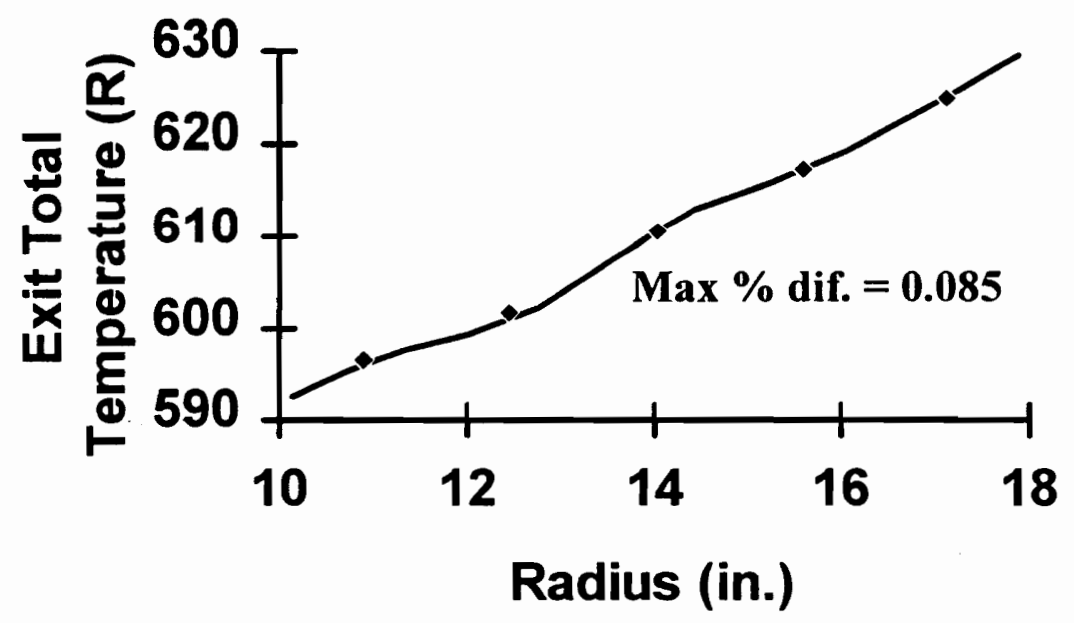

Figure E.16: Calibration, exit total temperature ratio on the $100 \%$ corrected speed line near the design throttle line. 
- Data $\longrightarrow$ SLCC

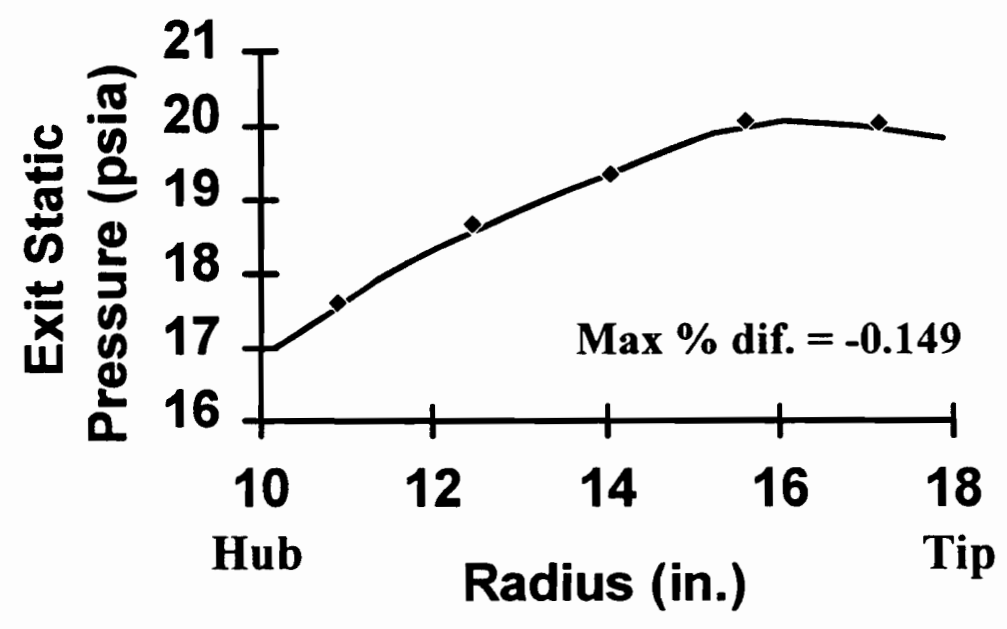

Figure E.17: Calibration, exit static pressure ratio on the $100 \%$ corrected speed line near the design throttle line.

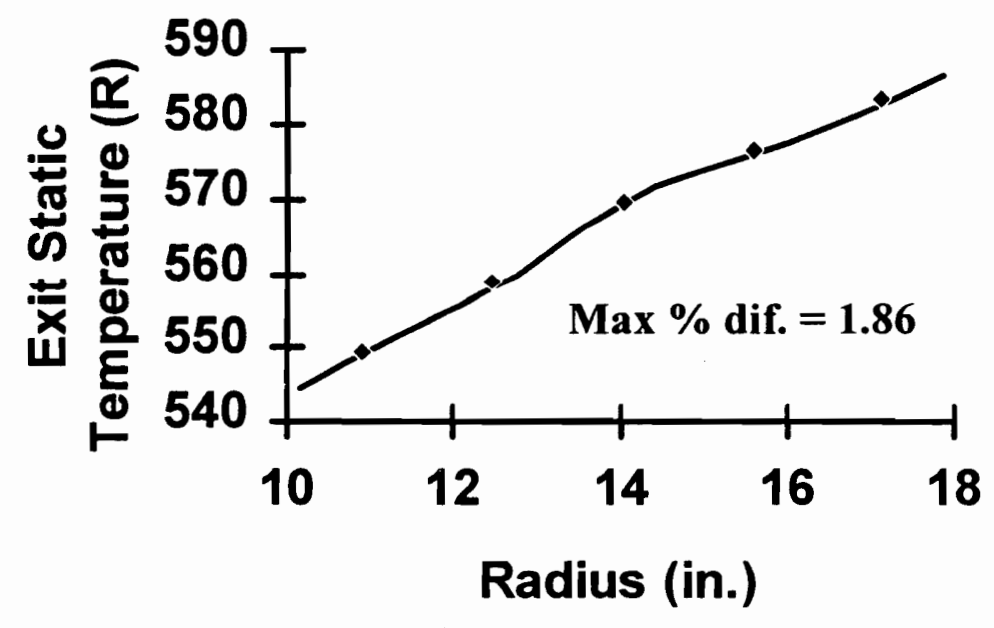

Figure E.18: Calibration, exit static temperature ratio on the $100 \%$ corrected speed line near the design throttle line. 


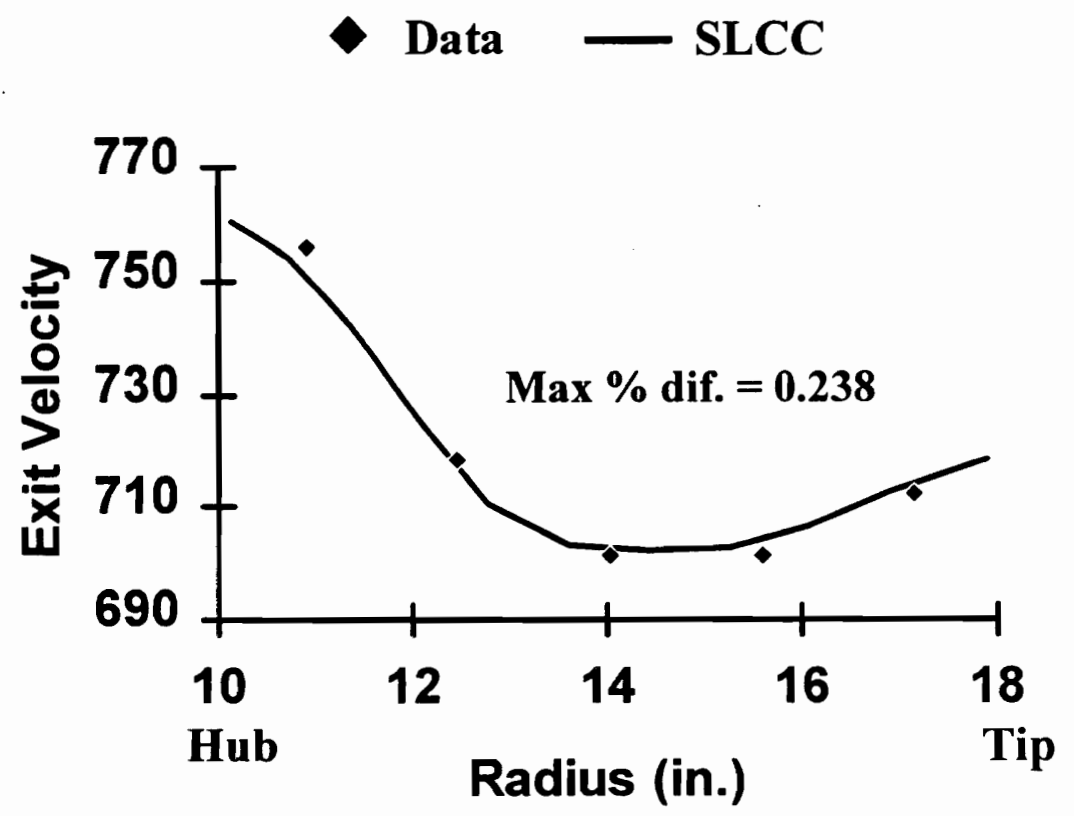

Figure E.19: Calibration, exit velocity on the $100 \%$ corrected speed line near the design throttle line.

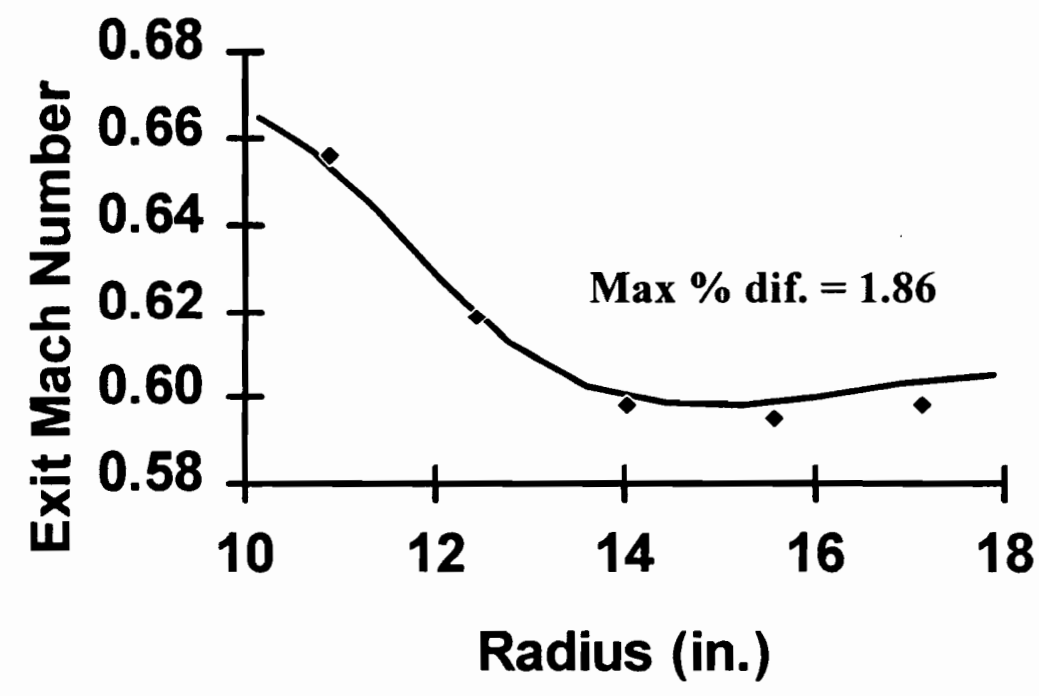

Figure E.20: Calibration, exit Mach number on the 100\% corrected speed line near the design throttle line. 


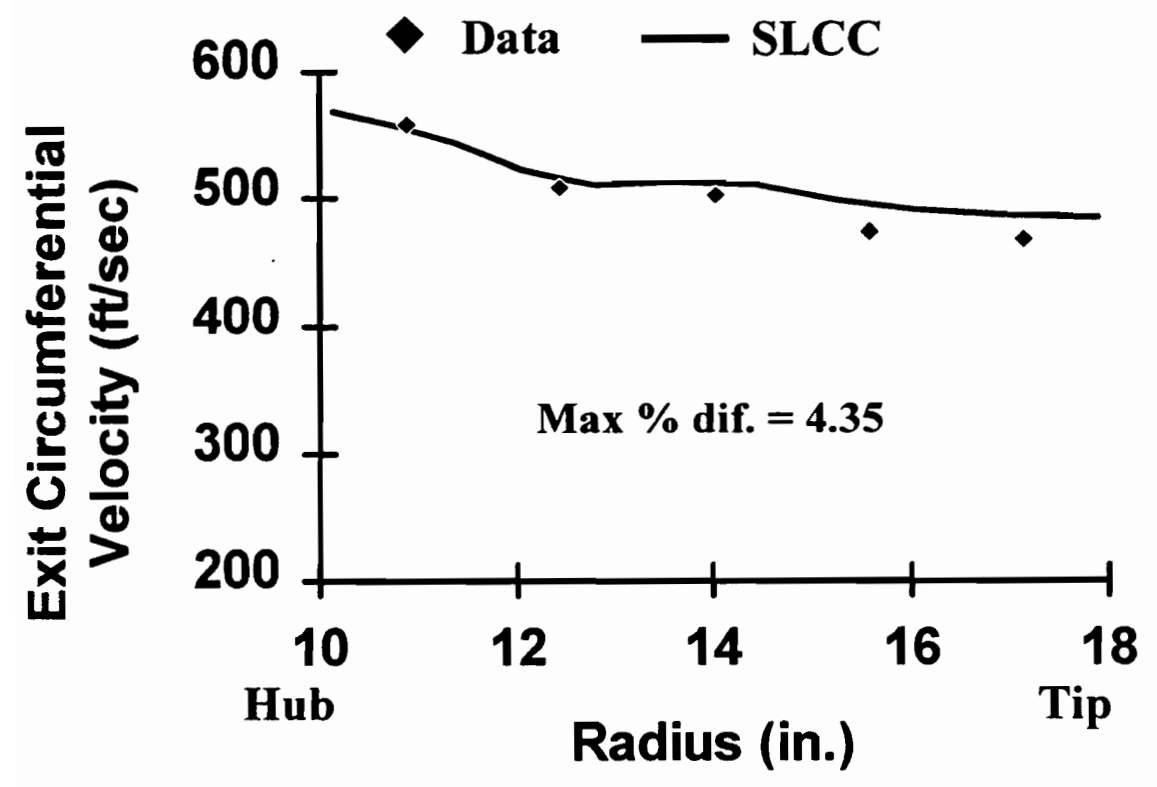

Figure E.21: Calibration, exit circumferential velocity on the $100 \%$ corrected speed line near the design throttle line.

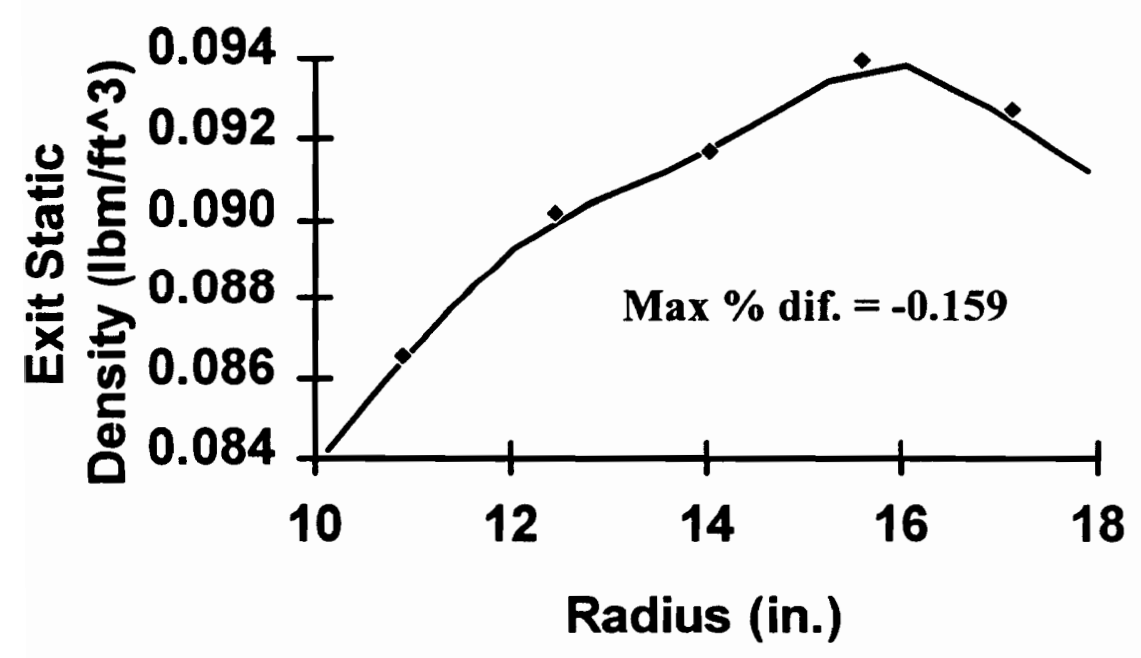

Figure E.22: Calibration, exit static density on the $100 \%$ corrected speed line near the design throttle line. 
$\checkmark$ Data - SLCC

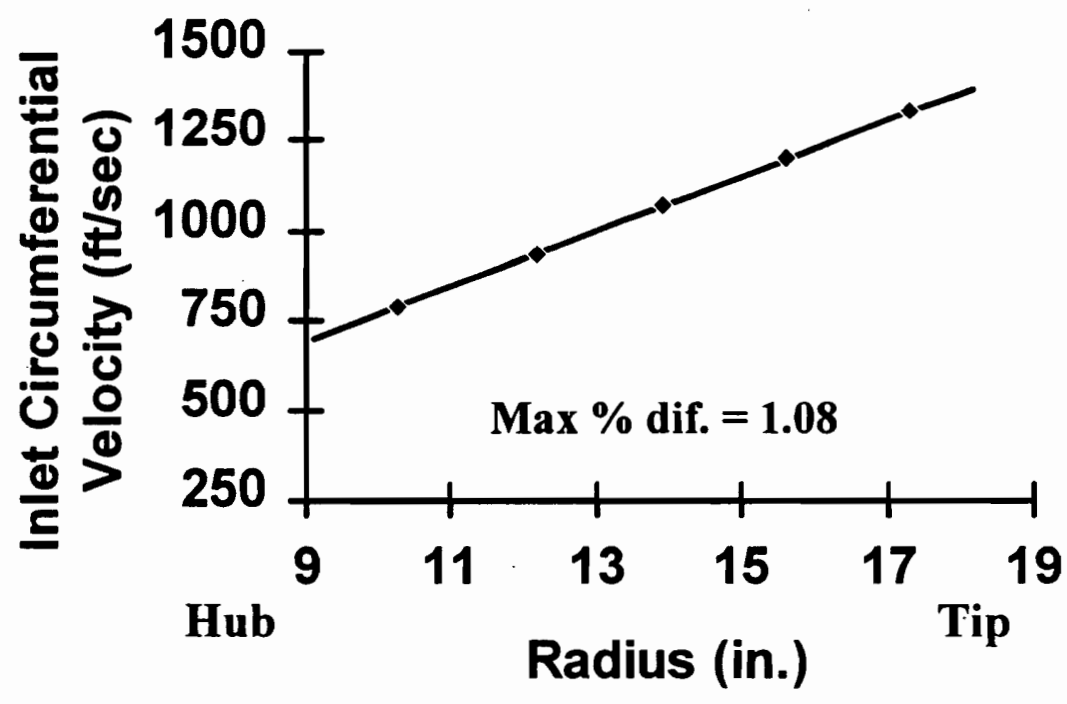

Figure E.23: Calibration, inlet relative velocity on the $100 \%$ corrected speed line near the design throttle line.

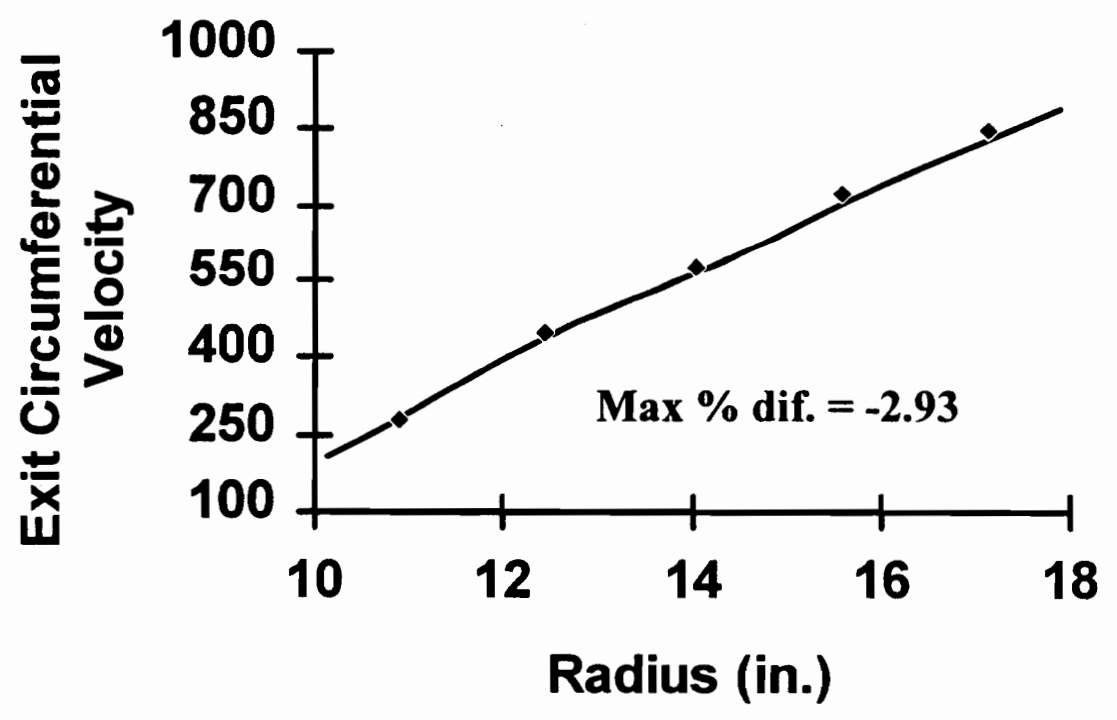

Figure E.24: Calibration, exit relative velocity on the $100 \%$ corrected speed line near the design throttle line. 
$\checkmark$ Data $\longrightarrow$ SLCC

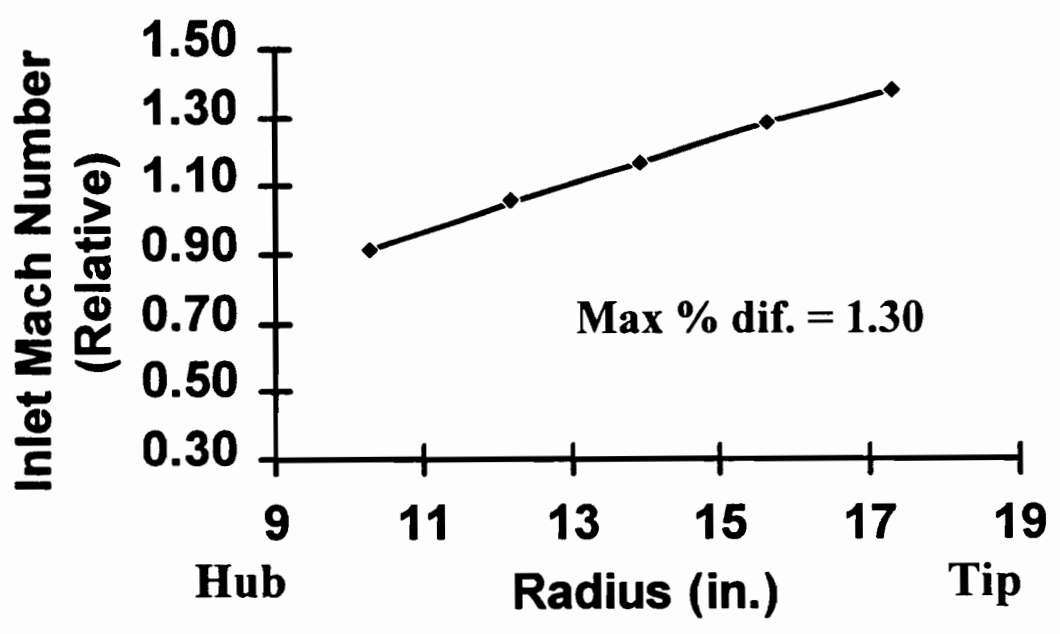

Figure E.25: Calibration, inlet relative Mach number on the 100\% corrected speed line near the design throttle line.

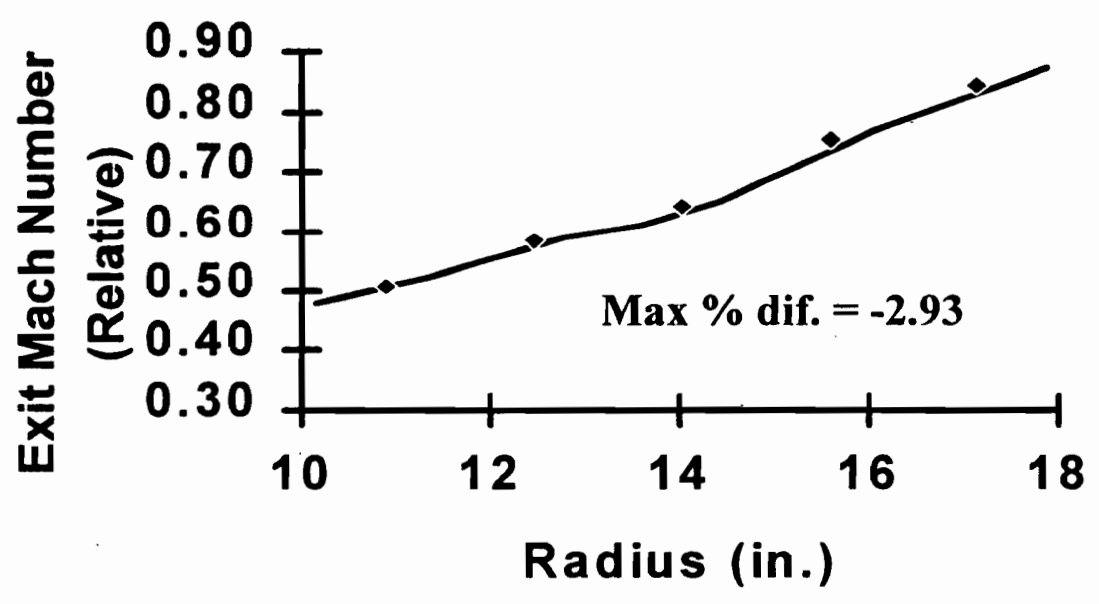

Figure E.26: Calibration, exit relative Mach number on the 100\% corrected speed line near the design throttle line. 
$\checkmark$ Data - SLCC

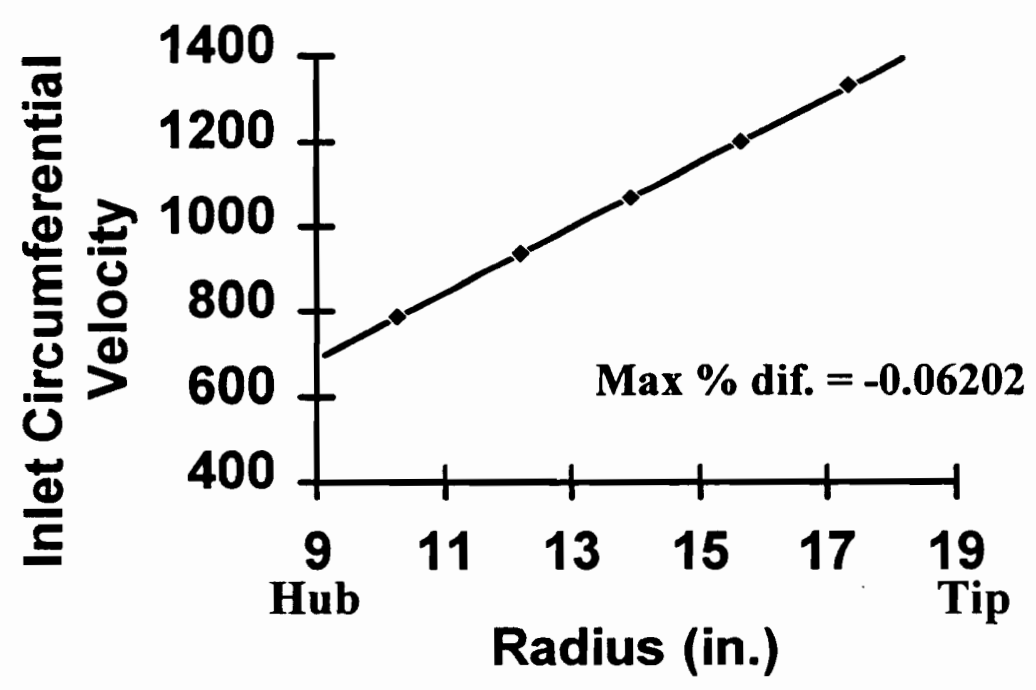

Figure E.27: Calibration, inlet relative circumferential velocity on the $100 \%$ corrected speed line near the design throttle line.

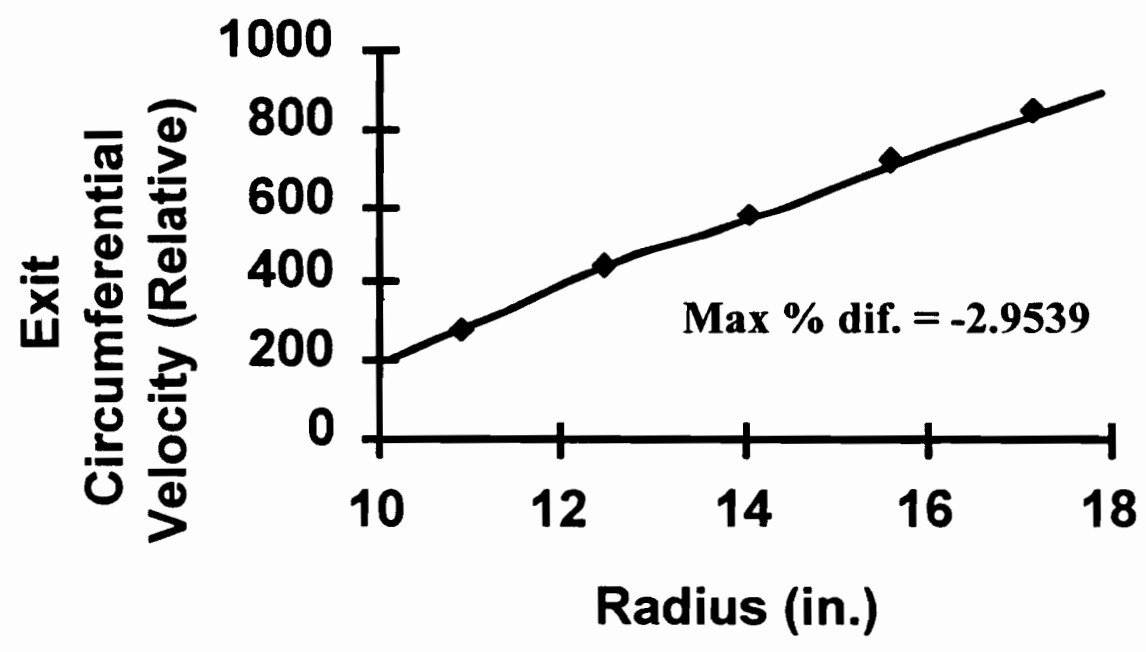

Figure E.28: Calibration, exit relative circumferential velocity on the $100 \%$ corrected speed line near the design throttle line. 


\section{Appendix F - Grid Density Investigation}

The base grid of $(69 \times 13 \times 26)$ presented earlier in Figures 6.1 and 6.2 was adequate for TEACC to use in resolving a numerical flow field. However, whether the base grid is dense enough to resolve the strong physical gradients throughout the simulation and if it was properly packed around the blade to minimize numerical inaccuracies are other issues to be resolved. These issues were investigated by doubling the grid separately in each direction (axal, radial, and circumferential), then re-solving the flow field on the denser grid and looking for changes and significant improvements to determine if the grid was dense enough in each direction. The grid was first roughly doubled in the axial direction to 131 grid points, as shown in Figure F.1. The grid was refined in this direction by identifying six regions between the physical and imposed constraints and doubling these regions. This was done so the previously defined constraints were left in the same locations before and after grid refinement. The grid doubled in the radial direction can be seen in Figure F.2 with 24 radial grid points. The only constraints in this direction are the physical inner center-body and the outer casing. The last grid doubling is in the circumferential direction, where the grid increased from 26 nodes to 50 nodes (only four segments shown in Figure F.5). Each segment is 7.5 degrees in circumferential extent.

A radial-circumferential view of the grids is presented in Figures F.3 - F.5 for easy comparison with the base grid. Only a portion of the grid is shown in this view because these grids are tailored to run in the pseudo-axisymmetric mode of NPARC, 
requiring only four circumferential segments. The grid doubled in the radial direction (Figure F.4) forms grid cells which are far from square. Although the generalized curvilinear coordinate transformation will help with these shapes, numerical accuracy problems may result. The grid doubled in the circumferential direction (Figure F.5) has the feature of nearly square grid cells. With grid construction complete, the base grid and its three refinements were compared for grid convergence.

\section{F.1 Compressor Overall Performance Improvement}

A 100-percent design corrected speed point near the throttle line was chosen as the test point to evaluate grid convergence. Since data were also available at this point a percent difference of key flow-field variables with experimental data is constructed both overall and radially. These differences checked for grid convergence and were evaluated to determine whether grid refinements improved the turbomachinery flow-field simulation. First, overall total pressure ratio and adiabatic efficiency calculated from each of the four grids are compared to experimental data and to each other in Table F.1. The base grid of $(69 \times 13 \times 26)$ gives nearly the same results as that of doubling the grids in the axial and radial directions. However, the error is cut roughly in half (from $-1.06 \%$ to $-0.41 \%$ difference in total pressure ratio and from $-2.88 \%$ to $-1.47 \%$ difference in total temperature ratio) by doubling the grid in the circumferential direction. Refinement in the circumferential direction is expected to improve the solution, since TEACC uses a Cartesian coordinate system to resolve a cylindrical turbomachinery flow field. 
An investigation of circumferential area gained by doubling the grid in the circumferential direction will help quantify the improvements obtained by doubling the circumferential grid. The truncated nature of the Cartesian grid, with the error in area clearly viewable from the cylindrical representation of the same volume, is displayed in Figure F.6. The error is a function of the angle extent in the circumferential direction. A calculation procedure was constructed to calculate area with both Cartesian and cylindrical techniques, then their ratio was constructed for comparison by the following equations:

$$
\begin{aligned}
& A_{\text {cir }}=r^{2} \frac{\theta}{2} \\
& A_{\text {tri }}=\frac{r^{2} \sin \theta}{2} \\
& \frac{A_{\text {cir }}}{A_{\text {tri }}}=\frac{\theta}{\sin \theta}
\end{aligned}
$$

A comparison of sector area to Cartesian area is presented in Table F.2. The reduction in error for an area from a 15-degree segment to a 7.5-degree segment was calculated by the ratio of twice the error obtained from the 7.5-degree segment of 0.29 percent with the error obtained from the 15-degree segment of 1.15-percent. Therefore, a 49.7-percent decrease in error results for area (from $A_{\text {cir }} / A_{\text {tri }}=1.0115$ decreasing to 1.002862 ) by doubling the number of circumferential segments.

A related exercise can be applied to the total pressure ratio and the efficiency presented in Table F.1. The percent difference of total pressure ratio and efficiency with 
experimental data for the 7.5 -degree segments are $-0.41 \%$ and $-1.47 \%$, respectively. The averages of the percent difference of total pressure ratio and efficiency with experimental data of the other three grids all with 15-degree segments are $-1.06 \%$ and $-2.88 \%$, respectively. The reductions in error for total pressure ratio and efficiency are calculated from one minus the ratio of the percent differences of the 7.5-degree segment to the percent differences of the 15-degree segment. Therefore, the reduction in error for total pressure due to doubling the grid in the circumferential direction is $60.9 \%$, and the reduction in error for efficiency is $48.9 \%$. Recall the reduction in error for area going to the finer circumferential grid was $49.7 \%$. Therefore, the reduction in error for efficiency is completely accounted for by volume calculations due to a Cartesian representation of the geometry. Certainly, most of the reduction in total pressure ratio is also accounted for with the greater accuracy in volume calculations associated with the 7.5-degree segments.

\section{F.2 Compressor Radial Performance Improvement}

Grid convergence between the four grids was also evaluated in a radial sense for six flow variables as a representative set to adequately describe the flow field at the same $100 \%$ speed point used for the above overall analysis. A radial percent difference of each variable was constructed with experimental data for each grid and plotted together for comparison in Figures F.7 - F.12. The variables used to construct the percent differences for comparisons were total pressure ratio, total temperature ratio, exit absolute velocity, exit absolute circumferential velocity, exit static pressure, and exit static temperature. Some overall observations can be made concerning each figure. First, the results 
produced on each grid are nearly the same, indicating that doubling the grid in any one direction does not significantly improve or change the flow field from the base grid. Second, the variables of the flow field compare within 4 percent of the experimental data except for exit static temperature (within 6 percent) and exit absolute circumferential velocity (within 8 percent).

\section{F.3 Summary of Grid Study}

The flow field produced by doubling the grid in the circumferential direction does not reveal a noticeable radial improvement in the flow field compared to the flow fields produced by the base grid. This is a further indication that the improvement in overall pressure ratio and efficiency observed earlier was a direct result of accounting for more volume or more mass flow due to the finer Cartesian grid construction. The base grid was selected for all subsequent calculations since the improvements offered by the grid refinements were not significant enough to give up the faster wall clock time to solution. 
Table F.1: Grid refinement investigation at $100 \%$ corrected speed near design throttle line.

\begin{tabular}{|c|c|c|c|c|}
\hline \multicolumn{3}{|c|}{ Overall Performance } & \multicolumn{2}{c|}{ Percent Difference } \\
\hline Data or Grids & $\begin{array}{c}\text { Total Pressure } \\
\text { Ratio }\end{array}$ & Efficiency & $\begin{array}{c}\text { Total Pressure } \\
\text { Ratio }\end{array}$ & Efficiency \\
\hline Data & 1.6870 & 0.8972 & & \\
\hline $69 \times 13 \times 26$ & 1.6718 & 0.8742 & -0.9010 & -2.5635 \\
\hline $131 \times 13 \times 26$ & 1.6677 & 0.8693 & -1.1440 & -3.1097 \\
\hline $69 \times 24 \times 26$ & 1.6677 & 0.8706 & -1.1440 & -2.9659 \\
\hline $69 \times 13 \times 50$ & 1.6800 & 0.8840 & -0.4149 & -1.4712 \\
\hline
\end{tabular}

Table F.2: Circumferential area relationship between cylindrical area and Cartesian area.

\begin{tabular}{|l|c|c|}
\hline Area Relations Between Cartesian and Cylindrical Coordinates & A & B \\
\hline Angle Between Circumferential Segments (degrees) & 15 & 7.5 \\
\hline Number of Segments Within Circumferential Extent & 24 & 48 \\
\hline Area (circle) / Area (triangle) & 1.0115 & 1.0029 \\
\hline Percent Error of Area (circle) to Area (triangle) & 1.1515 & 0.2862 \\
\hline
\end{tabular}




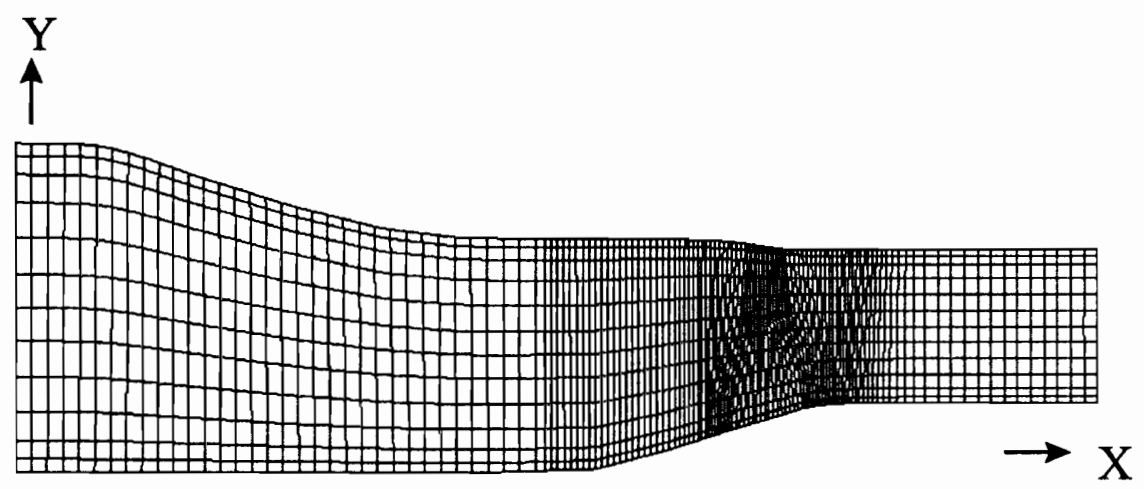

Figure F.1: Axial-radial view of the grid doubled in the axial direction from the base grid with grid nodes $(131 \times 13 \times 26)$.

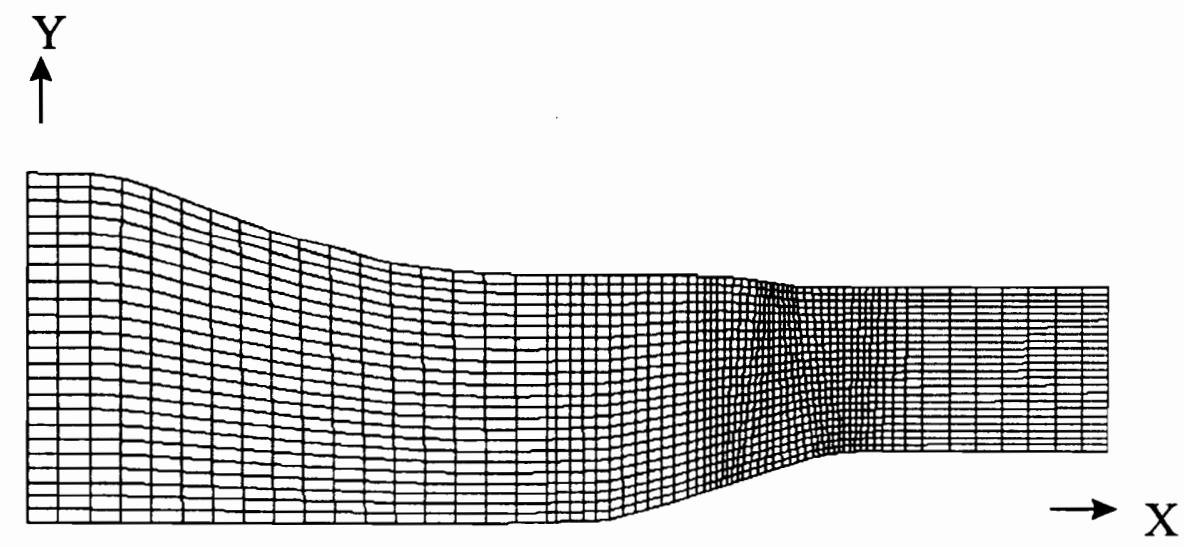

Figure F.2: Axial-radial view of the grid doubled in the radial direction from the base grid with nodes $(69 \times 24 \times 26)$. 


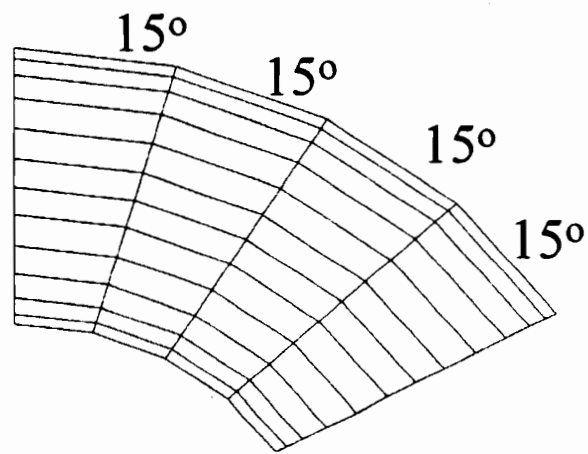

Figure F.3: Radial-circumferential view of the three-dimensional base grid $(69 \times 13 \times 26)$.

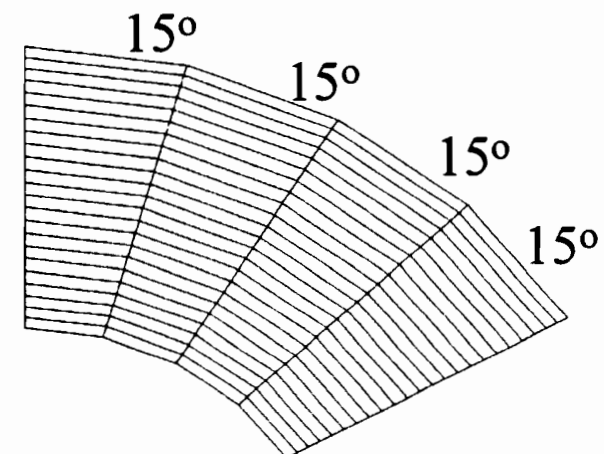

Figure F.4: Radial-circumferential view of the grid doubled in the radial direction from the base grid with nodes $(69 \times 24 \times 26)$.

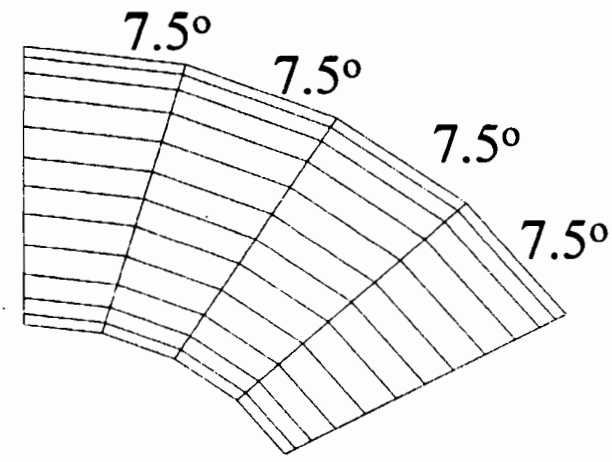

Figure F.5: Radial-circumferential view of the grid doubled in the circumferential direction from the base grid with nodes $(69 \times 13 \times 50)$. 

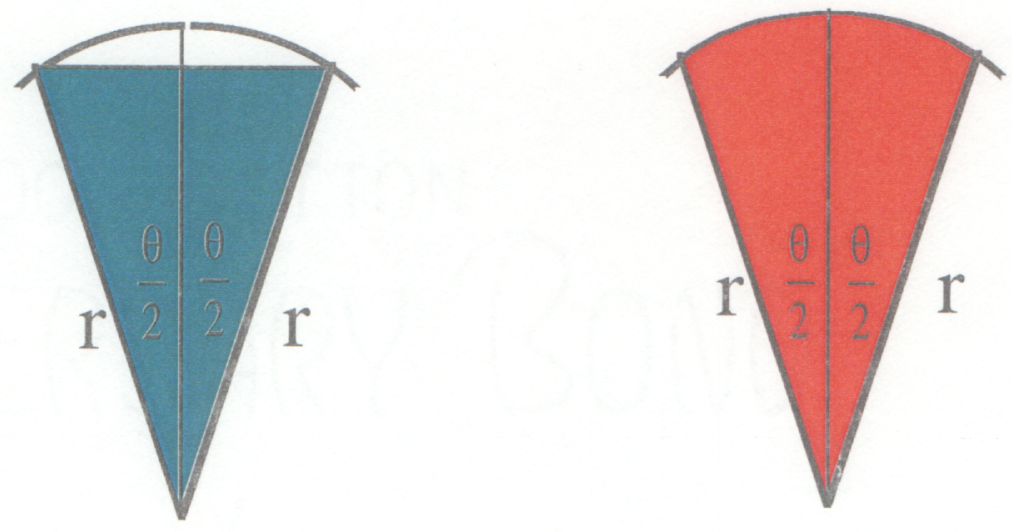

$$
\mathrm{A}_{\text {Triangle }}=\frac{\mathrm{r}^{2} \sin \theta}{2}
$$$$
\mathrm{A}_{\text {Sector }}=\mathrm{r}^{2} \frac{\theta}{2}
$$

$$
A_{\text {Correction }}=\frac{A_{\text {Sector }}}{A_{\text {Triangle }}}=\frac{\theta}{\sin \theta}
$$

Figure F.6: Area correction for converting from Cartesian control volumes. 


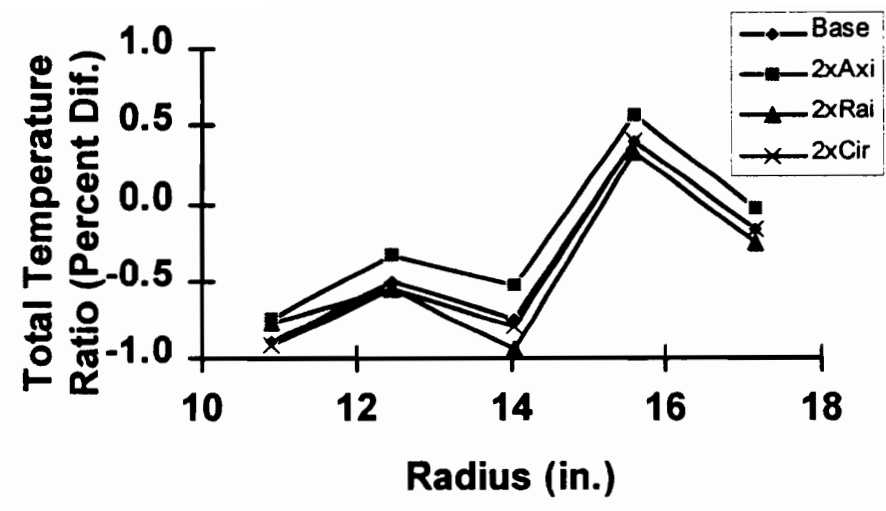

Figure F.7: Percent difference of total temperature ratio with experimental data for the base grid and the grid doubled in the axial, radial, and circumferential direction.

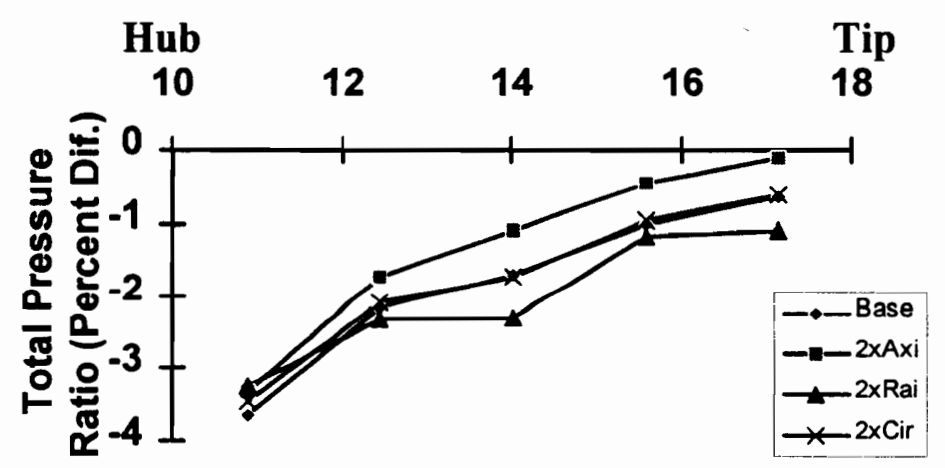

Radius (in.)

Figure F.8: Percent difference of total pressure ratio with experimental data for the base grid and the grid doubled in the axial, radial, and circumferential direction. 


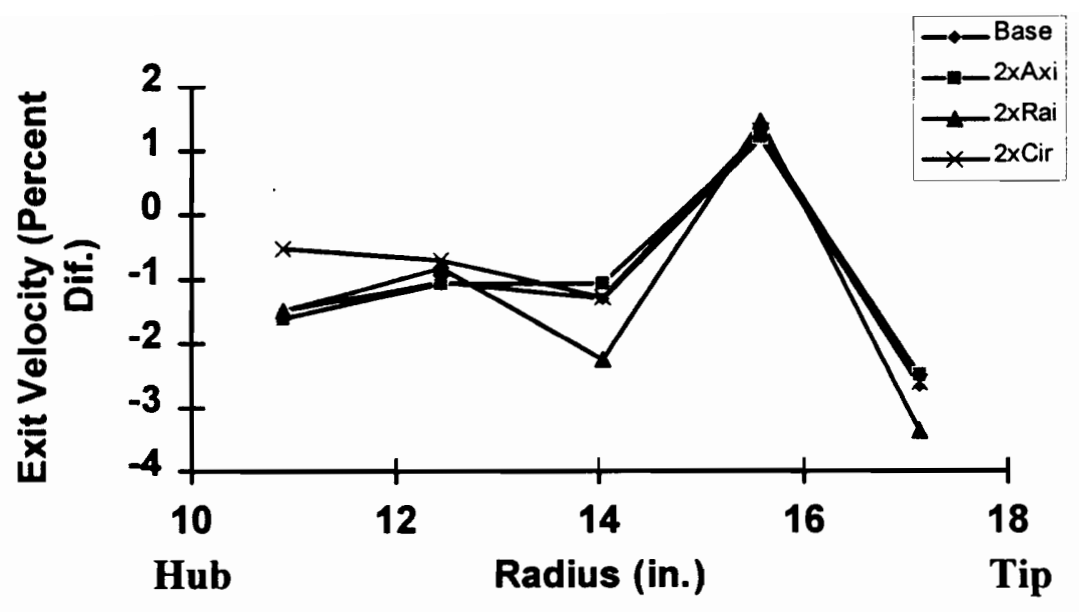

Figure F.9: Percent difference of exit absolute velocity with experimental data for the base grid and the grid doubled in the axial, radial, and circumferential direction.

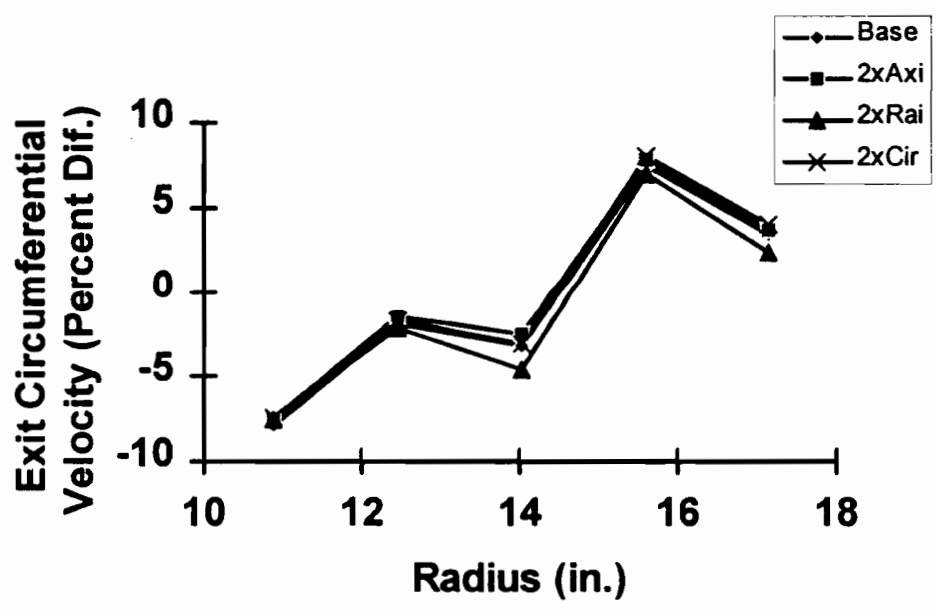

Figure F.10: Percent difference of exit circumferential velocity with experimental data for the base grid and the grid doubled in the axial, radial, and circumferential direction. 


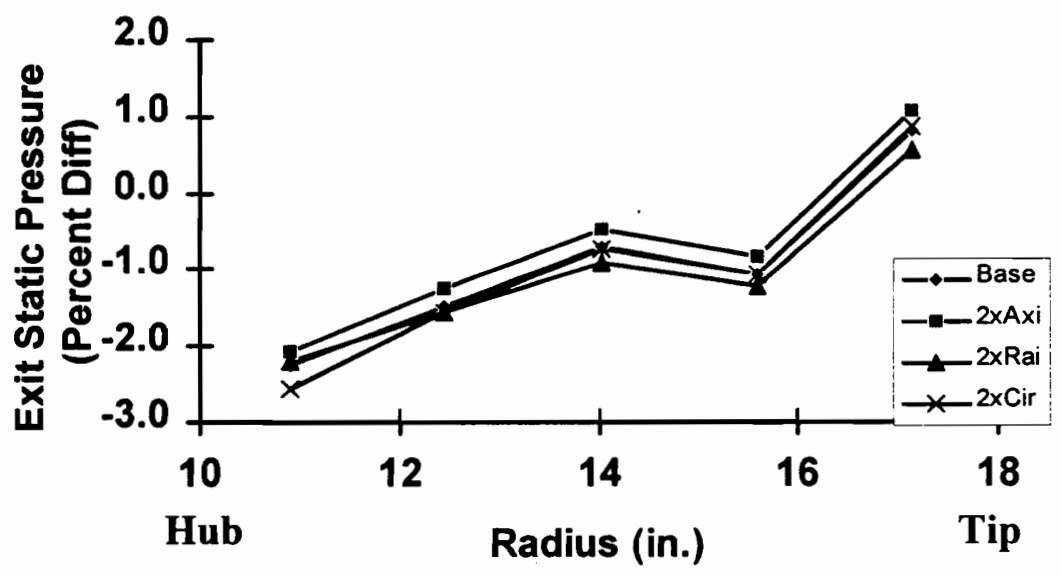

Figure F.11: Percent difference of exit static pressure with experimental data for the base grid and the grid doubled in the axial, radial, and circumferential direction.

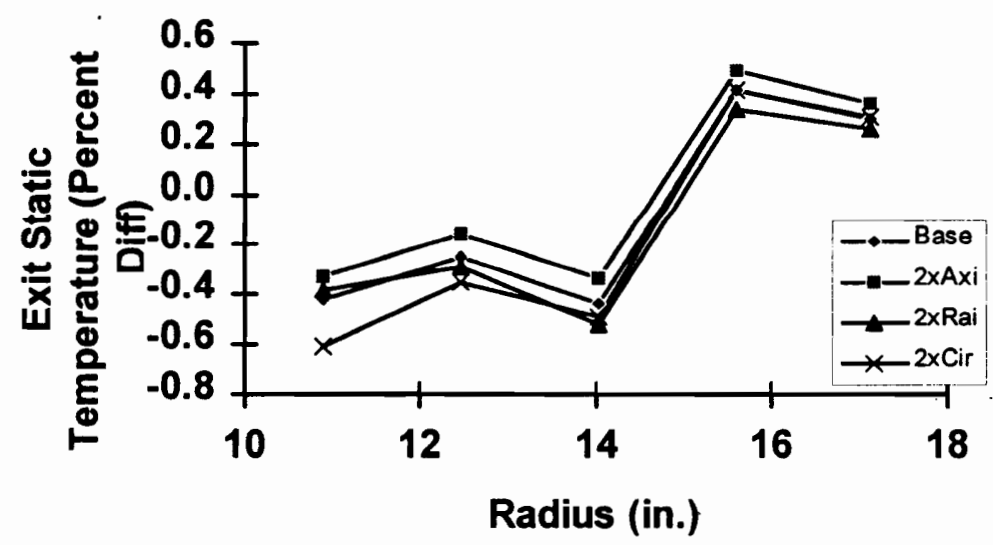

Figure F.12: Percent difference of exit static temperature with experimental data for the base grid and the grid doubled in the axial, radial, and circumferential direction. 


\section{Vita}

Alan Akers Hale was born in Roanoke, Virginia, in June 1960 to Mr. Ralph Mitchell Hale and Mrs. Ruby Akers Hale. He grew up on a small farm in the beautiful Shenandoah Valley with his parents and older brother, Lance Mitchell Hale. He attended East Vinton Elementary and William Byrd Intermediate Schools in Vinton, Virginia, and graduated from William Byrd High School in June 1978. Mr. Hale graduated from Virginia Military Institute with a Bachelor of Science degree in Civil Engineering, and was commissioned a Second Lieutenant in the United States Army in May 1982. The following summer, he continued his education at Virginia Polytechnic Institute and graduated with a Master of Science degree in Mechanical Engineering in June 1984. Upon graduation, he accepted employment with Sverdrup Technology, Inc. at the Arnold Engineering Development Center in Tullahoma, Tennessee, and married Julie Kay Scheiman. He continued his course work at the University of Tennessee Space Institute while working full time with Sverdrup Technology, Inc. In August 1993, he took a leave of absence from Sverdrup Technology, Inc., and he and his family moved to Blacksburg, Virginia, for one year to complete the residency requirements and course work at Virginia Polytechnic Institute toward a Doctor of Philosophy degree. Mr. Hale and family returned to Tullahoma in August 1994, where he completed his research.

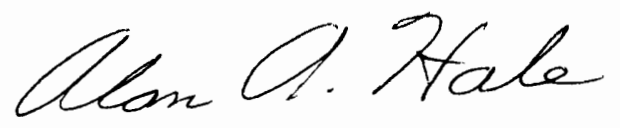

